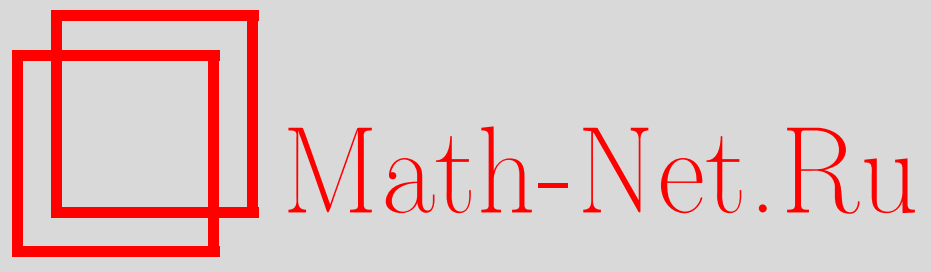

М. И. Вишик, В. В. Чепыжов, Траекторные аттракторы уравнений математической физики, УМH, 2011, том 66, выпуск 4, 3-102

DOI: https://doi.org/10.4213/rm9407

Использование Общероссийского математического портала Math-Net.Ru подразумевает, что вы прочитали и согласны с пользовательским соглашением http://www . mathnet.ru/rus/agreement

Параметры загрузки:

IP: 54.197 .217 .227

26 апреля 2023 г., 14:01:33

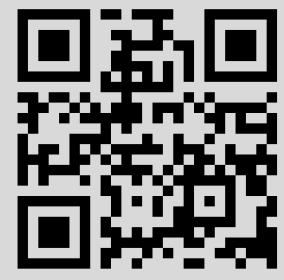


УДК 517.958

\title{
Траекторные аттракторы уравнений математической физики
}

\author{
М. И. Вишик, В. В. Чепыжов
}

\begin{abstract}
В данном обзоре излагается метод траекторных динамических систем и траекторных аттракторов, который применяется к исследованию предельного асимптотического поведения решений нелинейных эволюционных уравнений. Этот метод особенно полезен при изучении диссипативных уравнений математической физики, для которых соответствующая начальная задача Коши имеет глобальное (слабое) решение по времени, но единственность этого решения или не установлена, или не имеет места. Важным примером такого уравнения служит 3D-система Навье-Стокса в ограниченной области. В такой ситуации нельзя напрямую воспользоваться классической схемой построения динамической системы в фазовом пространстве начальных условий задачи Коши данного уравнения и найти глобальный аттрактор этой динамической системы. Тем не менее, для таких уравнений можно построить траекторную динамическую систему и исследовать траекторный аттрактор соответствующей трансляционной полугруппы. Этот универсальный метод применяется для разнообразных типов уравнений, возникающих в математической физике: для общих диссипативных систем реакции-диффузии, для 3D-системы Навье-Стокса, для диссипативных волновых уравнений, для нелинейных эллиптических уравнений в цилиндрических областях и для других уравнений и систем. Отдельное внимание уделяется использованию метода траекторных аттракторов в задачах приближения и возмущения, возникающих в сложных моделях математической физики.
\end{abstract}

Библиография: 96 названий.

Ключевые слова: динамические системы, траекторные аттракторы, уравнения математической физики, некорректные задачи.

\section{СоДЕРЖАНИЕ}

Глава I. Глобальные аттракторы эволюционных уравнений ........... 10

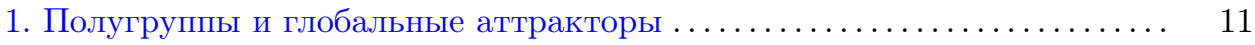

2. Глобальные аттракторы уравнений математической физики ......... 14

Глава II. Траекторные аттракторы уравнений математической физики .. 24

1. Траекторный аттрактор для модельной системы ОДУ $\ldots \ldots \ldots \ldots \ldots \ldots .25$

2. Траекторный аттрактор общей системы реакции-диффузии ......... 33

(C) М. И. Вишик, В. В. Чепыжов, 2011 
3. Двумерная система Эйлера с диссипацией и ее траекторный аттрактор

4. Общая схема построения траекторного аттрактора эволюционного уравнения . ............................................ 50

5. Траекторный аттрактор трехмерной системы Навье-Стокса .......... 58

6. Траекторный аттрактор нелинейного волнового уравнения ......... 66

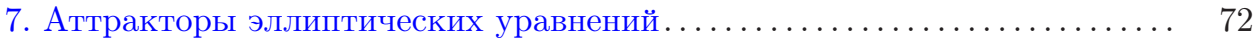

Глава III. Некоторые вопросы аппроксимации и возмущения траекторных

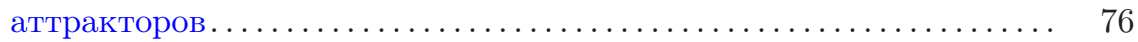

1. 3D-система Навье-Стокса: приближение траекторными аттракторами

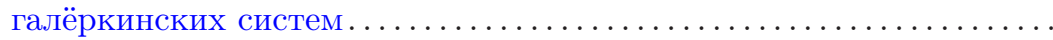

2. 3D-система Навье-Стокса: аппроксимация траекторными аттракторами

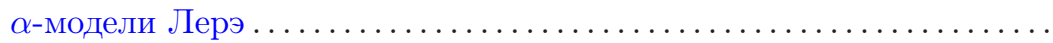

3. Предел нулевой вязкости траекторного аттрактора диссипативной 2Dсистемы Навье-Стокса........................... 89

4. Система реакции-диффузии с малым коэффициентом диффузии ...... 92

4.1. Слабые решения системы реакции-диффузии ............... 92

Список литературы . . . . . . . . . . . . . . . . . . . . . . . . 97

\section{Введение}

Многие актуальные проблемы, возникающие в различных областях физики и механики, связаны с изучением сложных математических моделей, которые описываются эволюционными уравнениями с частными производными. Задача прогнозирования в таких моделях сводится к изучению решений этих эволюционных уравнений на больших интервалах времени, а также в пределе, когда время стремится к бесконечности. С этой проблематикой тесно связаны вопросы об устойчивости и неустойчивости предельных структур, которые выделяются из всего многообразия возможных состояний модели.

В последние годы существенный прогресс при решении подобных задач достигнут при систематическом применении теории аттракторов бесконечномерных динамических систем. Бесконечномерные динамические системы, в частности, порождаются эволюционными уравнениями с частными производными, а аттракторы позволяют выделить самые существенные предельные множества решений, которые характеризуют всю динамику сложной модели.

Для многих важных эволюционных уравнений математической физики было показано, что долговременное поведение их решений можно описывать с помощью конечномерных глобальных аттракторов (см. монографии [1]-[6] и цитируемую в них обширную литературу).

Общую динамическую систему принято задавать в виде пары $(\mathscr{E},\{\mathscr{S}(t)\})$, которая состоит из фазового пространства $\mathscr{E}$ и действующей на нем полугруппы операторов $\mathscr{S}(t): \mathscr{E} \rightarrow \mathscr{E}$, зависящих от вещественной переменной $t \geqslant 0$, которая называется временем. Обычно предполагается, что фазовое пространство $\mathscr{E}$ является полным метрическим или банаховым пространством,

Работа выполнена при поддержке РФФИ (гранты № 11-01-00339, 10-01-00293). 
которое может иметь конечную или бесконечную размерность. Операторы $\{\mathscr{S}(t), t \geqslant 0\}$ образуют полугруппу, т. е. выполнены соотношения

$$
\mathscr{S}(0)=\mathrm{Id}, \quad \mathscr{S}\left(t_{1}\right) \circ \mathscr{S}\left(t_{2}\right)=\mathscr{S}\left(t_{1}+t_{2}\right), \quad \forall t_{1}, t_{2} \geqslant 0 .
$$

Здесь и далее Id обозначает тождественный оператор.

В приложениях к эволюционным уравнениям вида

$$
\partial_{t} u=-F(u),
$$

где $F(u)$ - некоторый нелинейный дифференциальный оператор, динамическую систему можно построить следующим образом. Дополним уравнение (0.1) начальным условием

$$
\left.u\right|_{t=0}=u_{0}, \quad u_{0} \in E,
$$

где $u_{0}$ - произвольный элемент из некоторого пространства $E$. Предположим, что при любом $u_{0} \in E$ задача Коши $(0.1),(0.2)$ имеет единственное решение $u(t), t \geqslant 0$, причем $u(t) \in E$ при всех $t \geqslant 0$. При выполнении этого существенного предположения однозначно определены операторы сдвига вдоль решений уравнения (0.1):

$$
S(t) u_{0}:=u(t), \quad t \geqslant 0,
$$

где $u(t), t \geqslant 0,-$ решение задачи $(0.1),(0.2)$ с начальным условием $u_{0}$, стоящим в левой части равенства (0.3). Легко видеть, что пара $(E,\{S(t)\})$ образует динамическую систему.

Как уже отмечалось, для описания общей динамической системы $(\mathscr{E},\{\mathscr{S}(t)\})$ применяется понятие глобального аттрактора.

ОПРеДЕЛЕНИЕ 0.1. Множество $\mathscr{A} \subset \mathscr{E}$ называется глобалъным аттрактором полугруппы $\{\mathscr{S}(t)\}$, если оно обладает следующими свойствами:

1) $\mathscr{A}$ является компактным множеством в топологии пространства $\mathscr{E}$;

2) $\mathscr{A}$ притягивает в топологии $\mathscr{E}$ при $t \rightarrow \infty$ сдвиги $\mathscr{S}(t) B$ любого ограниченного множества $B$ из $\mathscr{E}$;

$3)$ множество $\mathscr{A}$ строго инвариантно относительно полугруппы $\{\mathscr{S}(t)\}$, т. е.

$$
\mathscr{S}(t) \mathscr{A}=\mathscr{A} \quad \text { для любого } t \geqslant 0 .
$$

Отметим, что глобальные аттракторы можно строить не для любых динамических систем, но лишь для тех, которые являются диссипативными. На физическом языке диссипативность означает существенную потерю энергии при эволюции по времени у начальных состояний системы, энергия которых была велика. На математическом языке функционал энергии часто трактуется как норма состояния в соответствующем фазовом пространстве $\mathscr{E}$, поэтому диссипативность - это существование некоторого, возможно, большого, но ограниченного по норме в $E$ поглощающего множества полугруппы $\{\mathscr{S}(t)\}$, например, шара $B_{\rho}$ в пространстве $\mathscr{E}$ с радиусом $\rho>0$, обладающего тем свойством, что если множество $B \subset \mathscr{E}$ ограничено, то $\mathscr{S}(t) B \subseteq B_{\rho}$ при всех $t \geqslant \tau(B)$, где момент времени $\tau$ зависит от $B$.

В главе I настоящей статьи, имеющей предварительный характер, сформулированы классические теоремы о существовании и структуре глобальных 
аттракторов общих диссипативных полугрупп, действующих в полных метрических или банаховых пространствах. Кроме того, в этой главе приведены примеры типичных диссипативных уравнений и систем математической физики, для которых справедлива теорема единственности решения соответствующей задачи Коши. Эти уравнения порождают динамические системы по описанной выше схеме, и для них удается построить глобальные аттракторы в соответствующем пространстве $E$ начальных условий, а также исследовать свойства аттракторов. Рассмотрены следующие уравнения: система уравнений реакции-диффузии, двумерная система уравнений Навье-Стокса, волновое уравнение с диссипацией и комплексное уравнение Гинзбурга-Ландау. Каждое из перечисленных уравнений играет важную роль в соответствующем разделе математической физики. Поэтому глобальные аттракторы для этих уравнений весьма интенсивно изучались и продолжают изучаться в математической литературе, ссылки на которую приводятся в главе I по ходу изложения.

Несмотря на чрезвычайную важность и плодотворность классического метода построения динамической системы, который основан на свойстве единственности решения задачи Коши вида $(0.1),(0.2)$, приходится констатировать, что существует значительный класс “некорректных" уравнений математической физики, для которых свойство единственности или не выполнено, или не доказано, вопреки значительным усилиям многих математиков решить эти проблемы. Наиболее известным примером такого рода является трехмерная система Навье-Стокса, для которой существование глобальных слабых решений было доказано в классических работах Лерэ и Хопфа, но установить единственность задачи Коши для них пока не удается. С другой стороны, для этого уравнения известны разные классы сильных решений, в которых единственность имеет место, но в этих классах удается строить лишь локальные по времени решения. Аналогичная ситуация наблюдается и для других важных уравнений математической физики. Для изучения эволюции решений таких уравнений приходится применять иные подходы, одним из которых является метод траекторных аттракторов (см. [7]-[12], аналогичный подход использовался в [13]).

Опишем кратко схему построения траекторного аттрактора для общего эволюционного уравнения вида (0.1), следуя в основном работе [12]. Подробное изложение составляет содержание главы II. Переменную времени мы будем иногда обозначать буквой $s$. В этом случае она становится внутренней переменной некоторого функционального пространства. Предполагается, что для уравнения (0.1) выделено некоторое семейство его решений $\{u(s), s \geqslant 0\}$, которые определены на всей полуоси времени $\mathbb{R}_{+}=\{0 \leqslant s<+\infty\}$. Такие (глобальные) решения называются траекториями уравнения (0.1), а выделенное семейство траекторий называется пространством траекторий и обозначается $\mathscr{K}^{+}$. В конкретных приложениях множество $\mathscr{K}^{+}$состоит из сильных или слабых решений рассматриваемого уравнения. Кроме того, часто приходится накладывать дополнительные априорные ограничения, продиктованные свойствами диссипативности изучаемой модели.

Предполагается, что пространство траекторий $\mathscr{K}^{+}$принадлежит некоторому линейному функциональному пространству $\mathscr{F}_{+}^{\text {loc }}=\{f(s), s \geqslant 0\}$. Про- 
странство $\mathscr{F}_{+}^{\text {loc }}$ состоит из функций $f(s), s \geqslant 0$, со значениями в некотором банаховом пространстве $E$. При этом функции $f(t)$ из $\mathscr{F}_{+}^{\text {loc }}$, рассматриваемые на интервале $\{0 \leqslant t \leqslant M\}$ при каждом $M>0$, принадлежат некоторому пространству $\mathscr{F}(0, M ; E)$, которое является банаховым. Например, $\mathscr{F}(0, M ; E)=C([0, M] ; E)$ - пространство непрерывных функций со значениями в $E$. Другие примеры пространств $\mathscr{F}(0, M ; E)$ приведены в главе II.

Рассмотрим трансляционные операторы $T(h), h \geqslant 0$, которые действуют в пространстве $\mathscr{F}_{+}^{\text {loc }}$ по формуле

$$
T(h) f(s)=f(h+s), \quad \forall h \geqslant 0 .
$$

Предположим, что трансляции переводят пространство $\mathscr{F}+{ }_{+}$в себя. Тогда, очевидно, операторы $\{T(h), h \geqslant 0\}=:\{T(h)\}$ образуют полугруппу:

$$
T(0)=\mathrm{Id}, \quad T\left(h_{1}\right) T\left(h_{2}\right)=T\left(h_{1}+h_{2}\right), \quad \forall h_{1}, h_{2} \geqslant 0 .
$$

Заменим переменную $h$ на переменную времени $t$, которая фигурирует в определении динамической системы, и рассмотрим действие трансляционной полугруппы $\{T(t)\}$ на $\mathscr{K}^{+}$.

Предполагается, что пространство траекторий $\mathscr{K}^{+}$является трансляционно инвариантным. Это означает, что функция $u(s+h)$ принадлежит $\mathscr{K}^{+}$при любом $h \geqslant 0$, если известно, что $u \in \mathscr{K}^{+}$. Такое свойство всегда выполнено при естественном задании пространства решений $\mathscr{K}^{+}$, так как уравнение $(0.1)$ является автономным. Следовательно, трансляционная полугруппа $\{T(t)\}$ отображает $\mathscr{K}^{+}$в себя:

$$
T(t) \mathscr{K}^{+} \subseteq \mathscr{K}^{+}, \quad \forall t \geqslant 0 .
$$

Мы построили новую динамическую систему $\left(\mathscr{K}^{+},\{T(t)\}\right)$ в которой фазовым пространством является пространство траекторий $\mathscr{K}^{+}$, а полугруппой на $\mathscr{K}^{+}$служит трансляционная полугруппа $\{T(t)\}$. Задача заключается в изучении глобального аттрактора $\mathfrak{A}$ построенной нами траекторной динамической системы. Этот глобальный аттрактор называется траекторным аттрактором полугруппы $\{T(t)\}$ на $\mathscr{K}^{+}$.

Для построения аттрактора необходимо иметь топологию в фазовом пространстве $\mathscr{K}^{+}$. В конкретных приложениях эта топология не всегда задается метрикой или нормой метрического или банахова пространства. Часто приходится обращаться к более слабым топологиям. Приходиться также быть осторожным при определении "ограниченного" семейства траекторий в пространстве $\mathscr{K}^{+}$. Используемая при этом метрика часто бывает не связана с топологией, в которой рассматривается притяжение к аттрактору. (Здесь мы используем понятие $(F, D)$-аттрактора полугруппы из монографии [1].)

Пусть в пространствах $\mathscr{F}(0, M ; E)$ задана некоторая топология, которая обозначается $\Theta(0 ; M ; E)$. Для простоты предполагается, что топология $\Theta(0 ; M ; E)$ является хаусдорфовой и полностью задается через сходимость последовательностей. Обычно в приложениях $\Theta(0, M ; E)$ является топологией сильной или слабой сходимости на отрезке времени $[0, M]$. Например, если $\mathscr{F}(0, M ; E)=$ $C([0, M] ; E)$, то топология $\Theta(0, M ; E)$ - это топология равномерной сходимости на отрезке $[0, M]$ по норме $E$. 
В пространстве $\mathscr{F}_{+}^{\text {loc }}$ определяется топология $\Theta_{+}^{\text {loc }}$, которая порождается локальной сходимостью в $\Theta(0, M ; E)$ при каждом $M>0$. Точнее, по определению, последовательность $\left\{f_{m}(\cdot), m \in \mathbb{N}\right\}$ из $\mathscr{F}_{+}^{\text {loc }}$ сходится к элементу $f(\cdot) \in \mathscr{F}_{+}^{\text {loc }}$ в топологии $\Theta_{+}^{\text {loc }}$ при $m \rightarrow \infty$, если при любом $M>0$ последовательность $\left\{f_{m}(\cdot)\right\}$ из $\mathscr{F}(0, M ; E)$ сходится к $f(\cdot) \in \mathscr{F}(0, M ; E)$ в топологии $\Theta(0, M ; E)$ при $m \rightarrow \infty$. Топология $\Theta_{+}^{\text {loc } я в л я е т с я ~ х а у с д о р ф о в о и ̆ . ~ К р о м е ~ т о г о, ~}$ она метризуема, если исходные локальные топологии $\Theta(0, M ; E)$ были метризуемыми. (Топологию $\Theta_{+}^{\text {loc }}$ иногда называют индуктивным пределом топологий $\Theta(0, M ; E)$ при $M \rightarrow \infty$.)

Легко доказать, что полугруппа трансляций $\{T(t)\}$ непрерывна в топологии $\Theta_{+}^{\text {loc }}$ индуктивного предела. Это обстоятельство сильно облегчает построение и исследование траекторных аттракторов.

Определяется подпространство $\mathscr{F}_{+}^{\mathrm{b}} \subset \mathscr{F}_{+}^{\text {loc }}$ функций $f \in \mathscr{F}_{+}^{\text {loc }}$, обладающих конечной нормой

$$
\|f\|_{\mathscr{F}+}:=\sup _{h \geqslant 0}\left\|\Pi_{0,1} f(s+h)\right\|_{\mathscr{F}(0,1 ; E)} .
$$

Норма пространства $\mathscr{F}_{+}^{\mathrm{b}}$ необходима для задания класса ограниченных множеств в пространстве траекторий $\mathscr{K}^{+}$. Предполагается, что

$$
\mathscr{K}^{+} \subset \mathscr{F}_{+}^{\mathrm{b}}
$$

ОПРЕДЕЛЕНИЕ 0.2. Множество $\mathfrak{A} \subseteq \mathscr{K}^{+}$называется траекторным атmрактором полугруппы $\{T(t)\}$ на $\mathscr{K}^{+}$в топологии $\Theta_{+}^{\text {loc }}$, если:

1) множество $\mathfrak{A}$ ограничено в $\mathscr{F}_{+}^{\mathrm{b}}$ и компактно в топологии $\Theta_{+}^{\text {loc }}$;

2) $\mathfrak{A}$ является притягивающим множеством, т. е. для любого ограниченного в $\mathscr{F}_{+}^{\mathrm{b}}$ множества $\mathfrak{B} \subset \mathscr{K}^{+}$и для любой окрестности $\mathscr{O}(\mathfrak{A})$ в $\Theta_{+}^{\text {loc }}$ найдется число $\tau=\tau(\mathfrak{B}, \mathscr{O}) \geqslant 0$ такое, что $T(t) \mathfrak{B} \subseteq \mathscr{O}(\mathfrak{A})$ при всех $t \geqslant \tau$;

3) $\mathfrak{A}$ строго инвариантно, т. е. $T(t) \mathfrak{A}=\mathfrak{A}$ для всех $t \geqslant 0$.

Для построения траекторных аттракторов уравнений вида (0.1) используется следующая основная теорема.

Теорема 0.1. Пусть в пространстве траекторий $\mathscr{K}^{+}$уравнения (0.1) удалось построить множество $\mathscr{P} \subseteq \mathscr{K}^{+}$, ограниченное в $\mathscr{F}_{+}^{\mathrm{b}}$ и компактное в $\Theta_{+}^{\text {loc }}$, которое является поглощающим для полугруппы трансляиий $\{T(t)\}$, m.е. для любого ограниченного в $\mathscr{F}+$ множества $\mathfrak{B} \subset \mathscr{K}^{+}$множество $T(t) \mathfrak{B}$ принадлежит $\mathscr{P}$ при всех $t \geqslant \tau(\mathfrak{B})$. Тогда трансляиионная полугруппа $\{T(t)\}$ имеет траекторный аттрактор $\mathfrak{A} \subseteq \mathscr{P}$.

Обычно (в приложениях) компактным поглощающим множеством служит множество $\mathscr{P}=\mathscr{K}^{+} \cap B_{R}$, где $B_{R}=\left\{u \mid\|u\|_{\mathscr{F}_{+}^{\mathrm{b}}} \leqslant R\right\}-$ шар в $\mathscr{F}_{+}^{\mathrm{b}}$ достаточно большого радиуса. При этом свойство поглощения обычно следует из неравенства вида

$$
\|T(t) u\|_{\mathscr{F}_{+}^{\mathrm{b}}} \leqslant C\left(\|u\|_{\mathscr{F}_{+}^{\mathrm{b}}}\right) \exp (-\gamma t)+R_{0}, \quad \forall t \geqslant 0 \quad(\gamma>0) .
$$

Как правило, в конкретных приложениях неравенство (0.4) доказывается с помощью основных априорных оценок изучаемой задачи. 
Поясним характер притяжения любого ограниченного в $\mathscr{F}_{+}^{\mathrm{b}}$ множества траекторий $\mathfrak{B}$ из $\mathscr{K}^{+}$под действием трансляций $T(t)$ к траекторному аттрактору $\mathfrak{A}$. Пусть выполнены условия основной теоремы 0.1. Тогда при любом $M>0$ множество $T(t) \mathfrak{B}$ стремится к $\mathfrak{A}$ в топологии $\Theta(0, M ; E)$ при $t \rightarrow+\infty$. Например, если топология $\Theta(0, M ; E)$ метризуема, то

$$
\operatorname{dist}_{\Theta(0, M ; E)}\left(\Pi_{0, M} T(t) \mathfrak{B}, \Pi_{0, M} \mathscr{K}\right) \rightarrow 0 \quad(t \rightarrow+\infty),
$$

где $\operatorname{dist}_{X}(\cdot, \cdot)$ обозначает отклонение по Хаусдорфу множеств в метрике пространства $X$.

Описанная схема построения траекторного аттрактора может показаться достаточно абстрактной. По этой причине глава II начинается с рассмотрения в качестве уравнения (0.1) системы обыкновенных дифференциальных уравнений в евклидовом пространстве $\mathbb{R}^{N}$. Для этого модельного уравнения проделаны все шаги построения траекторной динамической системы и траекторного аттрактора, которые имеют несложные обоснования.

В последующих разделах главы II построены траекторные аттракторы для таких важных уравнений математической физики как общая диссипативная система реакции-диффузии, двумерная система Эйлера с диссипацией, трехмерная система Навье-Стокса, диссипативные волновые уравнения и, наконец, нелинейные эллиптические уравнения в цилиндрических областях.

Еще раз подчеркнем, что для всех этих уравнений теорема единственности решения задачи Коши не доказана или не имеет места. В наших примерах траекторные аттракторы строятся прежде всего в слабых топологиях $\Theta_{+}^{\text {loc }}=\Theta_{+}^{\mathrm{w}, \text { loc }}$, отвечающих изучаемым задачам (например, для 3D-системы Навье-Стокса). Однако для некоторых из этих уравнений (например, для системы реакции-диффузии или для эллиптической системы) удается доказать притяжение к построенному траекторному аттрактору и его компактность также и в соответствующей сильной топологии $\Theta_{+}^{\mathrm{s}, \mathrm{loc}}$.

Мы начали с понятия глобального аттрактора полугруппы $\{S(t)\}$, действующей в пространстве $E$, которая отвечает уравнению вида (0.1) при условии, что задача Коши $(0.1)$, (0.2) однозначно разрешима. Если же свойство единственности не установлено, то для уравнения (0.1) во многих случаях можно найти траекторный аттрактор. В главе II показано, как, имея траекторный аттрактор, можно построить обобщенный глобальный аттрактор уравнения (0.1) в пространстве $E$ без свойства единственности задачи Коши.

Отметим, что при выполнении свойства единственности решения задачи Коши обобщенный глобальный аттрактор совпадает с обычным глобальным аттрактором.

В некоторых приложениях (например, для 3D-системы Навье-Стокса), обобщенный глобальный аттрактор удается построить лишь в слабой топологии, поэтому он называется слабым глобальным аттрактором.

В главе II построены обобщенные глобальные аттракторы для всех изученных в ней уравнений математической физики. Таким образом, можно сделать вывод о том, что метод траекторных аттракторов является весьма универсальным инструментом при исследовании сложных нелинейных систем математической физики. 
В главе III рассматриваются некоторые задачи об аппроксимации и возмущения траекторных аттракторов. Несмотря на то что изучено лишь небольшое число примеров приближения траекторных аттракторов для конкретных уравнений, метод исследования является достаточно общим. Это позволяет легко приспосабливать его к весьма разнообразным задачам, включая сложные случаи сингулярных приближений и возмущений.

В главе III изучены следующие проблемы: приближение 3D-системы НавьеСтокса траекторными аттракторами галёркинских приближений, аппроксимация 3D-системы Навье-Стокса $\alpha$-моделью Лерэ, задача о пределе нулевой вязкости двумерной диссипативной системы Навье-Стокса, приводящая к траекторному аттрактору соответствующей двумерной диссипативной системы Эйлера. В последнем разделе изучена специальная система реакции-диффузии с малым коэффициентом диффузии, нулевой предел которого приводит к анализу траекторного аттрактора некоторой частично диссипативной системы реакции-диффузии.

Обратим внимание на то, что в последних трех задачах малый параметр стоит при старших производных изучаемых уравнений, поэтому исследование аттракторов таких сингулярных задач другими методами сопряжено со значительными трудностями. Метод траекторных аттракторов хорошо приспособлен к решению подобных задач. Другие примеры можно найти в монографии [12].

Цель авторов при написании этой работы заключалась в пропагандировании метода траекторных аттракторов, который, наряду с другими известными подходами, позволяет наиболее глубоко продвинуться в вопросах изучения динамики решений многих сложных уравнений математической физики. Отметим, что мы не касались весьма обширной области, связанной с анализом неавтономных уравнений математической физики, где также имеются значительные продвижения (см. [12]). Авторы планируют изложить теорию траекторных аттракторов неавтономных уравнений в отдельной публикации.

Авторы выражают глубокую признательность Сергею Зелику за плодотворное обсуждение данной статьи, а также за помощь при написании раздела, относящегося к аттракторам нелинейных эллиптических уравнений в цилиндрических областях.

\section{Глава I}

\section{Глобальные аттракторы эволюционных уравнений}

В настоящей главе, которая имеет предварительный характер, изучаются глобальные аттракторы общих диссипативных полугрупп, действующих в полных метрических или банаховых пространствах. Приводится классическая схема построения полугруппы, отвечающей абстрактному автономному функциональному уравнению в банаховом пространстве, для которого справедлива теорема единственности начальной задачи Коши. Формулируются теоремы о существовании и структуре глобальных аттракторов диссипативных динамических систем. Приводятся известные примеры уравнений математической физики, для которых теорема единственности задачи Коши имеет место и для которых по классической схеме можно построить глобальные аттракторы. 


\section{1. Полугруппы и глобальные аттракторы}

Рассматривается общая (нелинейная) полугруппа $\{S(t), t \geqslant 0\}$, действующая на некотором множестве $E, S(t): E \rightarrow E$. Предполагается, что $E$ является полным метрическим или банаховым пространством.

ОПредЕЛЕНиЕ 1.1. Семейство отображений $S(t): E \rightarrow E$, зависящее от вещественного параметра $t \geqslant 0$, называется полугруппой, если оно удовлетворяет соотношениям

$$
S(0)=\mathrm{Id}, \quad S\left(t_{1}\right) S\left(t_{2}\right)=S\left(t_{1}+t_{2}\right), \quad \forall t_{1}, t_{2} \geqslant 0 .
$$

Здесь и далее Id обозначает тождественный оператор. Через $\mathscr{B}(E)$ обозначается совокупность всех множеств из $E$, ограниченных в метрике (или норме) этого метрического (или банахова) пространства.

Мы будем рассматривать диссипативные динамическим системы. В применении к соответствующим полугруппам свойство диссипативности прежде всего означает, что полугруппа обладает ограниченным поглощающим множеством.

Множество $B_{0} \subset E$ называется поглощающим множеством полугруппы $\{S(t)\}$, если для любого $B \in \mathscr{B}(E)$ найдется число $\tau=\tau(B)>0$ такое, что

$$
S(t) B \subset B_{0}, \quad \forall t \geqslant \tau .
$$

Построение ограниченного поглощающего множества является первым существенным шагом при изучении асимптотических свойств полугруппы $\{S(t)\}$, когда $t \rightarrow+\infty$. Следующие важные шаги, которые приводят к понятию глобального аттрактора, связаны с изучением компактных притягивающих множеств.

Множество $P \subset E$ называется притягивающим для полугруппы $\{S(t)\}$, если для любого $B \in \mathscr{B}(E)$

$$
\operatorname{dist}_{E}(S(t) B, P) \rightarrow 0 \quad \text { при } t \rightarrow+\infty .
$$

Величина

$$
\operatorname{dist}_{E}(X, Y)=\sup _{x \in X} \inf _{y \in Y}\|y-x\|_{E}, \quad X, Y \subseteq E,
$$

называется (несимметрическим) отклонением по Хаусдорфу множества $X$ от множества $Y$ в пространстве $E$. Соотношение (1.3) эквивалентно следующему свойству: любая $\varepsilon$-окрестность $\mathscr{O}_{\varepsilon}(P)$ множества $P$ в пространстве $E$ является поглощающим множеством для полугруппы $\{S(t)\}$, т. е. для любого $B \in \mathscr{B}(E)$ найдется $\tau=\tau(B, \varepsilon)$ такое, что

$$
S(t) B \subset \mathscr{O}_{\varepsilon}(P), \quad \forall t \geqslant \tau .
$$

При этом само множество $P$ может не являться поглощающим для $\{S(t)\}$.

Свойство диссипативности полугруппы чаще всего выражается в наличии у нее компактного поглощающего или притягивающего множества. Полугруппа 
$\{S(t)\}$ называется компактной, если для нее существует компактное в $E$ поглощающее множество $P \Subset E$. Полугруппа $\{S(t)\}$ называется асимптотически компактной, если она имеет компактное притягивающее множество $K \Subset E$.

Наиболее полно поведение полугруппы $\{S(t)\}$ при $t \rightarrow+\infty$ можно описать с помощью глобального аттрактора этой полугруппы.

ОПРЕДЕЛЕниЕ 1.2. Множество $\mathscr{A} \in \mathscr{B}(E)$ называется глобалъным аттрактором полугруппы $\{S(t)\}$, если выполнены следующие условия:

1) множество $\mathscr{A}$ компактно в $E(\mathscr{A} \Subset E)$;

2) $\mathscr{A}$ является притягивающим для $\{S(t)\}$, т. е. для любого $B \in \mathscr{B}(E)$

$$
\operatorname{dist}_{E}(S(t) B, \mathscr{A}) \rightarrow 0 \quad \text { при } t \rightarrow+\infty \text {; }
$$

3) $\mathscr{A}$ строго инвариантно относительно $\{S(t)\}$, т. е.

$$
S(t) \mathscr{A}=\mathscr{A}, \quad \forall t \geqslant 0 .
$$

Как доказано, например, в [1], глобальный аттрактор $\mathscr{A}$ полугруппы $\{S(t)\}$ является для нее минимальным компактным притягивающим множеством, и поэтому он определяется однозначно. Кроме того, множество $\mathscr{A}$ является максимальным ограниченным и строго инвариантным множеством для $\{S(t)\}$ (см. [3], [14], [15]). Это означает следующее: если $Y \in \mathscr{B}(E)$ и $S(t) Y=Y$ при всех $t \geqslant 0$, то $Y \subseteq \mathscr{A}$.

ОПРеДЕЛЕНИЕ 1.3. Пусть $B \in \mathscr{B}(E)$. Множество

$$
\omega(B)=\bigcap_{h \geqslant 0}\left[\bigcup_{t \geqslant h} S(t) B\right]_{E}
$$

называется $\omega$-предельным множеством для $B$. Здесь и далее $[Q]_{E}$ обозначает замыкание множества $Q$ в топологическом пространстве $E$.

Напомним, что полугруппа $\{S(t)\}$ называется непрерывной, если каждое отображение $S(t), t \geqslant 0$, является непрерывным в метрике пространства $E$.

Сформулируем теорему о существовании глобального аттрактора полугруппы, которая является классическим результатом в теории диссипативных динамических систем (см. [1], [2]).

Теорема 1.1. Пусть задана непрерывная полугруппа $\{S(t)\}$ в полном метрическом пространстве $E$, которая асимптотически компактна и имеет компактное притягивающее множество $K \Subset E$. Тогда для полугруппы $\{S(t)\}$ существует глобальный аттрактор $\mathscr{A}(\mathscr{A} \subseteq K)$. Множество $\mathscr{A}$ совпадает с $\omega$-предельным множеством для $K: \mathscr{A}=\omega(K)$.

ЗАмЕчАНИЕ 1.1. При выполнении условий теоремы 1.1 глобальный аттрактор $\mathscr{A}$ можно построить по формуле

$$
\mathscr{A}=\bigcap_{t \geqslant 0} S(t) P,
$$

где $P$ - любое ограниченное притягивающее множество полугруппы $\{S(t)\}$. 
Введем понятие полной траектории полугруппы $\{S(t)\}$. Кривая $u(s), s \in \mathbb{R}$, в пространстве $E$ называется полной траекторией полугруппы $\{S(t)\}$, если

$$
S(t) u(s)=u(t+s), \quad \forall s \in \mathbb{R}, \quad t \in \mathbb{R}_{+} .
$$

При описании глобальных аттракторов важную роль играет понятие ядра полугруппы.

ОПРЕДЕЛЕНИЕ 1.4. Ядро $\mathscr{K}$ полугруппы $\{S(t)\}$ состоит из всех ограниченных полных траекторий этой полугруппы:

$$
\mathscr{K}=\left\{u(\cdot) \mid u(s) \text { удовлетворяет (1.6) и }\|u(s)\|_{E} \leqslant C_{u} \text { для всех } s \in \mathbb{R}\right\} .
$$

Сечением ядра в момент времени $t \in \mathbb{R}$ называется множество

$$
\mathscr{K}(t)=\{u(t) \mid u \in \mathscr{K}\} \subset E .
$$

Следующий важный результат вытекает из свойства строгой инвариантности глобального аттрактора (см., например, [1]).

ТЕОРема 1.2. При выполнении условий теоремы 1.1 глобальный аттрактор $\mathscr{A}$ полугруппы $\{S(t)\}$ совпадает с сечением ее ядра в момент $t=0$ :

$$
\mathscr{A}=\mathscr{K}(0) .
$$

Сформулированные теоремы о существовании и структуре глобальных аттракторов являются весьма общими, и их можно применять к всевозможным динамическим системам, встречающимся в математической физике, которые обладают указанными выше свойствами диссипативности.

Весьма наглядно полугруппы (динамические системы) возникают при изучении автономных дифференциальных уравнений, которые имеют вид

$$
\partial_{t} u=-F(u),
$$

где $F$ обозначает некоторый оператор, действующий в пространстве $E$, которое, для определенности, предполагается банаховым. Если $E$ является конечномерным евклидовым пространством, то (1.9) просто является автономной системой обыкновенных дифференциальных уравнений. В данной статье мы будем в основном рассматривать бесконечномерные пространства $E$, а $(1.9)$ будет уравнением или системой уравнений с частными производными.

В уравнении (1.9) $u=u(t)$ обозначает неизвестную функцию времени $t \geqslant 0$ со значениями в пространстве $E$, а $\partial_{t} u(t)$ - это производная по времени функции $u(t)$. (Здесь мы не уточняем, в каком смысле понимается производная по времени; это зависит от вида конкретных дифференциальных уравнений, обыкновенных или с частными производными.)

Предположим, что для любого элемента $u_{0} \in E$ уравнение (1.9) имеет и притом единственное решение $u=u(t), t \geqslant 0$, с начальным условием $u(0)=u_{0}$. (Мы пока не уточняем, в каком смысле решение $u(t)$ должно удовлетворять уравнению и начальному условию.) При выполнении этого условия уравнению (1.9) можно сопоставить семейство разрешающих операторов

$$
S(t): u_{0} \rightarrow u(t)
$$


которые действуют в пространстве $E, S(t): E \rightarrow E$. Семейство операторов $\{S(t), t \geqslant 0\}$ образует полугруппу в пространстве $E$. Заметим, что этот факт сразу вытекает из предполагаемого условия однозначной разрешимости начальной задачи Коши для автономного уравнения.

При таком подходе к исследованию дифференциального уравнения (1.9) поведение его решений $u(t)$ при $t \rightarrow+\infty$ можно адекватно описать с помощью глобального аттрактора полугруппы $\{S(t)\}$, отвечающей этому уравнению. Таким методом в работах многих математиков было исследовано большое число диссипативных уравнений с частными производными различных типов (см. обзоры [16]-[19]). Некоторые важные примеры уравнений будут рассмотрены в следующем разделе. При этом всегда предполагается, что изучаемая задача Коши является корректно поставленной в том смысле, что она имеет и притом единственное решение в соответствующем функциональном пространстве.

Описанный выше метод построения эволюционной полугруппы для дифференциального уравнения является достаточно универсальным, но не единственным. Другой эффективный метод будет изложен в главе II при исследовании некоторых некорректных задач, для которых может отсутствовать свойство единственности решений соответствующей задачи Коши.

\section{2. Глобальные аттракторы уравнений математической физики}

В этом разделе рассматриваются некоторые типичные диссипативные уравнения математической физики, для которых строятся глобальные аттракторы с помощью методов, описанных в предыдущем разделе.

Начнем с достаточно простого, но вполне содержательного примера системы двух квазилинейных уравнений параболического типа. Такие системы принято называть системами реакции-диффузии или РД-системами.

Система реакции-диффузии. Задана ограниченная область $\Omega \Subset \mathbb{R}^{3}$ с кусочно гладкой границей $\partial \Omega$. Через $u(x, t)$ и $v(x, t)$ обозначаются (неизвестные) скалярные функции с аргументами $x \in \Omega, t \geqslant 0$, которые удовлетворяют следующим уравнениям:

$$
\begin{aligned}
& \partial_{t} u=a_{1} \Delta u-f_{1}(u, v), \\
& \partial_{t} v=a_{2} \Delta v-f_{2}(u, v),
\end{aligned}
$$

где $a_{1}$ и $a_{2}$ - положительные коэффициенты диффузии, $\Delta$ - оператор Лапласа в пространстве $\mathbb{R}_{x}^{3}$. Нелинейные функции взаимодействия $f_{1}(u, v)$ и $f_{2}(u, v)$ будут описаны ниже. На границе области $\partial \Omega$ задаются условия Дирихле

$$
\left.u\right|_{\partial \Omega}=0,\left.\quad v\right|_{\partial \Omega}=0 .
$$

(Аналогично, можно рассматривать другие граничные условия, например, условия Неймана.) Физический смысл неизвестных функций $u(x, t)$ и $v(x, t)-$ концентрации химических веществ, участвующих в некоторой химической реакции, в точке $x \in \Omega$ в момент времени $t \geqslant 0$. Нелинейные функции $f_{1}(u, v)$ и $f_{2}(u, v)$ определяют закон превращения веществ (реакцию), а члены $a_{1} \Delta u$ и $a_{2} \Delta v$ соответствуют диффузии этих веществ. 
Предполагается, что функции $f_{1}, f_{2}: \mathbb{R}^{2} \rightarrow \mathbb{R}$ являются непрерывными и удовлетворяют следующим условиям роста для любых $(u, v) \in \mathbb{R}^{2}$

$$
\begin{gathered}
\left|f_{1}(u, v)\right|^{p_{1} /\left(p_{1}-1\right)}+\left|f_{2}(u, v)\right|^{p_{2} /\left(p_{2}-1\right)} \leqslant C_{1}\left(|u|^{p_{1}}+|v|^{p_{2}}+1\right) \\
\gamma|u|^{p_{1}}+\sigma|v|^{p_{2}}-C_{2} \leqslant f_{1}(u, v) u+f_{2}(u, v) v
\end{gathered}
$$

где $\gamma, \sigma, C_{1}, C_{2}$ - некоторые положительные константы и $p_{1}, p_{2} \geqslant 2$. В приложениях функции $f$ и $h$ обычно бывают полиномами по $u$ и $v$ степени $p_{1}-1$ и $p_{2}-1$, что объясняет условие (2.4). Неравенство (2.5) является весьма существенным. Из него выводится диссипативность изучаемой системы.

Опишем класс функций $u(x, t)$ и $v(x, t)$, в котором ищется решение системы (2.1)-(2.3). Зафиксируем любое число $T>0$. Для произвольных функций $u(\cdot) \in L_{p_{1}}\left(0, T ; L_{p_{1}}(\Omega)\right)$ и $v(\cdot) \in L_{p_{2}}\left(0, T ; L_{p_{2}}(\Omega)\right)$ из условия $(2.4)$ вытекает, что

$$
f_{i}(u(\cdot), v(\cdot)) \in L_{q_{i}}\left(0, T ; L_{q_{i}}(\Omega)\right), \quad i=1,2,
$$

где $q_{i}=p_{i} /\left(p_{i}-1\right)$ - сопряженные показатели к $p_{i}$ при $i=1,2$. Если дополнительно известно, что $u(\cdot), v(\cdot) \in L_{2}\left(0, T ; H_{0}^{1}(\Omega)\right)$, то

$$
a_{1} \Delta u(\cdot), a_{2} \Delta v(\cdot) \in L_{2}\left(0, T ; H^{-1}(\Omega)\right) .
$$

Следовательно, для таких функций $u$ и $v$ выражения, стоящие в правых частях уравнений $(2.1)$ и $(2.2)$, принадлежат пространствам $L_{2}\left(0, T ; H^{-1}(\Omega)\right)+$ $L_{q_{1}}\left(0, T ; L_{q_{1}}(\Omega)\right)$ и $L_{2}\left(0, T ; H^{-1}(\Omega)\right)+L_{q_{2}}\left(0, T ; L_{q_{2}}(\Omega)\right)$ соответственно (см. [20]).

По определению, пара функций $(u(x, t), v(x, t)), x \in \Omega, t \geqslant 0$, называется (слабым) решением системы (2.1)-(2.3), если для любого $T>0$

$$
\begin{aligned}
& u(\cdot) \in L_{p_{1}}\left(0, T ; L_{p_{1}}(\Omega)\right) \cap L_{2}\left(0, T ; H_{0}^{1}(\Omega)\right), \\
& v(\cdot) \in L_{p_{2}}\left(0, T ; L_{p_{2}}(\Omega)\right) \cap L_{2}\left(0, T ; H_{0}^{1}(\Omega)\right)
\end{aligned}
$$

и для любой функции $\varphi(\cdot) \in L_{p_{1}}(\Omega) \cap H_{0}^{1}(\Omega)$ выполнены дифференциальное тождество

$$
\frac{d}{d t} \int_{\Omega} u(x, t) \varphi(x) d x+\int_{\Omega}\left(a_{1} \sum_{i=1}^{3} \partial_{x_{i}} u(x, t) \partial_{x_{i}} \varphi(x)+f_{1}(u(x, t), v(x, t)) \varphi(x)\right) d x=0
$$

и аналогичное тождество для функции $v(x, t)$. Это эквивалентно тому, что функции $u(x, t)$ и $v(x, t)$ удовлетворяют уравнениям $(2.1)$ и $(2.2)$ в пространствах обобщенных функций в $\mathscr{D}^{\prime}\left(0, T ; H^{-1}(\Omega)+L_{q_{1}}(\Omega)\right)$ и $\mathscr{D}^{\prime}\left(0, T ; H^{-1}(\Omega)+\right.$ $\left.L_{q_{2}}(\Omega)\right)$ соответственно (см. [1], [20], [12]). Из этих уравнений следует, что

$$
\begin{aligned}
& \partial_{t} u(\cdot) \in L_{2}\left(0, T ; H^{-1}(\Omega)\right)+L_{q_{1}}\left(0, T ; L_{q_{1}}(\Omega)\right), \\
& \partial_{t} v(\cdot) \in L_{2}\left(0, T ; H^{-1}(\Omega)\right)+L_{q_{2}}\left(0, T ; L_{q_{2}}(\Omega)\right) .
\end{aligned}
$$

Заметим, что в силу (2.10) и (2.11) функции $u(x, t)$ и $v(x, t)$ при $t \geqslant 0$ являются непрерывными со значениями в пространствах $H^{-1}(\Omega)+L_{q_{1}}(\Omega)$ и $H^{-1}(\Omega)+$ $L_{q_{2}}(\Omega)$. В частности, для любого слабого решения $(u(x, t), v(x, t))$ РД-системы 
корректно определены значения $u(x, 0)$ и $v(x, 0)$ при $t=0$. Поэтому уравнения (2.1)-(2.3) можно дополнить начальными условиями

$$
\left.u(x, t)\right|_{t=0}=u_{0}(x),\left.\quad v(x, t)\right|_{t=0}=v_{0}(x) .
$$

Для упрощения записи мы будем опускать аргумент $x$ в записи решения $(u(x, t)$, $v(x, t))$ системы $(2.1)-(2.2)$ и обозначать это решение $(u(t), v(t))$.

Сформулируем важный результат о существовании слабого глобального решения системы (2.1)-(2.3).

УтВЕРЖДЕНИЕ 2.1. Пусть выполнены условия (2.4) и (2.5). Тогда при любых $u_{0} \in L_{2}(\Omega)$ и $v_{0} \in L_{2}(\Omega)$ начальная задача Коши (2.12) для РД-системы (2.1)-(2.3) всегда имеет по крайней мере одно слабое решение $(u(x, t), v(x, t))$ в классе функиий (2.8), (2.9). При этом $u, v \in C\left(\mathbb{R}_{+} ; L_{2}(\Omega)\right)$ и справедливо неравенство

$$
\|u(t)\|_{L_{2}(\Omega)}^{2}+\|v(t)\|_{L_{2}(\Omega)}^{2} \leqslant\left(\left\|u_{0}\right\|_{L_{2}(\Omega)}^{2}+\left\|v_{0}\right\|_{L_{2}(\Omega)}^{2}\right) \mathrm{e}^{-\alpha t}+R_{0}^{2},
$$

где положительные константы $\alpha$ u $R_{0}$ не зависят от $u_{0} u v_{0}$.

Доказательство проводится с помощью классического метода галёркинских приближений (см., например, [1], [2]).

Заметим, что для построения полугруппы в фазовом пространстве $E=$ $\left[L_{2}(\Omega)\right]^{2}$ начальных условий РД-системы (2.1)-(2.3) и для построения глобального аттрактора нам не хватает теоремы единственности для решения соответствующей задачи Коши. Оказывается, условий (2.4) и (2.5) для этого недостаточно. Сформулируем простое достаточное условие, которое позволит построить полугруппу.

Предположим, что нелинейные функции $f_{1}(u, v)$ и $f_{2}(u, v)$ в системе (2.1)-(2.2) являются дифференцируемыми. Обозначим через $\mathbf{J}$ якобиан отображения $\left(f_{1}, f_{2}\right): \mathbb{R}^{2} \rightarrow \mathbb{R}^{2}$ :

$$
\mathbf{J}(u, v)=\left[\begin{array}{ll}
\partial_{u} f_{1}(u, v) & \partial_{v} f_{1}(u, v) \\
\partial_{u} f_{2}(u, v) & \partial_{v} f_{2}(u, v)
\end{array}\right] .
$$

Пусть выполнено следующее условие: для некоторого числа $D>0$ матрица $\mathbf{J}(u, v)+D \mathbf{I}$ является положительной при всех $(u, v) \in \mathbb{R}^{2}$, т. е.

$$
(\mathbf{J}(u, v) \xi, \xi)+D(\xi, \xi) \geqslant 0, \quad \forall \xi \in \mathbb{R}^{2} .
$$

УтВеРЖДЕНИЕ 2.2. При выполнении дополнительного условия (2.14) решение $(u(t), v(t))$ задачи Коши (2.12) для системы (2.1)-(2.3) является единственным. Кроме того, $u(\cdot), v(\cdot) \in C(] 0,+\infty\left[; H_{0}^{1}(\Omega)\right)$ и справедливо неравенcmвo

$$
\begin{aligned}
& \|u(t)\|_{H_{0}^{1}(\Omega)}^{2}+\|v(t)\|_{H_{0}^{1}(\Omega)}^{2} \\
& \quad \leqslant C_{2}\left(\left\|u_{0}\right\|_{L_{2}(\Omega)}^{2}+\left\|v_{0}\right\|_{L_{2}(\Omega)}^{2}\right)\left(t+t^{-1}\right) \mathrm{e}^{-\beta t}+\left(1+t^{-1}\right) R_{1}^{2}, \quad \forall t>0,
\end{aligned}
$$

где положительные константы $\beta, C_{2}$ и $R_{1}$ не зависят от $u_{0}, v_{0} \in L_{2}(\Omega)$. 
Доказательство приведено в [1], [2], [12].

Теперь, следуя схеме, изложенной в разделе I, строим полугруппу $\{S(t)\}$ в пространстве $E=\left[L_{2}(\Omega)\right]^{2}$, которая порождается изучаемой РД-системой:

$$
S(t)\left(u_{0}, v_{0}\right)=(u(t), v(t)), \quad t \geqslant 0,
$$

где $(u(t), v(t))$ - решение системы (2.1)-(2.3) с начальными условиями (2.12).

Из неравенства (2.13) следует, что построенная полугруппа имеет ограниченное в $E$ поглощающее множество, а именно шар $B_{0}$ с центром в нуле и радиусом $R>R_{0}$. Следовательно, рассматриваемая динамическая система является диссипативной. Более того, из неравенства (2.15) вытекает, что шар $B_{1}$ в пространстве $\left[H_{0}^{1}(\Omega)\right]^{2}$ с радиусом $R>2 R_{1}$ также является поглощающим множеством для полугруппы $\{S(t)\}$. Множество $B_{1}$, по теореме вложения Соболева, компактно в пространстве $\left[L_{2}(\Omega)\right]^{2}$. Следовательно, полугруппа $\{S(t)\}$ является компактной в фазовом пространстве $E$.

Для построения глобального аттрактора осталось проверить, что полугруппа $\{S(t)\}$ является непрерывной. Это вытекает из следующего известного утверждения, из которого, на самом деле, и выводится единственность решений задачи (2.1)-(2.3), (2.12). Доказательство имеется в [1], [2], [12].

УтВЕРЖДЕНИЕ 2.3. При выполнении условия (2.14) справедливо неравенство

$$
\left\|\left(u_{1}(t), v_{1}(t)\right)-\left(u_{2}(t), v_{2}(t)\right)\right\|_{\left[L_{2}(\Omega)\right]^{2}} \leqslant\left\|\left(u_{01}, v_{01}\right)-\left(u_{02}, v_{02}\right)\right\|_{\left[L_{2}(\Omega)\right]^{2}} \mathrm{e}^{D t},
$$

для любого $t \geqslant 0$, где $\left(u_{i}(t), v_{i}(t)\right)=S(t)\left(u_{0 i}, v_{0 i}\right)$ - решение системъ $(2.1)-(2.3)$ с начальными условиями $\left(u_{0 i}, v_{0 i}\right) \in\left[L_{2}(\Omega)\right]^{2}, i=1,2$, а величина $D$ та же, что в неравенстве (2.14).

Рассмотрим также ядро $\mathscr{K}$ полугруппы $\{S(t)\}$, которое состоит из решений $(u(t), v(t))$ системы $(2.1)-(2.3)$, заданных при всех $t \in \mathbb{R}$ и ограниченных по норме $\left[L_{2}(\Omega)\right]^{2}$ :

$$
\mathscr{K}=\left\{(u(t), v(t)), t \in \mathbb{R},- \text { решение }(2.1)-(2.3) \text { и }\|(u(t), v(t))\|_{\left[L_{2}(\Omega)\right]^{2}} \leqslant C_{(u, v)}\right\} .
$$

Для полугруппы $\{S(t)\}$ системы (2.1)-(2.3) проверены все условия теорем 1.1 и 1.2 , из которых получаем следующий результат.

ТЕОрема 2.1. При выполнении условий (2.4), (2.5) и (2.14) полугруппа $\{S(t)\}$, отвечающая РД-системе и действующая в пространстве $E=\left[L_{2}(\Omega)\right]^{2}$, имеет глобальный аттрактор $\mathscr{A}$, который ограничен в пространстве $\left[H_{0}^{1}(\Omega)\right]^{2}$. Кроме того, $\mathscr{A}$ совпадает с сечением ядра $\mathscr{K}$ задачи: $\mathscr{A}=\mathscr{K}(0)$.

ЗАмечАниЕ 2.1. Теория диссипативных РД-систем развивается значительными темпами. Упомянем лишь некоторые классические системы, относящиеся к виду (2.1), (2.2). Это модельное уравнение Чефи-Инфанте (одно скалярное уравнение), уравнения ФитцХью-Нагумо, системы, отвечающие реакции Белоусова-Жаботинского и ее многочисленным обобщениям, системы Лотка-Вольтерра с диффузией в теоретической экологии и многие другие системы (см. [21], [22]). 
Метод разрешающих полугрупп и их глобальных аттракторов вносит дополнительную математическую строгость в данную область научных знаний (см. также [2], [12], [23]). Однако стоит отметить, что ключевое свойство типа неравенства (2.14), необходимое для корректности задачи и для построения глобального аттрактора, в реальных РД-системах может отсутствовать. Это приводит к необходимости искать обобщение теории глобальных аттракторов для адекватного и строгого математического описания эволюции сложных РД-систем. Это можно сделать, например, с помощью теории траекторных аттракторов, которая излагается в следующих главах.

Ниже кратко рассматриваются другие важные диссипативные системы математической физики и их глобальные аттракторы.

Двумерная система Навье-Стокса. Эти уравнения являются весьма популярным примером при изучении глобальных аттракторов. С этой системы по сути начались систематические исследования бесконечномерных динамических систем в пионерских работах О.А. Ладыженской (см. [14], [15]) и ее последователей (см. обзор [24]).

Рассматривается 2D-система Навье-Стокса в ограниченной области $\Omega \Subset \mathbb{R}^{2}$ :

$$
\begin{gathered}
\partial_{t} u+\sum_{i=1}^{2} u^{i} \partial_{x_{i}} u=\nu \Delta u-\nabla p+g(x), \\
(\nabla, u):=\partial_{x_{1}} u^{1}+\partial_{x_{2}} u^{2}=0,\left.\quad u\right|_{\partial \Omega}=0,
\end{gathered}
$$

где вектор $u=u(x, t)=\left(u^{1}(x, t), u^{2}(x, t)\right)$ является скоростью, а скаляр $p=$ $p(x, t)$ - давлением жидкости в точке $x=\left(x_{1}, x_{2}\right) \in \Omega$ в момент времени $t \geqslant 0$. Коэффициент вязкости $\nu>0$ и внешняя сила $g(x)=\left(g^{1}(x), g^{2}(x)\right)$ считаются известными.

Вводятся обозначения: $H$ и $V=H^{1}$ - замыкания множества

$$
\mathscr{V}=\left\{v \mid v \in\left(C_{0}^{\infty}(\Omega)\right)^{2},(\nabla, v)=0\right\}
$$

в нормах $|\cdot|$ и $\|\cdot\|$ пространств $\left[L_{2}(\Omega)\right]^{2}$ и $\left[H_{0}^{1}(\Omega)\right]^{2}$ соответственно. Напомним, что

$$
|u|^{2}:=\sum_{i=1}^{2} \int_{\Omega}\left|u^{i}(x)\right|^{2} d x, \quad\|u\|^{2}:=|\nabla u|^{2}=\sum_{i=1}^{2} \int_{\Omega}\left|\nabla u^{i}(x)\right|^{2} d x .
$$

Через $P$ обозначается ортогональный проектор $\left(L_{2}(\Omega)\right)^{2}$ на $H$, который называется оператором Лерэ. Обозначим $V^{\prime}:=V^{*}$ сопряженное пространство к $V$.

Исключая давление $p$ с помощью применения к обеим частям (2.16) оператора $P$, приводим эту систему к виду

$$
\partial_{t} u+\nu L u+B(u, u)=g(x),
$$

где $L:=-P \Delta$ и $B(u, v):=P \sum_{i=1}^{2} u^{i} \partial_{x_{i}} v$. Предполагается, что $g(\cdot) \in H$. 
Оператор Стокса $L$, рассматриваемый на области определения $V \cap\left(H^{2}(\Omega)\right)^{2}$, является положительно определенным и самосопряженным.

Начальное условие для (2.17) задается при $t=0$ :

$$
\left.u\right|_{t=0}=u_{0}(x), \quad u_{0} \in H .
$$

Отметим, что $L$ является ограниченным оператором из $V$ в $V^{\prime}$.

Рассмотрим трехлинейную непрерывную на $V$ форму $b$ :

$$
b(u, v, w):=(B(u, v), w)=\int_{\Omega} \sum_{i, j=1}^{2} u^{i} \partial_{x_{i}} v^{j} w^{j} d x .
$$

Оператор $B$ отображает $V \times V$ в $V^{\prime}$. Форма $b$ удовлетворяет тождествам

$$
b(u, v, v)=0, \quad b(u, v, w)=-b(u, w, v), \quad \forall u, v, w \in V .
$$

Кроме того, выполнено неравенство

$$
|b(u, u, v)| \leqslant c_{0}^{2}|u|\|u\|\|v\|, \quad \forall u, v \in V
$$

(см., например, [25], [26]). Это неравенство следует из оценки

$$
\|f\|_{L_{4}(\Omega)} \leqslant c|f|^{1 / 2}|\nabla f|^{1 / 2}, \quad f \in H_{0}^{1}(\Omega),
$$

при $c_{0}=c$. Заметим, что константа $c$ не зависит от $\Omega$. Неравенство $(2.21)$ часто называют неравенством Ладыженской. Из (2.20) получаем оценку

$$
|B(u, u)|_{V^{\prime}} \leqslant c_{0}^{2}\|u\||u| .
$$

Таким образом, если $u \in L_{2}(0, T ; V) \cap L_{\infty}(0, T ; H)$, то $-\nu L u-B(u, u)+g(x) \in$ $L_{2}\left(0, T ; V^{\prime}\right)$. Следовательно, уравнение $(2.17)$ можно рассматривать в смысле теории обобщенных функций из пространства $\mathscr{D}^{\prime}\left(0, T ; V^{\prime}\right)$, причем производная по времени $\partial_{t} u$ принадлежит $L_{2}\left(0, T ; V^{\prime}\right)$.

УТВЕРЖДЕНИЕ 2.4. Задача (2.17), (2.18) имеет и притом единственное решение $u(\cdot) \in C\left(\mathbb{R}_{+} ; H\right) \cap L_{2}^{\text {loc }}\left(\mathbb{R}_{+} ; V\right)$. При этом $\partial_{t} u(\cdot) \in L_{2}^{\text {loc }}\left(\mathbb{R}_{+} ; V^{\prime}\right)$ и имеют место следующие неравенства:

$$
\begin{gathered}
|u(t)|^{2} \leqslant|u(0)|^{2} \mathrm{e}^{-\nu \lambda t}+\nu^{-2} \lambda^{-2}|g|^{2} \\
|u(t)|^{2}+\nu \int_{0}^{t}\|u(s)\|^{2} d s \leqslant|u(0)|^{2}+t \nu^{-1} \lambda^{-1}|g|^{2}, \\
t\|u(t)\|^{2} \leqslant C\left(t,|u(0)|^{2}\right),
\end{gathered}
$$

где $\lambda=\lambda_{1}$ - первое собственное значение оператора Cтокса L, а функиия $C(z, R)$ является непрерывной и монотонной по z $u R$.

Сформулированное утверждение является классическим результатом о существовании и единственности решения задачи Коши для двумерной системы Навье-Стокса. Подробные доказательства можно найти, например, в [1], [20], [25]-[27]. 
Следовательно, имеется полугруппа $\{S(t)\}$, соответствующая задаче $(2.17)$, (2.18) и действующая в пространстве $H, S(t): H \rightarrow H$ при $t \geqslant 0$, по формуле

$$
S(t) u_{0}(\cdot)=u(\cdot, t)
$$

где $u(t)$ - решение начальной задачи $(2.17),(2.18)$. Очевидно, что построенное семейство отображений $\{S(t)\}$ удовлетворяет (1.1), т. е. оно образует полугруппу.

УтВеРЖДЕНИЕ 2.5. Полугруппа $\{S(t)\}$, соответствующая (2.17), (2.18) и действующая в пространстве $H$, является компактной и непрерывной.

Доказательство дано, например, в [1], [2]. Существование ограниченного поглощающего множества следует из (2.22). Компактность полугруппы $\{S(t)\}$ вытекает из свойства сглаживания (2.24). Из этих фактов получаем, что полугруппа $\{S(t)\}$ удовлетворяет условиям теорем 1.1 и 1.2 .

Теорема 2.2. Полугруппа $\{S(t)\}$, соответствующая задаче (2.17), (2.18), имеет глобальный аттрактор $\mathscr{A}$, который совпадает с сечением ядра $\mathscr{K}$ этого уравнения: $\mathscr{A}=\mathscr{K}(0)$.

Отметим, что в теоретической гидромеханике весьма важным является изучение трехмерной системы Навье-Стокса, которая аналогична двумерной системе $(2.16)$, но задана в области $\Omega \Subset \mathbb{R}^{3}$. Из классических работ Хопфа, Лерэ и Лионса известно, как строить глобальные слабые решения для $3 \mathrm{D}$-системы Навье-Стокса в соответствующем функциональном пространстве. Однако, несмотря на усилия многих математиков, доказать единственность слабого решения соответствующей задачи Коши не удается. А как было показано в разделе 1, без этого свойства нельзя задать полугруппу, а также найти для нее глобальный аттрактор. Однако выход из этой, казалось бы, тупиковой ситуации имеется, если воспользоваться теорией траекторных аттракторов, которая будет изложена в главе II и применена к 3D-системе Навье-Стокса.

Волновое уравнение с диссипацией. С этим уравнением связано начало плодотворных исследований диссипативных бесконечномерных динамических систем, полугруппы которых являются не компактными, но лишь асимптотически компактными.

Рассматривается гиперболическое уравнение

$$
\partial_{t}^{2} u+\gamma \partial_{t} u=\Delta u-f(u)+g(x),\left.\quad u\right|_{\partial \Omega}=0, \quad x \in \Omega \Subset \mathbb{R}^{n},
$$

содержащее диссипативное слагаемое $\gamma \partial_{t} u$ с параметром $\gamma>0$. Предполагается, что $g(\cdot) \in L_{2}(\Omega)$ и нелинейная функция $f(v) \in C^{1}(\mathbb{R})$ удовлетворяет условиям

$$
\begin{gathered}
F(v) \geqslant-m v^{2}-C_{m}, \quad F(v)=\int_{0}^{v} f(w) d w, \\
f(v) v-\gamma_{1} F(v)+m v^{2} \geqslant-C_{m}, \quad \forall v \in \mathbb{R},
\end{gathered}
$$

где $m>0, \gamma_{1}>0$ и $m$ достаточно мало $\left(m<\lambda_{1}\right.$, где $\lambda_{1}-$ первое собственное значение оператора $-\Delta$ при нулевых граничных условиях). 
ЗАмЕчАниЕ 2.2. Условия (2.27) и (2.28) выполнены, например, если известно, что

$$
\liminf _{|v| \rightarrow \infty} \frac{F(v)}{v^{2}} \geqslant 0, \quad \liminf _{|v| \rightarrow \infty} \frac{f(v) v-\gamma_{1} F(v)}{v^{2}} \geqslant 0 .
$$

Предполагается, что

$$
\left|f^{\prime}(v)\right| \leqslant C_{0}\left(1+|u|^{\rho}\right)
$$

где $\rho \geqslant 0$, причем $\rho \leqslant 2 /(n-2)$ при $n \geqslant 3$ и $\rho$ - любое при $n=1,2$.

Субкритический случай $\rho<2 /(n-2)$ для уравнения $(2.26)$ изучался в [28], [29] и в других работах. Критический случай $\rho=2 /(n-2)$ рассматривался в [1], [30], [31] (см. также [32], [33], [34]).

ЗАмечАниЕ 2.3. Нелинейные волновые уравнения вида (2.26) встречаются во многих разделах физики. Например, в релятивистской квантовой механике используется уравнение с нелинейной функцией $f(u)=|u|^{\rho} u$. В этом случае, очевидно, $F(u)=|u|^{\rho+2} /(\rho+2)$, и неравенство $(2.29)$ выполнено при $\gamma_{1}=$ $1 /(\rho+2)$ (см. [2] и имеющиеся там ссылки).

Из (2.30) следует, что

$$
|f(v)| \leqslant C_{1}\left(1+|u|^{\rho+1}\right) .
$$

По теореме вложения Соболева

$$
H_{0}^{1}(\Omega) \subset L_{2(\rho+1)}(\Omega)
$$

Для $n=1,2$ это вложение справедливо при любом $\rho$. Для $n \geqslant 3$, согласно сделанному предположению относительно $\rho$, имеем, что $2(\rho+1) \leqslant 2 n /(n-2)$, где число $2 n /(n-2)$ является критическим показателем, при котором имеет место теорема вложения Соболева.

Пусть имеется функция $u(\cdot) \in L_{\infty}\left(0, T ; H_{0}^{1}(\Omega)\right)$ с производной по времени $\partial_{t} u(\cdot) \in L_{\infty}\left(0, T ; L_{2}(\Omega)\right)$. Тогда $\Delta u(\cdot) \in L_{\infty}\left(0, T ; H^{-1}(\Omega)\right)$ и, согласно $(2.32)$, $f(u(\cdot)) \in L_{\infty}\left(0, T ; L_{2}(\Omega)\right)$. Следовательно,

$$
-\gamma \partial_{t} u+\Delta u-f(u)+g(x) \in L_{\infty}\left(0, T ; H^{-1}(\Omega)\right),
$$

и уравнение (2.26) можно рассматривать в пространстве $\mathscr{D}^{\prime}\left(0, T ; H^{-1}(\Omega)\right)$ обобщенных функций со значениями в $H^{-1}(\Omega)$. В частности, $\partial_{t}^{2} u(\cdot) \in L_{\infty}(0, T$; $\left.H^{-1}(\Omega)\right)$ (см. [20]).

Для уравнения (2.26) следует задать два начальных условия при $t=0$ :

$$
\left.u\right|_{t=0}=u_{0}(x),\left.\quad \partial_{t} u\right|_{t=0}=p_{0}(x) .
$$

УтВЕРЖДЕНИЕ 2.6. Если $u_{0}(\cdot) \in H_{0}^{1}(\Omega)$ u $p_{0}(\cdot) \in L_{2}(\Omega)$, то при сбормулированных выше условиях задача (2.26), (2.33) имеет и притом единственное решение $u(\cdot) \in C\left(\mathbb{R}_{+} ; H_{0}^{1}(\Omega)\right), \partial_{t} u(\cdot) \in C\left(\mathbb{R}_{+} ; L_{2}(\Omega)\right)$, причем $\partial_{t}^{2} u(\cdot) \in$ $L_{\infty}\left(\mathbb{R}_{+} ; H^{-1}(\Omega)\right)$. 
Для краткости введем обозначения $y(t)=\left(u(t), \partial_{t} u(t)\right)=(u(t), p(t)), y_{0}=$ $\left(u_{0}, p_{0}\right)=y(0)$. Через $E$ обозначается пространство вектор-функций $y(x)=$ $(u(x), p(x)), x \in \Omega$, обладающих конечной энергетической нормой

$$
\|y\|_{E}^{2}:=\|u\|_{H_{0}^{1}(\Omega)}^{2}+\|p\|_{L_{2}(\Omega)}^{2}=\int_{\Omega}\left(|\nabla u(x)|^{2}+|p(x)|^{2}\right) d x<\infty
$$

в пространстве $E=H_{0}^{1}(\Omega) \times L_{2}(\Omega)$. Тогда $y(t) \in E$ при $t \geqslant 0$ и $y(\cdot) \in C\left(\mathbb{R}_{+} ; E\right)$.

Существование и единственность решения задачи $(2.26),(2.33)$ в пространстве $E$, а также свойства этого решения установлены в [1], [2], [4], [20].

Задача (2.26), (2.33) эквивалентна следующей системе:

$$
\left\{\begin{array} { l } 
{ \partial _ { t } u = p , } \\
{ \partial _ { t } p = - \gamma p + \Delta u - f ( u ) + g , }
\end{array} \quad \left\{\begin{array}{l}
\left.u\right|_{t=0}=u_{0}, \\
\left.p\right|_{t=0}=p_{0}
\end{array}\right.\right.
$$

которую можно кратко записать в виде задачи Коши

$$
\partial_{t} y=A(y),\left.\quad y\right|_{t=0}=y_{0} .
$$

Таким образом, если $y_{0} \in E$, то задача $(2.26),(2.33)$ имеет единственное решение $y(t) \in C\left(\mathbb{R}_{+} ; E\right)$. Отсюда следует, что однозначно определена полугруппа $\{S(t)\}$ в пространстве $E: S(t) y_{0}=y(t)$ при $t \geqslant 0$.

УтвеРЖДЕНИЕ 2.7. Полугруппа $\{S(t)\}$, соответствующая начальной задаче $(2.26),(2.33)$ и действующая в пространстве E, является асимптотически компактной и непрерывной.

Доказательство приведено, например, в [1], [2], [4]. Заметим, что построенная полугруппа не является компактной, что характерно для гиперболических уравнений, решения которых не обладают свойством сглаживания. Из утверждения 2.7 и теоремы 1.1 вытекает следующий результат.

Теорема 2.3. Полугруппа $\{S(t)\}$ задачи (2.26), (2.33) имеет глобальный аттрактор $\mathscr{A}$, совпадающий с сечением ядра этого уравнения: $\mathscr{A}=\mathscr{K}(0)$.

Опять необходимо обратить внимание на то, что приведенные результаты справедливы при условии, что в неравенстве (2.30) показатель $\rho$ не превосходит некоторой величины. Например, в случае $\Omega \subset \mathbb{R}^{3}$ этот показатель не выше 2, т. е. в уравнении (2.26) допускаются нелинейные функции, например, полиномы, степени которых не выше 3. Значит, для более высоких степеней, которые интересны в конкретных физических задачах, нельзя воспользоваться теорией глобальных аттракторов при анализе предельной динамики гиперболического уравнения с диссипацией. Все эти трудности можно преодолеть, воспользовавшись понятием траекторного аттрактора (см. главу II).

Уравнение Гинзбурга-Ландау. Это уравнение весьма близко к представленной выше РД-системе. Мы его рассматриваем отдельно ввиду важности новых динамических эффектов, которые обнаруживаются при изучении этой системы. Уравнения эти встречается во многих моделях теоретической физики и механики (см., например, [35], [36], а также обзор [37]). 
Комплексное уравнение Гинзбурга-Ландау имеет вид

$$
\partial_{t} u=(1+\alpha i) \Delta u+R u-(1+i \beta)|u|^{2} u, \quad x \in \Omega \Subset \mathbb{R}^{n} .
$$

Рассматриваются периодические граничные условия в области $\Omega=] 0,2 \pi\left[{ }^{n}\right.$ или нулевые граничные условия $\left.u\right|_{\partial \Omega}=0$ в произвольной ограниченной области $\Omega \Subset \mathbb{R}^{n}$ с достаточно гладкой границей.

В уравнении (2.35) неизвестной является комплексная функция $u=u^{1}+i u^{2}$, $\alpha, \beta \in \mathbb{R}$ - это заданные дисперсионные параметры, а $R>0$ - параметр неустойчивости. Для вектор-функции $\mathbf{u}=\left(u^{1}, u^{2}\right)^{\top}$ получаем следующую систему:

$$
\left\{\begin{array}{l}
\partial_{t} u^{1}=\Delta u^{1}-\alpha \Delta u^{2}+R u^{1}-\left(\left|u^{1}\right|^{2}+\left|u^{1}\right|^{2}\right)\left(u^{1}-\beta u^{2}\right), \\
\partial_{t} u^{2}=\alpha \Delta u^{1}+\Delta u^{2}+R u^{2}-\left(\left|u^{1}\right|^{2}+\left|u^{1}\right|^{2}\right)\left(\beta u^{1}+u^{2}\right),
\end{array}\right.
$$

которую можно записать в более компактном виде:

$$
\partial_{t} \mathbf{u}=\mathbf{a} \Delta \mathbf{u}+R \mathbf{u}-\mathbf{f}(\mathbf{u})
$$

с матрицей $\mathbf{a}=\left(\begin{array}{cc}1 & -\alpha \\ \alpha & 1\end{array}\right)$ и вектор-функцией $\mathbf{f}(\mathbf{u})=|\mathbf{u}|^{2}\left(\begin{array}{cc}1 & -\beta \\ \beta & 1\end{array}\right) \mathbf{u}$.

Найдем якобиан функции $\mathbf{f}(\mathbf{u})$ :

$$
\mathbf{f}_{\mathbf{u}}(\mathbf{u})=\left(\begin{array}{cc}
3\left(u^{1}\right)^{2}-2 \beta\left(u^{1}\right)\left(u^{2}\right)+\left(u^{2}\right)^{2} & -\beta\left(u^{1}\right)^{2}+2\left(u^{2}\right)\left(u^{1}\right)-3 \beta\left(u^{2}\right)^{2} \\
3 \beta\left(u^{1}\right)^{2}+2\left(u^{2}\right)\left(u^{1}\right)+\beta\left(u^{2}\right)^{2} & \left(u^{1}\right)^{2}+2 \beta\left(u^{1}\right)\left(u^{2}\right)+3\left(u^{2}\right)^{2}
\end{array}\right) .
$$

Обозначим через $B$ матрицу билинейной формы, соответствующую матрице в правой части (2.38):

$$
B=\left(\begin{array}{cc}
3\left(u^{1}\right)^{2}-2 \beta\left(u^{1}\right)\left(u^{2}\right)+\left(u^{2}\right)^{2} & \beta\left(u^{1}\right)^{2}+2\left(u^{2}\right)\left(u^{1}\right)-\beta\left(u^{2}\right)^{2} \\
\beta\left(u^{1}\right)^{2}+2\left(u^{2}\right)\left(u^{1}\right)-\beta\left(u^{2}\right)^{2} & \left(u^{1}\right)^{2}+2 \beta\left(u^{1}\right)\left(u^{2}\right)+3\left(u^{2}\right)^{2}
\end{array}\right) .
$$

Предполагается, что

$$
|\beta| \leqslant \sqrt{3}
$$

Легко видеть, что при этом условии диагональные элементы матрицы $B$ неотрицательны, ее детерминант имеет вид

$$
\operatorname{det} B=\left(3-\beta^{2}\right)\left(\left(u^{1}\right)^{2}+\left(u^{2}\right)^{2}\right)=\left(3-\beta^{2}\right)|\mathbf{u}|^{4} \geqslant 0
$$

и, следовательно, для якобиана функции $\mathbf{f}$ выполнено неравенство

$$
\mathbf{f}_{\mathbf{u}}(\mathbf{u}) \mathbf{v} \cdot \mathbf{v} \geqslant 0, \quad \forall \mathbf{u}, \mathbf{v} \in \mathbb{R}^{2}
$$

Для пространств комплексных функций введем следующие обозначения:

$$
\mathbf{H}=L_{2}(\Omega ; \mathbb{C}), \quad \mathbf{V}=H_{0}^{1}(\Omega ; \mathbb{C}), \quad \mathbf{L}_{4}=L_{4}(\Omega ; \mathbb{C})
$$

Задача Коши для уравнения (2.37) с начальными данными

$$
\left.\mathbf{u}\right|_{t=0}=\mathbf{u}_{0}(x), \quad \mathbf{u}_{0}(\cdot) \in \mathbf{H},
$$


имеет единственное (слабое) решение $\mathbf{u}(t):=\mathbf{u}(x, t)$ в пространстве

$$
\mathbf{u}(\cdot) \in C\left(\mathbb{R}_{+} ; \mathbf{H}\right) \cap L_{2}^{\text {loc }}\left(\mathbb{R}_{+} ; \mathbf{V}\right) \cap L_{4}^{\text {loc }}\left(\mathbb{R}_{+} ; \mathbf{L}_{4}\right),
$$

причем функция $\mathbf{u}(t)$ удовлетворяет (2.37) в смысле обобщенных функций в пространстве $\mathscr{D}^{\prime}\left(\mathbb{R}_{+} ; \mathbf{H}^{-1}+\mathbf{L}_{4 / 3}\right)$. Из уравнения $(2.37)$ вытекает, что $\partial_{t} \mathbf{u}(\cdot) \in$ $L_{2}\left(0, T ; \mathbf{H}^{-1}\right)+L_{4 / 3}\left(0, T ; \mathbf{L}_{4 / 3}\right)$ при любом $T>0$. Существование такого решения $\mathbf{u}(t)$ устанавливается, например, по методу Галёркина (см. [1], [2], [12]). Доказательство теоремы единственности достаточно стандартно и основывается на неравенстве (2.40). (Отметим, что при нарушении (2.40) теорема единственности при $n \geqslant 3$ и при произвольных дисперсионных параметрах $\alpha$ и $\beta$ остается недоказанной (см. [37]-[39], где единственность доказана для важных частных случаев).

При выполнении условия (2.39) система (2.37) порождает полугруппу $\{S(t)\}$ в пространстве $\mathbf{H}$. Эта полугруппа является непрерывной и компактной (см., например, [2], [12]). По теореме 1.1 у полугруппы $\{S(t)\}$ имеется глобальный аттрактор $\mathscr{A}$, который описывает поведение решений уравнения Гинзбурга-Ландау, когда время стремится к бесконечности. Глобальный аттрактор $\mathscr{A}$ может иметь весьма сложную структуру при определенных параметрах (см., напримep, [2], [40]).

Рассмотрим отдельно случай $|\beta|>\sqrt{3}$, в котором условие (2.40) не выполнено. Для малых размерностей $n=1,2$ построение полугруппы и ее глобального аттрактора в пространстве $\mathbf{H}=\left(L_{2}(\Omega)\right)^{2}$ возможно (см. [2], [41]). При $n \geqslant 3$ можно построить глобальный аттрактор в фазовом пространстве $\mathbf{L}_{p}=\left(L_{p}(\Omega)\right)^{2}, p>n$, при условии, что $(\alpha, \beta) \in \mathscr{P}(n)$, где $\mathscr{P}(n)-$ некоторое подмножество $\mathbb{C}$ (см. [37], [40], [42], [43]).

Таким образом, без выполнения условия (2.40), которое обеспечивается неравенством $|\beta| \leqslant \sqrt{3}$, построить глобальный аттрактор с помощью методов, изложенных в этой главе, не удается. В следующей главе будет изложен другой метод, основанный на построении траекторных аттракторов, который позволит решить эту проблему (см. также [12], [44]). Траекторный аттрактор будет построен для уравнения Гинзбурга-Ландау с произвольными параметрами $n$, $\alpha, \beta$ (см. также [12], [44], [45]).

\section{Глава II \\ Траекторные аттракторы уравнений математической физики}

В этой главе излагается теория траекторных аттракторов эволюционных уравнений, для которых соответствующая задача Коши имеет глобальное решение, но при этом теорема о единственности решения может быть не установлена или не иметь места. В данной ситуации нельзя воспользоваться классической схемой построения динамической системы в фазовом пространстве начальных условий задачи Коши, изложенной в главе I, и найти глобальный аттрактор. Однако для таких уравнений можно построить траекторную динамическую систему и исследовать ее траекторный аттрактор. В первом разделе 
приводится довольно подробный анализ траекторной динамической системы для модельной системы обыкновенных дифференциальных уравнений, для которой все необходимые конструкции имеют весьма простые обоснования, что не затеняет универсальную схему построения траекторных аттракторов для более общих нелинейных уравнений с частными производными, изучение которых составляет основное содержание данной главы. В разделах 2-7 траекторные аттракторы строятся для следующих уравнений и систем: для общей диссипативной системы реакции-диффузии, для двумерной системы Эйлера с диссипацией, для трехмерной системы Навье-Стокса, для диссипативных волновых уравнений и для нелинейных эллиптических уравнений в цилиндрических областях.

\section{1. Траекторный аттрактор для модельной системы ОДУ}

Рассматривается система обыкновенных дифференциальных уравнений

$$
\partial_{t} u=-F(u), \quad t \geqslant 0,
$$

где $u=\left(u^{1}, \ldots, u^{N}\right) \in \mathbb{R}^{N}$ и функция $F(u)=\left(F^{1}(u), \ldots, F^{N}(u)\right)$ является непрерывной во всем пространстве $\mathbb{R}^{N}, F(u) \in C\left(\mathbb{R}^{N} ; \mathbb{R}^{N}\right)$. (Отметим, что дифференцируемость функции $F(u)$ не требуется.)

Мы будем исследовать решения $u=u(t), t \geqslant 0$, системы (1.1), которые понимаются в самом обычном смысле, т. е. ищутся непрерывно дифференцируемые $N$-мерные вектор-функции $u(t), t \in[0, \tau)$ при $\tau>0$, для которых производная по времени $\partial_{t} u(t)$ удовлетворяет (1.1) при всех $t \in[0, \tau)$. Классическая теорема утверждает, что для любого вектора $u_{0} \in \mathbb{R}^{N}$ существует по крайней мере одно решение $u(t), t \in[0, \tau)$, системы ОДУ, для которого

$$
u(0)=u_{0}
$$

(см., например, [46]), где $\tau=\tau\left(u_{0}\right)>0$ - максимально возможное время существования такого решения. Нас интересуют глобальные решения системы (1.1), для которых $\tau=+\infty$.

Дополнительно предполагается, что функция $F$ удовлетворяет следующему ключевому условию диссипативности:

$$
F(v) \cdot v=\sum_{i=1}^{N} F^{i}(v) v^{i} \geqslant-C+\delta|v|^{2}, \quad \forall v \in \mathbb{R}^{N}, \quad \delta>0 .
$$

Из условия диссипативности (1.3) легко выводится, что любое решение $u(t)$ уравнения (1.1) удовлетворяет неравенству

$$
|u(t)|^{2} \leqslant|u(0)|^{2} \mathrm{e}^{-2 \delta t}+\frac{C}{\delta}, \quad \forall t \in[0, \tau) .
$$

Из этого неравенства следует, что $\tau\left(u_{0}\right)=+\infty$ при каждом $u_{0} \in \mathbb{R}^{N}$, т. е. любое решение системы ОДУ (1.1) является глобальным (см. [46]).

Предположим сначала, что вектор-функция $F$ удовлетворяет локальному условию Липшица, т. е. неравенство

$$
\left|F\left(v_{1}\right)-F\left(v_{2}\right)\right| \leqslant K\left|v_{1}-v_{2}\right|
$$


выполнено при всех $v_{1}, v_{2} \in \mathbb{R}^{N},\left|v_{1}\right|,\left|v_{2}\right| \leqslant M$, для некоторого $K=K(M)>0$ (например, функция $F(u)$ является непрерывно дифференцируемой в $\mathbb{R}^{N}$ ). Из условия Липшица (1.5) выводится, что для любых двух решений $u_{1}(t)$ и $u_{2}(t)$ системы ОДУ (1.1) справедливо неравенство

$$
\left|u_{1}(t)-u_{2}(t)\right| \leqslant\left|u_{1}(0)-u_{2}(0)\right| \mathrm{e}^{K_{1} t}, \quad \forall t \geqslant 0,
$$

где $\left|u_{1}(0)\right|,\left|u_{2}(0)\right| \leqslant M$ и $K_{1}=K_{1}(M)$. Тогда при любом $u_{0} \in \mathbb{R}^{N}$ решение $u(t)$ задачи Коши (1.1)-(1.2) является единственным. В этом случае можно воспользоваться схемой, изложенной в разделе 1 главы I, и построить полугруппу $\{S(t)\}$ в пространстве $\mathbb{R}^{N}$, отвечающую задаче (1.1)-(1.2):

$$
S(t): \mathbb{R}^{N} \rightarrow \mathbb{R}^{N}, \quad S(t) u_{0}=u(t), \quad t \geqslant 0 .
$$

Кроме того, из неравенства (1.4) следует, что шар $B_{0}=\left\{|u|^{2} \leqslant 2 C / \delta\right\}$ является поглощающим множеством для $\{S(t)\}$. Из неравенства (1.6) заключаем, что полугруппа $\{S(t)\}$ непрерывна в $\mathbb{R}^{N}$. Поглощающее множество $B_{0}$ компактно в $\mathbb{R}^{N}$. Следовательно, по теореме 1.1 главы I у полугруппы $\{S(t)\}$ имеется глобальный аттрактор $\mathscr{A} \Subset \mathbb{R}^{N}$, который описывает динамику всех решений системы (1.1) при $t \rightarrow+\infty$. Таков традиционный подход к исследованию динамической системы, отвечающей уравнению (1.1), если функция $F(u)$ является достаточно гладкой.

Предположим теперь, что в уравнении (1.1) функция $F(u)$ принадлежит $C\left(\mathbb{R}^{N} ; \mathbb{R}^{N}\right)$, выполнено неравенство (1.3), а условия Липшица нет. Примером может служить ОДУ

$$
\partial_{t} u=-2|u|^{1 / 2}(u-1) .
$$

В этом случае теорема единственности решений задачи Коши (1.1)-(1.2) может нарушаться, что не позволяет построить по формуле (1.7) однозначную полугруппу $\{S(t)\}$ в фазовом пространстве $\mathbb{R}^{N}$ начальных условий. Однако возможен иной подход к исследованию эволюции решений таких уравнений.

Фазовое пространство траекторий и его свойства. Для системы (1.1) будет построена другая однозначная полугруппа, действующая в фазовом пространстве, которое отличается от $\mathbb{R}^{N}$.

В функциональном пространстве $C^{1}\left(\mathbb{R}_{+} ; \mathbb{R}^{N}\right)$ рассматривается множество $\mathscr{K}^{+}$, которое состоит из всех глобальных решений системы (1.1), т. е.

$$
\mathscr{K}^{+}=\left\{u(\cdot) \in C^{1}\left(\mathbb{R}_{+} ; \mathbb{R}^{N}\right) \mid \partial_{t} u(t)=-F(u(t)), \forall t \geqslant 0\right\} .
$$

Множество $\mathscr{K}^{+}$называется пространством траекторий системы (1.1). Элементы пространства $\mathscr{K}^{+}$(траектории) мы будем обозначать $u(s), s \geqslant 0$, заменяя буквой $s$ переменную времени $t$. Ясно, что множество $\mathscr{K}^{+}$достаточно велико, поскольку, как было отмечено, задача Коши (1.1)-(1.2) разрешима при любом $u_{0} \in \mathbb{R}^{N}$. Множество $\mathscr{K}^{+}$будет служить новым фазовым пространством.

Зададим полугруппу на $\mathscr{K}^{+}$. В пространстве $C^{1}\left(\mathbb{R}_{+} ; \mathbb{R}^{N}\right)$ действуют операторы трансляций $T(h), h \geqslant 0$, которые сдвигают аргумент $s$ вдоль оси времени на интервал $h$ :

$$
T(h) y(s)=y(s+h), \quad s \geqslant 0 .
$$


Легко проверить, что семейство операторов $\{T(h)\}, h \geqslant 0$, образует полугруппу, т. е. $T(0)=\operatorname{Id}$ и $T\left(h_{1}\right) T\left(h_{2}\right)=T\left(h_{1}+h_{2}\right)$ при любых $h_{1}, h_{2} \geqslant 0$.

Рассмотрим действие полугруппы трансляций $\{T(h)\}$ на пространстве траекторий $\mathscr{K}^{+}$. Если $u(\cdot) \in \mathscr{K}^{+}$, т. е. $u(t), t \geqslant 0$, является решением (1.1), то функция $T(h) u(t)=u(t+h), t \geqslant 0$, также является решением этого уравнения при любом $h \geqslant 0$, так как уравнение является автономным. Следовательно,

$$
T(h): \mathscr{K}^{+} \rightarrow \mathscr{K}^{+}, \quad \forall h \geqslant 0 .
$$

Для удобства мы будем обозначать полугруппу трансляций $\{T(h)\}, h \geqslant 0$, через $\{T(t)\}, t \geqslant 0$. Параметр $t$ соответствует эволюции по времени решений системы (1.1), изучаемых при $t \rightarrow+\infty$.

Построенная полугруппа $\{T(t)\}$ является однозначной на фазовом пространстве $\mathscr{K}^{+}$. Отсутствие единственности решений задачи Коши (1.1)-(1.2) этому не препятствует, так как двум разным решениям $u_{1}(t), t \geqslant 0$, и $u_{2}(t), t \geqslant 0$, с одинаковыми начальными условиями $u_{1}(0)=u_{2}(0)=u_{0}$ соответствуют разные точки (траектории) $u_{1}(\cdot)$ и $u_{2}(\cdot)$ в фазовом пространстве $\mathscr{K}^{+}$.

Имея однозначную полугруппу $\{T(t)\}$, можно вновь обратиться к общей теории диссипативных полугрупп, изложенной в главе I. Наша цель - построить аттрактор полугруппы $\{T(t)\}$, действующей в фазовом пространстве $\mathscr{K}^{+}$. Притяжение к аттрактору семейств траекторий будет происходить в локальной топологии $\Theta_{+}^{\text {loc } п р о с т р а н с т в а ~} C^{1}\left(\mathbb{R}_{+} ; \mathbb{R}^{N}\right)$, которая определяется ниже.

В пространстве $C^{1}\left(\mathbb{R}_{+} ; \mathbb{R}^{N}\right)$ рассмотрим следующее семейство полунорм, зависящее от вещественного параметра $M \geqslant 0$ :

$$
\|y(\cdot)\|_{M}:=\|y(\cdot)\|_{C^{1}\left([0, M] ; \mathbb{R}^{N}\right)}=\max _{s \in[0, M]}|y(s)|+\max _{s \in[0, M]}\left|\partial_{t} y(s)\right| .
$$

Топологию $\Theta_{+}^{\text {loc }}$ в $C^{1}\left(\mathbb{R}_{+} ; \mathbb{R}^{N}\right)$ удобно задать с помощью определения сходимости последовательностей относительно полунорм (1.10).

ОПРЕДЕЛЕНИЕ 1.1. Последовательность $\left\{y_{m}(s), m \in \mathbb{N}\right\}$ из $C^{1}\left(\mathbb{R}_{+} ; \mathbb{R}^{N}\right)$ сходится при $m \rightarrow \infty$ к функции $y(s)$ в топологии $\Theta_{+}^{\text {loc }}$, если при любом $M \geqslant 0$

$$
\left\|y_{m}(\cdot)-y(\cdot)\right\|_{M} \rightarrow 0 \quad(m \rightarrow \infty) .
$$

Введенная топология локальной равномерной сходимости называется индуктивным пределом топологий $C^{1}\left([0, M] ; \mathbb{R}^{N}\right)$ при $M \rightarrow+\infty$.

ЗАмечание 1.1 . Топология $\Theta_{+}^{\text {loc }}$ в пространстве $C^{1}\left(\mathbb{R}_{+} ; \mathbb{R}^{N}\right)$ является метризуемой, а полученное метрическое пространство полно. Соответствующую метрика, которая называется метрикой Фреше, задается формулой

$$
\rho\left(y_{1}, y_{2}\right):=\sum_{n=1}^{\infty} 2^{-n} \frac{\left\|y_{1}-y_{2}\right\|_{n}}{1+\left\|y_{1}-y_{2}\right\|_{n}} .
$$

Мы будем рассматривать множества, компактные в топологии $\Theta_{+}^{\text {loc }}$. В связи с этим удобен следующий простой критерий компактности в $\Theta_{+}^{\text {loc }}$.

Лемма 1.1. Множество $K \subset C^{1}\left(\mathbb{R}_{+} ; \mathbb{R}^{N}\right)$ компактно в топологии $\Theta_{+}^{\text {loc }}$ тогда и только тогда, если $K$ компактно в $C^{1}\left([0, M] ; \mathbb{R}^{N}\right)$ при любом $M \geqslant 0$. 
УТВЕРЖДЕНИЕ 1.1. Пространство траекторий $\mathscr{K}^{+}$системы (1.1) замкнуто в топологии $\Theta_{+}^{\text {loc }}$.

Действительно, пусть $u_{m} \in \mathscr{K}^{+}$, причем $u_{m} \rightarrow u(m \rightarrow \infty)$ в $\Theta_{+}^{\text {loc }}$, т. е.

$$
\left|u_{m}(s)-u(s)\right|+\left|\partial_{t} u_{m}(s)-\partial_{t} u(s)\right| \rightarrow 0 \quad(m \rightarrow \infty)
$$

равномерно по $s \in[0, M]$ при каждом $M>0$. Тогда в уравнении

$$
\partial_{t} u_{m}(t)=-F\left(u_{m}(t)\right), \quad t \in[0, M]
$$

слева и справа можно перейти к пределу при $m \rightarrow \infty$ и получить равенство

$$
\partial_{t} u(t)=-F(u(t)), \quad t \in[0, M],
$$

при любом $M \geqslant 0$. Следовательно, $u(t), t \geqslant 0$, является решением системы (1.1), т. е. $u \in \mathscr{K}^{+}$, и множество $\mathscr{K}^{+}$замкнуто в топологии $\Theta_{+}^{\text {loc }}$.

Таким образом, имеется полное метрическое пространство $\mathscr{K}^{+}$, на котором действует полугруппа трансляций $\{T(t)\}$ (см. (1.9)).

Зафиксируем любое $t=h>0$. Пусть имеется произвольная последовательность $\left\{y_{m}\right\} \subset C^{1}\left(\mathbb{R}_{+} ; \mathbb{R}^{N}\right)$, причем $y_{m} \rightarrow y(m \rightarrow \infty)$ в топологии $\Theta_{+}^{\text {loc }}$, т. е. при любом $M \geqslant 0$ последовательность $y_{m}(s)$ сходится к $y(s)(m \rightarrow \infty)$ в пространстве $C^{1}\left([0, M] ; \mathbb{R}^{N}\right)$, а также в пространстве $C^{1}\left([0, h+M] ; \mathbb{R}^{N}\right)$ и, тем более, в $C^{1}\left([h, h+M] ; \mathbb{R}^{N}\right)$. Следовательно, $T(h) y_{m}(s) \rightarrow T(h) y(s)(m \rightarrow \infty)$ в $C^{1}\left([0, M] ; \mathbb{R}^{N}\right)$ при любом $M \geqslant 0$, т. е. $T(h) y_{m} \rightarrow T(h) y(m \rightarrow \infty)$ в $\Theta_{+}^{\text {loc }}$. Доказано следующее важное свойство полугруппы трансляций $\{T(t)\}$.

УтВеРЖДЕНИЕ 1.2. Полугруппа $\{T(t)\}$ непрерьвна в топологии $\Theta_{+}^{\text {loc }}$.

В общей теории диссипативных полугрупп изучается поведение множеств $T(t) \mathfrak{B}$ при $t \rightarrow+\infty$ в топологии $\Theta_{+}^{\text {loc }}$, где $\mathfrak{B}-$ произвольное ограниченное множество из $\mathscr{K}^{+}$. Возникает вопрос, в каком смысле следует определять ограниченность множеств в $\mathscr{K}^{+}$. Сразу заметим, что метрика (1.11) здесь не годится, так как, по этой метрике все пространство $\mathscr{K}^{+}$ограничено.

Для определения ограниченных множеств вводится другая полезная метрика. Рассмотрим следующее линейное подпространство:

$$
C_{\mathrm{b}}^{1}\left(\mathbb{R}_{+} ; \mathbb{R}^{N}\right) \subset C^{1}\left(\mathbb{R}_{+} ; \mathbb{R}^{N}\right)
$$

которое состоит из функций $y(\cdot)$, имеющих конечную норму

$$
\|y(\cdot)\|_{\mathrm{b}}:=\|y(\cdot)\|_{C_{\mathrm{b}}^{1}\left(\mathbb{R}_{+} ; \mathbb{R}^{N}\right)}=\sup _{s \geqslant 0}|y(s)|+\sup _{s \geqslant 0}\left|\partial_{t} y(s)\right| .
$$

Заметим, что $C_{\mathrm{b}}^{1}\left(\mathbb{R}_{+} ; \mathbb{R}^{N}\right)$ с нормой (1.12) является пространством Банаха. Кроме того, эта норма порождает топологию равномерной сходимости на всей полуоси $\mathbb{R}_{+}$, которая, очевидно, сильнее топологии локальной сходимости $\Theta_{+}^{\text {loс }}$

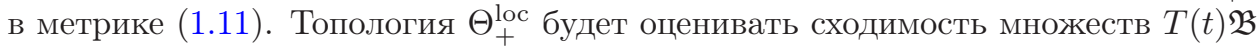
при $t \rightarrow+\infty$, а норма (1.12) будет использоваться для определения ограниченных множеств $\mathfrak{B}$ из $\mathscr{K}^{+}$. 
УтВеРЖДЕНИЕ 1.3. Пространство траекторий $\mathscr{K}^{+}$системы ОДУ (1.1) принадлежит пространству $C_{\mathrm{b}}^{1}\left(\mathbb{R}_{+} ; \mathbb{R}^{N}\right)$.

В самом деле, из неравенства (1.4) находим для любого $u \in \mathscr{K}^{+}$, что

$$
|u(s)|^{2} \leqslant|u(0)|^{2} \mathrm{e}^{-\delta s}+\frac{C}{\delta}, \quad \forall s \geqslant 0,
$$

и, следовательно, $u \in C_{\mathrm{b}}\left(\mathbb{R}_{+} ; \mathbb{R}^{N}\right)$. Напомним, что функция $F(u)$ непрерывна в $\mathbb{R}^{N}$, и, в частности, она ограничена на ограниченных в $\mathbb{R}^{N}$ множествах, т. е. $|F(u)|^{2} \leqslant C_{1}$ при $|u|^{2} \leqslant R$ для некоторой неубывающей функции $C_{1}=C_{1}(R)$. Тогда из уравнения (1.1) и из неравенства (1.13) находим, что

$$
\left|\partial_{t} u(s)\right|^{2} \leqslant C_{1}\left(|u(0)|^{2} \mathrm{e}^{-\delta s}+\frac{C}{\delta}\right), \quad \forall s \geqslant 0 .
$$

Следовательно, $\partial_{t} u \in C_{\mathrm{b}}\left(\mathbb{R}_{+} ; \mathbb{R}^{N}\right)$ и, тем самым, $\mathscr{K}^{+} \subset C_{\mathrm{b}}^{1}\left(\mathbb{R}_{+} ; \mathbb{R}^{N}\right)$.

Из неравенств (1.13) и (1.14) выводится еще одно важное свойство.

УтВЕРЖДЕНИЕ 1.4. Полугруппа $\{T(t)\}$ в пространстве $\mathscr{K}^{+}$имеет поглощающее множество

$$
\mathfrak{B}_{0}=\left\{\left.u \in C_{\mathrm{b}}^{1}\left(\mathbb{R}_{+} ; \mathbb{R}^{N}\right)|| u(s)\right|^{2} \leqslant \frac{2 C}{\delta},\left|\partial_{t} u(s)\right|^{2} \leqslant C_{1} \frac{2 C}{\delta}, \forall s \geqslant 0\right\} \cap \mathscr{K}^{+},
$$

т.е. для любого множества траекторий $\mathfrak{B} \subset \mathscr{K}^{+}$, ограниченного по норме пространства $C_{\mathrm{b}}^{1}\left(\mathbb{R}_{+} ; \mathbb{R}^{N}\right)$, найдется $t_{0}$ такое, что $T(t) \mathfrak{B} \subset \mathfrak{B}_{0}$ при $t \geqslant t_{0}$.

Отметим, что само множество $\mathfrak{B}_{0}$ инвариантно относительно $\{T(t)\}$, т. е.

$$
T(t) \mathfrak{B}_{0} \subseteq \mathfrak{B}_{0}, \quad \forall t \geqslant 0 .
$$

Сформулируем еще одно ключевое свойство поглощающего множества $\mathfrak{B}_{0}$.

УТВеРЖДЕНИЕ 1.5. Множество $\mathfrak{B}_{0}$ компактно в топологии $\Theta_{+}^{\text {loc }}$.

Докажем это. В силу леммы 1.1 достаточно проверить, что $\mathfrak{B}_{0}$ компактно в пространстве $C^{1}\left([0, M] ; \mathbb{R}^{N}\right)$ при любом $M \geqslant 0$. Любая последовательность $\left\{u_{m}\right\} \subset \mathfrak{B}_{0}$ равномерно ограничена в $C^{1}\left([0, M] ; \mathbb{R}^{N}\right)$. Тогда в силу компактности вложения: $C^{1}\left([0, M] ; \mathbb{R}^{N}\right) \Subset C\left([0, M] ; \mathbb{R}^{N}\right)$, найдется сходящаяся подпоследовательность $u_{m_{i}}(s) \rightarrow u(s)\left(m_{i} \rightarrow \infty\right)$ в $C\left([0, M] ; \mathbb{R}^{N}\right)$. Функции $u_{m_{i}}(t)$ удовлетворяют ОДУ

$$
\partial_{t} u_{m_{i}}(t)=-F\left(u_{m_{i}}(t)\right), \quad t \in[0, M] .
$$

Функция $F(u)$ непрерывна, следовательно, $F\left(u_{m_{i}}(t)\right) \rightarrow F(u(t))\left(m_{i} \rightarrow \infty\right)$ в $C\left([0, M] ; \mathbb{R}^{N}\right)$. Поэтому последовательность $\left\{\partial_{t} u_{m_{i}}(t)\right\}$ также является сходящейся в $C\left([0, M] ; \mathbb{R}^{N}\right)$. В итоге последовательность $\left\{u_{m_{i}}\right\}$ сходится в пространстве $C^{1}\left([0, M] ; \mathbb{R}^{N}\right)$. Кроме того, множество $\mathfrak{B}_{0}$ замкнуто в $C^{1}\left([0, M] ; \mathbb{R}^{N}\right)$, так как $\mathscr{K}^{+}$замкнуто в $\Theta_{+}^{\text {loc }}$ (см. утверждение 1.1$)$. Наконец, заключаем, что $\mathfrak{B}_{0}$ компактно в топологии $\Theta_{+}^{\text {loc }}$.

Теперь все готово для изучения аттрактора полугруппы $\{T(t)\}$ в пространстве траекторий $\mathscr{K}^{+}$. 
Построение траекторного аттрактора. В утверждении 1.4 было построено поглощающее множество полугруппы $\{T(t)\}$. Напомним определение притягивающего множества, применительно к этой полугруппе (см. определение 1.1 главы I).

ОПРЕДЕлЕниЕ 1.2. Множество $\mathscr{P} \subseteq \mathscr{K}^{+}$называют притягивающим (в топологии $\Theta_{+}^{\text {loc }}$ ) для полугруппы $\{T(t)\}$, если для любого множества $\mathfrak{B} \subset \mathscr{K}^{+}$, ограниченного в $C_{\mathrm{b}}^{1}\left(\mathbb{R}_{+} ; \mathbb{R}^{N}\right)$, множество $T(t) \mathfrak{B}$ сходится к $\mathscr{P}(t \rightarrow+\infty)$ в топологии $\Theta_{+}^{\text {loc }}$, т. е. для любого $M \geqslant 0$

$$
\operatorname{dist}_{C^{1}\left([0, M] ; \mathbb{R}^{N}\right)}(T(t) \mathfrak{B}, \mathscr{P}) \rightarrow 0 \quad(t \rightarrow+\infty) .
$$

Дадим определение траекторного аттрактора полугруппы $\{T(t)\}$, действующей в пространстве траекторий $\mathscr{K}^{+}$уравнения (1.1).

ОПредЕЛЕниЕ 1.3. Множество $\mathfrak{A} \subseteq \mathscr{K}^{+}$называется траекторным атmрактором полугруппы $\{T(t)\}$ на $\mathscr{K}^{+}$, если оно ограничено в $C_{\mathrm{b}}^{1}\left(\mathbb{R}_{+} ; \mathbb{R}^{N}\right)$, компактно в топологии $\Theta_{+}^{\text {loc }}$, является притягивающим множеством полугруппы $\{T(t)\}$ на $\mathscr{K}^{+}$в топологии $\Theta_{+}^{\text {loc }}$ и строго инвариантно относительно $\{T(t)\}$, т. е. $T(t) \mathfrak{A}=\mathfrak{A}$ для всех $t \geqslant 0$.

Легко видеть, что траекторный аттрактор $\mathfrak{A}$ полугруппы $\{T(t)\}$ определяется однозначно. Множество $\mathfrak{A}$ также называется траекторным аттрактором системы ОДУ (1.1).

Теорема 1.1. Система ОДУ (1.1), удовлетворяющая условию диссипативности (1.3), имеет траекторньй аттрактор $\mathfrak{A}$.

Мы получим траекторный аттрактор, исходя из поглощающего множества $\mathfrak{B}_{0}$, построенного в утверждении 1.4. Рассмотрим действие полугруппы $\{T(t)\}$ на инвариантном множестве $\mathfrak{B}_{0}$ (см. (1.15)), которое является компактным метрическим пространством (в топологии $\Theta_{+}^{\text {loc }}$, см. утверждение 1.5). Полугруппа $\{T(t)\}$ непрерывна в этой топологии (см. утверждение 1.2). Поэтому применима общая теорема 1.1 главы I о существовании глобального аттрактора полугруппы в полном метрическом пространстве. Отметим, что само пространство $\mathfrak{B}_{0}$ является компактным поглощающим множеством для любого своего (ограниченного) подмножества $\mathfrak{B} \subseteq \mathfrak{B}_{0}$. Значит, полугруппа $\left.\{T(t)\}\right|_{\mathfrak{B}_{0}}$ имеет глобальный аттрактор, который мы обозначим $\mathfrak{A}$, причем

$$
\mathfrak{A}=\omega\left(\mathfrak{B}_{0}\right)=\bigcap_{t \geqslant 0} T(t) \mathfrak{B}_{0}
$$

(см. замечание 1.1 главы I). При этом множество $\mathfrak{A}$ является компактным в $\Theta_{+}^{\text {loc }}$, ограниченным в $C_{\mathrm{b}}^{1}\left(\mathbb{R}_{+} ; \mathbb{R}^{N}\right)$ и строго инвариантным относительно полугруппы $\{T(t)\}$. Покажем, что множество $\mathfrak{A}$ притягивает любые ограниченные (в $\left.C_{\mathrm{b}}^{1}\left(\mathbb{R}_{+} ; \mathbb{R}^{N}\right)\right)$ множества $\mathfrak{B} \subset \mathscr{K}^{+}$. В самом деле, если $\mathfrak{B} \subseteq \mathfrak{B}_{0}$, то, как уже установлено, $T(t) \mathfrak{B} \rightarrow \mathfrak{A}$ при $t \rightarrow+\infty$. Если $\mathfrak{B} \nsubseteq \mathfrak{B}_{0}$, то из утверждения 1.4 следует, что $T\left(t_{1}\right) \mathfrak{B} \subseteq \mathfrak{B}_{0}$ при достаточно большом $t_{1}=t_{1}(\mathfrak{B})$. Следовательно,

$$
T(t) \mathfrak{B}=T\left(t-t_{1}+t_{1}\right) \mathfrak{B}=T\left(t-t_{1}\right) T\left(t_{1}\right) \mathfrak{B} \subseteq T\left(t-t_{1}\right) \mathfrak{B}_{0} \rightarrow \mathfrak{A} \quad(t \rightarrow+\infty) .
$$

Мы проверили, что множество $\mathfrak{A}$ удовлетворяет всем требованиям, предъявляемым к траекторному аттрактору, и теорема 1.1 доказана. 
Ядро системы ОДУ. Для описания структуры построенного выше траекторного аттрактора мы воспользуемся понятием ядра полугруппы или дифференциального уравнения, которое уже использовалось в главе I для описания структуры глобального аттрактора.

По определению, функция $u(\cdot) \in C^{1}\left(\mathbb{R} ; \mathbb{R}^{N}\right)$ называется полной траекторией уравнения (1.1), если она является решением этого уравнения на всей оси времени $-\infty<t<+\infty$ :

$$
\partial_{t} u(t)=-F(u(t)), \quad \forall t \in \mathbb{R}
$$

Полная траектория называется ограниченной, если

$$
|u(t)| \leqslant C_{u}, \quad|\partial u(t)| \leqslant C_{u}, \quad \forall t \in \mathbb{R}
$$

т. е. когда $u \in C_{\mathrm{b}}^{1}\left(\mathbb{R} ; \mathbb{R}^{N}\right)$. Семейство всех ограниченных полных траекторий уравнения (1.1) называется ядром этого уравнения, которое обозначается

$$
\mathscr{K}:=\left\{u(\cdot) \in C_{\mathrm{b}}^{1}\left(\mathbb{R} ; \mathbb{R}^{N}\right) \mid u(\cdot) \text { удовлетворяет }(1.16)\right\} .
$$

Через $\Pi_{+} f$ обозначается сужение на полуось $\mathbb{R}_{+}$функции $f$, заданной на всей оси $\mathbb{R}$.

ТеОРема 1.2. При выполнении условий теоремы 1.1

$$
\mathfrak{A}=\Pi_{+} \mathscr{K},
$$

причем ядро $\mathscr{K}$ системь ОДУ (1.1) ограничено в $C_{\mathrm{b}}^{1}\left(\mathbb{R} ; \mathbb{R}^{N}\right)$ и компактно $8 \Theta^{\mathrm{loc}}$.

Топология $\Theta^{\text {loc } о п р е д е л я е т с я ~ в ~ п р о с т р а н с т в е ~} C^{1}\left(\mathbb{R} ; \mathbb{R}^{N}\right)$ аналогично $\Theta_{+}^{\text {loc }}$ как индуктивный предел топологий $C^{1}\left([-M, M] ; \mathbb{R}^{N}\right)$ при $M \rightarrow+\infty$, а метрика в пространстве $C_{\mathrm{b}}^{1}\left(\mathbb{R} ; \mathbb{R}^{N}\right)$ задается аналогично $(1.12)$, но теперь в этой формуле супремум следует брать по $s \in \mathbb{R}$.

Доказательство теоремы 1.2 основано на свойстве строгой инвариантности траекторного аттрактора; мы его опускаем (см. доказательство теоремы 2.2, а также [12]).

Из неравенств (1.13) и (1.14) выводится следующее полезное свойство ядра.

СлеДСТвиЕ 1.1. Для любого $u \in \mathscr{K}$ справедливы неравенства

$$
|u(t)|^{2} \leqslant \frac{C}{\delta}, \quad\left|\partial_{t} u(t)\right|^{2} \leqslant C_{1} \frac{C}{\delta}, \quad \forall t \in \mathbb{R} .
$$

Завершая рассмотрение обыкновенных дифференциальных уравнений, мы для системы (1.1) с помощью ее ядра и траекторного аттрактора построим некоторое множество в пространстве $\mathbb{R}^{N}$, которое является обобщением глобального аттрактора системы ОДУ, если для нее справедлива теорема единственности решения задачи Коши. 
Глобальный аттрактор системы ОДУ без единственности. Рассмотрим пространство траекторий $\mathscr{K}^{+} \subseteq C_{\mathrm{b}}^{1}\left(\mathbb{R}_{+} ; \mathbb{R}^{N}\right)$, а в нем произвольное ограниченное множество $\mathfrak{B} \subset \mathscr{K}^{+}$. Обозначим через $\mathfrak{B}(t)$ сечение множества $\mathfrak{B}$ в момент времени $t$ :

$$
\mathfrak{B}(t):=\{u(t) \mid u(\cdot) \in \mathfrak{B}\}, \quad t \geqslant 0 .
$$

Ясно, что $\mathfrak{B}(t) \subset \mathbb{R}^{N}$, и сечения равномерно (по $t \geqslant 0$ ) ограничены в $\mathbb{R}^{N}$, так как

$$
|\mathfrak{B}(t)|:=\sup _{y \in \mathfrak{B}(t)}|y| \leqslant\|\mathfrak{B}\|_{C_{\mathrm{b}}\left(\mathbb{R}_{+} ; \mathbb{R}^{N}\right)}:=\sup _{u \in \mathfrak{B}}\|u(\cdot)\|_{C_{\mathrm{b}}\left(\mathbb{R}_{+} ; \mathbb{R}^{N}\right)} \leqslant C_{\mathfrak{B}}, \quad \forall t \geqslant 0 .
$$

Пусть $\mathfrak{A}$ - траекторный аттрактор системы (1.1), построенный в теореме 1.1. Рассмотрим сечение множества $\mathfrak{A}$ в момент времени $t \geqslant 0$ :

$$
\mathfrak{A}(t):=\{u(t) \mid u \in \mathfrak{A}\} \subset \mathbb{R}^{N} .
$$

Напомним, что $\mathfrak{A}$ строго инвариантно под действием полугруппы трансляций, поэтому

$$
\mathfrak{A}(t)=\mathfrak{A}(0), \quad \forall t \geqslant 0 .
$$

Кроме того, множество $\mathfrak{A}$ ограничено в $C_{\mathrm{b}}^{1}\left(\mathbb{R}_{+} ; \mathbb{R}^{N}\right)$ и компактно в $\Theta_{+}^{\text {loc }}$. Следовательно, его сечение $\mathfrak{A}(0)$ ограничено и компактно в $\mathbb{R}^{N}$. Наконец, $\mathfrak{A}$ притягивает любое ограниченное множество $\mathfrak{B}$, т. е.

$$
\operatorname{dist}_{C^{1}\left([0, M] ; \mathbb{R}^{N}\right)}(T(t) \mathfrak{B}, \mathfrak{A}) \rightarrow 0 \quad(t \rightarrow+\infty)
$$

для любого $M \geqslant 0$, и, в частности, при $M=0$

$$
\operatorname{dist}_{\mathbb{R}^{N}}(\mathfrak{B}(t), \mathfrak{A}(0)) \rightarrow 0 \quad(t \rightarrow+\infty),
$$

так как очевидно, что $(T(t) \mathfrak{B})(0)=\mathfrak{B}(t)$.

Полученные свойства сечения $\mathfrak{A}(0)$ приводят к следующему определению.

ОПредЕление 1.4. Множество $\mathscr{A} \subset \mathbb{R}^{N}$ называется глобалъным аттрактором системы (1.1), если оно ограничено и замкнуто в $\mathbb{R}^{N}$, а кроме того, выполнены следующие свойства:

1) для любого множества $\mathfrak{B} \subset \mathscr{K}^{+}$, ограниченного по норме $C_{\mathrm{b}}^{1}\left(\mathbb{R}_{+} ; \mathbb{R}^{N}\right)$, его сечение $\mathfrak{B}(t)$ притягивается к $\mathscr{A}$ при $t \rightarrow+\infty$, т. е.

$$
\operatorname{dist}_{\mathbb{R}^{N}}(\mathfrak{B}(t), \mathscr{A}) \rightarrow 0 \quad(t \rightarrow+\infty)
$$

2) $\mathscr{A}$ является минимальным ограниченным и замкнутым множеством в пространстве $\mathbb{R}^{N}$, которое притягивает при $t \rightarrow+\infty$ сечения любого ограниченного множества из $\mathscr{K}^{+}$.

Установив свойства (1.19) и (1.20), мы доказали следующий результат.

Теорема 1.3. При выполнении условий теоремы 1.1 множество $\mathscr{A}:=$ $\mathfrak{A}(0)=\mathscr{K}(0)$ является глобальным аттрактором системы ОДУ (1.1) в смысле определения 1.4 . 
ЗАмЕчаниЕ 1.2. Если дополнительно известно, что функция $F(u)$ в системе ОДУ (1.1) является липшицевой (т. е. выполнено (1.5)), то для однозначно разрешимой задачи Коши (1.1)-(1.2) по обычной схеме строится полугруппа $\{S(t)\}$ в $\mathbb{R}^{N}$ (см. (1.7)), которая имеет (обычный) глобальный аттрактор $\mathscr{A}=\mathscr{K}(0)$, причем в соотношении $(1.21) \mathfrak{B}(t)=S(t) B$, где $B=\mathfrak{B}(0)-$ произвольное ограниченное в $\mathbb{R}^{N}$ множество начальных данных. Очевидно, что это множество $\mathscr{A}$ также совпадает $\mathfrak{A}(0)$. Кроме того, имея глобальный аттрактор $\mathscr{A}$ задачи (1.1)-(1.2), траекторный аттрактор системы (1.1) можно построить по формуле

$$
\mathfrak{A}=\left\{u(t)=S(t) u_{0}, t \geqslant 0 \mid u_{0} \in \mathscr{A}\right\} .
$$

В этом разделе на относительно простом модельном примере системы ОДУ был продемонстрирован метод траекторных аттракторов, позволяющий исследовать поведение решений эволюционных уравнений, для которых может не выполняться теорема единственности решения соответствующей задачи Коши. В следующих разделах этот подход будет применяться к существенно более сложным уравнениям математической физики.

\section{2. Траекторный аттрактор общей системы реакции-диффузии}

Общая РД-система и ее слабые решения. Рассматривается система дифференциальных уравнений следующего вида:

$$
\partial_{t} u=\mathbf{a} \Delta u-f(u)+g(x),\left.\quad u\right|_{\partial \Omega}=0, \quad x \in \Omega \Subset \mathbb{R}^{n}, \quad t \geqslant 0 .
$$

Здесь $u=\left(u^{1}(x, t), \ldots, u^{N}(x, t)\right)$ - неизвестная вектор-функция, $g(x)=\left(g^{1}(x)\right.$, $\left.\ldots, g^{N}(x)\right)$ - внешнее воздействие и $f(u)=\left(f^{1}(u), \ldots, f^{N}(u)\right)$ - нелинейная функция взаимодействия. Вещественная $(N \times N)$-матрица а имеет положительную симметричную часть $\left(\mathbf{a}+\mathbf{a}^{*}\right) / 2 \geqslant \beta \mathbf{I}, \beta>0$.

ЗАмечАниЕ 2.1. Частным случаем РД-системы (2.1) является двумерная система (I.2.1)-(I.2.3), в которой матрица а является диагональной, а внешнее воздействие $g$ тождественно равно нулю. Другой пример - это комплексное уравнение Гинзбурга-Ландау, записанное в векторной форме (I.2.37), в которой матрица а является не диагональной, а кососимметрической.

Вводятся пространства

$$
H:=\left[L_{2}(\Omega)\right]^{N} \quad \text { и } \quad V:=\left[H_{0}^{1}(\Omega)\right]^{N} .
$$

Нормы в этих пространствах обозначаются

$$
|v|^{2}:=\int_{\Omega} \sum_{i=1}^{N}\left|v^{i}(x)\right|^{2} d x \quad \text { и } \quad\|v\|^{2}:=\int_{\Omega} \sum_{i=1}^{N}\left|\nabla v^{i}(x)\right|^{2} d x .
$$

Кроме того, $V^{\prime}=\left[H^{-1}(\Omega)\right]^{N}$ обозначает пространство, сопряженное к $V$. 
Предполагается, что $g \in V^{\prime}$, а функция взаимодействия $f(\cdot)$ принадлежит $C\left(\mathbb{R}^{N} ; \mathbb{R}^{N}\right)$ и удовлетворяет неравенствам

$$
\begin{aligned}
& \sum_{i=1}^{N}\left|f^{i}(v)\right|^{\frac{p_{i}}{p_{i}-1}} \leqslant C_{2}\left(\sum_{i=1}^{N}\left|v_{i}\right|^{p_{i}}+1\right), \\
& \sum_{i=1}^{N} \gamma_{i}\left|v_{i}\right|^{p_{i}}-C_{1} \leqslant \sum_{i=1}^{N} f^{i}(v) v_{i}, \quad \forall v \in \mathbb{R}^{N},
\end{aligned}
$$

где $\gamma_{i}>0, i=1, \ldots, N$. Для определенности считается, что $2 \leqslant p_{1} \leqslant \cdots \leqslant p_{N}$. Условие (2.2) связано с тем, что в реальных РД-системах функции $f^{i}(u)$ являются многочленами. Неравенство (2.3) отражает важное условие диссипативности.

Обозначим $q_{i}=p_{i} /\left(p_{i}-1\right), 1 / p_{i}+1 / q_{i}=1,1<q_{i} \leqslant 2, i=1, \ldots, N$. Вводятся также векторные обозначения $\mathbf{p}=\left(p_{1}, \ldots, p_{N}\right), \mathbf{q}=\left(q_{1}, \ldots, q_{N}\right)$ и следующие пространства:

$$
\begin{aligned}
L_{\mathbf{p}}(\Omega) & :=L_{p_{1}}(\Omega) \times \cdots \times L_{p_{N}}(\Omega), \\
L_{\mathbf{p}}\left(0, M ; L_{\mathbf{p}}(\Omega)\right) & :=L_{p_{1}}\left(0, M ; L_{p_{1}}(\Omega)\right) \times \cdots \times L_{p_{N}}\left(0, M ; L_{p_{N}}(\Omega)\right) .
\end{aligned}
$$

Введем также банахово пространство

$$
\mathscr{L}(0, M):=\mathbf{L}_{2}\left(0, M ; \mathbf{V}^{\prime}\right)+\mathbf{L}_{\mathbf{q}}\left(0, M ; \mathbf{L}_{\mathbf{q}}(\Omega)\right)
$$

Если $u(x, t) \in L_{\mathbf{p}}\left(0, M ; L_{\mathbf{p}}(\Omega)\right)$, то из $(2.2)$ следует, что $f(u(x, t)) \in L_{\mathbf{q}}(0, M$; $\left.L_{\mathbf{q}}(\Omega)\right)$, причем

$$
\sum_{i=1}^{N}\left\|f_{i}(u(\cdot))\right\|_{L_{q_{i}}\left(0, M ; L_{q_{i}}\right)}^{q_{i}} \leqslant C_{2}\left(\sum_{i=1}^{N}\|u(\cdot)\|_{L_{p_{i}}\left(0, M ; L_{p_{i}}\right)}^{p_{i}}+M|\Omega|\right) .
$$

Кроме того, если $u(x, t) \in L_{2}(0, M ; V)$, то $\mathbf{a} \Delta u(x, t)+g(x) \in L_{2}\left(0, M ; V^{\prime}\right)$.

Функция $u(x, t), x \in \Omega, t \geqslant 0$, называется (2.1), если

$$
u(x, t) \in \mathbf{L}_{\mathbf{p}}\left(0, M ; \mathbf{L}_{\mathbf{p}}(\Omega)\right) \cap \mathbf{L}_{2}(0, M ; \mathbf{V})
$$

при каждом $M>0$, и при любой (пробной) функции $\varphi(x) \in \mathbf{L}_{\mathbf{p}}(\Omega) \cap \mathbf{V}$ выполнено (в обобщенном смысле) тождество

$$
\frac{d}{d t} \int_{\Omega} u(x, t) \cdot \varphi(x) d x+\int_{\Omega}\{\mathbf{a} \nabla u(x, t) \cdot \nabla \varphi(x)+f(u(x, t)) \cdot \varphi(x)\} d x=\langle g, \varphi\rangle .
$$

При этом из уравнения (2.1) следует, что

$$
\partial_{t} u(x, t) \in \mathscr{L}(0, M):=\mathbf{L}_{2}\left(0, M ; \mathbf{V}^{\prime}\right)+\mathbf{L}_{\mathbf{q}}\left(0, M ; \mathbf{L}_{\mathbf{q}}(\Omega)\right) .
$$

По теореме вложения Соболева $\mathscr{L}(0, M) \subset \mathbf{L}_{\mathbf{q}}\left(0, M ; \mathbf{H}^{-\mathbf{r}}(\Omega)\right)$, где $\mathbf{H}^{-\mathbf{r}}(\Omega)=$ $H^{-r_{1}}(\Omega) \times \cdots \times H^{-r_{N}}(\Omega), \mathbf{r}=\left(r_{1}, \ldots, r_{N}\right)$ и

$$
r_{i} \equiv \max \left\{1, n\left(\frac{1}{q_{i}}-\frac{1}{2}\right)\right\}, \quad i=1, \ldots, N .
$$


Следовательно, для любого слабого решения (2.1) выполнено включение

$$
\partial_{t} u(x, t) \in \mathbf{L}_{\mathbf{q}}\left(0, M ; \mathbf{H}^{-\mathbf{r}}(\Omega)\right),
$$

и, в частности, $u(x, t)$ удовлетворяет $(2.1)$ в смысле пространства обобщенных функций $D^{\prime}\left(0, M ; \mathbf{H}^{-\mathbf{r}}(\Omega)\right)$ для любого $M>0$.

Мы будем в дальнейшем (для упрощения записи) иногда опускать пространственный аргумент $x$ в решениях системы $(2.1)$ и писать $u(t)$ вместо $u(x, t)$.

Нам потребуется следующий классический результат из [47] (см. также [26]).

Лемма 2.1 (Лионс-Мадженес). Пусть заданъ пространства Банаха Е $u E_{0}$, причем $E \subset E_{0}$ и это вложение непрерьвно. Пусть имеется функиия $\phi(t), t \in[0, M], \phi(\cdot) \in L_{\infty}(0, M ; E)$, которая является слабо непрерьвной со значениям в $E_{0}$, m.е. $\phi(t) \in E_{0}$ при всех $t \in[0, M]$ и при любом $\xi \in E_{0}^{*}$ скалярная функиия $\langle\phi(t), \xi\rangle$ непрерывна по $t$. Тогда $\phi(\cdot)$ слабо непрерывна со значениями в $E$, кроме того,

$$
\|\phi(t)\|_{E} \leqslant\|\phi(\cdot)\|_{L_{\infty}(0, M ; E)}, \quad \forall t \in[0, M] .
$$

Если $u(t)$ является слабым решением системы $(2.1)$, то, очевидно, $u(\cdot) \in$ $C\left([0, M] ; \mathbf{H}^{-\mathbf{r}}(\Omega)\right)$. Кроме того, если известно, что $u(\cdot) \in L_{\infty}(0, M ; H)$, то при $\phi(\cdot)=u(\cdot), E=H$ и $E_{0}=\mathbf{H}^{-\mathbf{r}}(\Omega)$ из леммы Лионса-Мадженеса вытекает, что $u(\cdot) \in C_{\mathrm{w}}([0, M] ; H)$. Поэтому для уравнений $(2.1)$ можно задать начальные условия

$$
\left.u\right|_{t=0}=u_{0}, \quad \text { где } u_{0} \in H .
$$

Приведем известный результат о существовании слабого решения системы (2.1), который доказывается по методу Галёркина (см. [12]).

УтвеРЖДЕНИЕ 2.1. Пусть $g(\cdot) \in V^{\prime}$, функция $f(v)$ удовлетворяет неравенствам (2.2), (2.3) и начальное условие и принадлежит Н. Тогда РД-система (2.1) имеет глобальное слабое решение

$$
u(t) \in L_{\mathbf{p}}^{\text {loc }}\left(\mathbb{R}_{+} ; L_{\mathbf{p}}(\Omega)\right) \cap L_{2}^{\text {loc }}\left(\mathbb{R}_{+} ; V\right) \cap L_{\infty}\left(\mathbb{R}_{+} ; H\right),
$$

которое удовлетворяет начальному условию $и(0)=u_{0}$.

ЗАмЕчАниЕ 2.2. При сформулированных условиях слабое решение задачи может быть не единственным, так как вектор-функция $f(u)$ не предполагается липшицевой. Теорему единственности можно доказать, если известно, что $f(u) \in C^{1}\left(\mathbb{R}^{N} ; \mathbb{R}^{N}\right)$, причем якобиан $\mathbf{J}(u)=\partial f(u) / \partial u$ удовлетворяет неравенству вида (I.2.14) для некоторого числа $D>0$. (Например для уравнения Гинзбурга-Ландау (I.2.35) это неравенство выполнено при $|\beta| \leqslant \sqrt{3}$.) Однако это условие представляется весьма ограничительным, поэтому оно не предполагается при построении траекторного аттрактора системы (2.1).

В [12] также установлено, что любое слабое решение $u(t) \in L_{\mathbf{p}}\left(0, M ; L_{\mathbf{p}}(\Omega)\right) \cap$ $L_{2}(0, M ; V)$ РД-системы $(2.1)$ является сильно непрерывной функцией в пространстве $C([0, M] ; H)$, причем функция $|u(t)|^{2}$ является абсолютно непрерывной по $t \in[0, M]$ и выполнено следующее дифференциальное тождество:

$$
\frac{1}{2} \frac{d}{d t}|u(t)|^{2}+(\mathbf{a} \nabla u(t), \nabla u(t))+(f(u(t)), u(t))=\langle g, u(t)\rangle
$$


при почти всех $t \in[0, M]$. Это соотношение называется энергетическим равенством для РД-системы.

Из (2.7) выводится, что любое слабое решение $u(t)$ РД-системы (2.1) удовлетворяет неравенствам

$$
\begin{gathered}
|u(t)|^{2} \leqslant|u(0)|^{2} \mathrm{e}^{-\lambda_{1} \beta t}+R_{1}^{2}, \\
\beta \int_{t}^{t+1}\|u(s)\|^{2} d s+2 \sum_{i=1}^{N} \gamma_{i} \int_{t}^{t+1}\left\|u_{i}(s)\right\|_{L_{p_{i}}}^{p_{i}} d s \leqslant|u(t)|^{2}+R_{2}^{2},
\end{gathered}
$$

где $\lambda_{1}$ - первое собственное значение скалярного оператора $-\Delta$ с граничными условиями Дирихле. Величины $R_{1}$ и $R_{2}$ зависят от $\|g\|_{V^{\prime}}$ и не зависят от $u(0)$.

Для построения траекторного аттрактора системы (2.1) необходимо задать пространство траекторий $\mathscr{K}^{+}$. Для этого можно поступить аналогично тому, как это было сделано при исследовании системы ОДУ $(1.1)$, и объединить в $\mathscr{K}^{+}$ все глобальные слабые решения (2.1).

ОПРЕДЕЛЕНИЕ 2.1. Пространством траекторий $\mathscr{K}+$ РД-системы (2.1) называется совокупность всех ее слабых решений $u(t)$ из класса

$$
L_{\mathbf{p}}^{\text {loc }}\left(\mathbb{R}_{+} ; L_{\mathbf{p}}(\Omega)\right) \cap L_{2}^{\text {loc }}\left(\mathbb{R}_{+} ; V\right) \cap L_{\infty}\left(\mathbb{R}_{+} ; H\right) .
$$

Из утверждения 2.1 вытекает, что для любого $u_{0} \in H$ существует траектория $u(\cdot) \in \mathscr{K}^{+}$такая, что $u(0)=u_{0}$. Следовательно, у системы (2.1) имеется достаточно широкое пространство траекторий.

Если $u(\cdot) \in \mathscr{K}^{+}$, то из уравнения (2.1) получаем, что

$$
\partial_{t} u(\cdot) \in \mathscr{L}^{\text {loc }}\left(\mathbb{R}_{+}\right):=\mathbf{L}_{2}^{\text {loc }}\left(\mathbb{R}_{+} ; \mathbf{V}^{\prime}\right)+\mathbf{L}_{\mathbf{q}}^{\text {loc }}\left(\mathbb{R}_{+} ; \mathbf{L}_{\mathbf{q}}(\Omega)\right) .
$$

Построение траекторного аттрактора. Пространство траекторий $\mathscr{K}^{+}$ системы (2.1) является подмножеством линейного пространства

$$
\mathscr{F}_{+}^{\text {loc }}:=L_{\infty}^{\text {loc }}\left(\mathbb{R}_{+} ; H\right) \cap L_{2}^{\text {loc }}\left(\mathbb{R}_{+} ; V\right) \cap L_{\mathbf{p}}^{\text {loc }}\left(\mathbb{R}_{+} ; L_{\mathbf{p}}(\Omega)\right) \cap\left\{\partial_{t} v \in \mathscr{L}^{\text {loc }}\left(\mathbb{R}_{+}\right)\right\}
$$

Напомним, что линейное пространство $L_{p}^{\text {loc }}\left(\mathbb{R}_{+} ; X\right)$, где $p \geqslant 1$ и $X$ - некоторое банахово пространство, состоит из измеримых функций $v(t), t \in \mathbb{R}_{+}$, со значениями в $X$, интегрируемых по Бохнеру и для которых конечен интеграл

$$
\int_{0}^{M}\|v(s)\|_{X}^{p} d s, \quad \forall M>0 .
$$

В пространстве $L_{p}^{\text {loc }}\left(\mathbb{R}_{+} ; X\right)$ выделяется линейное нормированное подпространство $L_{p}^{\mathrm{b}}\left(\mathbb{R}_{+} ; X\right)$ функций, имеющих конечную норму

$$
\|v(\cdot)\|_{L_{p}^{\mathrm{b}}\left(\mathbb{R}_{+} ; X\right)}:=\left[\sup _{t \in \mathbb{R}_{+}} \int_{t}^{t+1}\|v(s)\|_{X}^{p} d s\right]^{1 / p} .
$$

Пространство $L_{p}^{\text {loc }}\left(\mathbb{R}_{+} ; X\right)$, очевидно, является банаховым.

Введем банахово пространство $\mathscr{F}_{+}^{\mathrm{b}} \subset \mathscr{F}_{+}^{\text {loc: }}$

$$
\mathscr{F}_{+}^{\mathrm{b}}:=L_{\infty}\left(\mathbb{R}_{+} ; H\right) \cap L_{2}^{\mathrm{b}}\left(\mathbb{R}_{+} ; V\right) \cap L_{\mathbf{p}}^{\mathrm{b}}\left(\mathbb{R}_{+} ; L_{\mathbf{p}}(\Omega)\right) \cap\left\{\partial_{t} v \in \mathscr{L}^{\mathrm{b}}\left(\mathbb{R}_{+}\right)\right\} .
$$


Норма в $\mathscr{F}_{+}^{\mathrm{b}}$ задается формулой

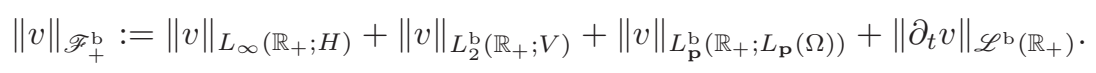

Следуя схеме раздела 1, для построения траекторного аттрактора в пространстве траекторий $\mathscr{K}^{+}$уравнения $(2.1)$ необходимо ввести топологию $\Theta_{+}^{\mathrm{w}, \text { loc }}$ в $\mathscr{F}_{+}^{\text {loc }}$. Проще всего построить траекторный аттрактор в “слабой” топологии.

Зададим топологию $\Theta_{+}^{\mathrm{w}, \mathrm{loc}}$, определив сходимость последовательностей. По определению, последовательность функций $\left\{v_{m}(t)\right\} \subset \mathscr{F}_{+}^{\text {loc }}$ сходится при $m \rightarrow \infty$ к функции $v(t) \in \mathscr{F}_{+}^{\text {loc }}$ в топологии $\Theta_{+}^{\mathrm{w}, \text { loc }}$, если для любого $M>0$ имеют место следующие сходимости при $m \rightarrow \infty$ :

$$
\left\{\begin{array}{lc}
v_{m}(\cdot) \rightarrow v_{m}(\cdot) & \text { слабо в } L_{2}(0, M ; V), \text { слабо в } L_{\mathbf{p}}\left(0, M ; L_{\mathbf{p}}(\Omega)\right) \\
\partial_{t} v_{m}(\cdot) \rightarrow \partial_{t} v_{m}(\cdot) & \text { слабо в } \mathscr{L}(0, M) .
\end{array}\right.
$$

Аналогичным образом в пространстве $\mathscr{F}_{+}^{\text {loc }}$ задается топология $\Theta_{+}^{\text {s,loc }}$ cuльной локальной сходимости, которая порождается сходимостью последовательностей $\left\{v_{m}(\cdot)\right\}$ и $\left\{\partial_{t} v_{m}(\cdot)\right\}$ по норме в тех же самых пространствах при каждом $M>0$. Легко проверить, что топология $\Theta_{+}^{\text {s,loc }}$ метризуема, а соответствующее метрическое пространство $\mathscr{F}_{+}^{\text {s,loc }}=\mathscr{F}_{+}^{\text {loc }} \cap \Theta_{+}^{\text {s,loc }}$ является полным. Очевидно, что топология $\Theta_{+}^{\text {s,loc }}$ сильнее, чем $\Theta_{+}^{\mathrm{w}, \text { loc }}$.

При исследовании траекторного аттрактора РД-системы (2.1) мы будем использовать обе топологии: слабую $\Theta_{+}^{\mathrm{w}, \mathrm{loc}}$ и сильную $\Theta_{+}^{\mathrm{s}, \mathrm{loc}}$.

ЗАМЕЧАНИЕ 2.3. Любой шар

$$
\mathscr{B}_{r}=\left\{v \in \mathscr{F}_{+}^{\mathrm{b}} \mid\|v\|_{\mathscr{F}_{+}^{\mathrm{b}}} \leqslant r\right\}
$$

в пространстве $\mathscr{F}_{+}^{\mathrm{b}}$ является компактным множеством в топологии $\Theta_{+}^{\mathrm{w}, \text { loc }}$. Это вытекает из свойств слабой и $*$-слабой компактности ограниченных множеств в соответствующих банаховых пространствах, фигурирующих в определении топологии $\Theta_{+}^{\mathrm{w}, \text { loc }}$ (см., например, [12], [27]). Тогда из общих теорем о компактных топологических пространствах следует, что множество $\mathscr{B}_{r}$, как топологическое подпространство с топологией $\Theta_{+}^{\mathrm{w}, l o c}$, является метризуемым и полным (см., например, [48]). Это свойство будет использоваться при построении траекторного аттрактора РД-системы, а также других эволюционных уравнений с частными производными. (Отметим, что всё пространство $\mathscr{F}_{+}^{\text {loc }}$ с топологией $\Theta_{+}^{\mathrm{w}, \text { loc }}$ не является метризуемым.)

ЗАмечАниЕ 2.4. В сильной топологии $\Theta_{+}^{\text {s,loc }}$ шар $\mathscr{B}_{r}$ компактным не является. Это не позволяет использовать его в качестве поглощающего множества при построении траекторного аттрактора РД-системы. Здесь как раз подходит слабая топология $\Theta_{+}^{\mathrm{w}, \mathrm{loc}}$.

Начнем с изучения пространства траекторий $\mathscr{K}^{+}$РД-системы $(2.1)$ из определения 2.1 . 
УтВЕРЖДЕНИЕ 2.2. Пространство траекторий $\mathscr{K}^{+}$системъ (2.1) замкнуто в топологии $\Theta_{+}^{\mathrm{w}, \text { loc }}$.

Доказательство приведено в [12]. Оно существенно сложнее доказательства аналогичного утверждения 1.1 для системы ОДУ, так как предельный переход в нелинейном члене системы (2.1) необходимо выполнять при условии слабой сходимости последовательности слабых решений.

Ниже мы будем переменную времени часто обозначать $t=s$, и тогда, например, $u(s), s \geqslant 0$, обозначает решение системы (2.1) на полуоси времени.

Рассмотрим полугруппу трансляций $\{T(h)\}:=\{T(h), h \geqslant 0\}$, которая действует в пространстве $\mathscr{F}_{+}^{\text {loc }}$ по формуле:

$$
T(h) v(s)=v(h+s), \quad s \geqslant 0 .
$$

Операторы $\{T(h)\}$ отображают каждое из пространств $\mathscr{F}_{+}^{\text {loc }}$ и $\mathscr{F}_{+}^{\mathrm{b}}$ на себя. То же верно и для шара $\mathscr{B}_{r}$ в $\mathscr{F}_{+}^{\mathrm{b}}$ в силу очевидного неравенства

$$
\|T(h) v\|_{\mathscr{F}_{+}^{\mathrm{b}}} \leqslant\|v\|_{\mathscr{F}_{+}^{\mathrm{b}}}, \quad \forall h \geqslant 0, \quad \forall v \in \mathscr{F}_{+}^{\mathrm{b}} .
$$

Заметим также, что полугруппа $\{T(h)\}$ отображает пространство траекто-

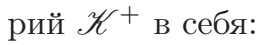

$$
T(h): \mathscr{K}^{+} \rightarrow \mathscr{K}^{+}, \quad \forall h \geqslant 0 .
$$

Действительно, если функция $u(s), s \geqslant 0$, принадлежит пространству $\mathscr{K}^{+}$, то функция $u_{h}(s):=T(h) u(s)=u(h+s), s \geqslant 0$, очевидно, является слабым решением в силу автономности системы $(2.1)$.

Ниже переменная $h$ обозначается через $t(h=t)$, а трансляционная полугруппа обозначается через $\{T(t)\}$.

Имеет место следующее утверждение, которое доказывается полностью аналогично утверждению 1.2 .

УтВеРЖДЕНИЕ 2.3. Полугруппа $\{T(t)\}$ непрерывна в топологии $\Theta_{+}^{\mathrm{w}, \text { loc }}$.

Из неравенств (2.8), (2.9), а также из (2.4) выводится следующее утверждение.

УтВЕРЖДЕНИЕ 2.4. Пространство $\mathscr{K}^{+}$принадлежит $\mathscr{F}_{+}^{\mathrm{b}}$, причем для любой функиии $и(\cdot) \in \mathscr{K}^{+}$справедливо неравенство

$$
\|T(t) u(\cdot)\|_{\mathscr{F}_{+}^{\mathrm{b}}}^{2} \leqslant C|u(0)|^{2} \mathrm{e}^{-\sigma t}+R_{0}^{2}, \quad \forall t \geqslant 0,
$$

где $\sigma=\beta \lambda_{1}$, константа $C$ зависит от матриць а и чисел $\gamma$ и $C_{2}$, а величина $R_{0}$ зависит от $\lambda, C_{1} u\|g\|_{V^{\prime}}$.

Из неравенства (2.15) вытекает, что множество

$$
\mathscr{P}_{0}=\left\{u \in \mathscr{K}^{+} \mid\|u\|_{\mathscr{F}_{+}^{\mathrm{b}}} \leqslant 2 R_{0}\right\}
$$

является поглощающим для полугруппы $\{T(t)\}$ на $\mathscr{K}^{+}$, т. е. для любого множества $\mathfrak{B} \subset \mathscr{K}^{+}$, ограниченного в $\mathscr{F}_{+}^{\mathrm{b}}$, найдется момент времени $t_{1}=t_{1}(\mathfrak{B}) \geqslant 0$ 
такой, что $T(t) \mathfrak{B} \subseteq \mathscr{P}_{0}$ при всех $t \geqslant t_{1}$. Кроме того, в силу неравенств $(2.13)$ и (2.14), это множество инвариантно относительно $\{T(t)\}$ :

$$
T(t) \mathscr{P}_{0} \subseteq \mathscr{P}_{0}, \quad \forall t \geqslant 0 .
$$

Займемся построением траекторного аттрактора трансляционной полугруппы $\{T(t)\}$, действующей на пространстве траекторий $\mathscr{K}^{+}$. Напомним определение притягивающего множества.

Множество $\mathscr{P} \subseteq \mathscr{K}^{+}$называется притягивающим (в топологии $\Theta_{+}^{\mathrm{w}, l o c}$ ) для полугруппы $\{T(t)\}$, если любая окрестность $\mathscr{O}(\mathscr{P})$ множества $\mathscr{P}$ в топологии $\Theta_{+}^{\mathrm{w}, l o c}$ является поглощающим множеством, т. е. для любого ограниченного множества $\mathfrak{B} \subset \mathscr{K}^{+}$найдется $\tau=\tau(\mathfrak{B}, \mathscr{O}) \geqslant 0$ такое, что $T(t) \mathfrak{B} \subseteq \mathscr{O}(\mathscr{P})$ при Bсех $t \geqslant \tau$.

Сформулируем определение траекторного аттрактора полугруппы $\{T(t)\}$, действующей на пространстве траекторий $\mathscr{K}^{+}$(в слабой топологии $\Theta_{+}^{\mathrm{w}, \mathrm{loc}}$ ), которое аналогично определению 1.3 для системы ОДУ.

ОПредЕЛЕНИЕ 2.2. Множество $\mathfrak{A} \subseteq \mathscr{K}^{+}$называется траекторным атmрактором полугруппы $\{T(t)\}$ на $\mathscr{K}^{+}$, если оно ограничено в $\mathscr{F}_{+}^{\mathrm{b}}$, компактно в топологии $\Theta_{+}^{\mathrm{w}, l o c}$, строго инвариантно относительно $\{T(t)\}$, т. е. $T(t) \mathfrak{A}=\mathfrak{A}$ при всех $t \geqslant 0$, и является притягивающим множеством полугруппы $\{T(t)\}$ на $\mathscr{K}^{+}$в топологии $\Theta_{+}^{\mathrm{w}, \text { loc }}$.

Траекторный аттрактор $\mathfrak{A}$ полугруппы $\{T(t)\}$, который также называется траекторным аттрактором РД-системы (2.1), определяется однозначно.

Сформулируем основную теорему о существовании траекторного аттрактора системы (2.1) (см. [11], [12]).

Tеорема 2.1. Пусть $f(v)$ удовлетворяет (2.2), (2.3) и пусть $g(\cdot) \in V^{\prime}$. Тогда трансляиионная полугруппа $\{T(t)\}$, действующая на $\mathscr{K}^{+}$, имеет траекторный аттрактор $\mathfrak{A}$ в топологии $\Theta_{+}^{\mathrm{w}, \text { loc }}$.

ДокАЗАтельство. Рассмотрим на множестве $\mathscr{P}_{0}$ топологию, индуцированную из $\Theta_{+}^{\mathrm{w}, \text { loc }}$. Из утверждения 2.2 и замечания 2.3 вытекает, что $\mathscr{P}_{0}$ замкнуто и компактно в этой топологии. Кроме того, это топологическое пространство метризуемо. Следовательно, полугруппа $\{T(t)\}$ действует на компактном метрическом пространстве $\mathscr{P}_{0}$. При этом полугруппа $\{T(t)\}$ непрерывна в топологии $\Theta_{+}^{\mathrm{w}, \text { loc }}$ на $\mathscr{F}_{+}^{\text {loc }}$ (утверждение 2.3 ), и, тем самым, $\{T(t)\}$ непрерывна на $\mathscr{P}_{0}$. Тогда применима общая теорема о существовании глобального аттрактора $\mathfrak{A} \subseteq \mathscr{P}_{0}$ (см. теорему 1.1 в главе I), который, в силу (I.1.6) задается формулой

$$
\mathfrak{A}=\bigcap_{t \geqslant 0} T(t) \mathscr{P}_{0}
$$

Глобальный аттрактор $\mathfrak{A}$ обладает следующими свойствами: множество $\mathfrak{A}$ ограничено в $\mathscr{F}_{+}^{\mathrm{b}}$, компактно в $\Theta_{+}^{\mathrm{w}, \text { loc }}$, строго инвариантно относительно $\{T(t)\}$, т. е. $T(t) \mathfrak{A}=\mathfrak{A}$ при всех $t \geqslant 0$, и притягивает всё $\mathscr{P}_{0}$, т. е. для любой окрестности $\mathscr{O}(\mathfrak{A})$ найдется $t_{1}=t_{1}(\mathscr{O}) \geqslant 0$ такое, что $T(t) \mathscr{P}_{0} \subseteq \mathscr{O}(\mathfrak{A})$ при всех $t \geqslant t_{1}$. Напомним, что множество $\mathscr{P}_{0}$ является поглощающим для полугруппы $\{T(t)\}$ 
на $\mathscr{K}^{+}$. Поэтому $\mathfrak{A}$ притягивает любое ограниченное (по норме $\mathscr{F}_{+}^{\mathrm{b}}$ ) множество $\mathfrak{B} \subseteq \mathscr{K}^{+}$. Следовательно, множество $\mathfrak{A}$ является траекторным аттрактором. Теорема доказана.

Обозначим через $\Pi_{0, M}$ оператор ограничения на интервал $[0, M]$ функций, заданных на полуоси $\mathbb{R}_{+}$. Отметим, что при любом $M>0$ введенная выше топология $\Theta_{0, M}^{\mathrm{w}}:=\Pi_{0, M} \Theta_{+}^{\mathrm{w}, \text { loc }}$ сильнее, чем топология сходимости по норме в пространстве $L_{2}(0, M ; H):=\left[L_{2}(\Omega \times(0, M))\right]^{N}$. Это вытекает из компактности вложения

$$
L_{2}(0, M ; V) \cap\left\{v \mid \partial_{t} v \in L_{\mathbf{q}}\left(0, M ; \mathbf{H}^{-\mathbf{r}}(\Omega)\right)\right\} \Subset L_{2}(0, M ; H),
$$

которое следует из теоремы Обена (см. [20], [49], [50]).

СлеДСТВИЕ 2.1. Для любого множества $\mathfrak{B} \subset \mathscr{K}^{+}$, ограниченного в $\mathscr{F}_{+}^{\mathrm{b}}$,

$$
\operatorname{dist}_{L_{2}(0, M ; H)}\left(\Pi_{0, M} T(t) \mathfrak{B} ; \Pi_{0, M} \mathfrak{A}\right) \rightarrow 0 \quad(t \rightarrow+\infty), \quad \forall M>0 .
$$

Ядро РД-системы. Траекторный аттрактор эволюционного уравнения удобно описывать с помощью ядра этого уравнения в соответствующем функциональном пространстве.

Рассмотрим РД-систему (2.1) на всей оси времени $s \in \mathbb{R}(-\infty<s<+\infty)$. Мы будем также рассматривать слабые решения $u(x, s)$ этой системы при всех $s \in \mathbb{R}$. Функция

$$
u(\cdot) \in L_{\infty}^{\mathrm{loc}}(\mathbb{R} ; H) \cap L_{2}^{\mathrm{loc}}(\mathbb{R} ; V) \cap L_{\mathbf{p}}^{\mathrm{loc}}\left(\mathbb{R} ; L_{\mathbf{p}}(\Omega)\right)
$$

называется полным слабым решением уравнения (2.1), если она удовлетворяет этому уравнению в смысле пространства $\mathscr{D}^{\prime}\left(\mathbb{R} ; H^{-\mathbf{r}}(\Omega)\right)$. Тогда обобщенная производная по времени $\partial_{t} u(\cdot)$ принадлежит

$$
\mathscr{L}^{\text {loc }}(\mathbb{R}):=\mathbf{L}_{2}^{\text {loc }}\left(\mathbb{R} ; \mathbf{V}^{\prime}\right)+\mathbf{L}_{\mathbf{q}}^{\text {loc }}\left(\mathbb{R} ; \mathbf{L}_{\mathbf{q}}(\Omega)\right) .
$$

Выше были введены пространства $\mathscr{F}_{+}^{\text {loc }}, \mathscr{F}_{+}^{\mathrm{b}} \subset \mathscr{F}_{+}^{\mathrm{loc}}$ и топология $\Theta_{+}^{\mathrm{w}, \text { loc }}$ в $\mathscr{F}_{+}^{\text {loc }}$. Аналогично определяются пространства $\mathscr{F}^{\mathrm{loc}}, \mathscr{F}^{\mathrm{b}} \subset \mathscr{F}^{\text {loc }}$ и топология $\Theta^{\text {w,loc }}$ в $\mathscr{F}^{\text {loc }}$. Следует лишь в этих определениях заменить $\mathbb{R}_{+}$на $\mathbb{R}$. $\mathrm{K}$ примеру, норма в $\mathscr{F}$ b задается следующей формулой:

$$
\|v\|_{\mathscr{F} \mathrm{b}}:=\|v\|_{L_{\infty}(\mathbb{R} ; H)}+\|v\|_{L_{2}^{\mathrm{b}}(\mathbb{R} ; V)}+\|v\|_{L_{\mathbf{p}}^{\mathrm{b}}\left(\mathbb{R} ; L_{\mathbf{p}}(\Omega)\right)}+\left\|\partial_{t} v\right\|_{\mathscr{L}^{\mathrm{b}}(\mathbb{R})} .
$$

ОПРеДЕЛЕНИЕ 2.3. Ядром $\mathscr{K}$ РД-системы (2.1) называется совокупность всех ее полных слабых решений $u(s), s \in \mathbb{R}$, имеющих ограниченную норму B $\mathscr{F}^{\mathrm{b}}$.

Из определения ядра $\mathscr{K}$ вытекает, что если $u \in \mathscr{K}$, то $T(h) u \in \mathscr{K}$ при любом $h \in \mathbb{R}$ (напомним, что $T(h) u(s)=u(h+s)$ ), и все ядро строго инвариантно относительно трансляционной группы $\{T(h), h \in \mathbb{R}\}$ :

$$
T(h) \mathscr{K}=\mathscr{K}, \quad \forall h \in \mathbb{R} .
$$

Обозначим через $\Pi_{+}$оператор ограничения на полуось $\mathbb{R}_{+}$функций $f(s)$, $s \in \mathbb{R}$, заданных на всей оси $\mathbb{R}: \Pi_{+} f(s)=f(s)$ при $s \geqslant 0$. 
ТеОрема 2.2. Ядро $\mathscr{K}$ и траекторный аттрактор А системы (2.1) связаны следующим соотношением:

$$
\mathfrak{A}=\Pi_{+} \mathscr{K} .
$$

Ядро $\mathscr{K}$ ограничено в пространстве $\mathscr{F}^{\mathrm{b}}$ и компактно в топологии $\Theta^{\mathrm{loc}}$.

ДокАЗАтЕЛьство. Легко видеть, что для любой функции $u \in \mathscr{K}$ функция $\Pi_{+} u \in \mathscr{K}^{+}$, и, тем самым, $\Pi_{+} \mathscr{K} \subset \mathscr{K}^{+}$. Рассмотрим траекторный аттрактор $\mathfrak{A} \subset \mathscr{K}^{+}$уравнения (2.1).

Для любой функции $u \in \mathscr{K}$ множество траекторий $\mathfrak{B}_{u}=\left\{\Pi_{+} u(s+h), s \geqslant 0 \mid\right.$ $h \in \mathbb{R}\} \subset \mathscr{K}^{+}$является строго инвариантным относительно полугруппы $\{T(h), h \geqslant 0\}$ :

$$
T(h) \mathfrak{B}_{u}=\mathfrak{B}_{u}, \quad \forall h \geqslant 0 .
$$

Кроме того, множество $\mathfrak{B}_{u}$ ограничено в $\mathscr{F}_{+}^{\mathrm{b}}$, так как при любом $h \in \mathbb{R}$

$$
\left\|\Pi_{+} T(h) u\right\|_{\mathscr{F}_{+}^{\mathrm{b}}} \leqslant\|T(h) u\|_{\mathscr{F} \mathrm{b}} \equiv\|u\|_{\mathscr{F} \mathrm{b}} \quad \Longrightarrow \quad\left\|\mathfrak{B}_{u}\right\|_{\mathscr{F}_{+}^{\mathrm{b}}} \leqslant\|u\|_{\mathscr{F} \mathrm{b}} .
$$

Тогда траекторный аттрактор $\mathfrak{A}$ притягивает $T(t) \mathfrak{B}_{u}$ при $t \rightarrow+\infty$ в топологии $\Theta_{+}^{\mathrm{w}, \text { loc }}$, и из $(2.21)$ находим, что $\mathfrak{B}_{u} \subseteq \mathfrak{A}$ при любом $u \in \mathscr{K}$ (так как $\mathfrak{A}-$ компактное множество) и, в частности, $\Pi_{+} u \in \mathfrak{A}$. Мы доказали, что

$$
\Pi_{+} \mathscr{K} \subseteq \mathfrak{A}
$$

Убедимся в том, что обратное включение также имеет место. Действительно, пусть $u_{0} \in \mathfrak{A}$. Функция $u_{0}(s)$ задана при $s \geqslant 0$. В силу строгой инвариантности аттрактора $\mathfrak{A}$ найдется функция $u_{-1} \in \mathfrak{A}$ такая, что $T(1) u_{-1}=u$. Определим $u(s):=u_{-1}(s+1)$ при $s \geqslant-1$. Очевидно, что $u(s) \equiv u_{0}(s)$ при $s \geqslant 0$, и при этом $u(s)$ является слабым решением (2.1) уже при $s \geqslant-1$. Продолжая этот процесс до бесконечности, находим функцию $u(s), s \in \mathbb{R}$, которая является полным слабым решением (2.1), причем $u(s) \equiv u_{0}(s)$ при $s \geqslant 0$. Кроме того, из построения функции $u(s)$ видно, что

$$
\|u\|_{\mathscr{F} \mathrm{b}} \leqslant\|\mathfrak{A}\|_{\mathscr{F}+} .
$$

Значит, функция $u$ принадлежит ядру $\mathscr{K}$, причем $u_{0}=\Pi_{+} u$, и, следовательно,

$$
\mathfrak{A} \subseteq \Pi_{+} \mathscr{K} .
$$

Равенство (2.20) доказано.

Сильный траекторный аттрактор РД-системы. Изучим построенный траекторный аттрактор в сильной топологии $\Theta_{+}^{\text {s,loc }}$. Определение сильного траекторного аттрактора получается, если в определении 2.2 условие слабого притяжения заменить на условие сильного притяжения в топологии $\Theta_{+}^{\mathrm{s}, \mathrm{loc}}$.

Отметим, что из утверждения 2.2 следует, что пространство траекторий $\mathscr{K}^{+}$ замкнуто и в сильной топологии $\Theta_{+}^{\mathrm{s}, \text { loc }}$. Полугруппа $\{T(t)\}$, очевидно, непрерывна также и в топологии $\Theta_{+}^{\mathrm{s}, \text { loc }}$. 
Теорема 2.3. Траекторный аттрактор $\mathfrak{A}$ в слабой топологии $\Theta_{+}^{\mathrm{w}, \text { loc }}$, построенный в теореме 2.1, одновременно является силъным траекторным аттрактором полугруппы $\{T(t)\}$, действующей на пространстве траекторий $\mathscr{K}^{+}$изучаемой РД-системы (2.1).

Доказательство этой теоремы приведено в [51]. Оно основано на методе энергетических тождеств (см. [52], [53]), который часто используется при построении глобальных аттракторов некомпактных полугрупп для диссипативных эволюционных уравнений в неограниченных областях, обладающих свойством единственности решения задачи Коши.

Глобальный аттрактор РД-системы. Для системы (2.1) можно построить глобальный аттрактор в пространстве $H$, имея траекторный аттрактор, подобно тому как это было сделано в разделе 1 для системы ОДУ, не используя свойство единственности решений задачи Коши.

Ранее отмечалось, что любое слабое решения $u(t), t \geqslant 0$, системы $(2.1)$ удовлетворяет тождеству $(2.7)$, причем $u \in C\left(\mathbb{R}_{+} ; H\right)$. Рассмотрим теперь любое ограниченное в $\mathscr{F}_{+}^{\mathrm{b}}$ множество траекторий $\mathfrak{B} \subset \mathscr{K}^{+}$. Тогда оно ограничено также в пространстве $C_{\mathrm{b}}\left(\mathbb{R}_{+} ; H\right)$. Обозначим через $\mathfrak{B}(t)$ подмножество в $H$, которое состоит из значений всех функций из множества $\mathfrak{B}$ в момент времени $t \geqslant 0$ :

$$
\mathfrak{B}(t)=\{u(t) \mid u \in \mathfrak{B}\} \subset H .
$$

Очевидно, что множество $\mathfrak{B}(t)$, которое называется сечением $\mathfrak{B}$ в момент $t$, равномерно (по $t \geqslant 0)$ ограничено в $H$.

Пусть $\mathfrak{A}$ - траекторный аттрактор системы реакции-диффузии. Рассмотрим его сечение в момент времени $t=0$ :

$$
\mathfrak{A}(0):=\{u(0) \mid u \in \mathfrak{A}\} \subset H .
$$

Из строгой инвариантности множества $\mathfrak{A}$ относительно полугруппы трансляций $\{T(t)\}$ следует, что

$$
\mathfrak{A}(0)=\mathfrak{A}(t), \quad \forall t \geqslant 0,
$$

причем $\mathfrak{A}(0)$ ограничено в $H$.

ТЕОРема 2.4. Множество $\mathfrak{A}(0)$ компактно в $H$, и для любого множества траекторий $\mathfrak{B} \subset \mathscr{K}^{+}$, ограниченного в $\mathscr{F}_{+}^{\mathrm{b}}$, его сечения $\mathfrak{B}(t)$ притягиваятся $\kappa \mathfrak{A}(0)$ при $t \rightarrow+\infty$ в сильной топологии $H$, m.e.

$$
\operatorname{dist}_{H}(\mathfrak{B}(t), \mathfrak{A}(0)) \rightarrow 0 \quad(t \rightarrow+\infty) .
$$

Для доказательства достаточно воспользоваться теоремой 2.3, в которой, в частности, доказано притяжение множеств $T(t) \mathfrak{B}$ к аттрактору $\mathfrak{A}$ в сильной топологии $C^{\text {loc }}\left(\mathbb{R}_{+} ; H\right)$ при $t \rightarrow+\infty$, и, следовательно, при любом $M \geqslant 0$

$$
\operatorname{dist}_{C([0, M] ; H)}\left(\Pi_{0, M} T(t) \mathfrak{B} ; \Pi_{0, M} \mathfrak{A}\right) \rightarrow 0 \quad(t \rightarrow+\infty) .
$$

Тогда при $M=0$ мы получаем соотношение (2.23) (аналогичный результат получен в [54]). 
Теорема 2.4 позволяет заключить, что множество

$$
\mathscr{A}:=\mathfrak{A}(0)
$$

является глобальным аттрактором системы (2.1), который определяется аналогично глобальному аттрактору системы ОДУ (см. определение 1.4).

ОПРЕДЕЛЕНИЕ 2.4. Множество $\mathscr{A} \subset H$ называется глобалъным аттрактором системы (2.1), если оно компактно в $H$ и при этом

1) для любого множества $\mathfrak{B} \subset \mathscr{K}^{+}$, ограниченного по норме $\mathscr{F}_{+}^{\mathrm{b}}$, его сечение $\mathfrak{B}(t)$ притягивается при $t \rightarrow+\infty$ к множеству $\mathscr{A}$ по норме $H$, т. е.

$$
\operatorname{dist}_{H}(\mathfrak{B}(t), \mathscr{A}) \rightarrow 0 \quad(t \rightarrow+\infty)
$$

2) множество $\mathscr{A}$ является минимальным компактным множеством в пространстве $H$, которое притягивает при $t \rightarrow+\infty$ сечения всех ограниченных множеств из $\mathscr{K}^{+}$по норме $H$.

Отметим, что глобальный аттрактор определен однозначно.

ЗАмЕЧАНИЕ 2.5. Если для системы реакции-диффузии имеет место теорема единственности решений задачи Коши и для нее можно построить обычную полугруппу $\{S(t)\}$ в пространстве начальных условий $H$, то множество $\mathscr{A}$ из теоремы 2.3 является обычным глобальным аттрактором этой полугруппы (см. определение 1.2 в гл. I) и в соотношении $(2.24) \mathfrak{B}(t)=S(t) \mathfrak{B}(0)=S(t) B$, где $B$ - произвольное ограниченное в $H$ множество начальных условий.

\section{3. Двумерная система Эйлера с диссипацией и ее траекторный аттрактор}

Слабые решения системы Эйлера с диссипацией. Рассматривается следующая 2D-система уравнений Эйлера с диссипацией:

$$
\begin{gathered}
\partial_{t} u+B(u, u)+r u=g(x), \quad(\nabla, u):=\partial_{x_{1}} u^{1}+\partial_{x_{2}} u^{2}=0, \\
u=\left(u^{1}(x, t), u^{2}(x, t)\right), \quad x=\left(x_{1}, x_{2}\right) \in \mathbb{T}^{2}, \quad t \geqslant 0,
\end{gathered}
$$

где $\mathbb{T}^{2}=[\mathbb{R} \bmod 2 \pi]^{2}-$ двумерный тор, $B(u, u)=P\left(u^{1} \partial_{x_{1}} u+u^{2} \partial_{x_{2}} u\right)$ и $P$ является ортогональным оператором Лерэ, который проецирует пространство $\left[L_{2}\left(\mathbb{T}^{2}\right)\right]^{2}$ на подпространство

$$
H=\left[\left\{v(x) \in\left[C^{\infty}\left(\mathbb{T}^{2}\right)\right]^{2} \mid(\nabla, v)=0\right\}\right]_{\left[L_{2}\left(\mathbb{T}^{2}\right)\right]^{2}}
$$

Аналогично вводится пространство

$$
H^{1}=\left[\left\{v(x) \in\left[C^{\infty}\left(\mathbb{T}^{2}\right)\right]^{2} \mid(\nabla, v)=0\right\}\right]_{\left[H^{1}\left(\mathbb{T}^{2}\right)^{2}\right]},
$$

которое вкладывается в стандартную шкалу пространств $H^{s}, s \in \mathbb{R}$, где $H^{0}=H$, и $H^{-s}=\left(H^{s}\right)^{*}$ - сопряженное пространство к $H^{s}, s \geqslant 0$. Нормы в пространствах $H$ и $H^{1}$ обозначаются $|\cdot|$ и $\|\cdot\|$ соответственно. Отметим, что $H^{1} \Subset H$. 
В уравнении (3.1) число $r>0$ является коэффициентом диссипации. Давление $p(x, t)$ исключено из системы с помощью применения к обеим ее частям оператора $P$. Предполагается, что внешняя сила $g$ принадлежит $H^{1}$.

Двумерная система Эйлера с диссипацией (3.1) рассматривалась в ряде статей (см., например, [55]-[57]). Уравнения (3.1) описывают геофизические процессы в атмосфере и океане (см., например, [58; гл. 4]).

Начальное условие задается при $t=0$ :

$$
\left.u\right|_{t=0}=u_{0}, \quad u_{0} \in H^{1} .
$$

Существование решения $\left\{u(t), t \in \mathbb{R}_{+}\right\}$задачи (3.1), (3.2) доказывается с помощью метода Галёркина. При этом целесообразно в качестве базиса взять ортогональную (в $H)$ систему собственных функций $\left\{e_{j}(x)=\left(e_{j}^{1}(x), e_{j}^{2}(x)\right) \in\right.$ $\left.H^{2}, j=0,1,2, \ldots\right\}$ оператора Стокса:

$$
-P \Delta e_{j}(x)=\lambda_{j} e_{j}(x), \quad\left(\nabla, e_{j}(x)\right)=0, \quad x \in \mathbb{T}^{2}, \quad j=0,1,2, \ldots
$$

Отметим, что при периодических граничных условиях имеем $P \Delta \equiv \Delta$ (см., например, [2]). Напомним, что $e_{0}(x) \equiv e_{0}$ является постоянным вектором, а спектр оператора Стокса имеет вид $0=\lambda_{0}<\lambda_{1}<\lambda_{2} \leqslant \cdots, \lambda_{j} \rightarrow+\infty$ при $j \rightarrow \infty$.

Приближенное решение в методе Галёркина ищется в виде

$$
u_{m}(t)=\sum_{j=0}^{m} c_{j, m}(t) e_{j}, \quad m=1,2, \ldots,
$$

где $c_{j, m}(t)$ - неизвестные скалярные функции, причем функция $u_{m}(x, t)$ удовлетворяет уравнению

$$
\partial_{t} u_{m}+\Pi_{m} B\left(u_{m}, u_{m}\right)+r u_{m}=\Pi_{m} g,
$$

которое эквивалентно соответствующей системе ОДУ относительно функций $c_{j, m}(t), j=0,1, \ldots, m$. В (3.4) $\Pi_{m}$ обозначает оператор ортогонального проецирования из $H$ на конечномерное подпространство $\left[e_{0}(x), \ldots, e_{m}(x)\right]$. При $t=0$ задается начальное условие

$$
\left.u_{m}\right|_{t=0}=u_{m}(0)=\Pi_{m} u_{0},
$$

где $u_{0}$ - такое же, как в $(3.2)$.

Задача Коши (3.4)-(3.5) имеет и притом единственное решение $u_{m}(t) \in$ $C^{1}\left(\left[0, \tau_{m}\right) ; H^{2}\right)$ для некоторого $\tau_{m}>0$. Умножим уравнение $(3.4)$ на $u_{m}(t)$ скалярно в $H$ и воспользуемся известным тождеством (см. [2], [27])

$$
(B(u, u), u)=0, \quad u \in H^{1} .
$$

После элементарных преобразований получим

$$
\frac{1}{2} \frac{d}{d t}\left|u_{m}(t)\right|^{2}+r\left|u_{m}(t)\right|^{2}=\left(g, u_{m}(t)\right), \quad \forall t \in\left[0, \tau_{m}\right)
$$


где $|u|=\|u\|_{\left[L_{2}\left(\mathbb{T}^{2}\right)\right]^{2}}$. Из дифференциального соотношения (3.7) следует неравенство

$$
\left|u_{m}(t)\right|^{2} \leqslant\left|u_{m}(0)\right|^{2} \mathrm{e}^{-r t}+r^{-1}|g|^{2} \leqslant\left|u_{0}\right|^{2} \mathrm{e}^{-r t}+r^{-1}|g|^{2}, \quad \forall t \in\left[0, \tau_{m}\right) .
$$

Умножив скалярно в $H$ обе части уравнения (3.4) на $-P \Delta u_{m}=-\Delta u_{m}$ и воспользовавшись тождествами $-\left(u_{m}, \Delta u_{m}\right)=\left|\nabla u_{m}\right|^{2}$ и $-\left(g, \Delta u_{m}\right)=\left(\nabla g, \nabla u_{m}\right)$, получаем равенство

$\frac{1}{2} \frac{d}{d t}\left|\nabla u_{m}(t)\right|^{2}-\left(B\left(u_{m}, u_{m}\right), \Delta u_{m}\right)+r\left|\nabla u_{m}(t)\right|^{2}=\left(\nabla g, \nabla u_{m}(t)\right), \quad \forall t \in\left[0, \tau_{m}\right)$.

Как известно, в случае периодических граничных условий $\left(x \in \mathbb{T}^{2}\right)$ справедливо тождество

$$
(B(u, u), \Delta u)=0, \quad \forall u \in H^{2}
$$

(см. [2; гл. VI, лемма 3.1] и [57]), которое играет ключевую роль в дальнейших построениях. Из (3.9) и (3.10) вытекает равенство

$$
\frac{1}{2} \frac{d}{d t}\left|\nabla u_{m}(t)\right|^{2}+r\left|\nabla u_{m}(t)\right|^{2}=\left(\nabla g, \nabla u_{m}(t)\right), \quad \forall t \in\left[0, \tau_{m}\right) .
$$

Из (3.11) получается неравенство

$$
\left|\nabla u_{m}(t)\right|^{2} \leqslant\left|\nabla u_{m}(0)\right|^{2} \mathrm{e}^{-r t}+r^{-1}|\nabla g|^{2}, \quad \forall t \in\left[0, \tau_{m}\right) .
$$

Сложив неравенства (3.8) и (3.12), приходим к основной оценке

$$
\left\|u_{m}(t)\right\|^{2} \leqslant\left\|u_{m}(0)\right\|^{2} \mathrm{e}^{-r t}+r^{-1}\|g\|^{2} \leqslant\left\|u_{0}\right\|^{2} \mathrm{e}^{-r t}+r^{-1}\|g\|^{2}
$$

при всех $t \in\left[0, \tau_{m}\right)$, где, напомним, $\|v\|^{2}=\|v\|_{H^{1}}^{2}=|v|^{2}+|\nabla v|^{2}$. Из неравенства (3.13) следует, что решение $u_{m}(x, t)$ задачи $(3.4)-(3.5)$ продолжается на всю полуось $\mathbb{R}_{+}$(т. е. $\left.\tau_{m}=+\infty\right), u_{m}(x, t) \in C_{\mathrm{b}}^{1}\left(\mathbb{R}_{+} ; H^{1}\right)$ и

$$
\left\|u_{m}(t)\right\|^{2} \leqslant\left\|u_{0}\right\|^{2}+r^{-1}\|g\|^{2}, \quad \forall t \geqslant 0,
$$

при любом $m \in \mathbb{N}$. В частности,

$$
\left\|u_{m}\right\|_{L_{\infty}\left(\mathbb{R}_{+} ; H^{1}\right)}^{2}:=\operatorname{ess} \sup \left\{\left\|u_{m}(t)\right\|^{2} \mid t \geqslant 0\right\} \leqslant\left\|u_{0}\right\|^{2}+r^{-1}\|g\|^{2}, \quad \forall m \in \mathbb{N} .
$$

Поскольку $u_{0} \in H^{1}$, то начальное условие задачи (3.4)-(3.5)

$$
u_{m}(0)=\Pi_{m} u_{0} \rightarrow u_{0} \quad(m \rightarrow \infty) \quad \text { сильно в } H^{1} .
$$

Неравенство (3.14) влечет существование подпоследовательности $\left\{m^{\prime}\right\} \subset$ $\{m\}$ такой, что

$$
u_{m^{\prime}}(t) \rightarrow u(t) \quad\left(m^{\prime} \rightarrow \infty\right) \quad * \text {-слабо в } L_{\infty}^{\text {loc }}\left(\mathbb{R}_{+} ; H^{1}\right)
$$

для некоторой функции $u(\cdot) \in L_{\infty}\left(\mathbb{R}_{+} ; H^{1}\right)$. 
Утверждается, что $u(t)$ является слабым решением задачи (3.1)-(3.2). В самом деле, из уравнений (3.4) и оценки (3.14) находим, что

$$
\begin{aligned}
\left\|\partial_{t} u_{m}(t)\right\|_{H^{-1}} & \leqslant\left\|B\left(u_{m}(t), u_{m}(t)\right)\right\|_{H^{-1}}+r\left\|u_{m}(t)\right\|_{H^{-1}}+\|g\|_{H^{-1}} \\
& \leqslant C\left(\left\|u_{m}(t)\right\|_{\left[L_{4}\left(\mathbb{T}^{2}\right)\right]^{2}}^{2}+\left\|u_{m}(t)\right\|+\|g\|\right) \leqslant C_{1}\left(\left\|u_{m}(t)\right\|^{2}+\|g\|+1\right) \\
& \leqslant C_{1}\left(\left\|u_{0}\right\|^{2}+r^{-1}\|g\|^{2}+\|g\|+1\right), \quad t \geqslant 0 .
\end{aligned}
$$

Здесь использовались известные неравенства

$$
\left\|B\left(u_{m}, u_{m}\right)\right\|_{H^{-1}} \leqslant c\left\|u_{m}\right\|_{\left[L_{4}\left(\mathbb{T}^{2}\right)\right]^{2}}^{2}, \quad\left\|u_{m}\right\|_{\left[L_{4}\left(\mathbb{T}^{2}\right)\right]^{2}}^{2} \leqslant c^{\prime}\left\|u_{m}\right\|^{2} .
$$

Из (3.16) и (3.17) следует, что

$$
\partial_{t} u_{m^{\prime}}(t) \rightarrow \partial_{t} u(t) \quad\left(m^{\prime} \rightarrow \infty\right) \quad \text { *-слабо в } L_{\infty}^{\text {loc }}\left(\mathbb{R}_{+} ; H^{-1}\right) .
$$

Применяя (3.16), (3.19) и теорему Обена о компактности (см. [20], [49], [50]), получаем сильную сходимость:

$$
u_{m^{\prime}}(t) \rightarrow u(t) \quad\left(m^{\prime} \rightarrow \infty\right) \quad \text { сильно в } L_{\infty}^{\text {loc }}\left(\mathbb{R}_{+} ; H\right),
$$

а из (3.19) и (3.20) заключаем (используя стандартный прием при предельном переходе в нелинейном члене, аналогично [20], [25], [26]), что

$$
B\left(u_{m^{\prime}}, u_{m^{\prime}}\right) \rightarrow B(u, u) \quad\left(m^{\prime} \rightarrow \infty\right) \quad \text { *-слабо } L_{\infty}^{\text {loc }}\left(\mathbb{R}_{+} ; H^{-1}\right) .
$$

Теперь, имея соотношения (3.16), (3.19) и (3.21), можно совершить предельный переход при $m^{\prime} \rightarrow \infty$ в уравнении (3.4) в пространстве обобщенных функций $D^{\prime}\left(\mathbb{R}_{+} ; H^{-1}\right)$ (см. [20]). Получаем, что функция $u(t)$ является обобщенным (слабым) решением уравнения (3.1) в пространстве $D^{\prime}\left(\mathbb{R}_{+} ; H^{-1}\right)$, причем $\partial_{t} u(x, t)$ принадлежит $L_{\infty}\left(\mathbb{R}_{+} ; H^{-1}\right)$, а из (3.15) вытекает, что $u(t)$ удовлетворяет начальному условию (3.2). Наконец, из неравенства (3.13) заключаем, что построенное решение $u(x, t)$ удовлетворяет оценке

$$
\|u(t)\|^{2} \leqslant\|u(0)\|^{2} \mathrm{e}^{-r t}+r^{-1}\|g\|^{2}, \quad \forall t \in \mathbb{R}_{+} .
$$

Доказано следующее утверждение.

УтвеРЖДЕНИЕ 3.1. Для любого $u_{0} \in H^{1}$ задача Коши (3.1)-(3.2) имеет слабое решение $u(t) \in L_{\infty}\left(\mathbb{R}_{+} ; H^{1}\right)$, причем $\partial_{t} u(t) \in L_{\infty}\left(\mathbb{R}_{+} ; H^{-1}\right)$, и выполнено неравенство (3.22).

ЗАмечАниЕ 3.1. Любое слабое решение $u(t) \in L_{\infty}\left(\mathbb{R}_{+} ; H^{1}\right)$ уравнения $(3.1)$ удовлетворяет энергетическому тождеству

$$
\frac{1}{2} \frac{d}{d t}|u(t)|^{2}+r|u(t)|^{2}=(g, u(t)), \quad \forall t \geqslant 0,
$$

где скалярная функция $|u(t)|^{2}$ является абсолютно непрерывной (см. (3.7)). Однако для выполнения аналогичного тождества (см. (3.11)) для функции $|\nabla u(t)|^{2}, t \geqslant 0$ (или неравенства вида (3.12), вытекающего из этого тождества) гладкости любого слабого решения уже не хватает. 
ЗАмЕчАниЕ 3.2. Проблема единственности для задачи (3.1)-(3.2) в классе слабых решений из $L_{\infty}\left(\mathbb{R}_{+} ; H^{1}\right)$ остается нерешенной. Аналогичная картина наблюдается и для классической консервативной 2D-системы Эйлера (3.1) при $r=0$, для которой теоремы существования и единственности решений доказаны в классе функций $u(x, t)$ с вихрем $\nabla \times u:=\partial_{x_{1}} u^{2}-\partial_{x_{2}} u^{1} \in L_{\infty}\left(\mathbb{R}_{+} ; L_{\infty}\left(\mathbb{T}^{2}\right)\right)$ при условии, что $\nabla \times g, \nabla \times u_{0} \in L_{\infty}\left(\mathbb{T}^{2}\right)$ (см. [59]-[61]). Эти результаты легко распространяются на уравнение (3.1) при $r>0$. Тем не менее ниже будет показано, что теорема единственности не нужна для построения траекторного аттрактора двумерной системы Эйлера с диссипацией. При этом никаких дополнительных условий для вихря функции $u(t)$ не предполагается.

Траекторный и глобальный аттракторы системы Эйлера с диссипацией. Вводятся пространства

$$
\begin{gathered}
\mathscr{F}_{+}^{\mathrm{loc}}=\left\{v(s), s \in \mathbb{R}_{+} \mid v \in L_{\infty}^{\mathrm{loc}}\left(\mathbb{R}_{+} ; H^{1}\right), \partial_{t} v \in L_{\infty}^{\mathrm{loc}}\left(\mathbb{R}_{+} ; H^{-1}\right)\right\} \\
\mathscr{F}_{+}^{\infty}=\left\{v(s), s \in \mathbb{R}_{+} \mid v \in L_{\infty}\left(\mathbb{R}_{+} ; H^{1}\right), \partial_{t} v \in L_{\infty}\left(\mathbb{R}_{+} ; H^{-1}\right)\right\} .
\end{gathered}
$$

Напомним, что $z(\cdot) \in L_{\infty}^{\text {loc }}\left(\mathbb{R}_{+} ; E\right)$, если и только если $z(\cdot) \in L_{\infty}(0, M ; E)$ при каждом $M>0$. Очевидно, что $\mathscr{F}_{+}^{\infty} \subset \mathscr{F}_{+}^{\text {loc }}$. Норма в пространстве $\mathscr{F}_{+}^{\infty}$ задается формулой

$$
\|v\|_{\mathscr{F}_{+}^{\infty}}:=\|v\|_{L_{\infty}\left(\mathbb{R}_{+} ; H^{1}\right)}+\left\|\partial_{t} v\right\|_{L_{\infty}\left(\mathbb{R}_{+} ; H^{-1}\right)} .
$$

В пространстве $\mathscr{F}_{+}^{\text {loc }}$ вводится (“слабая") топология $\Theta_{+}^{\mathrm{w}, l o c}$, порожденная следующей сходимостью последовательностей $\left\{v_{n}(s)\right\} \subset \mathscr{F}_{+}$: пс по определению, $v_{n}(\cdot) \rightarrow v(\cdot)(n \rightarrow \infty)$ в топологии $\Theta_{+}^{\mathrm{w}, \text { lос }}$, если для любого $M>0$

(a) $v_{n}(s) \neg v(s)(n \rightarrow \infty) *$-слабо в $L_{\infty}\left(0, M ; H^{1}\right)$ и

(b) $\partial_{t} v_{n}(s) \rightarrow \partial_{t} v(s)(n \rightarrow \infty) *$-слабо в $L_{\infty}\left(0, M ; H^{-1}\right)$.

Топология $\Theta_{+}^{\mathrm{w}, l o c}$ является метризуемой на любом шаре

$$
\mathscr{B}_{R}=\left\{v \in \mathscr{F}_{+}^{\infty} \mid\|v\|_{\mathscr{F}_{+}^{\infty}} \leqslant R\right\}
$$

в пространстве $\mathscr{F}_{+}^{\infty}$ (см. замечание 2.3 , а также [12], [48]). Соответствующую метрику для $\mathscr{B}_{R}$ обозначим через $\rho(\cdot, \cdot)$. Отметим, что во всем пространстве $\mathscr{F}_{+}^{\text {loc }}$ топология $\Theta_{+}^{\mathrm{w}, \text { loc }}$ метризуемой не является.

Определим теперь траекторное пространство $\mathscr{K}^{+}(N)$ для уравнения (3.1), которое зависит от числа $N \geqslant 0$.

ОПредЕлениЕ 3.1. Функция $u(s) \in \mathscr{F}_{+}^{\infty}$ принадлежит $\mathscr{K}^{+}(N)$, если

1) $u(s)$ является обобщенным решением $(3.1)$ в пространстве $D^{\prime}\left(\mathbb{R}_{+} ; H^{-1}\right)$;

2) $u(s)$ удовлетворяет неравенству

$$
\|u(s)\|^{2} \leqslant N \mathrm{e}^{-r s}+r^{-1}\|g\|^{2}, \quad \forall s \in \mathbb{R}_{+} .
$$

Отметим, что пространство $\mathscr{K}^{+}(N)$ непусто при любом $N \geqslant 0$. Действительно, если $u_{0} \in H^{1}$ и $\left\|u_{0}\right\|^{2} \leqslant N$, то решение $u(x, t)$ задачи $(3.1)-(3.2)$, полученное по методу Галёркина (см. утверждение 3.1$)$, является обобщенным решением системы $(3.1)$ в $D^{\prime}\left(\mathbb{R}_{+} ; H^{-1}\right)$, и оно удовлетворяет (3.26) (см. (3.22) при $\left.\left\|u_{0}\right\|^{2} \leqslant N\right)$. Следовательно, $u(x, t)$ принадлежит $\mathscr{K}^{+}(N)$. 
В [62] доказано, что пространство $\mathscr{K}^{+}(N)$ ограничено в $\mathscr{F}_{+}^{\infty}$ и замкнуто в топологии $\Theta_{+}^{\text {w,loc }}$ при любом $N \geqslant 0$. Пространство $\mathscr{K}^{+}(N)$ является компактным метрическим пространством в топологии $\Theta_{+}^{\mathrm{w}, \text { loc }}$, так как $\mathscr{K}^{+}(N)$ содержится в некотором шаре $\mathscr{B}_{R}$ радиуса $R=R(N)$ пространства $\mathscr{F}_{+}^{\infty}$.

Рассмотрим теперь трансляционную полугруппу $\{T(t)\}:=\{T(t), t \geqslant 0\}$,

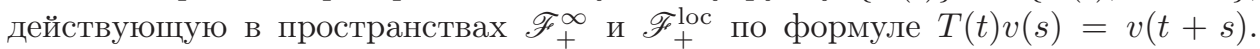
Полугруппа $\{T(t)\}$ непрерывна в топологии $\Theta_{+}^{\mathrm{w}, \text { loc }}$. Эта полугруппа действует также в траекторном пространстве $\mathscr{K}^{+}(N)$ уравнения (3.1). Легко проверить, что

$$
T(t) \mathscr{K}^{+}(N) \subseteq \mathscr{K}^{+}(N), \quad \forall t \geqslant 0 .
$$

Следовательно, непрерывная полугруппа $\{T(t)\}$ действует на компактном метрическом пространстве $\mathscr{K}^{+}(N)$. По теореме 1.1 главы I заключаем, что полугруппа $\left.\{T(t)\}\right|_{\mathscr{K}+(N)}$ имеет глобальный аттрактор $\mathfrak{A}_{N} \subseteq \mathscr{K}^{+}(N)$, который называется траекторным аттрактором уравнения (3.1) (см. также [12], [11]). При этом имеет место формула

$$
\mathfrak{A}_{N}=\bigcap_{t \geqslant 0} T(t) \mathscr{K}^{+}(N) .
$$

Напомним, что множество $\mathfrak{A}_{N}$ строго инвариантно относительно полугруппы $\{T(t)\}: T(t) \mathfrak{A}_{N}=\mathfrak{A}_{N}$ при всех $t \geqslant 0$, и для любого множества траекторий $\mathfrak{B} \subseteq \mathscr{K}^{+}(N)$ выполнено соотношение

$$
\operatorname{dist}_{\rho}\left(T(t) \mathfrak{B}, \mathfrak{A}_{N}\right) \rightarrow 0 \quad(t \rightarrow+\infty) .
$$

В [62] установлено, что при любом $N \geqslant 0$ траекторный аттрактор $\mathfrak{A}(N)$ не зависит от $N, \mathfrak{A}_{N}=\mathfrak{A}_{0}=: \mathfrak{A}$ и

$$
\operatorname{dist}_{\rho}(T(t) \mathfrak{B}, \mathfrak{A}) \rightarrow 0 \quad(t \rightarrow+\infty), \quad \forall \mathfrak{B} \subseteq \mathscr{K}^{+}(N), \quad \forall N \geqslant 0 .
$$

Kроме того, $\mathfrak{A} \subseteq \mathscr{K}^{+}(0)$, т. е.

$$
\|u(\cdot)\|_{L_{\infty}\left(\mathbb{R}_{+} ; H^{1}\right)}^{2} \leqslant r^{-1}\|g\|^{2}, \quad \forall u \in \mathfrak{A},
$$

а из неравенства (3.17) получаем, что

$$
\left\|\partial_{t} u(\cdot)\right\|_{L_{\infty}\left(\mathbb{R}_{+} ; H^{-1}\right)} \leqslant C_{1}\left(\left(1+r^{-1}\right)\|g\|^{2}+1\right), \quad \forall u \in \mathfrak{A} .
$$

Пространства $\mathscr{F}^{\text {loc }}$ и $\mathscr{F}^{\infty}$ определяются аналогично $\mathscr{F}_{+}^{\text {loc }}$ и $\mathscr{F}_{+}^{\infty}$ (для этого следует заменить в (3.23) и (3.24) полупрямую $\mathbb{R}_{+}$на всю прямую $\left.\mathbb{R}\right)$. В пространстве $\mathscr{F}^{\text {loc }}$ задается топология $\Theta^{\mathrm{w}, \mathrm{loc}}$ аналогично топологии $\Theta_{+}^{\mathrm{w}, \text { loc }}$ на $\mathscr{F}_{+}^{\text {loc }}$. Через $\Pi_{+}$обозначается оператор ограничения на полуось $\mathbb{R}_{+}$.

Ядро $\mathscr{K}$ системы (3.1) состоит из всех функций $\{u(s), s \in \mathbb{R}\} \in \mathscr{F}$, которые являются ограниченными (в $H^{1}$ ) полными обобщенными решениями (3.1) на всей оси времени $\mathbb{R}$, удовлетворяющими неравенству

$$
\|u(\cdot)\|_{L_{\infty}\left(\mathbb{R} ; H^{1}\right)}^{2}=\operatorname{ess} \sup \left\{\|u(s)\|^{2} \mid s \in \mathbb{R}\right\} \leqslant r^{-1}\|g\|^{2}, \quad \forall u \in \mathscr{K} .
$$


Tеорема 3.1. Траекторный аттрактор $\mathfrak{A}$ совпадает $с \Pi_{+} \mathscr{K}$ :

$$
\mathfrak{A}=\Pi_{+} \mathscr{K},
$$

где $\mathscr{K}$ - ядро уравнения (3.1) в пространстве $\mathscr{F}{ }^{\infty}$. Множество $\mathscr{K}$ ограничено в $\mathscr{F}^{\infty}$ и компактно в топологии $\Theta^{\mathrm{w}, \mathrm{loc}}$.

Теорема 3.1 доказывается аналогично теореме 2.2 (см. также [62]).

ЗАмЕЧАниЕ 3.3. Следующее вложение является компактным:

$$
\Theta_{+}^{\mathrm{w}, \text { loc }} \cap \mathscr{F}_{+}^{\text {loc }} \subseteq C^{\text {loc }}\left(\mathbb{R}_{+} ; H^{\delta}\right), \quad \forall \delta \in[0,1[
$$

(см. [20], [12]). Траекторный аттрактор $\mathfrak{A}=\Pi_{+} \mathscr{K}$ удовлетворяет (3.30), и, следовательно, для любого множества $\mathfrak{B} \in \mathscr{K}^{+}(N)$ имеет место сильный предел

$$
\operatorname{dist}_{C\left([0, M] ; H^{\delta}\right)}(T(t) \mathfrak{B}, \mathfrak{A}) \rightarrow 0 \quad(t \rightarrow+\infty),
$$

где $M-$ произвольное положительное число.

Отметим, что в формуле (3.35) мы не можем положить $\delta=1$. Это связано с тем, что построенный нами траекторный аттрактор $\mathfrak{A}$ притягивает траектории в слабой топологии $\Theta_{+}^{\mathrm{w}, \text { loc }}$ пространства $\mathscr{F}_{+}^{\text {loc }}$. Представляется естественным рассмотреть в $\mathscr{F}_{+}^{\text {loc }}$ также "сильную" топологию $\Theta_{+}^{\mathrm{s}, \text { loc }}$, которая вводится аналогично тому, как это сделано в разделе 2 для РД-системы. Однако доказать аналог теоремы 2.3 о притяжении к траекторному аттрактору $\mathfrak{A}$ в топологии $\Theta_{+}^{\mathrm{s}, \mathrm{loc}}$ без дополнительных предположений не удается. Это можно сделать, если допустить, что у внешней силы вихрь $\nabla \times g$ принадлежит $L_{\infty}\left(\mathbb{T}^{2}\right)$. В этом случае, сузив пространство траекторий $\mathscr{K}^{+}(N)$, можно построить траекторный аттрактор в сильной топологии $\Theta_{+}^{\mathrm{s}, \mathrm{loc}}$ (см. [63]). При доказательстве притяжения в сильной топологии используется техника Юдовича (см. [59], [60]), с помощью которой устанавливается, что траекторный аттрактор ограничен в пространстве $L_{\infty}\left(\mathbb{T}^{2} \times \mathbb{R}_{+}\right)$и на нем выполнена теорема единственности. Тогда можно воспользоваться методом энергетических тождеств, аналогично тому, как это делалось при доказательстве теоремы 2.3. Однако подчеркнем, что в рассмотренном выше более общем случае, когда $\nabla \times g \in L_{2}\left(\mathbb{T}^{2}\right)$, вопрос остается открытым, и мы сформулируем его в виде гипотезы.

ГиПотезА 3.1. Траекторный аттрактор $\mathfrak{A}$, построенный в теореме 3.1 , компактен в $\Theta_{+}^{\mathrm{s}, \text { loc }}$ и обладает свойством притяжения в сильной топологии $\Theta_{+}^{\mathrm{s}, \mathrm{loc}}$.

Построим глобальный аттрактор для уравнения (3.1) аналогично тому, как это было сделано в разделе 2 для системы реакции-диффузии.

Tеорема 3.2. Множество $\mathscr{A}:=\mathfrak{A}(0) \subset H^{1}$ является (слабым) глобалъным аттрактором системъ Эйлера с диссипацией (3.1), точнее, множество $\mathscr{A}$ ограничено и замкнуто в $H^{1}$. Кроме того,

1) для любого $N>0$ и для любого множества $\mathfrak{B} \subset \mathscr{K}^{+}(N)$ его сечение $\mathfrak{B}(t)=\{u(t) \mid u \in \mathfrak{B}\} \subset H^{1}$ стремится при $t \rightarrow+\infty \kappa \mathscr{A}$ в следующем смисле:

$$
\operatorname{dist}_{H^{\delta}}(\mathfrak{B}(t), \mathscr{A}) \rightarrow 0 \quad(t \rightarrow+\infty), \quad \forall \mathfrak{B} \subset \mathscr{K}^{+}(N), \quad \forall \delta \in[0,1) ;
$$


2) $\mathscr{A}$ является минимальным ограниченным и замкнутым множеством в $H^{1}$, которое обладает свойством притяжения (3.36).

Отметим, что доказать притяжение (3.36) в пространстве $H^{1}$ удается при дополнительном ограничении, что вихрь внешней силы $\nabla \times g$ принадлежит $L_{\infty}\left(\mathbb{T}^{2}\right)$ (см. [63]). В более общем случае задача остается открытой.

ГиПотезА 3.2. Множество $\mathscr{A}$ компактно в $H^{1}$, а свойство притяжения (3.36) справедливо и при $\delta=1$.

В следующем разделе будет изложена общая схема построения траекторного аттрактора эволюционного уравнения, записанного в форме абстрактного ОДУ в банаховом пространстве. Основное внимание будет уделено тем свойствам, которые следует проверять при построении траекторных аттракторов конкретных уравнений математической физики, не обязательно обладающих свойством единственности решений задачи Коши.

\section{4. Общая схема построения траекторного аттрактора эволюционного уравнения}

Пространство траекторий эволюционного уравнения. Рассматривается автономное уравнение вида

$$
\partial_{t} u=-F(u)
$$

где $F(u)$ - некоторый (нелинейный) дифференциальный или абстрактный оператор, действующий в определенных функциональных пространствах. Например, $F(\cdot): E \rightarrow E_{0}$, где $E$ и $E_{0}$ - банаховы пространства, причем предполагается, что $E \subset E_{0}$ (такое включение типично для дифференциальных операторов). К виду (4.1) принадлежат системы дифференциальных уравнений, которые рассматривались в разделах 2, 3. Например, для диссипативной системы Эйлера (3.1) следует положить $E=H$ и $E_{0}=H^{-1}$.

Мы будем изучать глобальные (по времени) решения $u(t), t \in \mathbb{R}_{+}$, уравнения (4.1), определенные на полуоси. Некоторое непустое и достаточно широкое семейство таких решений $\mathscr{K}^{+}$будет называться пространством траекторий уравнения (4.1). При изучении конкретных уравнений выбор пространства траекторий $\mathscr{K}^{+}$является исключительно ответственным шагом (см. разделы 1-3 настоящей главы II).

Сначала вводится функциональное пространство $\mathscr{F}_{+}^{\text {loc }}$, состоящее из функций $f(t), t \in \mathbb{R}_{+}$, со значениями в пространстве $E$, в котором будет находиться пространство траекторий уравнения (4.1). При этом функции $f(t)$ из $\mathscr{F}_{+}^{\text {loc }}$, рассматриваемые на интервале $\{0 \leqslant t \leqslant M\}$ при каждом $M>0$, принадлежат пространству $\mathscr{F}(0, M ; E)$, которое является банаховым.

Например, $\mathscr{F}(0, M ; E)=C([0, M] ; E)$ - пространство непрерывных функций со значениями в $E$ (в разделе 1 пространство $E$ равно $\left.\mathbb{R}^{N}\right)$. Другие примеры: $\mathscr{F}(0, M ; E)=L_{p}(0, M ; E)$, где $1 \leqslant p<+\infty$, и $\mathscr{F}(0, M ; E)=L_{\infty}(0, M ; E)$. Некоторые примеры получаются с помощью различных комбинаций (пересечение, 
прямое произведение и т. п.) подобных пространств. В разделе 2 при изучении системы реакции-диффузии рассматривалось пространство

$$
\mathscr{F}(0, M ; E)=L_{\infty}(0, M ; H) \cap L_{2}(0, M ; V) \cap L_{\mathbf{p}}\left(0, M ; L_{\mathbf{p}}(\Omega)\right) \cap\left\{\partial_{t} v \in \mathscr{L}(0, M)\right\},
$$

а для диссипативной системе Эйлера (раздел 3)

$$
\mathscr{F}(0, M ; E)=L_{\infty}(0, M ; H) \cap\left\{u(\cdot) \mid \partial_{t} u(\cdot) \in L_{\infty}\left(0, M ; H^{-1}\right)\right\} .
$$

Относительно оператора $F(\cdot)$ в уравнении (4.1) предполагается, что если функция $f(\cdot)$ принадлежит пространству $\mathscr{F}(0, M ; E)$, то на любом интервале $[0, M]$ функция $F(f(t)), 0 \leqslant t \leqslant M$, принадлежит пространству $\mathscr{F}\left(0, M ; E_{0}\right)$, являющемуся некоторым расширением $\mathscr{F}(0, M ; E): \mathscr{F}(0, M ; E) \subseteq \mathscr{F}\left(0, M ; E_{0}\right)$. Производная по времени $\partial_{t} f(t), 0 \leqslant t \leqslant M$, понимается в обобщенном смысле, т. е. она считается обобщенной функцией со значениями в пространстве $E_{0}$, $\partial_{t} f \in \mathscr{D}^{\prime}\left(0, M ; E_{0}\right)$ (см. [20]). Предполагается, что $\mathscr{F}\left(0, M ; E_{0}\right) \subset \mathscr{D}^{\prime}\left(0, M ; E_{0}\right)$.

В рассмотренных примерах для системы реакции-диффузии $\mathscr{F}\left(0, M ; E_{0}\right)=$ $L_{\mathbf{q}}\left(0, M ; H^{-\mathbf{r}}(\Omega)\right)$, а для системы Эйлера $\mathscr{F}\left(0, M ; E_{0}\right)=L_{\infty}\left(0, M ; H^{-1}\right)$.

Функция $u(\cdot) \in \mathscr{F}(0, M ; E)$ называется $(4.1)$ в пространстве $\mathscr{F}(0, M ; E)$ на интервале $[0, M]$, если справедливо равенство $\partial_{t} u(t)=-F(u(t))$ в пространстве обобщенных функций $\mathscr{D}^{\prime}\left(0, M ; E_{0}\right)$.

Определим пространство

$$
\mathscr{F}_{+}^{\text {loc }}:=\left\{f(t), t \in \mathbb{R}_{+} \mid \Pi_{0, M} f(\cdot) \in \mathscr{F}(0, M ; E), \forall M>0\right\},
$$

где $\Pi_{0, M}$ обозначает оператор сужения на интервал $[0, M]$. В приведенных выше примерах, если $\mathscr{F}(0, M ; E)=C([0, M] ; E)$, то $\mathscr{F}_{+}^{\text {loc }}=C^{\text {loc }}\left(\mathbb{R}_{+} ; E\right)$, а если $\mathscr{F}(0, M ; E)=L_{p}(0, M ; E)$, то $\mathscr{F}_{+}^{\text {loc }}=L_{p}^{\text {loc }}\left(\mathbb{R}_{+} ; E\right)$. Для РД-системы простран-

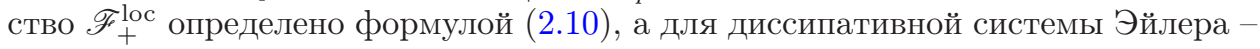
формулой (3.23).

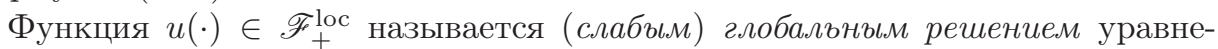
ния (4.1) в пространстве $\mathscr{F}_{+}^{\text {loc }}$, если при любом $M>0$ функция $\Pi_{0, M} u(t)$ принадлежит $\mathscr{F}(0, M ; E)$ и является $(4.1)$ на интервале $[0, M]$.

Через $\mathscr{K}^{+}$обозначается некоторое множество глобальных решений (4.1) из пространства $\mathscr{F}_{+}^{\text {loc }}$. Отметим, что $\mathscr{K}^{+}$не обязательно является множеством всех решений этого уравнения из $\mathscr{F}_{+}^{\text {loc }}$ (см. раздел 3$)$. Элементы $\mathscr{K}^{+}$будут называться траекториями, а множество $\mathscr{K}^{+}-$пространством траекторий уравнения (4.1).

В дальнейшем переменную времени в любом слабом решении $u(\cdot)$ уравнения (4.1) мы будем обозначать $s=t$ и писать $u(s), s \geqslant 0$, вместо $u(t), t \geqslant 0$. Элементы пространства $\mathscr{F}_{+}^{\text {loc }}$ будем также обозначать $f(s), s \geqslant 0$.

Рассмотрим трансляционные операторы $T(h), h \geqslant 0$, которые действуют в пространстве $\mathscr{F}_{+}^{\text {loc }}$ по формуле

$$
T(h) f(s)=f(h+s), \quad \forall h \geqslant 0 .
$$

Естественным образом предполагается, что оператор $T(h)$ непрерывно отображает пространство $\mathscr{F}(0, M ; E)$ в $\mathscr{F}(0, M-h ; E)$ при $h<M$. Следовательно, $T(h)$ переводит $\mathscr{F}_{+}^{\text {loc }}$ в себя при любом $h \geqslant 0$. 
Легко видеть, что семейство операторов $\{T(h), h \geqslant 0\}=:\{T(h)\}$ образует полугруппу на пространстве $\mathscr{F}_{+}^{\text {loc }}$

$$
T(0)=\mathrm{Id}, \quad T\left(h_{1}\right) T\left(h_{2}\right)=T\left(h_{1}+h_{2}\right), \quad \forall h_{1}, h_{2} \geqslant 0 .
$$

Заменим переменную $h$ на переменную времени $t$ и рассмотрим действие полугруппы $\{T(t)\}$ на $\mathscr{K}^{+}$. Предполагается, что пространство $\mathscr{K}^{+}$обладает следующим свойством:

$$
T(t) \mathscr{K}^{+} \subseteq \mathscr{K}^{+}, \quad \forall t \geqslant 0 .
$$

Иначе говоря, если $u(s), s \in \mathbb{R}_{+}$, является глобальным решением (4.1) из $\mathscr{K}^{+}$, то при любом $h \geqslant 0$ функция $u(h+s), s \in \mathbb{R}_{+}$, также является глобальным решением (4.1) из $\mathscr{K}^{+}$. Такое свойство весьма естественно для решений общих автономных уравнений, рассматриваемых в этом разделе. Различные пространства траекторий были рассмотрены в разделах 1-3.

Далее изучаются свойства притяжения семейств траекторий из $\mathscr{K}^{+}$под действием полугруппы $\{T(t)\}$ при $t \rightarrow+\infty$.

Траекторный аттрактор. Зададим топологию $\Theta_{+}^{\text {loc }}$ в пространстве $\mathscr{F}_{+}^{\text {loc }}$. Через $\Theta(0, M ; E)$ обозначим некоторую топологию в пространстве $\mathscr{F}(0, M ; E)$, которая для простоты определяется через понятие сходимости последовательностей в этом пространстве. Такой топологией может быть сильная, слабая, *-слабая или иная топология сходимости в банаховом пространстве $\mathscr{F}(0, M ; E)$.

Обозначим через $\Theta_{+}^{\text {loc }}$ топологию в $\mathscr{F}_{+}^{\text {loc }}$, которая порождается локальной сходимостью в $\Theta(0, M ; E)$ при каждом $M>0$. Точнее, по определению, последовательность $\left\{f_{m}(\cdot), m \in \mathbb{N}\right\}$ из $\mathscr{F}_{+}^{\text {loc }}$ сходится к элементу $f(\cdot) \in \mathscr{F}_{+}^{\text {loc }}$ в топологии $\Theta_{+}^{\text {loc }}$ при $m \rightarrow \infty$, если при любом $M>0$ последовательность $\left\{\Pi_{0, M} f_{m}(\cdot)\right\}$ из $\mathscr{F}(0, M ; E)$ сходится к $\Pi_{0, M} f(\cdot) \in \mathscr{F}(0, M ; E)$ в топологии $\Theta(0, M ; E)$ при $m \rightarrow \infty$. Определенная таким образом топология $\Theta_{+}^{\text {loc }}$ называется индуктивным пределом топологий $\Theta(0, M ; E)$ при $M \rightarrow+\infty$.

Например, если $\mathscr{F}(0, M ; E)=C([0, M] ; E)$ и топология $\Theta(0, M ; E)$ порождена равномерной (по $s \in[0, M])$ сильной сходимостью по норме $E$, то $\Theta_{+}^{\text {loc }}-$ топология равномерной сильной сходимости в пространстве $\mathscr{F}_{+}^{\text {loc }}=C^{\text {loc }}\left(\mathbb{R}_{+} ; E\right)$ на каждом конечном отрезке (см. раздел 1, где $\left.E=\mathbb{R}^{N}\right)$. Пусть $\mathscr{F}(0, M ; E)=$ $L_{p}(0, M ; E)$, причем $p>1$ и пространство $E$ рефлексивно. Зададим топологию $\Theta(0, M ; E):=L_{p, \mathrm{w}}(0, M ; E)$ слабой сходимости этого банахова пространства. В этом случае $\Theta_{+}^{\text {loc }}$ - топология слабой локальной сходимости в $L_{p, \mathrm{w}}(0, M ; E)$

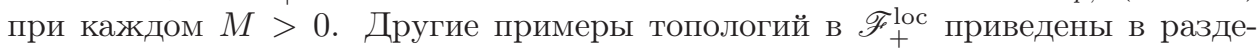
лах 2, 3 (см. также следующие разделы).

Мы будем предполагать, что топологическое пространство $\mathscr{F}_{+}^{\text {loc }} \cap \Theta_{+}^{\text {loc }}$ является хаусдорфовым, т. е. любые две точки в нем можно разделить непересекающимися окрестностями. В конкретных приложениях это свойство всегда выполняется, например если известно, что топология $\Theta_{+}^{\text {loc }}$ является метризуемой. Отметим, что неметризуемые топологии также иногда используются при изучении траекторных аттракторов.

Заметим, что трансляционная полугруппа $\{T(t)\}$ непрерывна на $\mathscr{F}_{+}^{\text {loc }}$ в топологии $\Theta_{+}^{\text {loc }}$. Это прямо вытекает из определения топологии индуктивного 
предела (доказательство аналогично доказательству утверждения 1.2). Это важное свойство позволяет использовать общую конструкцию глобальных аттракторов для непрерывных полугрупп.

Определим также банахово пространство

$$
\mathscr{F}_{+}^{\mathrm{b}}:=\left\{f(\cdot) \in \mathscr{F}_{+}^{\mathrm{loc}} \mid\|f\|_{\mathscr{F}_{+}^{\mathrm{b}}}<\infty\right\},
$$

где норма в $\mathscr{F}_{+}^{\mathrm{b}}$ задается формулой

$$
\|f\|_{\mathscr{F}+}:=\sup _{h \geqslant 0}\left\|\Pi_{0,1} f(s+h)\right\|_{\mathscr{F}(0,1 ; E)} .
$$

Пространство $\mathscr{F}_{+}^{\mathrm{b}}$ необходимо для задания класса ограниченных множеств в $\mathscr{K}^{+}$. В рассмотренных выше примерах, если $\mathscr{F}(0, M ; E)=C([0, M] ; E)$, то $\mathscr{F}_{+}^{\mathrm{b}}=C_{\mathrm{b}}\left(\mathbb{R}_{+} ; E\right)$ - пространство непрерывных ограниченных функций с равномерной нормой

$$
\|f\|_{C_{\mathrm{b}}\left(\mathbb{R}_{+} ; E\right)}:=\sup _{s \geqslant 0}\|f(s)\|_{E} .
$$

В случае $\mathscr{F}(0, M ; E)=L_{p}(0, M ; E)$ норма в пространстве $\mathscr{F}_{+}^{\mathrm{b}}=L_{p}^{\mathrm{b}}\left(\mathbb{R}_{+} ; E\right)$ задается формулой

$$
\|f\|_{L_{p}^{\mathrm{b}}\left(\mathbb{R}_{+} ; E\right)}:=\sup _{h \geqslant 0}\left(\int_{h}^{h+1}\|f(s)\|_{E}^{p} d s\right)^{1 / p} .
$$

Для РД-системы норма в соответствующем пространстве $\mathscr{F}_{+}^{\mathrm{b}}($ см. (2.11)) определена формулой (2.12), а для системы Эйлера норма в пространстве (3.24) определена формулой (3.25).

В дальнейшем предполагается, что $\mathscr{K}^{+} \subset \mathscr{F}_{+}^{\mathrm{b}}$, т. е. любое решение $u(s)$, $s \geqslant 0$, уравнения (4.1) из пространства траекторий $\mathscr{K}^{+}$имеет конечную норму (4.3). Для системы реакции-диффузии это свойство установлено в утверждении 2.4, а для системы Эйлера сами пространства $\mathscr{K}^{+}(N)$ ограничены В $\mathscr{F}_{+}^{\infty}$.

Приведем определение траекторного аттрактора полугруппы $\{T(t)\}$, действующей на $\mathscr{K}^{+}$. Для этого обобщим понятие притягивающего множества.

Множество $\mathscr{P} \subseteq \mathscr{K}^{+}$называется притягивающим для полугруппы $\{T(t)\}$ в топологии $\Theta_{+}^{\text {loc }}$, если для любого множества $\mathfrak{B} \subset \mathscr{K}^{+}$, ограниченного по норме $\mathscr{F}_{+}^{\mathrm{b}}$, множество $T(t) \mathfrak{B}$ притягивается к $\mathscr{P}$ в топологии $\Theta_{+}^{\text {loc }}$ при $t \rightarrow+\infty$, т. е. для любой окрестности $\mathscr{O}(\mathscr{P})$ множества $\mathscr{P}$ в топологии $\Theta_{+}^{\text {loc }}$ найдется $\tau=\tau(\mathfrak{B}, \mathscr{O}) \geqslant 0$ такое, что $T(t) \mathfrak{B} \subseteq \mathscr{O}(\mathscr{P})$ при всех $t \geqslant \tau$.

Часто удается построить поглощающее множество полугруппы $\{T(t)\}$. Множество $\mathscr{P} \subseteq \mathscr{K}^{+}$называется поглощающим для полугруппы $\{T(t)\}$, если для любого множества $\mathfrak{B} \subset \mathscr{K}^{+}$, ограниченного в $\mathscr{F}_{+}^{\mathrm{b}}$, найдется $\tau=\tau(\mathfrak{B}) \geqslant 0$ такое, что $T(t) \mathfrak{B} \subseteq \mathscr{P}$ при всех $t \geqslant \tau$.

ОПРеДЕЛЕНИЕ 4.1. Множество $\mathfrak{A} \subseteq \mathscr{K}^{+}$называется траекторным атmрактором полугруппы $\{T(t)\}$ на $\mathscr{K}^{+}$в топологии $\Theta_{+}^{\text {loc }}$, если:

1) множество $\mathfrak{A}$ ограничено в $\mathscr{F}_{+}^{\mathrm{b}}$ и компактно в топологии $\Theta_{+}^{\text {loc; }}$

2) $\mathfrak{A}$ является притягивающим множеством в топологии $\Theta_{+}^{\text {loc }}$;

3) $\mathfrak{A}$ строго инвариантно: $T(t) \mathfrak{A}=\mathfrak{A}$ при любом $t \geqslant 0$. 
Легко устанавливается, что если траекторный аттрактор существует, то он является единственным.

Оказывается, что траекторный аттрактор существует, если у полугруппы $\{T(t)\}$ имеется притягивающее множество, которое ограниченно по норме $\mathscr{F}_{+}^{\mathrm{b}}$ и которое является компактным множеством в топологии $\Theta_{+}^{\text {loc }}$. Такие притягивающие (и даже поглощающие) множества были построены для конкретных уравнений в разделах $1-3$.

Сформулируем общую теорему о существовании траекторного аттрактора.

Теорема 4.1. Пусть пространство траекторий $\mathscr{K}^{+}$, соответствующее уравнению (4.1), принадлежит пространству $\mathscr{F}_{+}^{\mathrm{b}}$, причем выполнено (4.2). Предположим, что у полугруппы $\{T(t)\}$ имеется притягивающее множество $\mathscr{P}$, которое ограничено по норме $\mathscr{F}_{+}^{\mathrm{b}}$ и компактно в топологии $\Theta_{+}^{\mathrm{loc}}$. Тогда трансляиионная полугруппа $\{T(t)\}$ имеет траекторный аттрактор $\mathfrak{A} \subseteq \mathscr{P}$. Множество $\mathfrak{A}$ ограничено в $\mathscr{F}_{+}^{\mathrm{b}}$ и компактно в $\Theta_{+}^{\text {loc }}$.

Доказательство этой общей теоремы приведено в [12]. В тех (весьма частых) случаях, когда для полугруппы $\{T(t)\}$ удается построить поглощающее множество $\mathscr{P}$, которое ограничено в $\mathscr{F}_{+}^{\mathrm{b}}$, компактно в $\Theta_{+}^{\mathrm{loc}}$, и при этом топология $\Theta_{+}^{\text {loc }}$ является метризуемой на $\mathscr{P}$, теорему 4.1 легко вывести из общей теоремы 1.1 главы I о глобальных аттракторах непрерывных полугрупп в полных метрических пространствах. В этом случае траекторный аттрактор строится по формуле

$$
\mathfrak{A}=\bigcap_{t \geqslant 0} T(t) \mathscr{P} .
$$

По этой схеме были построены траекторные аттракторы уравнений, которые изучались в разделах 1-3.

Ядро эволюционного уравнения. Дадим описание траекторного аттрактора $\mathfrak{A}$ уравнения (4.1), используя понятие ядра этого уравнения. Рассмотрим уравнение (4.1) на всей оси времени:

$$
\partial_{t} u=-F(u), \quad t \in \mathbb{R} .
$$

Выше было введено пространство траекторий (решений) $\mathscr{K}^{+}$этого уравнения на полуоси $\mathbb{R}_{+}$. Распространим это определение на всю ось $\mathbb{R}$.

Наряду с $\mathscr{F}(0, M ; E)$ рассматриваются пространства $\mathscr{F}(-M, M ; E)$, состоящие из функций $f(t),-M \leqslant t \leqslant M$, со значениями в пространстве $E$. Пространства $\mathscr{F}(-M, M ; E)$ также являются банаховыми. Затем рассматриваются слабые решения $u(t),-M \leqslant t \leqslant M$, уравнения $(4.4)$ из $\mathscr{F}(-M, M ; E)$, которые удовлетворяют уравнению в смысле пространства обобщенных функций $\mathscr{D}^{\prime}\left(-M, M ; E_{0}\right)$, причем $\partial_{t} u \in \mathscr{F}\left(-M, M ; E_{0}\right) \subset \mathscr{D}^{\prime}\left(-M, M ; E_{0}\right)$.

Аналогично $\mathscr{F}_{+}^{\text {loc }}$ и $\mathscr{F}_{+}^{\mathrm{b}}$ вводятся пространства $\mathscr{F}^{\mathrm{loc}}$ и $\mathscr{F}^{\mathrm{b}}$ :

$$
\begin{aligned}
\mathscr{F}^{\text {loc }} & :=\left\{f(s), s \in \mathbb{R} \mid \Pi_{-M, M} f(\cdot) \in \mathscr{F}(-M, M ; E), \forall M>0\right\}, \\
\mathscr{F}^{\mathrm{b}} & :=\left\{f(\cdot) \in \mathscr{F}^{\mathrm{loc}} \mid\|f\|_{\mathscr{F} \mathrm{b}}<\infty\right\} .
\end{aligned}
$$

Норма в $\mathscr{F}^{\mathrm{b}}$ задается формулой

$$
\|f\|_{\mathscr{F} \mathrm{b}}:=\sup _{h \in \mathbb{R}}\left\|\Pi_{0,1} f(s+h)\right\|_{\mathscr{F}(0,1 ; E)} .
$$


Аналогично $\Theta_{+}^{\text {loc }}$ вводится топология $\Theta^{\text {loc }}$, которая является индуктивным пределом соответствующих топологий $\Theta(-M, M ; E)$ при $M \rightarrow+\infty$.

На функциях из $\mathscr{F}^{\text {loc }}$ действуют операторы трансляции $\{T(h), h \in \mathbb{R}\}$ :

$$
T(h) f(s)=f(s+h), \quad s \in \mathbb{R}, \quad \forall h \in \mathbb{R},
$$

которые, очевидно, образуют группу в $\mathscr{F}^{\text {loc }}$.

Функция $u(s), s \in \mathbb{R}$, со значениями в $E$ называется полным решением уравнения (4.4) в пространстве $\mathscr{F}^{\text {loc }}$, если $u(\cdot) \in \mathscr{F}^{\text {loc }}$ и при всех $M>0$ функция $\Pi_{-M, M} u(s)$ является решением этого уравнения из $\mathscr{F}(-M, M ; E)$ в пространстве обобщенных функций $\mathscr{D}^{\prime}\left(-M, M ; E_{0}\right)$. Оператор ограничения на полуось $\mathbb{R}_{+}$обозначается, как обычно, через $\Pi_{+}$.

Пусть задано некоторое пространство траекторий $\mathscr{K}^{+}$уравнения (4.1).

ОПРЕДЕлЕниЕ 4.2. Ядро $\mathscr{K}$ уравнения (4.1) есть объединение всех полных решений $u(s), s \in \mathbb{R}$, этого уравнения из $\mathscr{F}^{\mathrm{loc}}$, для которых $\Pi_{+} T(h) u \in \mathscr{K}^{+}$ при любом $h \in \mathbb{R}$ и которые принадлежат $\mathscr{F}$, т. е.

$$
\left\|\Pi_{0,1} u(s+h)\right\|_{\mathscr{F}(0,1 ; E)} \leqslant C_{u}, \quad \forall h \in \mathbb{R} .
$$

Заметим, что

$$
T(h) \mathscr{K}=\mathscr{K}, \quad \forall h \in \mathbb{R} .
$$

Кроме того, легко видеть, что множество $\Pi_{+} \mathscr{K} \subset \mathscr{K}^{+}$ограничено в $\mathscr{F}_{+}^{\mathrm{b}}$ и строго инвариантно относительно полугруппы трансляций $\{T(h), h \geqslant 0\}$ :

$$
T(h) \Pi_{+} \mathscr{K}=\Pi_{+} \mathscr{K}, \quad \forall h \geqslant 0 .
$$

Следовательно, множество $\Pi_{+} \mathscr{K}$ принадлежит траекторному аттрактору уравнения (4.1): $\Pi_{+} \mathscr{K} \subseteq \mathfrak{A}$. Обратное включение также имеет место, и в этом заключается основное содержание следующей теоремы.

ТеОрема 4.2. Пусть выполнены условия теоремы 4.1. Тогда $\mathfrak{A}=\Pi_{+} \mathscr{K}$, причем ядро $\mathscr{K}$ ограничено в $\mathscr{F}^{\mathrm{b}}$ и компактно в $\Theta^{\mathrm{loc}}$.

Доказательство приведено в [12]. Оно аналогично доказательству теоремы 2.2.

Поясним характер притяжения любого ограниченного в $\mathscr{F}_{+}^{\mathrm{b}}$ множества траекторий $\mathfrak{B}$ из $\mathscr{K}^{+}$под действием трансляций $T(t)$ к траекторному аттрактору $\mathfrak{A}$ при $t \rightarrow+\infty$.

СледСтвиЕ 4.1. Пусть выполнены условия теоремы 4.1. Рассмотрим любое ограниченное в $\mathscr{F}_{+}^{\mathrm{b}}$ множество траекторий $\mathfrak{B}$ из $\mathscr{K}^{+}$. Тогда при любом $M>0$ множество $\Pi_{0, M} T(t) \mathfrak{B}$ стремится $\kappa \Pi_{0, M} \mathfrak{A}=\Pi_{0, M} \mathscr{K}$ в топологии $\Theta(0, M ; E)$ при $t \rightarrow+\infty$. Например, если топология $\Theta(0, M ; E)$ метризуема (скажем, на достаточно большом шаре в $\mathscr{F}_{+}^{\mathrm{b}}$, содержащем $\left.\mathfrak{B}\right)$, то

$$
\operatorname{dist}_{\Theta(0, M ; E)}\left(\Pi_{0, M} T(t) \mathfrak{B}, \Pi_{0, M} \mathscr{K}\right) \rightarrow 0 \quad(t \rightarrow+\infty),
$$

где $\operatorname{dist}_{Y}(\cdot, \cdot)$ обозначает отклонение по Хаусдорфу множеств в метрике $Y$. 
В приложениях к конкретным уравнениям, когда требуется построить притягивающее или поглощающее множество с нужными свойствами, таким множеством часто служит $\mathscr{P}=\mathscr{B}_{R} \cap \mathscr{K}^{+}$, где $\mathscr{B}_{R}-$ шар в пространстве $\mathscr{F}_{+}^{\mathrm{b}}$ достаточно большого радиуса $R: \mathscr{B}_{R}=\left\{f \in \mathscr{F}_{+}^{\mathrm{b}} \mid\|f\|_{\mathscr{F}_{+}^{\mathrm{b}}} \leqslant R\right\}, R \gg 1$. Свойство поглощения (и притяжения) к такому множеству обычно следует из неравенства вида

$$
\|T(t) u\|_{\mathscr{F}_{+}^{\mathrm{b}}} \leqslant C\left(\|u\|_{\mathscr{F}_{+}^{\mathrm{b}}}\right) \mathrm{e}^{-\gamma t}+R_{0}
$$

при любом $u(\cdot) \in \mathscr{K}^{+}$и всех $t \geqslant 0$, где $C(R)$ зависит от $R$, а $R_{0}>0$ и $\gamma>0$ не зависят от $u(\cdot)$. Как правило, неравенство (4.8) удается получить из априорных оценок для решений уравнения (4.1), которые используются при доказательстве существования этих решений. Если дополнительно известно, что шары $\mathscr{B}_{R}$ компактны в топологии $\Theta_{+}^{\text {loc }}$, то множество $\mathscr{P}=\mathscr{B}_{2 R_{0}} \cap \mathscr{K}^{+}$удовлетворяет всем условиям теоремы 4.1 .

Обобщение глобального аттрактора. В предыдущих разделах для конкретных уравнений после траекторных аттракторов строились также (обобщенные) глобальные аттракторы. Это возможно сделать и для абстрактного эволюционного уравнения (4.1). Проще всего бывает построить слабый глобальный аттрактор.

Мы будем предполагать, что любая траектория $u(s), s \geqslant 0$, уравнения (4.1) из рассматриваемого пространства траекторий $\mathscr{K}^{+}$обладает тем свойством, что $u(\cdot) \in L_{\infty}\left(\mathbb{R}_{+} ; E\right)$. Кроме того, предполагается, что функция $u(s), s \geqslant 0$, является слабо непрерывной со значениями в банаховом пространстве $E, u(\cdot) \in$ $C_{\mathrm{w}}\left(\mathbb{R}_{+} ; E\right)$, т. е. для любого функционала $\phi \in E^{*}$ скалярная функция $\langle\phi, u(s)\rangle$ непрерывна при $s \geqslant 0$. В силу леммы 2.1 (Лионс-Мадженес) для этого достаточно, чтобы траектория $u(s), s \geqslant 0$, принадлежала $L_{\infty}\left(\mathbb{R}_{+} ; E\right)$ и была слабо (или сильно) непрерывной функцией со значениями в некотором более широком банаховом пространстве $E_{0}, E \subseteq E_{0}$. Последнее условие часто выполнено для обобщенных решений конкретных эволюционных уравнений. При выполнении этих предположений для любой траектории $u \in \mathscr{K}^{+}$ее значения $u(s)$ принадлежат $E$ при всех $s \geqslant 0$ и при этом справедливо неравенство

$$
\|u(s)\|_{E} \leqslant\|u(\cdot)\|_{C_{\infty}\left(\mathbb{R}_{+} ; E\right)}, \quad \forall s \geqslant 0 .
$$

Предположим, что $\mathscr{F}_{+}^{\mathrm{b}} \subset L_{\infty}\left(\mathbb{R}_{+} ; E\right)$, а топология $\Theta_{+}^{\text {lос }}$ сильнее топологии пространства $C_{\mathrm{w}}\left(\mathbb{R}_{+} ; E\right)$.

Рассмотрим произвольное множество траекторий $\mathfrak{B} \subset \mathscr{K}^{+}$. Обозначим через $\mathfrak{B}(t)$ сечение множества $\mathfrak{B}$ в момент времени $t$ :

$$
\mathfrak{B}(t):=\{u(t) \mid u \in \mathfrak{B}\} \subset E .
$$

Множества $\mathfrak{B}(t)$ равномерно ограничены в $E$ :

$$
\|\mathfrak{B}(t)\|_{E}:=\sup _{u \in \mathfrak{B}(t)}\|u\|_{E} \leqslant\|\mathfrak{B}\|_{C_{\infty}\left(\mathbb{R}_{+} ; E\right)} .
$$

Пусть $\mathfrak{A}$ - траекторный аттрактор уравнения (4.1). Тогда множество $\mathfrak{A}$ ограничено в $L_{\infty}\left(\mathbb{R}_{+} ; E\right)$ и компактно в $C_{\mathrm{w}}\left(\mathbb{R}_{+} ; E\right)$. Рассмотрим сечение множества $\mathfrak{A}$ 
в любой момент $t$ :

$$
\mathfrak{A}(t)=\{u(t) \mid u \in \mathfrak{A}\} \subset E .
$$

Из свойства строгой инвариантности траекторного аттрактора $\mathfrak{A}$ под действием трансляционной полугруппы $\{T(t\}$ вытекает, что

$$
\mathfrak{A}(t)=\mathfrak{A}(0), \quad \forall t \geqslant 0 .
$$

Кроме того, поскольку $\mathfrak{A}$ ограничено в $L_{\infty}\left(\mathbb{R}_{+} ; E\right)$ и компактно в $C_{\mathrm{w}}\left(\mathbb{R}_{+} ; E\right)$, то множество $\mathfrak{A}(0)$ ограничено в $E$, слабо замкнуто в $E$, а значит, сильно замкнуто в $E$. Отметим, что в силу теоремы 4.2 имеет место равенство $\mathfrak{A}(0)=\mathscr{K}(0)$, где $\mathscr{K}(0)$ - сечение ядра $\mathscr{K}$ уравнения (4.1) в пространстве $\mathscr{F}^{\mathrm{b}}$.

ОПредЕЛЕНИЕ 4.3. Множество $\mathscr{A} \subset E$ называется слабым глобалъным аттрактором уравнения (4.1), если оно ограничено и замкнуто в $E$, а кроме того, выполнены следующие свойства:

1) для любого множества $\mathfrak{B} \subset \mathscr{K}^{+}$, ограниченного по норме $\mathscr{F}_{+}^{\mathrm{b}}$, его сечение $\mathfrak{B}(t)$ притягивается к $\mathscr{A}$ в слабой топологии $E$ при $t \rightarrow+\infty$, т. е. для любой окрестности $\mathscr{O}_{\mathrm{w}}(\mathscr{A})$ множества $\mathscr{A}$ в слабой топологии $E$ найдется момент времени $\tau=\tau\left(\mathfrak{B}, \mathscr{O}_{\mathrm{w}}\right)$ такой, что

$$
\mathfrak{B}(t) \subset \mathscr{O}_{\mathrm{w}}(\mathscr{A}) \quad \text { при всех } t \geqslant \tau \text {; }
$$

2) $\mathscr{A}$ является минимальным ограниченным и замкнутым множеством в пространстве $E$, которое притягивает при $t \rightarrow+\infty$ сечения всех ограниченных множеств из $\mathscr{K}^{+}$в слабой топологии $E$.

Tеорема 4.3. Пусть $\mathscr{F}_{+}^{\mathrm{b}} \subset L_{\infty}\left(\mathbb{R}_{+} ; E\right)$ u $\Theta_{+}^{\text {loc }} \subset C_{\mathrm{w}}\left(\mathbb{R}_{+} ; E\right)$, u nycmъ въполнены условия теоремы 4.1. Тогда множество $\mathscr{A}:=\mathfrak{A}(0)=\mathscr{K}(0)$ является слабым глобальным аттрактором уравнения (4.1).

Доказательство приведено в [12] (см. также утверждение 5.3 ниже).

ЗАмЕчАниЕ 4.1. Аналогично определению 4.3 можно определить сильный глобальный аттрактор в пространстве $E$, если потребовать от множества $\mathscr{A}$ компактность в $E$ и строгое притяжение $\mathfrak{B}(t)$ к $\mathscr{A}$ при $t \rightarrow+\infty$ по норме $E$ для любого ограниченного множества траекторий $\mathfrak{B} \subset \mathscr{K}^{+}$. В конкретных приложениях бывает относительно легко построить слабый глобальный аттрактор, но доказательство того, что это множество является сильным глобальным аттрактором, может оказаться весьма сложной самостоятельной проблемой. Для РД-системы это сделать удается, а для 3D-системы Навье-Стокса это остается нерешенной проблемой (см. раздел 5).

В заключение покажем, как можно использовать теорему 4.3 для нахождения глобального аттрактора уравнения (4.1), для которого выполнена теорема единственности задачи Коши.

Предположим, что рассматриваемое пространство траекторий $\mathscr{K}^{+}$уравнения (4.1) обладает следующим свойством: для любого $u_{0} \in E$ существует и притом единственная траектория $u(\cdot) \in \mathscr{K}^{+}$такая, что

$$
u(0)=u_{0} .
$$


Тогда при выполнении условий теоремы 4.3 в пространстве $E$ можно построить полугруппу $\{S(t)\}$, отвечающую задаче Коши (4.1), (4.9):

$$
S(t) u_{0}=u(t), \quad t \geqslant 0 .
$$

При этом множество $\mathscr{A}:=\mathfrak{A}(0)=\mathscr{K}(0)$ будет являться слабым глобальным аттрактором полугруппы (4.1). В конкретных приложениях иногда можно доказать, что этот слабый глобальный аттрактор является сильным, т. е. он притягивает ограниченные множества в сильной топологии $E$.

В следующих разделах по изложенной выше схеме будут исследованы еще три важных уравнения математической физики, для которых теорема единственности решений задачи Коши не установлена, а именно, 3D-система НавьеСтокса, диссипативное волновое уравнение с нелинейной функцией взаимодействия, обладающей полиномиальным ростом произвольной степени, а также нелинейное эллиптическое уравнение в цилиндре.

\section{5. Траекторный аттрактор трехмерной системы Навье-Стокса}

Слабые решения системы Навье-Стокса. Рассматривается автономная $3 \mathrm{D}$-система Навье-Стокса в ограниченной области $\Omega \Subset \mathbb{R}^{3}$ с условиями прилипания на границе $\partial \Omega$ :

$$
\left\{\begin{array}{l}
\partial_{t} u+\sum_{j=1}^{3} u^{j} \partial_{x_{j}} u-\nu \Delta u=-\nabla p+g(x), \\
\sum_{j=1}^{3} \partial_{x_{j}} u^{j}=0,\left.\quad u\right|_{\partial \Omega}=0, \quad x=\left(x_{1}, x_{2}, x_{3}\right) \in \Omega, \quad t \geqslant 0 .
\end{array}\right.
$$

Неизвестными являются векторная функция $u=\left(u^{1}(x, t), u^{2}(x, t), u^{3}(x, t)\right)$ скорости и скалярная функция $p=p(x, t)$ давления жидкости в точке $x$ в момент времени $t$. Кинематическая вязкость $\nu>0$ и плотность внешней силы $g(x)=\left(g^{1}(x), g^{2}(x), g^{3}(x)\right)$, действующей на жидкость в точке $x \in \Omega$, считаются заданными. Стандартным образом, с помощью исключения давления, система (5.1) приводится к функциональному дифференциальному уравнению

$$
\partial_{t} u+\nu L u+B(u, u)=g(x), \quad(\nabla, u)=0,\left.\quad u\right|_{\partial \Omega}=0,
$$

где $u=u(x, t)$ - неизвестная вектор-функция, $L u=-P \Delta u$ - оператор Стокса,

$$
B(u, v)=P(\nabla, u) v=P \sum_{j=1}^{3} u^{j} \partial_{x_{j}} v, \quad(\nabla, u)=\sum_{j=1}^{3} \partial_{x_{j}} u^{j}
$$

(см. [20], [25], [26]) и через $P$ обозначен ортогональный проектор Лерэ, который определяется ниже. Обозначим через $H$ и $V$ гильбертовы пространства, которые получаются при замыкании соответственно по норме $\left[L_{2}(\Omega)\right]^{3}$ и $\left[H_{0}^{1}(\Omega)\right]^{3}$ линейного пространства вектор-функций

$$
\mathscr{V}_{0}=\left\{v \in\left[C_{0}^{\infty}(\Omega)\right]^{3} \mid(\nabla, v)=0\right\} .
$$


Тогда $P$ - это ортогональный проектор в пространстве $\left[H_{0}^{1}(\Omega)\right]^{3}$ на подпространство $H$. Напомним, что скалярное произведение и норма в пространстве $H$ задаются формулами

$$
(u, v):=\int_{\Omega}(u(x), v(x)) d x, \quad|u|:=(u, u)^{1 / 2} .
$$

Отметим, что $V=\left\{v \in\left[H_{0}^{1}(\Omega)\right]^{3} \mid(\nabla, v)=0\right\}$. Пусть $V^{\prime}=V^{*}$ - пространство, сопряженное к $V$. Для $v \in V^{\prime}$ выражение $(v, u)$ обозначает значение функционала $v$ на векторе $u \in V$. Если $v \in H$, то $(v, u)$ обозначает обычное скалярное произведение функций в $H$. Оператор Стокса $L$ осуществляет изоморфизм между пространствами $V$ и $V^{\prime}$. Скалярное произведение и норма в пространстве $V$ определяются по формулам

$$
((v, u)):=\sum_{i, j=1}^{3} \int_{\Omega}\left(\partial_{x_{j}} v_{i}(x), \partial_{x_{j}} u_{i}(x)\right) d x=(L v, u), \quad\|u\|:=((u, u))^{1 / 2} .
$$

Предполагается, что в уравнении (5.2) внешняя сила $g$ принадлежит $V^{\prime}$.

Известно, что квадратичный оператор $B(u, u)$ действует из $V$ в $V^{\prime}$ и удовлетворяет неравенству

$$
\|B(u, u)\|_{V^{\prime}} \leqslant c\|u\|^{3 / 2}|u|^{1 / 2}, \quad \forall u \in V .
$$

Здесь $c$ - некоторая универсальная константа, не зависящая от области $\Omega$ (см. [20], [25], [26]).

Пусть задана некоторая функция $u(x, t) \in L_{2}(0, M ; V) \cap L_{\infty}(0, M ; H)$. Тогда, очевидно,

$$
L u(x, t) \in L_{2}\left(0, M ; V^{\prime}\right)
$$

а из (5.4) вытекает, что

$$
B(u(x, t), u(x, t)) \in L_{4 / 3}\left(0, M ; V^{\prime}\right) .
$$

Функция $u(x, t)$ называется слабым решением уравнения (5.2), если она удовлетворяет этому уравнению в смысле пространства $\mathscr{D}^{\prime}\left(0, M ; V^{\prime}\right)$ обобщенных функций со значениями в $V^{\prime}$ (см. [20]). Тогда из свойств (5.5) и (5.6) вытекает, что обобщенная производная по времени $\partial_{t} u(x, t)$ принадлежит $L_{4 / 3}\left(0, M ; V^{\prime}\right)$.

Из леммы 2.1 следует, что функция $u(\cdot, t), t \in[0, M]$, является слабо непрерывной в пространстве $H$. Следовательно, значения $u(x, t)$ определены при всех $t \in[0, M]$, причем

$$
u(\cdot, t) \in H, \quad \forall t \in[0, M] .
$$

Изложим вкратце классическую схему метода Галёркина построения слабого решения $u(x, t)$ трехмерной системы Навье-Стокса (5.2), которое удовлетворяет начальному условию

$$
\left.u\right|_{t=0}=u_{0}(x),
$$

где $u_{0}(\cdot) \in H-$ произвольно заданная функция. Отметим, что свойство (5.7) придает смысл начальному условию (5.8) в задаче Коши для (5.2). 
Пусть $\left\{w_{j}(x)\right\}$ - некоторая линейно независимая система вектор-функций из $V$, которая образует базис в пространстве $H$. (Например, это может быть система собственных функций оператора Стокса L.) Приближение Галёркина $u_{m}(x, t)$ задачи $(5.2),(5.8)$ порядка $m$ ищется в виде конечной суммы

$$
u_{m}(x, t)=\sum_{j=1}^{m} a_{j, m}(t) w_{j}(x), \quad m \in \mathbb{N} .
$$

Вещественные функции $a_{j, m}(t), j=1, \ldots, m$, должны удовлетворять следующей системе ОДУ:

$$
\frac{d}{d t} u_{m}+\nu P_{m} L u_{m}+P_{m} B\left(u_{m}, u_{m}\right)=P_{m} g, \quad u_{m}(0)=P_{m} u_{0}
$$

где $P_{m}$ обозначает ортогональный проектор в $H$ на конечномерное подпространство, натянутое на векторы $w_{1}, \ldots, w_{m}$, которое, очевидно, принадлежит $V$. Отметим, что

$$
P_{m} u_{0} \rightarrow u_{0} \quad(m \rightarrow \infty) \text { сильно в } H,
$$

так как последовательность $\left\{w_{j}\right\}$ образует базис в $H$. Система (5.9) имеет и притом единственное решение $u_{m}(x, t)$ в классе функций $C^{1}\left(\left[0, \tau_{m}\right) ; V\right)$ на некотором максимальном интервале времени $\left[0, \tau_{m}\right)$. Можно показать, что $\tau_{m}=+\infty$. Это вытекает из основной энергетической оценки, о которой речь пойдет ниже.

Из классических работ Лерэ и Хопфа (см. [20], [64], [65]) вытекает, что найдутся подпоследовательность $\left\{m^{\prime}\right\}$ и функция

$$
u(\cdot) \in L_{2}^{\text {loc }}\left(\mathbb{R}_{+} ; V\right) \cap L_{\infty}^{\text {loc }}\left(\mathbb{R}_{+} ; H\right), \quad \partial_{t} u(\cdot) \in L_{4 / 3}^{\text {loc }}\left(\mathbb{R}_{+} ; V^{\prime}\right),
$$

такие, что $u_{m^{\prime}}(\cdot) \rightarrow u(\cdot)\left(m^{\prime} \rightarrow \infty\right)$ слабо в $L_{2}(0, M ; V), *$-слабо в $L_{\infty}(0, M ; H)$, $\partial_{t} u_{m^{\prime}}(\cdot) \rightarrow \partial_{t} u(\cdot),\left(m^{\prime} \rightarrow \infty\right)$ слабо в $L_{4 / 3}\left(0, M ; V^{\prime}\right)$ при любом $M>0$ и при этом функция $u(\cdot)$ является слабым решением уравнения (5.2), удовлетворяющим начальному условию (5.8) (см., например, [12], [20], [25], [26]). Кроме того, для слабого решения $u(\cdot)$, которое получается по методу Галёркина, выполнено энергетическое неравенство

$$
\frac{1}{2} \frac{d}{d t}|u(t)|^{2}+\nu\|u(t)\|^{2} \leqslant(g, u(t)), \quad t \geqslant 0,
$$

которое нужно понимать в следующем смысле: для любой пробной функции $\psi(\cdot) \in C_{0}^{\infty}\left(\mathbb{R}_{+}\right), \psi(t) \geqslant 0$, справедливо неравенство

$$
-\frac{1}{2} \int_{0}^{+\infty}|u(s)|^{2} \psi^{\prime}(s) d s+\nu \int_{0}^{+\infty}\|u(s)\|^{2} \psi(s) d s \leqslant \int_{0}^{+\infty}(g, u(s)) \psi(s) d s .
$$

Отметим, что квадратичный оператор $B(u, u)$ обладает свойством ортогональности:

$$
(B(u, u), u)=0, \quad \forall u \in V
$$


и равенство в (5.11) формально получается после умножения уравнения (5.2) на функцию $u$ и применения соотношения

$$
\left(\partial_{t} u(t), u(t)\right)=\frac{1}{2} \frac{d}{d t}|u(t)|^{2},
$$

которое справедливо, если дополнительно известно, что $\partial_{t} u(\cdot) \in L_{2}\left(0, M ; V^{\prime}\right)$ (см. [26]). Однако для любого слабого решения уравнения (5.2), а также для построенного выше решения известно лишь, что $\partial_{t} u(\cdot) \in L_{4 / 3}\left(0, M ; V^{\prime}\right)$, поэтому использовать (5.13) напрямую нельзя. Тем не менее, для любого галёркинского приближения $u_{m}(t)$, которое является достаточно гладкой функцией класса $C^{1}$, соотношение вида (5.13) имеет место. Поэтому для него справедливо энергетическое равенство

$$
\frac{1}{2} \frac{d}{d t}\left|u_{m}(t)\right|^{2}+\nu\left\|u_{m}(t)\right\|^{2}=\left(g, u_{m}(t)\right), \quad t \geqslant 0 .
$$

Из этого равенства, с помощью предельного перехода при $m \rightarrow \infty$ в слабой топологии пространств $L_{2}(0, M ; V)$ и $L_{\infty}(0, M ; H)$, получается энергетическое неравенство (5.11) для найденного по методу Галёркина точного слабого решения $u(t)$ уравнения (5.2) (см. подробнее, например, в [12], [26]).

Таким образом, метод Галёркина позволяет построить достаточно широкое семейство слабых решений уравнения (5.2), которые удовлетворяют неравенству (5.12). Эти слабые решения формируют пространство траекторий задачи.

ОПРЕДЕЛЕНИЕ 5.1. Пространством траекторий $\mathscr{K}^{+}$3D-системы НавьеСтокса (5.2) называется совокупность всех ее слабых решений из $L_{2}^{\text {loc }}\left(\mathbb{R}_{+} ; V\right) \cap$ $L_{\infty}^{\text {loc }}\left(\mathbb{R}_{+} ; H\right)$, которые удовлетворяют неравенству $(5.12)$.

ЗАмечАниЕ 5.1. Для 3D-системы Навье-Стокса теорема единственности слабого решения задачи Коши (5.2), (5.8) остается недоказанной. Изучение этой фундаментальной проблемы признается математическим сообществом весьма актуальной. Неизвестно также, будет ли выполнено энергетическое неравенство (5.12) (и тем более равенство) для любого слабого решения системы уравнений (5.2). Однако слабые решения, которые строятся по методу Галёркина, этому неравенству удовлетворяют.

Построение траекторного аттрактора. Следуя общей схеме, изложенной в разделе 4 , введем пространства $E=H$ и $E_{0}=V^{\prime}$. Тогда систему $(5.2)$ можно записать в виде $(4.1)$, причем $F(u)=-\nu L u+B(u, u)-g(x)$. Вводятся также пространства, используемые в общей схеме:

$$
\begin{aligned}
\mathscr{F}(0, M ; E) & :=L_{2}(0, M ; V) \cap L_{\infty}(0, M ; H) \cap\left\{f(\cdot) \mid \partial_{t} f(\cdot) \in L_{4 / 3}\left(0, M ; V^{\prime}\right)\right\}, \\
\mathscr{F}\left(0, M ; E_{0}\right) & :=L_{4 / 3}\left(0, M ; V^{\prime}\right) .
\end{aligned}
$$

По этим пространствам строится пространство

$$
\mathscr{F}_{+}^{\mathrm{loc}}=L_{2}^{\mathrm{loc}}\left(\mathbb{R}_{+} ; V\right) \cap L_{\infty}^{\mathrm{loc}}\left(\mathbb{R}_{+} ; H\right) \cap\left\{v(\cdot) \mid \partial_{t} v(\cdot) \in L_{4 / 3}^{\mathrm{loc}}\left(\mathbb{R}_{+} ; V^{\prime}\right)\right\},
$$

которому, очевидно, принадлежит пространство траекторий $\mathscr{K}^{+}$системы Навье-Стокса. 
В $\mathscr{F}_{+}^{\text {loc }}$ действует полугруппа трансляций $\{T(t), t \geqslant 0\}$ :

$$
T(t) v(\cdot, s)=v(\cdot, t+s), \quad s \geqslant 0 .
$$

Заметим, что $\{T(t)\}$ отображает пространство траекторий $\mathscr{K}^{+}$в себя:

$$
T(t): \mathscr{K}^{+} \rightarrow \mathscr{K}^{+}, \quad \forall t \geqslant 0 .
$$

Действительно, если функция $u(s), s \geqslant 0$, принадлежит пространству $\mathscr{K}^{+}$, то функция $u_{h}(s):=T(h) u(s)=u(h+s), s \geqslant 0$, очевидно, является слабым решением системы (5.2) и удовлетворяет энергетическому неравенству (5.11), как и функция $u(s)$, поскольку уравнение (5.2) является автономным.

Согласно разделу 4, для построения траекторного аттрактора в пространстве траекторий $\mathscr{K}^{+}$уравнения (5.2) необходимо ввести топологию $\Theta_{+}^{\text {loc }}$ в пространстве $\mathscr{F}_{+}^{\text {loc }}$, а также определить банахово пространство $\mathscr{F}_{+}^{\mathrm{b}} \subset \mathscr{F}_{+}^{\text {loc }}$.

Топология $\Theta_{+}^{\text {loc }}$ является индуктивным пределом топологий $\Theta(0, M ; E)$ в пространствах $\mathscr{F}(0, M ; E)$, которые задаются с помощью следующей (слабой) сходимости последовательностей: по определению, последовательность функций $\left\{v_{m}(x, s)\right\}$ из $\mathscr{F}(0, M ; E)$ сходится к функции $v(x, s) \in \mathscr{F}(0, M ; E)$ в топологии $\Theta(0, M ; E)$ при $m \rightarrow \infty$, если

$$
\left\{\begin{array}{l}
v_{m}(x, s) \rightarrow v(x, s) \text { слабо в } L_{2}(0, M ; V), * \text {-слабо в } L_{\infty}(0, M ; H), \\
\partial_{t} v_{m}(x, s) \rightarrow \partial_{t} v(x, s) \text { слабо в } L_{4 / 3}\left(0, M ; V^{\prime}\right)
\end{array}\right.
$$

при $m \rightarrow \infty$.

Банахово пространство $\mathscr{F}_{+}^{\mathrm{b}} \subset \mathscr{F}_{+}^{\text {loc }}$ задается следующим образом:

$$
\mathscr{F}_{+}^{\mathrm{b}}:=\left\{v(\cdot) \in \mathscr{F}_{+}^{\mathrm{loc}} \mid\|v\|_{\mathscr{F}_{+}^{\mathrm{b}}}<\infty\right\}
$$

причем норма в $\mathscr{F}_{+}^{\mathrm{b}}$ определяется по формуле (4.3), т. е.

$$
\|v\|_{\mathscr{F}_{+}^{\mathrm{b}}}:=\sup _{t \geqslant 0}\left\{\|T(t) v\|_{L_{2}(0,1 ; V)}+\|T(t) v\|_{L(0,1 ; H)}+\left\|T(t) \partial_{t} v(\cdot)\right\|_{L_{4 / 3}^{\mathrm{b}}\left(0,1 ; V^{\prime}\right)}\right\} .
$$

Очевидно, что

$$
\mathscr{F}_{+}^{\mathrm{b}}:=L_{2}^{\mathrm{b}}\left(\mathbb{R}_{+} ; V\right) \cap L_{\infty}\left(\mathbb{R}_{+} ; H\right) \cap\left\{v(\cdot) \mid \partial_{t} v(\cdot) \in L_{4 / 3}^{\mathrm{b}}\left(\mathbb{R}_{+} ; V^{\prime}\right)\right\} .
$$

В [12] установлены два важных свойства пространства траекторий $\mathscr{K}^{+}$.

УтВеРЖДЕНИЕ 5.1. Пространство $\mathscr{K}^{+}$принадлежит $\mathscr{F}_{+}^{\mathrm{b}}$, и для любой функции и(·) $\in \mathscr{K}^{+}$справедливо неравенство

$$
\|T(t) u(\cdot)\|_{\mathscr{F}_{+}^{\mathrm{b}}} \leqslant C\|u(\cdot)\|_{L_{2}(0,1 ; H)}^{2} \mathrm{e}^{-\nu \lambda_{1} t}+R_{0}, \quad \forall t \geqslant 0
$$

где $\lambda_{1}$ - первое собственное значение оператора Cтокса L, константа $C$ зависит от $\lambda_{1}$ и $\nu$, а величина $R_{0}$ зависит от $\lambda_{1}, \nu u\|g\|_{V^{\prime}}$.

УтВеРЖДЕНИЕ 5.2. Пространство $\mathscr{K}^{+}$замкнуто в топологии $\Theta_{+}^{\mathrm{loc}}$. 
При доказательстве (5.19) используется энергетическое неравенство (5.12) и вытекающие из него стандартные априорные оценки, к которым применяется классическая лемма Гронуолла.

Из неравенства (5.19) вытекает, что множество

$$
\mathscr{P}_{0}=\left\{u \in \mathscr{K}^{+} \mid\|u\|_{\mathscr{F}_{+}^{\mathrm{b}}} \leqslant 2 R_{0}\right\}
$$

является поглощающим для полугруппы $\{T(t)\}$ на $\mathscr{K}^{+}$, причем это множество инвариантно относительно $\{T(t)\}$ :

$$
T(t) \mathscr{P}_{0} \subseteq \mathscr{P}_{0}, \quad \forall t \geqslant 0 .
$$

Легко видеть, что поглощающее множество $\mathscr{P}_{0}$ ограничено в $\mathscr{F}_{+}^{\mathrm{b}}$ и компактно в топологии $\Theta_{+}^{\text {loc }}$ (см. замечание 2.3 , которое применимо и к системе НавьеСтокса).

Проверены все условия теоремы 4.1, из которой получаем следующий основной результат.

Теорема 5.1. Трансляиионная полугруппа $\{T(t)\}$, действующая в пространстве траекторий $\mathscr{K}^{+}$системъ Навъе-Стокса (5.2), имеет траекторный аттрактор $\mathfrak{A}$ в топологии $\Theta_{+}^{\mathrm{loc}}$. Множество $\mathfrak{A}$ ограничено в $\mathscr{F}_{+}^{\mathrm{b}}$ и компактно в топологии $\Theta_{+}^{\text {loc }}$, причем $\mathfrak{A} \subset \mathscr{P}_{0}$.

Поясним свойство притяжения к траекторному аттрактору $\mathfrak{A}$.

Пусть $H^{\delta}, \delta \in \mathbb{R}$, обозначает шкалу гильбертовых пространств, отвечающую оператору $L$ в пространстве $H$, и, в частности, $H^{0}=H, H^{1}=V$ и $H^{-1}=V^{\prime}$.

Обозначим через $\Pi_{0, M}$ оператор ограничения на интервал $(0, M)$ функций, заданных на полуоси $\mathbb{R}_{+}$. Отметим, что при любом $M>0$ введенная выше топология $\Theta_{0, M}:=\Pi_{0, M} \Theta_{+}^{\text {loc }}$ сильнее, чем топология сходимости по норме в пространстве $L_{2}\left(0, M ; H^{\delta}\right)$ при $0 \leqslant \delta<1$. Это вытекает из компактности вложения

$$
L_{2}(0, M ; V) \cap\left\{v \mid \partial_{t} v \in L_{4 / 3}\left(0, M ; V^{\prime}\right)\right\} \Subset L_{2}\left(0, M ; H^{\delta}\right),
$$

которое следует из теоремы Обена (см. [20], [49], [50]).

СлеДСТВИЕ 5.1. Для любого множества $\mathfrak{B} \subset \mathscr{K}^{+}$, ограниченного в $\mathscr{F}_{+}^{\mathrm{b}}$,

$$
\operatorname{dist}_{L_{2}\left(0, M ; H^{\delta}\right)}\left(\Pi_{0, M} T(t) \mathfrak{B} ; \Pi_{0, M} \mathfrak{A}\right) \rightarrow 0 \quad(t \rightarrow+\infty), \quad \forall M>0 .
$$

Сформулируем еще один полезный результат. Пусть $\mathbf{v}=\left(v_{1}, \ldots, v_{N}\right) \in H^{N}-$ конечная система вектор-функций из $H$. Обозначим через $J_{\mathbf{v}}$ отображение из $\mathscr{F}_{+}^{\text {loc }}$ в $C\left(\mathbb{R}_{+} ; \mathbb{R}^{N}\right)$, определяемое по формуле

$$
J_{\mathbf{v}}(u)(s):=\left(\left(u(s), v_{1}\right), \ldots,\left(u(s), v_{N}\right)\right) .
$$

Как уже отмечалось, любая функция $u(s), s \geqslant 0$, из $\mathscr{F}_{+}^{\text {loc }}$ является слабо непрерывной со значениями в $H$, и поэтому образ $u(\cdot)$ под действием $J_{\mathbf{v}}$ является непрерывной по $s$ функцией со значениями в $\mathbb{R}^{N}$. Воспользуемся теперь вложением

$$
L_{\infty}(0, M ; H) \cap\left\{v \mid \partial_{t} v \in L_{4 / 3}\left(0, M ; V^{\prime}\right)\right\} \subset C_{\mathrm{w}}([0, M] ; H),
$$


где справа стоит пространство слабо непрерывных функций на $[0, M]$ со значениями в пространстве $H$ (см. [47]). Заметим, что топология $\Theta_{M}$ сильнее топологии $C_{\mathrm{w}}([0, M] ; H)$.

СлеДСТВИЕ 5.2. Для любого ограниченного в $\mathscr{F}_{+}^{\mathrm{b}}$ множества $\mathfrak{B} \subset \mathscr{K}^{+}$

$\operatorname{dist}_{C\left([0, M] ; \mathbb{R}^{N}\right)}\left(\Pi_{0, M} J_{\mathbf{v}} T(t) \mathfrak{B} ; \Pi_{0, M} J_{\mathbf{v}} \mathfrak{A}\right) \rightarrow 0 \quad(t \rightarrow+\infty), \quad \forall M \geqslant 0$.

ЗАмечАнИЕ 5.2. Вопрос о том, верно ли предельное соотношение (5.22) при $\delta=1$, остается нерешенным. То же относится к проблема притяжения траекторий к траекторному аттрактору $\mathfrak{A}$ в сильной топологии $\Theta_{+}^{\mathrm{s}, \text { loc }}$, которая определяется аналогично (5.16) с заменой слабых пределов на сильные пределы по нормам в соответствующих пространствах.

Связь с ядром 3D-системы Навье-Стокса. Рассмотрим систему (5.2) на всей оси времени $(-\infty<t<+\infty)$. Мы будем также рассматривать слабые решения $u(x, t)$ этой системы при всех $s \in \mathbb{R}$. Функция $u(\cdot) \in L_{2}^{\operatorname{loc}}(\mathbb{R} ; V) \cap$ $L_{\infty}^{\text {loc }}(\mathbb{R} ; H)$ называется полным слабым решением уравнения (5.2), если она удовлетворяет этому уравнению в смысле пространства $\mathscr{D}^{\prime}\left(\mathbb{R} ; V^{\prime}\right)$. Тогда, как известно, обобщенная производная по времени $\partial_{t} u(\cdot)$ принадлежит $L_{4 / 3}^{\text {loc }}\left(\mathbb{R} ; V^{\prime}\right)$.

Аналогично пространствам $\mathscr{F}_{+}^{\text {loc }}$ и $\mathscr{F}_{+}^{\mathrm{b}}$ определяются пространства $\mathscr{F}^{\text {loc }}$ и $\mathscr{F}^{\mathrm{b}}$. Легко видеть, что

$$
\mathscr{F}^{\mathrm{b}}:=L_{2}^{\mathrm{b}}(\mathbb{R} ; V) \cap L_{\infty}(\mathbb{R} ; H) \cap\left\{v(\cdot) \mid \partial_{t} v(\cdot) \in L_{4 / 3}^{\mathrm{b}}\left(\mathbb{R} ; V^{\prime}\right)\right\},
$$

причем норма в $\mathscr{F}^{\mathrm{b}}$ задается формулой

$$
\|v(\cdot)\|_{\mathscr{F} \mathrm{b}}:=\|v(\cdot)\|_{L_{2}^{\mathrm{b}}(\mathbb{R} ; V)}+\|v(\cdot)\|_{L_{\infty}(\mathbb{R} ; H)}+\left\|\partial_{t} v(\cdot)\right\|_{L_{4 / 3}^{\mathrm{b}}\left(\mathbb{R} ; V^{\prime}\right)} \cdot
$$

Из определения 4.2 следует, что ядро $\mathscr{K}$ системы (5.2) состоит из всех его полных слабых решений $u(x, s), s \in \mathbb{R}$, имеющих ограниченную норму в $\mathscr{F} \mathrm{b}$ и удовлетворяющих энергетическому неравенству

$$
-\frac{1}{2} \int_{-\infty}^{+\infty}|u(s)|^{2} \psi^{\prime}(s) d s+\nu \int_{-\infty}^{+\infty}\|u(s)\|^{2} \psi(s) d s \leqslant \int_{-\infty}^{+\infty}(g, u(s)) \psi(s) d s
$$

для любой пробной функции $\psi(\cdot) \in C_{0}^{\infty}(\mathbb{R}), \psi(s) \geqslant 0$.

Через $\Pi_{+}$обозначается, как обычно, оператор ограничения на полуось $\mathbb{R}_{+}$. Из теоремы 4.2 получаем следующий результат.

ТеОрема 5.2. Ядро $\mathscr{K}$ и траекторный аттрактор $\mathfrak{A}$ системъ НавъеСтокса (5.2) связаны следующим соотношением:

$$
\mathfrak{A}=\Pi_{+} \mathscr{K} .
$$

Ядро $\mathscr{K}$ ограничено в пространстве $\mathscr{F}^{\mathrm{b}}$ и компактно в топологии $\Theta^{\mathrm{loc}}$.

Имея траекторный аттрактор, построим (слабый) глобальный аттрактор 3D-системы Навье-Стокса по общей схеме, изложенной в разделе 4. 
Слабый глобальный аттрактор системы Навье-Стокса. Рассмотрим произвольную траекторию $u(t), t \geqslant 0,3 \mathrm{D}$-системы Навье-Стокса, из пространства $\mathscr{K}^{+}$. Функция $u(\cdot)$ имеет ограниченную норму в $\mathscr{F}_{+}^{\mathrm{b}}$, и, в частности, она принадлежит пространству $L_{\infty}\left(\mathbb{R}_{+} ; H\right)$, а из леммы 2.1 получаем, что $u(\cdot) \in C_{\mathrm{w}}\left(\mathbb{R}_{+} ; H\right)$. Следовательно, при каждом $t \in \mathbb{R}_{+}$значение $u(t) \in H$ корректно задано, и в силу (2.5) имеет место неравенство

$$
|u(t)| \leqslant\|u(\cdot)\|_{L_{\infty}\left(\mathbb{R}_{+} ; H\right)}, \quad \forall t \geqslant 0 .
$$

Рассмотрим сечение любого ограниченного в $\mathscr{F}_{+}^{\mathrm{b}}$ множества $\mathfrak{B} \subset \mathscr{K}^{+}$траекторий системы (5.2) в момент времени $t \geqslant 0$ :

$$
\mathfrak{B}(t)=\{u(t) \mid u \in \mathfrak{B}\} \subset H .
$$

Пусть $\mathfrak{A}$ - траекторный аттрактор системы Навье-Стокса. Рассмотрим его сечение в момент времени $t=0$ :

$$
\mathfrak{A}(0):=\{u(0) \mid u \in \mathfrak{A}\} \subset H .
$$

Из строгой инвариантности множества $\mathfrak{A}$ относительно трансляционной полугруппы следует, что

$$
\mathfrak{A}(0)=\mathfrak{A}(t), \quad \forall t \geqslant 0 .
$$

Ясно, что множество $\mathfrak{A}(0)$ ограничено по норме $H$. Кроме того, из свойств траекторного аттрактора следует, что $\mathfrak{A}(0)$ замкнуто в сильной топологии $H$. Действительно, множество $\mathfrak{A}$ компактно в топологии $\Theta_{+}^{\text {loc }}$, которая сильнее топологии $C_{\mathrm{w}}^{\mathrm{loc}}\left(\mathbb{R}_{+} ; H\right)$ (см. следствие 5.2$)$, поэтому $\mathfrak{A}$ компактно в $C_{\mathrm{w}}^{\text {loc }}\left(\mathbb{R}_{+} ; H\right)$, и, следовательно, его сечение $\mathfrak{A}(0)$ слабо компактно в $H$, а значит, слабо замкнуто в $H$. Как известно, любое слабо замкнутое подмножество гильбертова пространства является сильно замкнутым (см. [27]). Значит, $\mathfrak{A}(0)$ сильно замкнуто в пространстве $H$.

УТВеРЖДЕНИЕ 5.3. Множество $\mathscr{A}:=\mathfrak{A}(0)$ является слабым глобалъным аттрактором системы (5.2). Точнее, $\mathscr{A}$ ограничено и замкнуто в $H$, , кроме mого,

1) для любого множества $\mathfrak{B} \subset \mathscr{K}^{+}$, ограниченного по норме $\mathscr{F}_{+}^{\mathrm{b}}$, его сечение $\mathfrak{B}(t)$ притягивается $\kappa \mathscr{A}$ в слабой топологии $H$ при $t \rightarrow+\infty$,

2) $\mathscr{A}$ является минимальным ограниченным и замкнутым множеством в пространстве $H$, которое удовлетворяет 1$)$.

ДокАЗАтЕЛьство. Ограниченность и замкнутость множества $\mathscr{A}$ уже установлены.

Чтобы проверить 1), достаточно воспользоваться следствием 5.2 и положить $M=0$ в формуле (5.23).

Проверим свойство 2). Пусто $\widetilde{\mathscr{A}}$ - произвольное ограниченное и замкнутое подмножество из $H$, которое слабо притягивает сечения любых ограниченных множеств $\mathfrak{B} \subset \mathscr{K}^{+}$, т. е. для любой слабой окрестности $\mathscr{O}_{\mathrm{w}}(\widetilde{\mathscr{A}})$

$$
\mathfrak{B}(t) \subset \mathscr{O}_{\text {ш }}(\tilde{\mathscr{A}}) \quad \text { при всех } t \geqslant \tau
$$


где $\tau=\tau\left(\mathscr{O}_{\mathrm{w}}\right)$. В частности, (5.26) должно выполняться и для множества траекторий $\mathfrak{B}=\mathfrak{A}$, так как, множество $\mathfrak{A}$ ограничено в $\mathscr{F}_{+}^{\mathrm{b}}$, т. е.

$$
\mathfrak{A}(t) \subset \mathscr{O}_{\mathrm{w}}(\tilde{\mathscr{A}}) \quad \text { при всех } t \geqslant \tau,
$$

Но, в силу строгой инвариантности траекторного аттрактора, $\mathfrak{A}(t)=\mathfrak{A}(0)$ при всех $t \geqslant 0$. Поэтому из $(5.27)$ находим, что

$$
\mathscr{A}=\mathfrak{A}(0) \subset \mathscr{O}_{\mathrm{w}}(\tilde{\mathscr{A}})
$$

для любой слабой окрестности $\mathscr{O}_{\mathrm{w}}(\widetilde{\mathscr{A}})$ множества $\widetilde{\mathscr{A}}$ в $H$. Тогда, в силу замкнутости и слабой компактности $\widetilde{\mathscr{A}}$ в пространстве $H$, множество $\mathscr{A}$ принадлежит $\widetilde{\mathscr{A}}$. Следовательно, $\mathscr{A}$ содержится в любом ограниченном и замкнутом множестве, которое обладает свойством слабого притяжения, т. е. $\mathscr{A}$ - минимально в смысле 2).

В заключение сформулируем гипотезу, которую, быть может, удастся доказать без использования теоремы единственности задачи Коши для 3D-системы Навье-Стокса, остающейся актуальной нерешенной проблемой (см. также [66]).

ГиПотезА 5.1. Множество $\mathscr{A}=\mathfrak{A}(0)$ компактно в $H$ и притягивает сечения любых ограниченных множеств $\mathfrak{B} \subset \mathscr{K}^{+}$в сильной топологии $H$, т.е. множество $\mathfrak{A}(0)$ является сильным глобальным аттрактором. Последнее означает, что выполнено соотношение

$$
\operatorname{dist}_{H}(\mathfrak{B}(t), \mathscr{A}) \rightarrow 0 \quad(t \rightarrow+\infty)
$$

\section{6. Траекторный аттрактор нелинейного волнового уравнения}

Слабые решения волнового уравнения. Рассматривается следующее диссипативное гиперболическое уравнение:

$$
\partial_{t}^{2} u+\gamma \partial_{t} u=\Delta u-f(u)+g(x),\left.\quad u\right|_{\partial \Omega}=0, \quad t \geqslant 0 .
$$

Здесь $x \in \Omega \Subset \mathbb{R}^{n}, \gamma>0$ - коэффициент диссипации, $g(x) \in L_{2}(\Omega)$ - внешняя сила. Нелинейная функция $f(v)$ принадлежит $C(\mathbb{R})$ и удовлетворяет следующим условиям:

$$
\begin{gathered}
|f(v)| \leqslant \gamma_{0}\left(|v|^{p-1}+1\right), \quad p>1, \quad \gamma_{0}>0, \\
\gamma_{1}|v|^{p}-C_{1} \leqslant F(v), \quad F(v):=\int_{0}^{v} f(w) d w, \\
\gamma_{2} F(v)-C_{2} \leqslant f(v) v, \quad \forall v \in \mathbb{R} \quad\left(\gamma_{i}, C_{i}>0, \quad i=1,2\right) .
\end{gathered}
$$

ЗАмЕчАниЕ 6.1. Волновое уравнение (6.1) рассматривалось в разделе 2 при дополнительных предположениях, что $f(\cdot) \in C^{1}(\mathbb{R})$ и $p=\rho+2 \leqslant 2(n-1) /(n-2)$ при $n \geqslant 3$, а также выполнено неравенство (I.2.30). Тогда, как известно, соответствующая задача Коши однозначно разрешима и порождаемая ею полугруппа имеет (обычный) глобальный аттрактор. 
В этом разделе будет построен траекторный аттрактор для уравнения (6.1) без ограничений на степень $p$ полиномиального роста функции $f(u)$.

Пусть задана функция $u(x, t)$, принадлежащая $L_{\infty}\left(0, M ; L_{p}(\Omega)\right)$. Из $(6.2)$ находим, что

$$
f(u(\cdot)) \in L_{\infty}\left(0, M ; L_{q}(\Omega)\right),
$$

где $1 / p+1 / q=1$. Кроме того, имеет место неравенство

$$
\|f(u(\cdot))\|_{L_{\infty}\left(0, M ; L_{q}(\Omega)\right)}^{q} \leqslant \gamma_{0}^{\prime}\left(\|u(\cdot)\|_{L_{\infty}\left(0, M ; L_{p}(\Omega)\right)}^{p}+1\right) .
$$

Если сверх того известно, что $u(x, t) \in L_{\infty}\left(0, M ; H_{0}^{1}(\Omega)\right)$, то функция $\Delta u(x, t)+$ $g(x)$ принадлежит $L_{\infty}\left(0, M ; H^{-1}(\Omega)\right)$. Тогда при $p \leqslant 2$, в силу $(6.5)$, правая часть уравнения (6.1) принадлежит пространству $L_{\infty}\left(0, M ; H^{-1}(\Omega)\right)$. Рассмотрим случай $p>2$. С помощью перехода к двойственным соотношениям в теореме вложения Соболева получаем, что $L_{q}(\Omega) \subset H^{-r}(\Omega)$ при $r \geqslant n(1 / 2-1 / p)$. Если $r \geqslant 1$, то правая часть уравнения (6.1) принадлежит $L_{\infty}^{\text {loc }}\left(0, M ; H^{-r}(\Omega)\right)$, где $r:=\max \{1, n(1 / 2-1 / p)\}$. Следовательно, если $u(\cdot) \in L_{\infty}^{\operatorname{loc}}\left(0, M ; L_{p}(\Omega) \cap H_{0}^{1}(\Omega)\right)$, то уравнение (6.1) можно понимать в смысле пространства обобщенных функций $D^{\prime}\left(0, M ; H^{-r}(\Omega)\right)$.

Функция $u(x, t), x \in \Omega, t \in(0, M)$, называется слабым решением уравнения (6.1), если

$$
u(\cdot) \in L_{\infty}\left(0, M ; L_{p}(\Omega) \cap H_{0}^{1}(\Omega)\right), \quad \partial_{t} u(x, t) \in L_{\infty}\left(0, M ; L_{2}(\Omega)\right),
$$

и $u(x, t)$ удовлетворяет $(6.1)$ в пространстве $D^{\prime}\left(0, M ; H^{-r}(\Omega)\right)$ (см. [20]). При этом из уравнения (6.1) следует, что $\partial_{t}^{2} u \in L_{\infty}^{\operatorname{loc}}\left(0, M ; L_{q}(\Omega)+H^{-1}(\Omega)\right)$.

Будут рассматриваться глобальные слабые решения $u(x, t), x \in \Omega, t \geqslant 0$, для которых выполнено (6.6) при каждом $M>0$, и уравнение (6.1) изучается в пространстве $D^{\prime}\left(\mathbb{R}_{+} ; H^{-r}(\Omega)\right)$.

Отметим, что если $u(x, t)$ - глобальное слабое решение уравнения $(6.1)$, то $u(\cdot) \in C\left(\mathbb{R}_{+} ; L_{2}(\Omega)\right)$, а $\partial_{t} u(\cdot) \in C\left(\mathbb{R}_{+} ; H^{-r}(\Omega)\right)$. Имеет место более сильный результат.

УтВеРЖДЕНИЕ 6.1. Пусть $u(x, t)$ - слабое решение (6.1). Тогда

$$
u(\cdot) \in C_{\mathrm{w}}\left(\mathbb{R}_{+} ; H_{0}^{1}(\Omega) \cap L_{p}(\Omega)\right), \quad \partial_{t} u(\cdot) \in C_{\mathrm{w}}\left(\mathbb{R}_{+} ; L_{2}(\Omega)\right),
$$

где, напомним, $C_{\mathrm{w}}\left(\mathbb{R}_{+} ; E\right)$ обозначает пространство слабо непрерывных функиий в соответствующем пространстве $E=H_{0}^{1}(\Omega) \cap L_{p}(\Omega)$ или $E=L_{2}(\Omega)$. Кроме того, при любом $\alpha \in \mathbb{R}$ функиия

$$
\xi_{u}(t):=\|u(\cdot, t)\|^{2}+\left|\partial_{t} u(\cdot, t)+\alpha u(\cdot, t)\right|^{2}+\|u(\cdot, t)\|_{L_{p}(\Omega)}^{p}
$$

является полунепрерывной снизу по $t \geqslant 0$. Здесь и далее $\|\cdot\| u|\cdot|$ обозначают обычные нормы в пространствах $H_{0}^{1}(\Omega)$ и $L_{2}(\Omega)$ соответственно.

Доказательство, которое приведено в [12], использует лемму Лионса-Мадженеса 2.1. 
Пусть $v(x) \in H_{0}^{1}(\Omega) \cap L_{p}(\Omega)$ и $v_{1}(x) \in L_{2}(\Omega)$. Рассмотрим функционал

$$
J_{\alpha}\left(v, v_{1}\right)=\int_{\Omega}\left(|\nabla v(x)|^{2}+\left|v_{1}(x)+\alpha v(x)\right|^{2}+2 F(v(x))\right) d x,
$$

зависящий от вещественного параметра $\alpha$. Из (6.2) и (6.3) следует, что

$$
\begin{aligned}
\|v\|^{2}+\left|v_{1}+\alpha v\right|^{2}+2 \gamma_{1}\|v\|_{L_{p}(\Omega)}^{p}-C_{1}^{\prime} \leqslant J_{\alpha}\left(v, v_{1}\right) & \\
& \leqslant\|v\|^{2}+\left|v_{1}+\alpha v\right|^{2}+2 \gamma_{0}^{\prime \prime}\|v\|_{L_{p}(\Omega)}^{p}+C_{0}^{\prime} .
\end{aligned}
$$

СлЕДСтвиЕ 6.1. Если $и(\cdot, t)$ - слабое решение (6.1), то вещественная функиия

$$
z(t):=J_{\alpha}\left(u(\cdot, t), \partial_{t} u(\cdot, t)\right), \quad t \geqslant 0,
$$

является полунепрерьвной снизу на $\mathbb{R}_{+}$.

Отметим, что при $\alpha=0$ функционал $J_{0}\left(v, \partial_{t} v\right)$ совпадает с обычным функционалом энергии для уравнения (6.1).

Пусть $u(\cdot, t)$ - слабое решение $(6.1)$, полученное с помощью метода Галёркина. Предположим, что $0<\alpha<\gamma$ и

$$
0<\alpha(\gamma-\alpha)<\lambda_{1}
$$

где $\lambda_{1}-$ первое собственное значение оператора Лапласа:

$$
-\Delta u_{1}=\lambda_{1} u_{1},\left.\quad u_{1}\right|_{\partial \Omega}=0 .
$$

Тогда существуют такие положительные числа $\delta_{\alpha}>0$ и $\rho_{\alpha}>0$, что функция $z(t)=J_{\alpha}\left(u(\cdot, t), \partial_{t} u(\cdot, t)\right)$ удовлетворяет следующему дифференциальному неравенству:

$$
\frac{d}{d t} z(t)+\delta_{\alpha} z(t) \leqslant \frac{2}{\gamma-\alpha}|g|^{2}+\rho_{\alpha}
$$

причем для $\delta_{\alpha}$ и $\rho_{\alpha}$ имеются следующие явные выражения:

$$
\begin{aligned}
& \delta_{\alpha}=\min \left\{\gamma-\alpha, 2 \alpha\left(1-\frac{(\gamma-\alpha) \alpha}{\lambda_{1}}\right), \alpha \gamma_{2}\right\}, \quad \delta_{\alpha}>0, \\
& \rho_{\alpha}=2 \alpha C_{2} \mu(\Omega)+\left(\alpha \gamma_{2}-\delta_{\alpha}\right) 2 C_{1} \mu(\Omega) .
\end{aligned}
$$

Неравенство (6.13) доказано в [12] для достаточно гладких решений уравнения (6.1), а также для любых галёркинских приближений этого уравнения. Однако для любого слабого решения волнового уравнения (6.1) это неравенство не установлено. Это обстоятельство существенно влияет на выбор пространства траекторий волнового уравнения (6.1), а также на конструкцию траекторного аттрактора, которая описана ниже.

Из неравенства (6.13) в [12] выведен следующий результат.

УТВЕРЖДЕНИЕ 6.2. Если функиия $z(t)$ удовлетворяет (6.13), то

$$
z(t) \leqslant R_{\alpha}+z(0) \mathrm{e}^{-\delta_{\alpha} t}, \quad R_{\alpha}=\rho_{\alpha} \delta_{\alpha}^{-1}+2(\gamma-\alpha)^{-1} \delta_{\alpha}^{-1}|g|^{2} .
$$


Пространство траекторий для волнового уравнения. Зафиксируем теперь число $N>0$ и определим пространство траекторий $\mathscr{K}^{+}(N)$ уравнения (6.1).

ОПРЕДЕЛЕНИЕ 6.1. Пространство $\mathscr{K}^{+}(N)$ является объединением глобальных слабых решений $u(\cdot, t), t \geqslant 0$, уравнения $(6.1)$, для которых справедливо неравенство

$$
z(t):=J_{\alpha}\left(u(t), \partial_{t} u(t)\right) \leqslant R_{\alpha}+N \exp \left(-\delta_{\alpha} t\right), \quad \forall t \geqslant 0,
$$

где $\alpha \in(0, \gamma)$ - любое число, удовлетворяющее (6.12). Здесь $R_{\alpha}$ взято из (6.16), а $\delta_{\alpha}$ определено в (6.14).

УтВеРЖДЕНИЕ 6.3. Пустъ $u_{0}(x) \in H_{0}^{1}(\Omega) \cap L_{p}(\Omega), u_{1}(x) \in L_{2}(\Omega)$, причем $z_{0}=J_{\alpha}\left(u_{0}, u_{1}\right) \leqslant N$ для всех $\alpha \in(0, \gamma)$, которые удовлетворяют (6.12). Тогда существует по крайней мере одна траектория $u(\cdot) \in \mathscr{K}^{+}(N)$ такая, что

$$
\left.u\right|_{t=0}=u_{0}(x),\left.\quad \partial_{t} u\right|_{t=0}=u_{1}(x) .
$$

Слабое решение (траекторию) $u(\cdot) \in \mathscr{K}^{+}(N)$ можно построить, например, как предел галёркинских приближений $u_{m}(x, t)=\sum_{j=1}^{m} a_{j, m}(t) w_{j}(x)$ при $m \rightarrow \infty$. Здесь $\left\{w_{j}(x)\right\}_{j \in \mathbb{N}}$ - полная система функций в пространстве $H_{0}^{1}(\Omega) \cap L_{p}(\Omega)$. Функция $u_{m}(\cdot, t)$ удовлетворяет системе ОДУ

$$
\partial_{t}^{2} u_{m}+\gamma \partial_{t} u_{m}=P_{m} \Delta u_{m}-P_{m} f\left(u_{m}\right)+P_{m} g(x)
$$

и начальным условиям

$$
\left.u_{m}\right|_{t=0}=u_{0 m}(x),\left.\quad \partial_{t} u_{m}\right|_{t=0}=u_{1 m}(x),
$$

причем $u_{0 m}(x) \rightarrow u_{0}(x)$ сильно в $H_{0}^{1}(\Omega) \cap L_{p}(\Omega)$ и $u_{1 m}(x) \rightarrow u_{1}(x)$ сильно в $L_{2}(\Omega)$ при $m \rightarrow \infty$. В уравнении (6.19) $P_{m}-$ ортогональный проектор из $L_{2}(\Omega)$ на линейную оболочку функций $\left\{w_{1}(x), \ldots, w_{m}(x)\right\}$. Легко устанавливается, что

$$
z_{m}(0)=J_{\alpha}\left(u_{0 m}, u_{1 m}\right) \rightarrow J_{\alpha}\left(u_{0}, u_{1}\right)=z_{0} \quad(m \rightarrow \infty) .
$$

Обозначим $z_{m}(t)=J_{\alpha}\left(u_{m}(\cdot, t), \partial_{t} u_{m}(\cdot, t)\right), t \geqslant 0$. Как уже отмечалось, любое галёркинское приближение $u_{m}(t)$ удовлетворяет дифференциальному неравенству (6.13). Тогда в силу утверждения 6.2 функция $z_{m}(t)$ удовлетворяет неравенству

$$
z_{m}(t) \leqslant R_{\alpha}+z_{m}(0) \mathrm{e}^{-\delta_{\alpha} t}, \quad \forall t \geqslant 0
$$

при любом $\alpha$, удовлетворяющем (6.12). Применяя оценки (6.5), (6.10), а также неравенство (6.21), заключаем, что последовательность функций $\left\{u_{m}(t)\right\}$ является ограниченной в пространстве $L_{\infty}\left(\mathbb{R}_{+} ; L_{p}(\Omega) \cap H_{0}^{1}(\Omega)\right),\left\{\partial_{t} u_{m}(s)\right\}$ ограничена в $L_{\infty}\left(\mathbb{R}_{+} ; L_{2}(\Omega)\right)$ и $\left\{\partial_{t}^{2} u_{m}(t)\right\}$ ограничена в $L_{\infty}\left(\mathbb{R}_{+} ; H^{-r}(\Omega)\right)$.

Переходя к пределу для последовательности галёркинских приближений $\left\{u_{m}(t)\right\}$ при $m \rightarrow \infty$, находим функцию $u(t) \in L_{\infty}\left(\mathbb{R}_{+} ; L_{p}(\Omega) \cap H_{0}^{1}(\Omega)\right), \partial_{t} u(t) \in$ $L_{\infty}\left(\mathbb{R}_{+} ; L_{2}(\Omega)\right)$, причем $\partial_{t}^{2} u(t) \in L_{\infty}\left(\mathbb{R}_{+} ; H^{-r}(\Omega)\right)$. Кроме того, $u(\cdot)$ является 
слабым решением (6.1), удовлетворяющим начальным условиям (6.18). Можно проверить, что $u(\cdot)$ удовлетворяет неравенству (6.17) (подробнее см. [12]).

Как обычно, на пространстве траекторий $\mathscr{K}^{+}(N)=\{u(s), s \geqslant 0\}$ рассматривается полугруппа трансляций $\{T(t)\}$.

Введем пространства $\mathscr{F}_{+}^{\text {loc } и ~ \mathscr{F}} \mathscr{F}_{+}^{\mathrm{b}}$ для уравнения (6.1). Обозначим $E=L_{2}(\Omega)$. По определению,

$$
\begin{gathered}
\mathscr{F}(0, M ; E):=L_{\infty}\left(0, M ; L_{p}(\Omega) \cap H_{0}^{1}(\Omega)\right) \cap\left\{\partial_{t} v \in L_{\infty}\left(0, M ; L_{2}(\Omega)\right)\right\} \\
\cap\left\{\partial_{t}^{2} v \in L_{\infty}\left(0, M ; H^{-r}(\Omega)\right)\right\} .
\end{gathered}
$$

Пространство $\mathscr{F}(0, M ; E)$ порождает $\mathscr{F}_{+}^{\text {loc }}$ и $\mathscr{F}_{+}^{\mathrm{b}}$ (см. раздел 4$)$. Легко видеть, что

$\mathscr{F}_{+}^{\mathrm{b}}=L_{\infty}\left(\mathbb{R}_{+} ; L_{p}(\Omega) \cap H_{0}^{1}(\Omega)\right) \cap\left\{\partial_{t} v \in L_{\infty}\left(\mathbb{R}_{+} ; L_{2}(\Omega)\right)\right\} \cap\left\{\partial_{t}^{2} v \in L_{\infty}\left(\mathbb{R}_{+} ; H^{-r}\right)\right\}$.

Топология $\Theta(0, M ; E)$ в пространстве $\mathscr{F}(0, M ; E)$ совпадает со следующей топологией сходимости: последовательность $\left\{v_{m}(\cdot)\right\}$ сходится к $v(\cdot)$ при $m \rightarrow \infty$ в $\Theta(0, M ; E)$, если

$$
\begin{array}{ccl}
v_{m}(s) \rightarrow v(s) & (m \rightarrow \infty) & \text { *-слабо в } L_{\infty}\left(0, M ; H_{0}^{1}(\Omega) \cap L_{p}(\Omega)\right), \\
\partial_{t} v_{m}(s) \rightarrow \partial_{t} v(s) & (m \rightarrow \infty) & \text { *-слабо в } L_{\infty}\left(0, M ; L_{2}(\Omega)\right), \\
\partial_{t}^{2} v_{m}(s) \rightarrow \partial_{t}^{2} v(s) & (m \rightarrow \infty) & \text { *-слабо в } L_{\infty}\left(0, M ; H^{-r}(\Omega)\right) .
\end{array}
$$

Топология $\Theta(0, M ; E)$ порождает топологию индуктивного предела $\Theta_{+}^{\text {loc }}$ в пространстве $\mathscr{F}_{+}^{\text {loc }}$.

УТВЕРЖДЕНИЕ 6.4. Для любого $N \geqslant 0$ пространство траекторий $\mathscr{K}^{+}(N)$ ограничено в $\mathscr{F}_{+}^{\mathrm{b}}$.

Доказательство непосредственно вытекает из (6.17) и (6.10) при фиксированном $\alpha$. В самом деле, если $u(s) \in \mathscr{K}^{+}(N)$, то

$$
\|u(t)\|^{2}+\left|\partial_{t} u(t)+\alpha u(t)\right|^{2}+2 \gamma_{1}\|u(t)\|_{L_{p}(\Omega)}^{p}-C_{1}^{\prime} \leqslant z(t) \leqslant R_{\alpha}+N, \quad \forall t \geqslant 0 .
$$

Из уравнения (6.1) и оценки (6.5) следует, что $\partial_{t}^{2} u(s)$ ограничена в пространстве $L_{\infty}\left(\mathbb{R}_{+} ; H^{-r}(\Omega)\right)$. В [12] также доказано следующее утверждение.

УТвЕРЖДЕНИЕ 6.5. Пространство $\mathscr{K}^{+}(N)$ замкнуто в топологии $\Theta_{+}^{\text {loc }}$.

Построение траекторного аттрактора. Зафиксируем произвольное число $\alpha=\alpha_{0}$, удовлетворяющее (6.12). Рассмотрим множество

$$
\mathscr{P}_{0}=\left\{u(\cdot) \in \mathscr{F}_{+}^{\mathrm{b}} \mid z(t) \leqslant 2 R_{\alpha_{0}}, \forall t \geqslant 0\right\},
$$

где $z(t)$ составлено из $u(t)$ по формуле (6.11). Из (6.10) и (6.5) получаем, что множество $\mathscr{P}_{0}$ ограничено в $\mathscr{F}_{+}^{\mathrm{b}}$ и компактно в топологии $\Theta_{+}^{\text {loc }}$. Из неравенства (6.17) следует, что $\mathscr{P}_{0}$ является поглощающим множеством для $\mathscr{K}^{+}(N)$ при любом $N>0$.

Строим траекторный аттрактор для уравнения (6.1), следуя схеме раздела 4. 
Обозначим через $\mathscr{K}$ ядро уравнения (6.1), которое состоит из всех его слабых решений $u(t), t \in \mathbb{R}$, которые ограничены в пространстве

$$
\mathscr{F}^{\mathrm{b}}=L_{\infty}\left(\mathbb{R} ; L_{p}(\Omega) \cap H_{0}^{1}(\Omega)\right) \cap\left\{\partial_{t} v \in L_{\infty}\left(\mathbb{R} ; L_{2}(\Omega)\right)\right\} \cap\left\{\partial_{t}^{2} v \in L_{\infty}\left(\mathbb{R} ; H^{-r}(\Omega)\right)\right\}
$$

и удовлетворяют неравенству

$$
z(t)=J_{\alpha}\left(u(t), \partial_{t} u(t)\right) \leqslant R_{\alpha}, \quad \forall t \in \mathbb{R}
$$

при всех $\alpha$, которые удовлетворяют (6.12).

Применяя общие теоремы 4.1 и 4.2 , из утверждений 6.4 и 6.5 получаем основной результат.

Теорема 6.1. Пусть $g(x) \in L_{2}(\Omega)$ u $f(v)$ удовлетворяет (6.2)-(6.4). Тогда при любом $N>0$ полугруппа трансляиий $\{T(t)\}$ на $\mathscr{K}^{+}(N)$ имеет траекторный аттрактор $\mathfrak{A} \subseteq \mathscr{P}_{0}$. Множество $\mathfrak{A}$ ограничено в $\mathscr{F}_{+}^{\mathrm{b}}$ и компактно в топологии $\Theta_{+}^{\text {loc }}$. Кроме того, $\mathfrak{A}$ не зависит от $N$ и имеет место равенство

$$
\mathfrak{A}=\Pi_{+} \mathscr{K},
$$

где $\mathscr{K}$ - ядро уравнения (6.1). Множество $\mathscr{K}$ ограничено в $\mathscr{F}^{\mathrm{b}}$ и компактно в топологии $\Theta^{\mathrm{loc}}$.

Отметим, что топология $\Theta_{+}^{\text {loc }}$ сильнее топологии сходимости в пространстве $C^{\text {loc }}\left(\mathbb{R}_{+} ; H_{0}^{1-\delta}(\Omega)\right) \cap C^{1, \text { loc }}\left(\mathbb{R}_{+} ; H^{-\delta}(\Omega)\right)$ при любом $\left.\left.\delta \in\right] 0,1\right]$.

СЛЕДСТВИЕ 6.2. Для любого множества $\mathfrak{B} \subset \mathscr{K}^{+}(N)$

$$
\operatorname{dist}_{C\left([0, M] ; H_{0}^{1-\delta}(\Omega)\right) \cap C^{1}\left([0, M] ; H^{-\delta}(\Omega)\right)}\left(\Pi_{0, M} T(t) \mathfrak{B}, \Pi_{0, M} \mathscr{K}\right) \rightarrow 0 \quad(t \rightarrow+\infty)
$$

при каждом $M>0$.

ЗАмЕчАниЕ 6.2. Мы построили траекторный аттрактор диссипативного гиперболического уравнения (6.1) в слабой топологии $\Theta_{+}^{\mathrm{w}, \text { loc }}$ пространства $\mathscr{F}_{+}^{\text {loc }}$, в котором также имеется сильная топология $\Theta_{+}^{\mathrm{s}, \mathrm{loc}}$. Однако доказать притяжение к аттрактору в сильной топологии не удается. Также остается открытым вопрос, можно ли в соотношении (6.24) положить $\delta=1$.

Слабый глобальный аттрактор. Построив траекторный аттрактор $\mathfrak{A}$ для уравнения (6.1), найдем для него слабый глобальный аттрактор в фазовом пространстве

$$
\mathscr{E}:=\left[H_{0}^{1}(\Omega) \cap L_{p}(\Omega)\right] \times L_{2}(\Omega),
$$

воспользовавшись общей конструкцией из раздела 4.

Пусть $u(s), s \geqslant 0$, - любая траектория уравнения (6.1) из пространства $\mathscr{K}^{+}(N)$. Удобно исследовать пару функций $y(s):=\left(u(s), \partial_{t} u(s)\right), s \geqslant 0$. Из утверждений 6.1 и 6.4 следует, что $y(\cdot) \in C_{\mathrm{w}}\left(\mathbb{R}_{+} ; \mathscr{E}\right)$ и $y(\cdot) \in L_{\infty}\left(\mathbb{R}_{+} ; \mathscr{E}\right)$. Для любого ограниченного (в $\left.\mathscr{F}_{+}^{\mathrm{b}}\right)$ множества $\mathfrak{B}$ траекторий из $\mathscr{K}^{+}(N)$ рассмотрим его сечение в момент времени $t$ :

$$
\mathfrak{B}(t):=\left\{y(t)=\left(u(t), \partial_{t} u(t)\right) \mid u \in \mathfrak{B}\right\} \subset \mathscr{E}, \quad t \geqslant 0 .
$$


Пусть $\mathfrak{A}$ - траекторный аттрактор уравнения (6.1). Рассмотрим сечение $\mathfrak{A}$ в момент времени $t$ :

$$
\mathfrak{A}(t):=\left\{\left(u(t), \partial_{t} u(t)\right) \mid u \in \mathfrak{A}\right\} \subset \mathscr{E} .
$$

Применяем теорему 4.3 и получаем следующий результат.

УтВеРЖДЕНИЕ 6.6. Множество $\mathscr{A}:=\mathfrak{A}(0) \subset \mathscr{E}$ является слабим глобальным аттрактором уравнения (6.1), т.е. множество $\mathscr{A}$ ограничено и замкнуто в $\mathscr{E}$, а кроме того,

1) $\mathscr{A}$ притягивает сечения $\mathfrak{B}(t)$ любого ограниченного (в $\left.\mathscr{F}_{+}^{\mathrm{b}}\right)$ множества $\mathfrak{B} \subset \mathscr{K}^{+}(N)$ при $t \rightarrow \infty$ в слабой топологии $\mathscr{E} ;$

2) $\mathscr{A}$ - минимальное замкнутое множество, удовлетворяющее 1).

ЗАмЕчАниЕ 6.3. Если нелинейная функция $f(v)$ в уравнении (6.1) удовлетворяет условиям, которые обеспечивают однозначную разрешимость соответствующей задачи Коши (см., например, раздел 2 из главы I), то множество $\mathscr{A}$, построенное в утверждении 6.6, совпадает с обычным (сильным) глобальным аттрактором полугруппы $\{S(t)\}$ уравнения (6.1) в фазовом пространстве $E=$ $H_{0}^{1}(\Omega) \times L_{2}(\Omega)$.

\section{7. Аттракторы эллиптических уравнений}

В этом разделе мы обсудим приложение теории траекторных аттракторов к исследованию некоторой модельной эллиптической задачи в неограниченном цилиндре $\Omega:=\mathbb{R} \times \omega$, где $\omega$ - ограниченная область в $\mathbb{R}^{n}$ с гладкой границей. Рассматривается уравнение

$$
\mathbf{a}\left(\partial_{t}^{2} u+\Delta_{x} u\right)-\gamma \partial_{t} u-f(u)=g,\left.\quad u\right|_{\partial \omega}=0,
$$

которое является эллиптическим по совокупности аргументов $(t, x) \in \Omega \subset \mathbb{R}^{n+1}$. Векторная функция $u=u(x, t)=\left(u^{1}, \ldots, u^{k}\right)$ является неизвестной, оператор Лапласа $\Delta_{x}$ действует по переменной $x \in \omega$. В уравнении (7.1) а и $\gamma$ - заданные $(k \times k)$-матрицы, причем матрица диффузии а является положительно определенной и симметрической. Предполагается, что нелинейная вектор-функция $f(u)$ и внешняя сила $g(x)$ удовлетворяют ряду условий, которые будут сформулированы ниже.

Эллиптические уравнения вида (7.1) возникают, например, при изучении положений равновесия для соответствующей системы уравнений реакции-диффузии в неограниченной цилиндрической области $\Omega$ :

$$
\partial_{\eta} u=\mathbf{a}\left(\partial_{t}^{2} u+\Delta_{x} u\right)-\gamma \partial_{t} u-f(u)-g .
$$

Здесь скалярная переменная $\eta$ играет роль физического времени, a $t$ является пространственной переменной. В этом случае дополнительный член $\gamma \partial_{t} u$ описывает снос реагирующих химических веществ вдоль оси цилиндра.

Другим естественным источником уравнений вида (7.1) является задача о нахождении специальных решений вида бегущей волны для системы реакциидиффузии:

$$
\partial_{\eta} v=\mathbf{a}\left(\partial_{t}^{2} v+\Delta_{x} v\right)-f(v)-g
$$


Действительно, подстановка $v(\eta,(t, x)):=u(t+\gamma \eta, x)$ в последнее уравнение приводит к задаче (7.1). В этом случае коэффициент $\gamma$ играет роль скорости бегущей волны (которая также может быть неизвестной в физических приложениях и должна находиться из условия устойчивости, см. [67], [68]).

Следует отметить, что, в отличие от примеров, рассмотренных в предыдущих разделах, задача (7.1) не является эволюционной. Поэтому применение методов теории динамических систем к ее исследованию может показаться искусственным, а более логичным выглядит использование качественных методов теории эллиптических уравнений (к примеру, вариационных методов). Действительно, в относительно простых случаях (например, для скалярного уравнения или для системы уравнений, допускающей инвариантный конус) исчерпывающее описание решений может быть получено без привлечения динамики (см. [69], [68] и цитированную там литературу). Однако для более сложных уравнений и систем пространственная структура положений равновесия в неограниченных областях может быть весьма нерегулярной и даже порождать так называемый пространственный хаос. В этом случае стандартные методы эллиптической теории малоэффективны, а более целесообразным становится подход, связанный с привлечением идей и методов теории динамических систем.

Динамический метод исследования эллиптических уравнений (так называемый метод пространственной динамической системы) был впервые использован, по-видимому, в работах К. Киршгесснера (см. [70]), где строились пространственные центральные многообразия для уравнений вида (7.1) вблизи точки бифуркации. В дальнейшем динамический подход развивался и весьма успешно использовался многими авторами (см. [71]-[79]). В данной статье мы ограничимся обсуждением результатов, связанных только с использованием теории аттракторов.

Основная идея этого метода состоит в рассмотрении вспомогательной задачи на полуцилиндре $\Omega_{+}:=\mathbb{R}_{+} \times \omega$

$$
\left\{\begin{array}{l}
\mathbf{a}\left(\partial_{t}^{2} u+\Delta_{x} u\right)-\gamma \partial_{t} u-f(u)=g,\left.\quad u\right|_{\partial \omega}=0, \\
\left.u\right|_{t=0}=u_{0}, \quad(t, x) \in \Omega_{+},
\end{array}\right.
$$

которая интерпретируется и исследуется как эволюционная задача по "времени" $t$. Действительно, если у этой вспомогательной задачи имеется аттрактор (глобальный или траекторный), то, согласно общей теории, он порождается всеми (ограниченными) решениями исходной задачи (7.1) во всем цилиндре, объединение которых мы называем ядром данного эволюционного уравнения (см. раздел 4). Таким образом, изучение решений исходной задачи (7.1) сводится к построению и исследованию аттрактора вспомогательной “динамической системы" (7.3). Однако необходимо заметить, что задача (7.3), как правило, является некорректной и ее решение неединственно даже при идеальных условиях на нелинейную функцию $f$. Это принципиальное отличие от случая эволюционных уравнений является главной трудностью при построении теории аттракторов эллиптических уравнений. Следуя в основном работе [77], мы покажем, как эту трудность можно преодолеть, используя понятие траекторного аттрактора. 
Исследование вспомогательной задачи в полуцилиндре. Мы начнем с вопроса о разрешимости задачи (7.3). Предположим, что $f$ является непрерывной функцией, которая удовлетворяет следующему условию диссипативности:

$$
f(u) \cdot u \geqslant-C+\kappa|u|^{2+\varepsilon}, \quad \forall u \in \mathbb{R}^{k},
$$

где $C, \kappa$ и $\varepsilon$ - некоторые положительные константы, а $v \cdot u$ обозначает стандартное скалярное произведение в $\mathbb{R}^{k}$. Предположим также, что внешняя сила $g$ принадлежит $L^{p}(\omega)$ для некоторого $p>n+1$. Рассмотрим цилиндрическую область $\Omega_{a, b}:=[a, b] \times \omega$. Решением задачи (7.3) называется любая функция $u \in W_{\text {loc }}^{2, p}\left(\Omega_{+}\right)$, т. е. $u \in W^{2, p}\left(\Omega_{0, M}\right)$ для каждого $M>0$, которая удовлетворяет уравнению (7.3) в смысле теории обобщенных функций. Заметим, что пространство $W^{2, p}$ непрерывно вложено в $C$ (и даже в $C^{1}$ ) и поэтому нелинейный оператор $f(u)$ определен корректно. Очевидно, что для разрешимости этой задачи мы должны также потребовать, чтобы начальное условие принадлежало соответствующему пространству следов:

$$
u_{0} \in V_{0}:=W^{2-1 / p, p}(\omega) \cap\left\{\left.u_{0}\right|_{\partial \omega}=0\right\} .
$$

Из следующей теоремы, доказанной в работе [77], вытекает разрешимость вспомогательной задачи в полуцилиндре, а также диссипативная оценка для ее решений.

Теорема 7.1. Пусть выполнены сбормулированные выше условия. Тогда для любого $u_{0} \in V_{0}$ задача (7.3) имеет хотя бы одно решение, причем оно удовлетворяет следующей диссипативной оценке:

$$
\|u\|_{W^{2, p}\left(\Omega_{M, M+1}\right)} \leqslant Q\left(\left\|u_{0}\right\|_{V_{0}}\right) \mathrm{e}^{-\alpha M}+Q\left(\|g\|_{L^{p}}\right),
$$

где положительная константа $\alpha$ и монотонная функиия $Q$ не зависят от M, $u_{0}$ и конкретного выбора решения $и$.

Кратко поясним основную идею вывода диссипативной оценки (7.5). Прежде всего заметим, что любое решение задачи (7.3) является ограниченным, т. е.

$$
\|u\|_{W_{\mathrm{b}}^{2, p}\left(\mathbb{R}_{+} \times \omega\right)}:=\sup _{M>0}\|u\|_{W^{2, p}\left(\Omega_{M, M+1}\right)}<\infty .
$$

Это вытекает из предположения о суперлинейности функции $f$ (условие $\varepsilon>0$ в (7.4), см. [80]). Рассмотрим скалярную функцию $v(t, x):=\mathbf{a} u(t, x) \cdot u(t, x)$. Умножим уравнение (7.3) на функцию $u$ (без интегрирования по $x$ ). Нетрудно убедиться в том, что

$$
\partial_{t}^{2} v+\Delta_{x} v=h_{u}(t, x)
$$

где $h_{u}:=2 \mathbf{a} \partial_{t} u \cdot \partial_{t} u+2 \mathbf{a} \nabla_{x} u \cdot \nabla_{x} u-\gamma \partial_{t} u \cdot u+2 f(u) \cdot u+2 g \cdot u$. Из условия диссипативности функции $f$ следует неравенство

$$
h_{u}(t, x) \geqslant h(x):=-C\left(1+|g(x)|^{2}\right), \quad \forall(t, x) \in \Omega_{+}
$$

для некоторой константы $C$, не зависящей от решения $u$. Поэтому из принципа сравнения для ограниченных решений линейного эллиптического уравнения (7.6) следует оценка

$$
v(t, x) \leqslant w(t, x), \quad \forall(t, x) \in \Omega_{+},
$$


где функция $w$ является ограниченным решением линейной задачи

$$
\partial_{t}^{2} w+\Delta_{x} w=h(x),\left.\quad w\right|_{t=0}=\left.v\right|_{t=0} .
$$

Так как $h \in L^{p / 2}(\omega)$ и $p>n+1$ (на самом деле, достаточно $p>n$, а более аккуратное рассуждение показывает, что для выполнения теоремы достаточно условия $p>(n+1) / 2)$, то линейная задача $(7.8)$ имеет единственное ограниченное решение, которое удовлетворяет аналогу диссипативной оценки (7.5) в пространстве $L^{\infty}\left(\Omega_{0, M}\right)$. Поэтому, согласно оценке $(7.7)$, решение $u$ исходной задачи также удовлетворяет диссипативной оценке в $L^{\infty}$. Теперь искомая оценка в $W^{2, p}$ следует из стандартных внутренних оценок для линейного эллиптического уравнения (после получения оценки в $L^{\infty}$ нелинейный член $f(u)$ может трактоваться как возмущение).

Построение траекторного аттрактора. Отметим, что теорема 7.1 позволяет строить множество сильных решений, которые удовлетворяют диссипативной оценке. Поэтому построение соответствующего траекторного аттрактора проводится согласно общей схеме, изложенной в разделе 4 , и не вызывает дополнительных осложнений.

Пространства $\mathscr{F}_{+}^{\text {loc }}$ и $\mathscr{F}_{+}^{\mathrm{b}}$ определяются формулами

$$
\begin{gathered}
\mathscr{F}_{+}^{\mathrm{loc}}=W_{\mathrm{loc}}^{2, p}\left(\Omega_{+}\right):=L_{\mathrm{loc}}^{p}\left(\mathbb{R}_{+}, W^{2, p}(\omega)\right) \cap W_{\mathrm{loc}}^{2, p}\left(\mathbb{R}_{+}, L^{p}(\omega)\right), \\
\mathscr{F}_{+}^{\mathrm{b}}=W_{\mathrm{b}}^{2, p}\left(\Omega_{+}\right):=L_{\mathrm{b}}^{p}\left(\mathbb{R}_{+}, W^{2, p}(\omega)\right) \cap W_{\mathrm{b}}^{2, p}\left(\mathbb{R}_{+}, L^{p}(\omega)\right),
\end{gathered}
$$

причем

$$
\|u\|_{\mathscr{F}_{+}^{\mathrm{b}}}:=\|u\|_{W_{\mathrm{b}}^{2, p}\left(\mathbb{R}_{+} \times \omega\right)} .
$$

В пространстве $\mathscr{F}_{+}^{\text {loc }}$ вводится (сильная) топология $\Theta_{+}^{\text {loc }}$ сильной локальной сходимости, т. е. $u_{n} \rightarrow u$ при $n \rightarrow \infty$ в топологии $\Theta_{+}^{\text {loc }}$, если

$$
\left\|u_{n}-u\right\|_{W^{2, p}\left(\Omega_{0, M}\right)} \rightarrow 0 \quad(n \rightarrow \infty), \quad \forall M>0 .
$$

По определению, пространство траекторий $\mathscr{K}^{+} \subset \mathscr{F}_{+}^{\mathrm{b}}$, соответствующее задаче (7.3), состоит из всех ее решений, где начальная функция $u_{0}$ является произвольным элементом из $V_{0}$. Тогда из теоремы 7.1 и того факта, что $V_{0}$ есть пространство следов функций из $W^{2, p}\left(\Omega_{0, M}\right)$ на сечении цилиндра, следует, что пространство траекторий $\mathscr{K}^{+}$непусто и инвариантно относительно полугруппы трансляций $\{T(t), t \geqslant 0\}$ вдоль оси цилиндра:

$$
T(t) \mathscr{K}^{+} \subset \mathscr{K}^{+}, \quad t \geqslant 0 .
$$

Напомним, что $(T(t) u)(s, x):=u(t+s, x),(s, x) \in \Omega_{+}$.

Из диссипативной оценки (7.5) следует аналогичная диссипативная оценка для полугруппы $T(t)$ :

$$
\|T(t) u\|_{\mathscr{F}_{+}^{\mathrm{b}}} \leqslant Q\left(\|u\|_{\mathscr{F}_{+}^{\mathrm{b}}}\right) \mathrm{e}^{-\alpha t}+Q\left(\|g\|_{L^{p}}\right) .
$$

Поэтому у полугруппы $\{T(t)\}$ имеется поглощающее множество $\mathscr{P}_{0}$, ограниченное в $\mathscr{F}_{+}^{\mathrm{b}}$ :

$$
\mathscr{P}_{0}:=\left\{u \in \mathscr{K}^{+},\|u\|_{\mathscr{F}_{+}^{\mathrm{b}}} \leqslant 2 Q\left(\|g\|_{L^{p}}\right)\right\} .
$$


В пространстве $\mathscr{K}^{+}$рассматривается введенная выше топология $\Theta_{+}^{\text {loc }}$. Из внутренних оценок для линейного эллиптического уравнения следует, что поглощающее множество

$$
\mathscr{P}_{1}=T(1) \mathscr{P}_{0}
$$

компактно в топологии $\Theta_{+}^{\text {loc }}$. Таким образом, справедлива следующая теорема, подробное доказательство которой можно найти в работе [77].

Теорема 7.2. Пусть выполнены условия теоремы 7.1. Тогда вспомогательная задача (7.3) обладает траекторным аттрактором $\mathfrak{A} \subset \mathscr{K}^{+}$, который притягивает ограниченные в $\mathscr{F}_{+}^{\mathrm{b}}$ подмножества решений в топологии $\Theta_{+}^{\text {loc }}$. Более того, этот аттрактор порождается всеми ограниченными решениями $u \in W_{\mathrm{b}}^{2, p}(\Omega)$ исходной эллиптической задачи в полном иилиндре, m.e.

$$
\mathfrak{A}=\Pi_{+} \mathscr{K},
$$

где $\mathscr{K} \subset W_{\mathrm{b}}^{2, p}(\Omega)$ - ядро исходного уравнения (7.1).

Единственность решений вспомогательной задачи. Хотя в общей ситуации решение задачи (7.3) может быть неединственным, известен весьма важный частный случай, когда единственность имеет место. Это так называемая задача о быстрых бегущих волнах. Этот случай также изучался многими авторами (см. [72], [75], [78]). В частности, в работе [78] единственность решений задачи (7.3) доказана при следующих дополнительных условиях:

$$
f^{\prime}(u) \geqslant-K, \quad \gamma>\gamma_{0}:=\gamma_{0}(\mathbf{a}, K) .
$$

(Предполагается, что $\gamma=\gamma \mathbf{I}$ - скалярная матрица). В этом случае задача (7.3) оказывается эквивалентной эволюционному параболическому уравнению на сечении цилиндра (в пространстве $\left.V_{0}\right)$ :

$$
\partial_{t} u+\left(-\Delta_{x}\right)^{1 / 2} u=F(u),\left.\quad u\right|_{t=0}=u_{0},
$$

где $F$ - нелинейный гладкий оператор, подчиненный линейной части уравнения. По этой причине теория эллиптических аттракторов сводится в этом частном случае к хорошо изученной теории глобальных аттракторов эволюционных уравнений. В частности, при этих условиях аттрактор задачи (7.3) является конечномерным.

\section{Глава III \\ Некоторые вопросы аппроксимации и возмущения траекторных аттракторов}

В данной главе показано, как с помощью метода траекторных аттракторов можно исследовать различные схемы приближения и возмущения в сложных моделях математической физики. Имея ограниченные рамки публикации, мы остановились на четырех задачах, в которых используются траекторные аттракторы. Изложены два метода приближения траекторных аттракторов 
3D-системы Навье-Стокса: метод галёркинских приближений и метод приближения $\alpha$-моделью Лерэ. Кроме того, решена задача о пределе нулевой вязкости для диссипативной 2D-системы Навье-Стокса, который сводится к траекторному аттрактору соответствующей диссипативной 2D-системы Эйлера. В последнем разделе изучается вопрос о сходимости траекторных аттракторов системы реакции-диффузии при стремлении к нулю одного из коэффициентов диффузии. Отметим, что все перечисленные задачи относятся к сложным случаям сингулярного возмущения соответствующих уравнений с частными производными, и их анализ другими методами представляет собой трудные математические проблемы. Другие примеры возмущений рассмотрены в [12], [44], [81], [82]. Некоторые задачи теории усреднения траекторных аттракторов для уравнений с быстро осциллирующими членами изучены в [83].

\section{3D-система Навье-Стокса: приближение траекторными аттракторами галёркинских систем}

В разделе 5 главы II было показано, что 3D-система Навье-Стокса

$$
\partial_{t} u+\nu L u+B(u, u)=g(x), \quad(\nabla, u)=0,\left.\quad u\right|_{\partial \Omega}=0, \quad g(\cdot) \in H,
$$

имеет траекторный аттрактор $\mathfrak{A}$ в пространстве $\mathscr{F}_{+}^{\text {loc }} \cap \Theta_{+}^{\text {loc }}$, где

$$
\mathscr{F}_{+}^{\text {loc }}=L_{2}^{\text {loc }}\left(\mathbb{R}_{+} ; V\right) \cap L_{\infty}^{\text {loc }}\left(\mathbb{R}_{+} ; H\right) \cap\left\{v(\cdot) \mid \partial_{t} v(\cdot) \in L_{4 / 3}^{\text {loc }}\left(\mathbb{R}_{+} ; V^{\prime}\right)\right\}
$$

причем топология $\Theta_{+}^{\text {loc }}$ в этом пространстве определяется локальной слабой сходимостью последовательностей в пространстве

$$
L_{2, \mathrm{w}}^{\mathrm{loc}}\left(\mathbb{R}_{+} ; V\right) \cap L_{\infty, * \mathrm{w}}^{\mathrm{loc}}\left(\mathbb{R}_{+} ; H\right) \cap\left\{v(\cdot) \mid \partial_{t} v(\cdot) \in L_{4 / 3, \mathrm{w}}^{\mathrm{loc}}\left(\mathbb{R}_{+} ; V^{\prime}\right)\right\}
$$

Траекторный аттрактор $\mathfrak{A}$ ограничен в банаховом пространстве

$$
\mathscr{F}_{+}^{\mathrm{b}}=L_{2}^{\mathrm{b}}\left(\mathbb{R}_{+} ; V\right) \cap L_{\infty}^{\mathrm{b}}\left(\mathbb{R}_{+} ; H\right) \cap\left\{v(\cdot) \mid \partial_{t} v(\cdot) \in L_{4 / 3}^{\mathrm{b}}\left(\mathbb{R}_{+} ; V^{\prime}\right)\right\}
$$

с нормой, определенной по формуле (II.5.17).

Траекторный аттрактор системы Галёркина. Пусть $\left\{w_{j}(x)\right\}-$ полная, линейно независимая система функций в пространстве $V$. Обозначим через $H^{m}$ линейную оболочку функций $\left\{w_{j}\right\}, j=1, \ldots, m$, которая является $m$-мерным подпространством $V$. Через $P_{m}$ обозначается ортогональный проектор из пространства $H$ на $H^{m}$ относительно обычного скалярного произведения в $H$ (см. (II.5.3)), $P_{m} H=H^{m}$. Пусть $g_{m}=P_{m} g$. Тогда

$$
\left|g_{m}\right| \leqslant|g| \quad \text { и } \quad g_{m} \rightarrow g(m \rightarrow \infty) \text { сильно в } H .
$$

Отметим, что $|\cdot|$ и $\|\cdot\|$ обозначают нормы в пространствах $H$ и $V$ соответственно. 
Рассматривается галёркинская система порядка $m$ для уравнения (1.1):

$$
\begin{gathered}
\frac{d}{d t} u_{m}+\nu P_{m} L u_{m}+P_{m} B\left(u_{m}, u_{m}\right)=g_{m}, \\
u_{m}(0)=u_{0, m}, \quad u_{0, m}=P_{m} u_{0}, \\
u_{m}(x, t)=\sum_{j=1}^{m} a_{j, m}(t) w_{j}(x), \quad u_{0, m}=\sum_{j=1}^{m} a_{0, j, m} w_{j}(x),
\end{gathered}
$$

где вещественные функции $a_{j, m}(t), j=1, \ldots, m$, абсолютно непрерывны и $u_{0, m} \in H^{m}$. Очевидно, что (1.5), (1.6) является задачей Коши для системы, которая состоит из $m$ обыкновенных дифференциальных уравнений относительно неизвестных вещественных функций $a_{j, m}(t)$ с начальными условиями $a_{j, m}(0)=a_{0, j, m}$ при $j=1, \ldots, m$. Как уже отмечалось в разделе 5 главы II, эта задача имеет единственное решение в пространстве $C^{1, \text { loc }}\left(\mathbb{R}_{+} ; H^{m}\right)$. При этом выполнено энергетическое тождество

$$
\frac{1}{2} \frac{d}{d t}\left|u_{m}(t)\right|^{2}+\nu\left\|u_{m}(t)\right\|^{2}=\left(g, u_{m}(t)\right), \quad t \geqslant 0 .
$$

Из (1.7) вытекают основные априорные оценки для задачи (1.5), (1.6):

$$
\begin{gathered}
\left|u_{m}(t)\right|^{2} \leqslant\left|u_{0}\right|^{2} \mathrm{e}^{-\nu \lambda_{1} t}+\frac{|g|^{2}}{\nu^{2} \lambda_{1}^{2}}, \\
\nu \int_{t}^{t+1}\left\|u_{m}(s)\right\|^{2} d s \leqslant\left|u_{0}\right|^{2} \mathrm{e}^{-\nu \lambda_{1} t}+\frac{|g|^{2}}{\nu^{2} \lambda_{1}^{2}}+\frac{|g|^{2}}{\nu \lambda_{1}},
\end{gathered}
$$

из которых выводится глобальная разрешимость системы ОДУ (1.5), (1.6).

Воспользуемся схемой построения траекторного аттрактора системы ОДУ, изложенной в разделе 1 главы II.

Обозначим через $\mathscr{K}_{m}^{+}$множество всех решений $u_{m}(s), s \geqslant 0$, уравнения (1.5) (с произвольными начальными условиями), которые принадлежат пространству $C^{1, \text { loc }}\left(\mathbb{R}_{+} ; H^{m}\right)$. Здесь, как обычно, переменная $s \geqslant 0$ обозначает время.

В качестве пространств $\mathscr{F}_{+}^{\text {loc, } m}$ и $\mathscr{F}_{+}^{\mathrm{b}, m}$ берутся пространства

$$
\mathscr{F}_{+}^{\text {loc }, m}:=C^{1, \mathrm{loc}}\left(\mathbb{R}_{+} ; H^{m}\right), \quad \mathscr{F}_{+}^{\mathrm{b}, m}:=C^{1, \mathrm{~b}}\left(\mathbb{R}_{+} ; H^{m}\right) .
$$

Норма в $\mathscr{F}_{+}^{\mathrm{b}, m}$ равна

$$
\|v\|_{\mathscr{F}_{+}^{\mathrm{b}, m}}:=\sup _{s \geqslant 0}\|v(s)\|_{H^{m}}+\sup _{s \geqslant 0}\left\|\partial_{t} v(s)\right\|_{H^{m}} .
$$

Отметим, что все нормы в конечномерном пространстве $H^{m}$ эквивалентны и можно, например, положить $\|v\|_{H^{m}}:=|v|$. Из оценки (1.8) и из уравнения (1.5) вытекает, что $\mathscr{K}_{m}^{+} \subset \mathscr{F}_{+}^{\mathrm{b}, m}$.

Через $\Theta_{+}^{\text {loc, } m}$ обозначается топология в $\mathscr{F}_{+}^{\text {loc, } m}$, в которой, по определению, последовательность $\left\{v_{n}(\cdot)\right\} \subset \mathscr{F}_{+}^{\text {loc, } m}$ сходится к $v(\cdot) \in \mathscr{F}_{+}^{\text {loc, } m}$ при $n \rightarrow \infty$, если для любого $M>0$

$$
\max _{s \in[0, M]}\left(\left\|v_{n}(s)-v(s)\right\|_{H^{m}}+\left\|\partial_{t} v_{n}(s)-\partial_{t} v(s)\right\|_{H^{m}}\right) \rightarrow 0 \quad(n \rightarrow \infty) .
$$


Рассмотрим трансляционную полугруппу $\{T(t)\}$ на $\mathscr{K}_{m}^{+}$. Из оценки (1.8) и из уравнения (1.5) следует, что при любом фиксированном $m$ множество

$$
\mathscr{P}_{m}=\left\{u_{m} \in \mathscr{K}_{m}^{+} \mid\left\|u_{m}\right\|_{\mathscr{F}_{+}^{\mathrm{b}, m}} \leqslant R_{m}\right\}
$$

является поглощающим для полугруппы $\left.\{T(t)\}\right|_{\mathscr{K}_{m}^{+}}$, если $R_{m}$ достаточно велико (см. [12]). Множество $\mathscr{P}_{m}$, очевидно, ограничено в $\mathscr{F}_{+}^{\mathrm{b}, m}$. Легко проверить, что $\mathscr{P}_{m}$ компактно в $\Theta_{+}^{\text {loc, } m}$. (См. в главе II утверждения 1.1 и 1.5.) Из общих теорем о траекторных аттракторах для систем ОДУ, сформулированных в разделе 1 главы II, получаем следующий результат.

Теорема 1.1. Трансляиионная полугруппа $\{T(t)\}$, действующая на $\mathscr{K}_{m}^{+}$, обладает траекторным аттрактором $\mathfrak{A}_{m}$ (в топологии $\Theta_{+}^{\text {loc, } m}$ пространства $\mathscr{F}_{+}^{\text {loc, } m}=C^{1, \text { loc }}\left(\mathbb{R}_{+} ; H^{m}\right)$, а ограниченные множества из $\mathscr{K}_{m}^{+}$берутся по норме пространства $\left.\mathscr{F}_{+}^{\mathrm{b}, m}\right)$. Множество $\mathfrak{A}_{m}$ ограничено в $\mathscr{F}_{+}^{\mathrm{b}, m}$, компактно в $\Theta_{+}^{\mathrm{loc}, m}$ и связано с ядром $\mathscr{K}_{m}$ галёркинской системы (1.5) формулой

$$
\mathfrak{A}_{m}=\Pi_{+} \mathscr{K}_{m}(0) .
$$

ЗАмечание 1.1. Множество $\mathfrak{A}_{m}$ ограничено в $C^{1, \mathrm{~b}}\left(\mathbb{R}_{+} ; H\right)$, но соответствующие оценки не являются равномерными по порядку $m$ галёркинской системы! Это связано с тем, что при выводе оценок для нормы функции $\partial_{t} u_{m}(t), t \geqslant 0$, в пространстве $C^{\mathrm{b}}\left(\mathbb{R}_{+} ; H\right)$ с помощью $(1.8),(1.9)$ справа в неравенствах возникают величины, зависящие от $m$. По этой же причине нельзя получить априорные оценки в $H$ для производных по $t$ решений 3D-системы Навье-Стокса, которые строятся по методу Галёркина.

Напомним, что система Галёркина (1.5) является системой обыкновенных дифференциальных уравнений в конечномерном пространстве $H^{m}$. Из однозначной глобальной разрешимости этой системы следует, что она порождает в $H^{m}$ конечномерную динамическую систему или полугруппу:

$$
S(t): H^{m} \rightarrow H^{m}, \quad \forall u_{0, m} \in H^{m} \quad S(t) u_{0, m}=u_{m}(t), \quad t \geqslant 0 .
$$

Из результатов главы I следует существование у этой полугруппы в $H^{m}$ глобального аттрактора $\mathscr{A}_{m}$. Имеется очевидная связь между множествами $\mathscr{A}_{m}$ и $\mathfrak{A}_{m}$ :

$$
\mathscr{A}_{m}=\mathfrak{A}_{m}(0) .
$$

Отметим, что траекторное пространство $\mathscr{K}_{m}^{+}$галёркинской системы (1.5) можно рассматривать и изучать в слабом объемлющем пространстве $\mathscr{F}_{+}^{\text {loc }}$ (см. (1.2)), которое ассоциировалось в разделе 5 главы II с 3D-системой НавьеСтокса. При этом все получающиеся оценки будут равномерными по $m$ - порядку галёркинской системы (см. [12]).

СлеДСтвиЕ 1.1. Траекторный аттрактор $\mathfrak{A}_{m}$ из теоремы 1.1 притягивает ограниченные множества из $\mathscr{K}_{m}^{+}$в топологии $\Theta_{+}^{\text {loc }}$. Более того, множества $\mathfrak{A}_{m}$ являются равномерно ограниченными в пространстве $\mathscr{F}_{+}^{\mathrm{b}}($ см. (1.3)), т.е. справедливо неравенство

$$
\left\|u_{m}(\cdot)\right\|_{\mathscr{F}_{+}^{\mathrm{b}}} \leqslant R, \quad \forall u_{m} \in \mathfrak{A}_{m}, \quad \forall m \in \mathbb{N},
$$

где величина $R$ не зависит от $m$. 
Сходимость траекторных аттракторов галёркинской системы. Изучим вопрос о приближении траекторного аттрактора $\mathfrak{A} 3 \mathrm{D}$-системы НавьеСтокса с помощью траекторных аттракторов $\mathfrak{A}_{m}$ галёркинских систем (1.5). Справедлива следующая теорема, являющаяся основной в данном разделе.

Теорема 1.2. Траекторный аттрактор $\mathfrak{A}_{m}$ галёркинской системы (1.5) сходится при $m \rightarrow \infty \kappa$ траекторному аттрактору $\mathfrak{A}$ 3D-системъ НавъеСтокса в топологии $\mathscr{F}_{+}^{\text {loc }} \cap \Theta_{+}^{\text {loc }}$. Точнее, для любой окрестности $\mathscr{O}(\mathfrak{A})$ множества $\mathfrak{A}$ в топологии $\Theta_{+}^{\text {loc }}$ найдется такое число $N=N(\mathscr{O})$, что

$$
\mathfrak{A}_{m} \subseteq \mathscr{O}(\mathfrak{A}), \quad \forall m \geqslant N .
$$

Отсюда, в частности, следует, что при любом $M>0$

$$
\operatorname{dist}_{L_{2}\left(0, M ; H^{1-\delta}\right)}\left(\mathfrak{A}_{m}, \mathfrak{A}\right) \rightarrow 0 \quad(m \rightarrow \infty), \quad \delta>0 .
$$

ДокАЗАтЕЛЬСтво. Проводится от противного. Предположим, что утверждение теоремы неверно. Тогда найдется окрестность $\mathscr{O}(\mathfrak{A})$ в $\Theta_{+}^{\text {loc }}$, для которой (1.14) не имеет места. Следовательно, существует последовательность $\left\{u_{m^{\prime}}\right\}, u_{m^{\prime}} \in \mathfrak{A}_{m^{\prime}}$ такая, что

$$
u_{m^{\prime}} \notin \mathscr{O}(\mathfrak{A}) \quad\left(m^{\prime} \rightarrow \infty\right) .
$$

В силу (1.13)

$$
\left\|u_{m^{\prime}}(\cdot)\right\|_{\mathscr{F} \mathrm{b}} \leqslant R
$$

Напомним, что любой шар в $\mathscr{F}_{+}^{\mathrm{b}}$ слабо компактен в топологии $\Theta_{+}^{\text {loc }}$. Поэтому, переходя, если необходимо, к подпоследовательности, можно считать, что $\left\{u_{m^{\prime}}(\cdot)\right\}$ сходится к некоторой функции $u(\cdot) \in \mathscr{F}_{+}^{\mathrm{b}}$ :

$$
u_{m^{\prime}}(\cdot) \rightarrow u(\cdot) \quad\left(m^{\prime} \rightarrow \infty\right) \quad \text { в топологии } \Theta_{+}^{\text {loc }} .
$$

Как известно, пределами галёркинских последовательностей являются слабые решения системы Навье-Стокса, т. е. $u(\cdot) \in \mathscr{K}^{+}$. Можно легко проверить, что $u(\cdot) \in \mathfrak{A}$ (для этого необходимо воспользоваться представлениями траекторных аттракторов (II.5.25) и (1.11), см. подробности в [12]). С другой стороны, из $(1.15)$ вытекает, что $u(\cdot) \notin \mathscr{O}(\mathfrak{A})$. Противоречие. Теорема доказана.

В заключение получим результат о приближении слабого глобального аттрактора $\mathscr{A}$ 3D-системы Навье-Стокса, который был построен в разделе 5 главы II.

СледСтвие 1.2. Глобальный аттрактор $\mathscr{A}_{m}$ системы Галёркина (1.5) сходится при $m \rightarrow \infty \kappa$ слабому глобальному аттрактору $\mathscr{A}$ системы НавъеСтокса в слабой топологии пространства $H$.

Для доказательства достаточно воспользоваться тождеством (1.11).

Аналогичные результаты имеют место для аттракторов галёркинских приближений диссипативной системы Эйлера, систем реакции-диффузии, волновых уравнений с диссипацией, рассмотренных в главе II, а также для других уравнений и систем математической физики. 


\section{3D-система Навье-Стокса: аппроксимация траекторными аттракторами $\alpha$-модели Лерэ}

В последние годы большую популярность приобрели так называемые $\alpha$-модели 3D-системы Навье-Стокса. Эти модели представляют собой различные регуляризованные приближения 3D-системы Навье-Стокса, зависящие от малого параметра $\alpha$, в которых неизвестная функция скорости $v$ в ряде слагаемых заменена на более гладкую вектор-функцию $u$, связанную с $v$ эллиптической системой $v=u-\alpha^{2} \Delta u$. В настоящем разделе рассматривается $\alpha$-модель Лерэ. Другая популярная $\alpha$-модель называется системой Камассы-Холма с вязкостью. Она изучалась, например, в [84]-[86]. Параметр $\alpha$ имеет определенный физический смысл. Исследовать $\alpha$-модели существенно проще и численно, и аналитически, так как, эти сглаженные системы являются корректными системами уравнений. Кроме того, имеется определенная схожесть решений $\alpha$-моделей с эмпирическими данными, которые были получены для некоторых реальных турбулентных течений (см. [87], [88]). Однако остается весьма актуальным вопрос о связи долговременных эволюций $\alpha$-моделей с предельной динамикой точной системы Навье-Стокса. В настоящем разделе обоснование этой связи для $\alpha$-модели Лерэ осуществляется методами траекторных аттракторов. Аналогичное исследование для системы Камассы-Холма было проделано в [89].

Трехмерная $\alpha$-модель Лерэ вязкой несжимаемой жидкости. Рассматривается следующая система уравнений при периодических граничных условиях:

$$
\left\{\begin{array}{l}
\partial_{t} v-\nu \Delta v+(u, \nabla) v+\nabla p=g(x), \quad(\nabla, v)=0 \\
v=u-\alpha^{2} \Delta u, \quad x=\left(x_{1}, x_{2}, x_{3}\right) \in \mathbb{T}^{3}
\end{array}\right.
$$

Здесь $\mathbb{T}^{3}:=[\mathbb{R} \bmod 2 \pi \ell]^{3}$ - трехмерный тор. Неизвестными являются векторфункции $v=v(x, t)=\left(v^{1}, v^{2}, v^{3}\right)$ и $u=u(x, t)=\left(u^{1}, u^{2}, u^{3}\right)$, а также скалярная функция $p=p(x, t)$. Вектор-функция $g=g(x)=\left(g^{1}, g^{2}, g^{3}\right)$ считается известной. Предполагается, что функции $v, u, p$ и $g$ являются периодическими с периодом $2 \pi \ell$ по $x_{i}, i=\overline{1,2,3}$, и их среднее значение по $x$ равно нулю.

Заметим, что при $\alpha=0$ функция $v$ равна $u$ и система (2.1) совпадает с $3 \mathrm{D}$-системой Навье-Стокса на торе $\mathbb{T}^{3}$. При $\alpha>0$ система $(2.1)$ называется $\alpha$-моделью Лерэ (см. [84]).

Перепишем систему (2.1) в эквивалентной форме, применив к обеим частям уравнений оператор проектирования $P$ на пространство $H$ (см. раздел 5 главы II). Напомним, что пространства $H$ и $V$ являются замыканиями в $\left[L_{2}\left(\mathbb{T}^{3}\right)\right]^{3}$ и $\left[H^{1}\left(\mathbb{T}^{3}\right)\right]^{3}$ соответственно линейного подпространства тригонометрических $3 \mathrm{D}$-полиномов с нулевой дивергенцией и с нулевым средним. В результате получим следующую систему:

$$
\begin{gathered}
\partial_{t} v+\nu L v+B(u, v)=g(x), \\
v=u+\alpha^{2} L u
\end{gathered}
$$


в которой $L:=-P \Delta$ обозначает оператор Стокса и $B(u, v):=P(u, \nabla) v$. Как известно, в задаче с периодическими граничными условиями оператор Стокса имеет вид $L v=-\Delta v$ при любом $v \in H$.

Линейное уравнение (2.3) имеет единственное решение $u \in H^{2} \cap V$, причем

$$
\|u\|_{H^{2}}:=|L u| \leqslant \frac{1}{\alpha^{2}}|v|, \quad \forall v \in H,
$$

где $H^{2}:=\left[H^{2}\left(\mathbb{T}^{3}\right)\right]^{3}$. Напомним, что нормы в пространствах $H$ и $V$ обозначаются $|\cdot|$ и ||$\cdot||$ соответственно. Из теоремы вложения Соболева следует, что $H^{2}\left(\mathbb{T}^{3}\right) \subset L_{\infty}\left(\mathbb{T}^{3}\right)$. В частности, справедливо неравенство

$$
\|u\|_{\left[L_{\infty}\left(\mathbb{T}^{3}\right)\right]^{3}} \leqslant C(\alpha)\left|u+\alpha^{2} L u\right|=C(\alpha)|v|, \quad \forall u \in H^{2} \cap V,
$$

где $v=u+\alpha^{2} L u$. В [84] показано, что $C(\alpha) \leqslant \frac{5}{4}+\frac{8}{5}\left(\frac{\ell}{c_{1} \alpha}\right)^{3 / 2}$, где $c_{1}>0-$ некоторая абсолютная константа. Отметим, что $C(\alpha) \rightarrow+\infty$ при $\alpha \rightarrow 0+$. Из (2.5) следует, что

$$
|B(u, v)| \leqslant|(u, \nabla) v| \leqslant c\|u\|_{\left[L_{\infty}\left(\mathbb{T}^{3}\right)\right]^{3}}\|v\| \leqslant C_{1}(\alpha)|v|\|v\|, \quad \forall v \in V,
$$

где $v=u+\alpha^{2} L u$.

Изучаются слабые решения $v(x, t)$ системы $(2.2)-(2.3)$, принадлежащие пространству $L_{2}(0, M ; V) \cap L_{\infty}(0, M ; H)$. Соответствующая функция $u(x, t)$, которая получается при решении уравнения (2.3), принадлежит пространству $L_{\infty}\left(0, M ; H^{2}\right)$. Тогда из $(2.6)$ заключаем, что

$$
B(u(\cdot), v(\cdot)) \in L_{2}(0, M ; H) .
$$

Кроме того, $L v(\cdot) \in L_{2}\left(0, M ; V^{\prime}\right)$, и в силу (2.7) из уравнения $(2.2)$ находим, что

$$
\partial_{t} v(\cdot) \in L_{2}\left(0, M ; V^{\prime}\right)
$$

Изучается система (2.2)-(2.3) при начальном условии

$$
\left.v\right|_{t=0}=v_{0}(x) \in H .
$$

Имеет место следующий результат о существовании и единственности слабого решения задачи Коши для $\alpha$-модели Лерэ.

УтВеРЖДЕНИЕ 2.1. Пусть $\alpha>0, g \in V^{\prime}, v_{0} \in H$. Тогда задача (2.2), (2.3), (2.9) имеет и притом единственное решение $v(\cdot) \in L_{2}(0, M ; V) \cap L_{\infty}(0, M ; H)$, $u(\cdot) \in L_{\infty}\left(0, M ; H^{2}\right)$ такое, что $\partial_{t} v(\cdot) \in L_{2}\left(0, M ; V^{\prime}\right)$ и функиия $v(\cdot)$ удовлетворяет энергетическому тождеству

$$
\frac{1}{2} \frac{d}{d t}|v(t)|^{2}+\nu\|v(t)\|^{2}=(g, v(t))
$$

в котором скалярная функиия $|v(t)|^{2}, t \in[0, M]$, абсолютно непрерывна, т.е. ее производная по времени имеет классический смысл и равенство (2.10) въполнено при почти всех $t \in[0, M]$. Кроме того, $v \in C([0, M] ; H)$. (Очевидно, что эта задача однозначно разрешима и на всей полупрямой $\{t \geqslant 0\}=\mathbb{R}_{+}$.) 
Доказательство проводится стандартно. Решение строится по методу Галёркина (см. [84]). Единственность слабого решения в указанном классе функций доказывается обычным методом (см., например, [1], [26], [27]). При выводе энергетического тождества (2.10) уравнение (2.2) умножается скалярно в $H$ на функцию $v(\cdot) \in L_{2}(0, M ; V)$. Затем используется свойство ортогональности билинейной формы

$$
(B(u, v), v)=0, \quad \forall u, v \in V,
$$

а также следующее известное соотношение из [26]:

$$
2\left(\partial_{t} v, v\right)=\frac{d}{d t}|v(t)|^{2} \quad \text { для почти всех } t \in[0, M] .
$$

Из энергетического тождества (2.10) следует, что слабое решение $v(t)$ системы (2.2)-(2.3) удовлетворяет следующим неравенствам:

$$
\begin{gathered}
|u(t)|^{2} \leqslant|v(t)|^{2} \leqslant|v(0)|^{2} \mathrm{e}^{-\nu \lambda_{1} t}+\frac{\|g\|_{V^{\prime}}^{2}}{\nu^{2} \lambda_{1}} \\
\nu \int_{t}^{t+1}\|u(s)\|^{2} d s \leqslant \nu \int_{t}^{t+1}\|v(s)\|^{2} d s \leqslant|v(0)|^{2} \mathrm{e}^{-\nu \lambda_{1} t}+\frac{\|g\|_{V^{\prime}}^{2}}{\nu^{2} \lambda_{1}}+\frac{\|g\|_{V^{\prime}}^{2}}{\nu}
\end{gathered}
$$

где $u(t)$ - решение $(2.3)$ и $\lambda_{1}$ - первое собственное значение оператора $L$.

Отметим, что правые части в оценках (2.11) и (2.12) не зависят от $\alpha$.

В [84] доказано следующее свойство сглаживания для решений системы (2.2)-(2.3). Если $g \in H$, то решение $v(t)$ системы (2.2)-(2.3) удовлетворяет неравенству

$$
t\|v(t)\|^{2}+\nu \int_{0}^{t} s|A u(s)|^{2} d s \leqslant C_{2}(\alpha, t,|v(0)|,|g|), \quad \forall t \geqslant 0,
$$

где $C_{2}\left(\alpha, z, R_{1}, R_{2}\right)$ - некоторая положительная монотонно возрастающая функция относительно $z, R_{1}$ и $R_{2}$ при фиксированном $\alpha>0$.

Рассмотрим при $\alpha>0$ полугруппу $\left\{S_{\alpha}(t)\right\}$, которая действует в $H$ по формуле $S_{\alpha}(t) v_{0}=v(t)$ при любом $v_{0} \in H$, и $v(t)$ - решение задачи $(2.2),(2.3),(2.9)$. Из оценки (2.11) следует, что полугруппа $\left\{S_{\alpha}(t)\right\}$ имеет ограниченное (в $\left.H\right)$ поглощающее множество $P_{0}=\left\{v|| v \mid \leqslant R_{0}\right\}$, где $R_{0}^{2}=2\|g\|_{V^{\prime}}^{2} /\left(\nu^{2} \lambda_{1}\right)$. Множество $P_{1}=S_{\alpha}(1) P_{0}$ также является поглощающим, а в силу $(2.13)$, оно ограничено в $V$ и, следовательно, компактно в $H$. Легко проверить, что полугруппа $\left\{S_{\alpha}(t)\right\}$ непрерывна в $H$. Тогда применима теорема 1.1 главы I, в силу которой $\alpha$-модель Лерэ имеет глобальный аттрактор $\mathscr{A}_{\alpha}$ при $\alpha>0$, причем множество $\mathscr{A}_{\alpha}$ компактно в $H$, строго инвариантно и имеет место соотношение

$$
\operatorname{dist}_{H}\left(S_{\alpha}(t) B, \mathscr{A}_{\alpha}\right) \rightarrow 0 \quad \text { при } t \rightarrow+\infty
$$

для любого ограниченного множества $B \subset H$ начальных данных. Кроме того, $\mathscr{A}_{\alpha}$ ограничено в $V$.

Далее изучается поведение решений $\alpha$-модели Лерэ при $\alpha \rightarrow 0+$, а также устанавливается связь с траекторным аттрактором 3D-системы Навье-Стокса. 
Сходимость решений $\alpha$-модели Лерэ к решениям 3D-системы Навье-Стокса. Пространства $\mathscr{F}_{+}^{\text {loc }}$ и $\mathscr{F}_{+}^{\mathrm{b}}$ определяются формулами $(1.2)$ и (1.3), в которых $H$ и $V$ - пространства, построенные по оператору Стокса $L$ с периодическими граничными условиями.

Вводится пространство $\mathscr{K}_{\alpha}^{+}$траекторий $\alpha$-системы Лерэ. По определению, $\mathscr{K}_{\alpha}^{+}$является объединением всех слабых решений $v(s):=v(x, s), s \geqslant 0$, системы (2.2)-(2.3) при любых начальных условиях $v_{0} \in H$. Как обычно, переменная $s=t$ обозначает время.

Рассмотрим полугруппу трансляций $\{T(t)\}$ на пространстве траекторий $\mathscr{K}_{\alpha}^{+}$. Легко проверить, что

$$
T(t) \mathscr{K}_{\alpha}^{+} \subseteq \mathscr{K}_{\alpha}^{+}, \quad \forall t \geqslant 0 .
$$

Имеет место следующее важное свойство пространства $\mathscr{K}_{\alpha}^{+}$, выведенное в [90] из неравенств (2.11) и (2.12).

УтвеРЖДЕНИЕ 2.2. Пусть $g \in V^{\prime}$. Тогда любое решение $v(s), s \geqslant 0$, системъ (2.2)-(2.3) принадлежит $\mathscr{F}_{+}^{\mathrm{b}} u$

$$
\|T(t) v(\cdot)\|_{\mathscr{F}_{+}^{\mathrm{b}}} \leqslant C|v(0)|^{2} \mathrm{e}^{-\beta t}+R_{1}^{2}, \quad \forall t \geqslant 0
$$

где $C$ и $R_{1}$ не зависят от $\alpha, C$ зависит от $\lambda_{1} u \nu$, a $R_{1}$ - от $\lambda_{1}, \nu u\|g\|_{V^{\prime}}$.

В пространстве $\mathscr{F}_{+}^{\text {loc }}$ задается топология $\Theta_{+}^{\text {loc }}$, которая определяется с помощью слабой и *-слабой сходимости последовательностей в соответствующих банаховых пространствах (см. раздел 5 главы II).

Рассматривается 3D-система Навье-Стокса с периодическими граничными условиями на торе $\mathbb{T}^{3}$, которая формально получается из уравнений $(2.2),(2.3)$ при $\alpha=0$. Для этой системы строится пространство траекторий $\mathscr{K}_{0}^{+}$подобно тому, как это делалось в разделе 5 главы II для 3D-системы Навье-Стокса с нулевыми граничными условиями. Напомним, что $\mathscr{K}_{0}^{+}$состоит из всех слабых решений 3D-системы Навье-Стокса, которые удовлетворяют энергетическому неравенству (II.5.11).

Следующее утверждение является ключевым при доказательстве сходимости решений $\alpha$-модели Лерэ при $\alpha \rightarrow 0+$ (см. [90]).

УТвеРЖДЕНИЕ 2.3. Пусть имеется последовательность $v_{\alpha_{n}}(s) \in \mathscr{K}_{\alpha_{n}}^{+}$, $n \in \mathbb{N}$, такал, что $\left\{v_{\alpha_{n}}(\cdot)\right\}$ ограничена по норме $\mathscr{F}_{+}^{\mathrm{b}}$, причем $v_{\alpha_{n}}(\cdot) \rightarrow v(\cdot)$ в топологии $\Theta_{+}^{\text {loc }}$ u $\alpha_{n} \rightarrow 0+n р и ~ n \rightarrow \infty$. Тогда $v(\cdot)$ является слабым решением 3D-системы Навъе-Стокса, и при этом $v \in \mathscr{K}_{0}^{+}$, m.е. $v(s)$ удовлетворяет энергетическому неравенству (II.5.11) при $s \geqslant 0$.

ЗАмечАниЕ 2.1. Пусть последовательность $v_{\alpha_{n}}(s) \in \mathscr{K}_{\alpha_{n}}^{+}$ограничена в $\mathscr{F}_{+}^{\mathrm{b}}$ : $\left\|v_{\alpha_{n}}(\cdot)\right\|_{\mathscr{F}_{+}^{\mathrm{b}}} \leqslant R$. Тогда, если $\alpha_{n} \rightarrow 0+(n \rightarrow \infty)$, то найдутся подпоследовательность $\left\{n^{\prime}\right\} \subset\{n\}$ и функция $v \in \mathscr{K}_{0}^{+}$такие, что $v_{\alpha_{n^{\prime}}}(\cdot) \rightarrow v(\cdot)\left(n^{\prime} \rightarrow \infty\right)$ в топологии $\Theta_{+}^{\text {loc }}$. 
Сходимость ограниченных множеств траекторий $\alpha$-модели Лерэ к траекторному аттрактору 3D-системы Навье-Стокса. Обозначим через $\mathfrak{A}_{0}$ траекторный аттрактор 3D-системы Навье-Стокса

$$
\partial_{t} v+\nu A v+B(v, v)=g(x) .
$$

(См. раздел 5 главы II при нулевых граничных условиях. Случай периодических граничных условий полностью аналогичен.) Напомним, что множество $\mathfrak{A}_{0}$ ограничено в $\mathscr{F}_{+}^{\mathrm{b}}$ и компактно в топологии $\Theta_{+}^{\text {loc }}$.

Пусть $\mathfrak{B}_{\alpha}=\left\{v_{\alpha}(x, s), s \geqslant 0\right\}, 0<\alpha \leqslant 1$, обозначает равномерно (по $\alpha \in(0,1])$ ограниченное в $\mathscr{F}_{+}^{\mathrm{b}}$ семейство решений (траекторий) $\alpha$-модели Лерэ:

$$
\partial_{t} v_{\alpha}+\nu A v_{\alpha}+B\left(u_{\alpha}, v_{\alpha}\right)=g(x), \quad v_{\alpha}=u_{\alpha}+\alpha^{2} A u_{\alpha},
$$

т. е. $\mathfrak{B}_{\alpha} \subset \mathscr{K}_{\alpha}^{+}$при $\alpha \in(0,1]$ и

$$
\left\|v_{\alpha}\right\|_{\mathscr{F}_{+}^{\mathrm{b}}}=\left\|v_{\alpha}\right\|_{L_{2}^{\mathrm{b}\left(\mathbb{R}_{+} ; V\right)}}+\left\|v_{\alpha}\right\|_{L_{\infty}^{\mathrm{b}}\left(\mathbb{R}_{+} ; H\right)}+\left\|\partial_{t} v_{\alpha}\right\|_{L_{4 / 3}^{\mathrm{b}}\left(\mathbb{R}_{+} ; V^{\prime}\right)} \leqslant R, \quad \forall v_{\alpha} \in \mathfrak{B}_{\alpha} .
$$

Обозначим через $\mathscr{K}_{0}$ ядро уравнения (2.15). Напомним, что $\mathscr{K}_{0}$ состоит из объединения всех ограниченных (в $\mathscr{F}^{\mathrm{b}}$ ) полных решений $\{v(s), s \in \mathbb{R}\}$ уравнения (2.15), которые удовлетворяют энергетическому неравенству. В разделе 5 главы II было показано, что $\mathfrak{A}_{0}=\Pi_{+} \mathscr{K}_{0}$. Сформулируем основную теорему.

Теорема 2.1. Пусть $\mathfrak{B}_{\alpha}=\left\{v_{\alpha}(x, s), s \geqslant 0\right\}, 0<\alpha \leqslant 1$, - любое ограниченное множество траекторий $\alpha$-модели Лерэ (2.16), удовлетворяющее неравенству

$$
\left\|\mathfrak{B}_{\alpha}\right\|_{\mathscr{F}_{+}^{\mathrm{b}}} \leqslant R, \quad 0<\alpha \leqslant 1 .
$$

Тогда сдвинутые множества решений $\left\{T(t) \mathfrak{B}_{\alpha}\right\}$ сходятся $\kappa$ траекторному aттрактору $\mathfrak{A}_{0}=\Pi_{+} \mathscr{K}_{0} 3 \mathrm{D}$-системь Навъе-Стокса $(2.15)$ в топологии $\Theta_{+}^{\text {loc }}$ npu $t \rightarrow+\infty u \alpha \rightarrow 0+:$

$$
T(t) \mathfrak{B}_{\alpha} \rightarrow \mathfrak{A}_{0} \quad{ }_{\text {в }} \Theta_{+}^{\text {loc }} \text { npu } t \rightarrow+\infty, \alpha \rightarrow 0+.
$$

Доказательство приведено в [90]. На самом деле, там доказано более сильное утверждение.

СлеДСТВИЕ 2.1. При выполнении условий теоремы 2.1 множество $T(t) \mathfrak{B}_{\alpha}$ сходится к ядру $\mathscr{K}_{0}$ уравнения (2.15) в слабой топологии $\Theta_{-M, м}$ при $h \rightarrow+\infty$ u $\alpha \rightarrow 0+$ при каждом $M>0$. (Топология $\Theta_{-M, M}$ определяет топологию индуктивного предела $\Theta^{\text {loc }}$ в $\left.\mathscr{F}^{\mathrm{loc}}.\right)$

Напомним, что при каждом $M>0$ слабая топология $\Theta_{-M, M}$ сильнее топологии сходимости по норме в пространстве $L_{2}\left(-M, M ; H^{1-\delta}\right)$, а также в пространстве $C\left([-M, M] ; H^{-\delta}\right)$ при любом $0<\delta \leqslant 1$.

СлЕДСТвИЕ 2.2. При любом фиксированном $M>0$ для любого множества $\mathfrak{B}_{\alpha} \subset \mathscr{K}_{\alpha}^{+}$, равномерно ограниченного в $\mathscr{F}_{+}^{\mathrm{b}}$, справедливы следующие предельные соотношения при $t \rightarrow+\infty, \alpha \rightarrow 0+$ :

$$
\begin{aligned}
\operatorname{dist}_{L_{2}\left(-M, M ; H^{1-\delta}\right)}\left(T(t) \mathfrak{B}_{\alpha}, \mathscr{K}_{0}\right) \rightarrow 0+ \\
\operatorname{dist}_{C\left([-M, M] ; H^{-\delta}\right)}\left(T(t) \mathfrak{B}_{\alpha}, \mathscr{K}_{0}\right) \rightarrow 0+.
\end{aligned}
$$


Теорему 2.1 можно использовать для изучения поведения траекторных и глобальных аттракторов $\alpha$-модели Лерэ при $\alpha \rightarrow 0+$.

Рассмотрим траекторное пространство $\mathscr{K}_{\alpha}^{+}, \alpha>0$, системы $(2.2)-(2.3)$, построенное выше. На $\mathscr{K}_{\alpha}^{+}$действует трансляционная полугруппа $\{T(t)\}$. Легко проверить, что $\mathscr{K}_{\alpha}^{+}$замкнуто в топологии $\Theta_{+}^{\text {loc }}$. Из утверждения 2.2 следует, что $\mathscr{K}_{\alpha}^{+} \subset \mathscr{F}_{+}^{\mathrm{b}}$, и, кроме того, у полугруппы $\{T(t)\}$ на $\mathscr{K}_{\alpha}^{+}$есть поглощающее множество, ограниченное в $\mathscr{F}_{+}^{\mathrm{b}}$ и компактное в топологии $\Theta_{+}^{\text {loc }}$. Поэтому существует траекторный аттрактор $\mathfrak{A}_{\alpha} \alpha$-модели Лерэ при $\alpha>0$, т. е. множество $\mathfrak{A}_{\alpha} \subset \mathscr{K}_{\alpha}^{+}$, ограниченное в $\mathscr{F}_{+}^{\mathrm{b}}$, компактное в $\Theta_{+}^{\text {loc }}$ и такое, что $T(t) \mathfrak{A}_{\alpha}=\mathfrak{A}_{\alpha}$ при всех $t \geqslant 0$, причем $T(t) \mathfrak{B}_{\alpha} \rightarrow \mathfrak{A}_{\alpha}$ в топологии $\Theta_{+}^{\text {loc } п р и ~} t \rightarrow+\infty$ для любого ограниченного множества $\mathfrak{B}_{\alpha} \subset \mathscr{K}_{\alpha}^{+}$. Кроме того, $\mathfrak{A}_{\alpha}=\Pi_{+} \mathscr{K}_{\alpha}$, где $\mathscr{K}_{\alpha}-$ ядро системы (2.2)-(2.3). Наконец, в силу утверждения 2.2, траекторные аттракторы $\mathfrak{A}_{\alpha}$ равномерно (по $\left.\alpha \in(0,1]\right)$ ограничены в $\mathscr{F}_{+}^{\mathrm{b}}$. Из теоремы 2.1 получаем следующее утверждение.

СледСтвиЕ 2.3. Имеет место соотношение

$$
\mathfrak{A}_{\alpha} \rightarrow \mathfrak{A}_{0} \quad \text { в топологии } \Theta_{+}^{\text {loc }} \text { при } \alpha \rightarrow 0+.
$$

В самом деле, семейство $\left\{\mathfrak{A}_{\alpha}, 0<\alpha \leqslant 1\right\}$ равномерно ограничено в $\mathscr{F}_{+}^{\mathrm{b}}$.

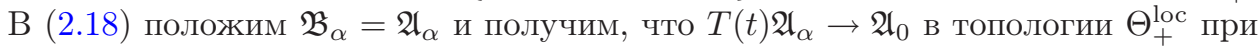
$t \rightarrow+\infty, \alpha \rightarrow 0+$. Однако $T(t) \mathfrak{A}_{\alpha}=\mathfrak{A}_{\alpha}$ при всех $t \geqslant 0$, и, следовательно, $\mathfrak{A}_{\alpha} \rightarrow \mathfrak{A}_{0}$ в топологии $\Theta_{+}^{\text {loc }}$ при $\alpha \rightarrow 0+$.

Кроме того, справедливо следующее утверждение.

СлЕДСТвиЕ 2.4. Для любъх $M>0 u \delta>0$

$$
\begin{aligned}
& \operatorname{dist}_{L_{2}\left(0, M ; H^{1-\delta}\right)}\left(\mathfrak{A}_{\alpha}, \mathfrak{A}_{0}\right) \rightarrow 0+, \\
& \operatorname{dist}_{C\left([0, M] ; H^{-\delta}\right)}\left(\mathfrak{A}_{\alpha}, \mathfrak{A}_{0}\right) \rightarrow 0+
\end{aligned}
$$

при $\alpha \rightarrow 0+$.

В заключение установим связь между траекторным аттрактором $\mathfrak{A}_{\alpha}$ и глобальным аттрактором $\mathscr{A}_{\alpha} \alpha$-модели Лерэ при фиксированном $\alpha>0$ :

$$
\mathfrak{A}_{\alpha}=\left\{v(t)=S(t) v_{0}, t \geqslant 0 \mid v_{0} \in \mathscr{A}_{\alpha}\right\} .
$$

Из оценки (2.13) выводится, что при фиксированном $\alpha>0$ траекторный аттрактор $\mathfrak{A}_{\alpha}$ ограничен в пространстве $\mathscr{F}_{+}^{\mathrm{b}, \mathrm{s}}=L_{2}^{\mathrm{b}}\left(\mathbb{R}_{+} ; H^{2}\right) \cap L_{\infty}\left(\mathbb{R}_{+} ; V\right) \cap$ $\left\{\partial_{t} w(\cdot) \in L_{2}^{\mathrm{b}}\left(\mathbb{R}_{+} ; H\right)\right\}$ и $\mathfrak{A}_{\alpha}$ притягивает ограниченные семейства траекторий из $\mathscr{K}_{\alpha}^{+}$в сильной топологии $\Theta_{+}^{\text {loc,s }}$, которая порождается сильной локальной сходимостью в $L_{2}^{\text {loc }}\left(\mathbb{R}_{+} ; H^{2}\right) \cap L_{\infty}^{\text {loc }}\left(\mathbb{R}_{+} ; V\right) \cap\left\{\partial_{t} w(\cdot) \in L_{2}^{\text {loc }}\left(\mathbb{R}_{+} ; H\right)\right\}$. Однако эти свойства не сохраняю в пределе при $\alpha \rightarrow 0+$.

Для глобальных аттракторов $\mathscr{A}_{\alpha} \alpha$-модели Лерэ возможен предельный переход в слабой топологии пространства $H$ к слабому глобальному аттрактору $\mathscr{A}_{0}$ 3D-системы Навье-Стокса (2.15).

СлеДСтвиЕ 2.5. Глобалъный аттрактор $\mathscr{A}_{\alpha} \alpha$-модели Лерэ сходится при $\alpha \rightarrow 0+\kappa$ слабому глобальному аттрактору $\mathscr{A}_{0}$ 3D-системъ Навъе-Стокca $8 H_{\mathrm{w}}$. 
Сформулируем еще два важных свойства траекторных аттракторов $\mathscr{A}_{\alpha}$, которые выводятся из однозначной разрешимости задачи Коши (2.2), (2.3), (2.9).

УтВЕРЖДЕНИЕ 2.4. При любом $\alpha>0$ траекторный аттрактор $\mathfrak{A}_{\alpha}$ является свлзным множеством в топологическом пространстве $\mathscr{F}_{+}^{\text {loc }} \cap \Theta_{+}^{\text {loc }}$.

УТВЕРЖДЕНИЕ 2.5. Семейство множеств $\left\{\mathfrak{A}_{\alpha}, 0<\alpha \leqslant 1\right\}$ непрерывно сверху в топологии $\Theta_{+}^{\text {loc }}$, т.е. при каждом $\alpha, 0<\alpha \leqslant 1$, и для любой окрестности $\mathscr{O}\left(\mathfrak{A}_{\alpha}\right)$ найдется число $\delta=\delta(\alpha, \mathscr{O})>0$ такое, что

$$
\mathfrak{A}_{\alpha^{\prime}} \subseteq \mathscr{O}\left(\mathfrak{A}_{\alpha}\right), \quad \forall \alpha^{\prime}>0, \quad\left|\alpha^{\prime}-\alpha\right|<\delta .
$$

О минимальном пределе траекторных аттракторов. Выше было установлено, что семейство $\left\{\mathfrak{A}_{\alpha}, 0<\alpha \leqslant 1\right\}$ траекторных аттракторов $\alpha$-модели Лерэ равномерно ограничено в $\mathscr{F}_{+}^{\mathrm{b}}$, т. е. $\mathfrak{A}_{\alpha} \subset \mathscr{B}$ при всех $\alpha \in(0,1]$, где $\mathscr{B}$ - это шар в $\mathscr{F}_{+}^{\mathrm{b}}$ с некоторым радиусом $R$, не зависящим от $\alpha$ :

$$
\left\|\mathfrak{A}_{\alpha}\right\|_{\mathscr{F}_{+}^{\mathrm{b}}} \leqslant\|\mathscr{B}\|_{\mathscr{F}_{+}^{\mathrm{b}}}=R, \quad \forall \alpha, 0<\alpha \leqslant 1 .
$$

Ясно, что траекторный аттрактор $\mathfrak{A}_{0}$ точной системы Навье-Стокса также принадлежит $\mathscr{B}$ (см. раздел 5 главы II). Напомним, что шар $\mathscr{B}$ компактен в топологии $\Theta_{+}^{\text {loc }}$. Из теоремы Урысона о компактности вытекает, что подпространство $\mathscr{B} \cap \Theta_{+}^{\text {loc }}$, снабженное топологией из $\Theta_{+}^{\text {loc }}$, является метризуемым (см. подробности в [12]). Обозначим соответствующую метрику в $\mathscr{B} \cap \Theta_{+}^{\text {loc }}$ через $\rho(\cdot, \cdot)$, а само метрическое пространство обозначим $\mathscr{B} \rho$. Это метрическое пространство компактно и полно. Используя новые обозначения, предельное соотношение (2.19) можно записать в виде

$$
\operatorname{dist}_{\mathscr{B}}\left(\mathfrak{A}_{\alpha}, \mathfrak{A}_{0}\right) \rightarrow 0+,
$$

где $\operatorname{dist}_{\mathscr{B}_{\rho}}(\cdot, \cdot)$ обозначает расстояние Хаусдорфа в метрике пространства $\mathscr{B} \rho$.

Напомним, что множество $\mathfrak{A}_{0} \subset \mathscr{B}_{\rho}$ замкнуто в $\mathscr{B}_{\rho}$. Рассмотрим в $\mathfrak{A}_{0}$ минимальное замкнутое подмножество, которое удовлетворяет предельному соотношению (2.22). Введем следующее определение.

ОПРЕДЕЛЕНИЕ 2.1. Замкнутое подмножество $\mathfrak{A}_{\min } \subseteq \mathfrak{A}_{0}$ называется минималъным пределом траекторных аттракторов $\mathfrak{A}_{\alpha}$ при $\alpha \rightarrow 0+$, если оно удовлетворяет свойству (2.22), т. е. имеет место равенство

$$
\lim _{\alpha \rightarrow 0+} \operatorname{dist}_{\mathscr{B}_{\rho}}\left(\mathfrak{A}_{\alpha}, \mathfrak{A}_{\min }\right)=0,
$$

и, кроме того, $\mathfrak{A}_{\text {min }}$ принадлежит каждому замкнутому подмножеству $\mathfrak{A}^{\prime} \subseteq \mathfrak{A}_{0}$, для которого $\lim _{\alpha \rightarrow 0+} \operatorname{dist}_{\mathscr{B}_{\rho}}\left(\mathfrak{A}_{\alpha}, \mathfrak{A}^{\prime}\right)=0$.

Такое минимальное подмножество существует, и оно строится по формуле

$$
\mathfrak{A}_{\min }=\bigcap_{0<\delta \leqslant 1}\left[\bigcup_{0<\alpha \leqslant \delta} \mathfrak{A}_{\alpha}\right]_{\mathscr{B}_{\rho}}
$$

(см. [90]). 
Теорема 2.2. Минимальный предел $\mathfrak{A}_{\text {min }}$ траекторных аттракторов $\mathfrak{A}_{\alpha}$ при $\alpha \rightarrow 0+$ является связным подмножеством $\mathfrak{A}_{0}$ в $\mathscr{B}_{\rho}$. Множество $\mathfrak{A}_{\text {min }}$ строго инвариантно относительно трансляционной полугруппъ $\{T(h)\}$, m.e.

$$
T(t) \mathfrak{A}_{\min }=\mathfrak{A}_{\min }, \quad \forall t \geqslant 0 .
$$

ДокАЗАТЕЛЬство. Предположим, что множество $\mathfrak{A}_{\min }$ не связно. Тогда $\mathfrak{A}_{\text {min }}$ представимо в виде объединения двух непустых замкнутых непересекающихся подмножеств $\mathfrak{A}_{\text {min }}^{1}$ и $\mathfrak{A}_{\text {min }}^{2}$, т. е.

$$
\mathfrak{A}_{\min }=\mathfrak{A}_{\min }^{1} \cup \mathfrak{A}_{\min }^{2} \quad \text { и } \quad \mathfrak{A}_{\min }^{1} \cap \mathfrak{A}_{\min }^{2}=\varnothing .
$$

Поскольку метрическое пространство $\mathscr{B} \rho$ компактно, имеются два открытых множества $\mathscr{O}_{1}$ и $\mathscr{O}_{2}$ в $\mathscr{B}_{\rho}$ такие, что $\mathfrak{A}_{\text {min }}^{1} \subset \mathscr{O}_{1}, \mathfrak{A}_{\text {min }}^{2} \subset \mathscr{O}_{2}$ и $\mathscr{O}_{1} \cap \mathscr{O}_{2}=\varnothing$. Ясно, что $\mathfrak{A}_{\min } \subset \mathscr{O}_{1} \cup \mathscr{O}_{2}$. Следовательно, в силу (2.22) найдется число $\alpha_{0}>0$ такое, что

$$
\mathfrak{A}_{\alpha} \subset \mathscr{O}_{1} \cup \mathscr{O}_{2}, \quad \forall \alpha, \quad 0<\alpha \leqslant \alpha_{0} .
$$

Заметим, что каждое множество $\mathfrak{A}_{\alpha}$ связно (см. утверждение 2.4 ), т. е. $\mathfrak{A}_{\alpha} \subset \mathscr{O}_{1}$ или $\mathfrak{A}_{\alpha} \subset \mathscr{O}_{2}$ при всех $\alpha<\alpha_{0}$. В то же время, поскольку $\mathfrak{A}_{\min }-$ это минимальнъй предел $\mathfrak{A}_{\alpha}$, можно найти $\alpha_{1}$ и $\alpha_{2}$ такие, что

$$
\mathfrak{A}_{\alpha_{1}} \subset \mathscr{O}_{1} \quad \text { и } \quad \mathfrak{A}_{\alpha_{2}} \subset \mathscr{O}_{2}
$$

(в противном случае можно уменьшить $\mathfrak{A}_{\min }$ ). Пусть, для определенности, $0<\alpha_{2}<\alpha_{1}<\alpha_{0}$. Положим

$$
\delta^{*}=\sup \left\{\delta: \mathfrak{A}_{\alpha} \subset \mathscr{O}_{2}, \alpha_{2} \leqslant \alpha<\alpha_{2}+\delta\right\} .
$$

Заметим, что $\alpha_{2}+\delta^{*} \leqslant \alpha_{1}<\alpha_{0}$ (см. (2.26)) и $\mathfrak{A}_{\alpha_{2}+\delta^{*}} \subset \mathscr{O}_{1} \cup \mathscr{O}_{2}$, поскольку $\alpha_{2}+\delta^{*}<\alpha_{0}$ (см. (2.25)).

Утверждается, что $\mathfrak{A}_{\alpha_{2}+\delta^{*}}$ не может принадлежать $\mathscr{O}_{2}$. В самом деле, если $\mathfrak{A}_{\alpha_{2}+\delta^{*}} \subset \mathscr{O}_{2}$, то, в силу утверждения 2.5 , имеется достаточно малое $\delta_{2}>0$ такое, что $\mathfrak{A}_{\alpha_{2}+\delta^{*}+\delta_{2}} \subset \mathscr{O}_{2}$, а это противоречит определению $\delta^{*}$ в (2.27). Одновременно с этим, $\mathfrak{A}_{\alpha_{2}+\delta^{*}}$ не может принадлежать и $\mathscr{O}_{1}$. Действительно, если $\mathfrak{A}_{\alpha_{2}+\delta^{*}} \subset \mathscr{O}_{1}$, то, опять в силу утверждения 2.5 , найдется число $\delta_{1}>0$ такое, что $\mathfrak{A}_{\alpha_{2}+\delta^{*}-\delta_{1}} \subset \mathscr{O}_{1}$, а это снова противоречит определению $\delta^{*}$. Однако все это вступает в противоречие с включением $\mathfrak{A}_{\alpha_{2}+\delta^{*}} \subset \mathscr{O}_{1} \cup \mathscr{O}_{2}$.

Мы доказали связность множества $\mathfrak{A}_{\text {min. }}$ Установим теперь свойство (2.24).

Рассмотрим произвольную точку $w \in \mathfrak{A}_{\min }$. По определению, найдется последовательность $w_{\alpha_{n}} \in \mathfrak{A}_{\alpha_{n}}$ такая, что $\rho\left(w_{\alpha_{n}}, w\right) \rightarrow 0$ при $\alpha_{n} \rightarrow 0+$. Трансляционная полугруппа $\{T(t)\}$ является непрерывной в $\Theta_{+}^{\text {loc }}$, а значит,

$$
\rho\left(T(t) w_{\alpha_{n}}, T(t) w\right) \rightarrow 0 \quad \text { при } \alpha_{n} \rightarrow 0+.
$$

Раз каждое множество $\mathfrak{A}_{\alpha_{n}}$ строго инвариантно, $T(t) w_{\alpha_{n}} \in \mathfrak{A}_{\alpha_{n}}$. Следовательно, $T(t) w \in \mathfrak{A}_{\min }$, и мы доказали, что

$$
T(t) \mathfrak{A}_{\min } \subseteq \mathfrak{A}_{\min }, \quad \forall h \geqslant 0 .
$$


Проверим обратное включение. Для любого $h \geqslant 0$ и для произвольной точки $w \in \mathfrak{A}_{\text {min }}$ с соответствующей последовательностью $w_{\alpha_{n}} \in \mathfrak{A}_{\alpha_{n}}, \rho\left(w_{\alpha_{n}}, w\right) \rightarrow 0$ $\left(\alpha_{n} \rightarrow 0+\right)$, необходимо найти точку $W \in \mathfrak{A}_{\min }$ такую, что $T(t) W=w$. Поскольку множество $\mathfrak{A}_{\alpha_{n}}$ строго инвариантно, то найдутся элементы $W_{\alpha_{n}} \in \mathfrak{A}_{\alpha_{n}}$ такие, что $T(h) W_{\alpha_{n}}=w_{\alpha_{n}}$. Последовательность $\left\{W_{\alpha_{n}}\right\}$ принадлежит компактному множеству $\mathscr{B}_{\rho}$. Переходя к подпоследовательности $\left\{\alpha_{n^{\prime}}\right\}$, имеем, что $W_{\alpha_{n^{\prime}}} \rightarrow W\left(n^{\prime} \rightarrow \infty\right)$ для некоторого $W \in \mathscr{B}_{\rho}$. Тогда $W \in \mathfrak{A}_{\min }$. Поскольку $\{T(t)\}$ непрерывно, то $T(t) W_{\alpha_{n^{\prime}}} \rightarrow T(t) W\left(n^{\prime} \rightarrow \infty\right)$. Однако $T(t) W_{\alpha_{n^{\prime}}}=w_{\alpha_{n^{\prime}}}$, и тогда $w_{\alpha_{n^{\prime}}} \rightarrow T(t) W\left(n^{\prime} \rightarrow \infty\right)$, но $w_{\alpha_{n}} \rightarrow w(n \rightarrow \infty)$. Следовательно, $T(t) W=w$, и доказано включение

$$
\mathfrak{A}_{\text {min }} \subseteq T(t) \mathfrak{A}_{\min }, \quad \forall t \geqslant 0 .
$$

В итоге получаем (2.24). Теорема доказана.

\section{3. Предел нулевой вязкости траекторного аттрактора диссипативной 2D-системы Навье-Стокса}

\section{Траекторный аттрактор диссипативной 2D-системы Навье-Стокса.} Рассматривается диссипативная двумерная система Навье-Стокса:

$$
\partial_{t} u+B(u, u)-\nu \Delta u+r u=g(x), \quad(\nabla, u)=0, \quad x=\left(x_{1}, x_{2}\right) \in \mathbb{T}^{2}, \quad t \geqslant 0,
$$

которая содержит дополнительное слагаемое $-r u$. Мы используем те же обозначения, что и в разделе 3 главы II при изучении диссипативной 2D-системы Эйлера (II.3.1). В уравнениях (3.1) $\nu>0$ обозначает кинематическую вязкость. Давление $p(x, t)$ устранено с помощью применения к обеим частям оператора Лерэ $P$.

Система (3.1) встречается в ряде проблем геофизики (см. [58]).

Начальные условия задаются при $t=0$ :

$$
\left.u\right|_{t=0}=u_{0}(x), \quad u_{0}(x) \in H^{1} .
$$

Задача Коши (3.1), (3.2) однозначно разрешима. При этом, если $u_{0} \in H^{1}$, то соответствующее решение $u(x, t)$ принадлежит $C_{\mathrm{b}}\left(\mathbb{R}_{+} ; H^{1}\right) \cap L_{2}^{\mathrm{b}}\left(\mathbb{R}_{+} ; H^{2}\right)$, а ее производная по времени $\partial_{t} u$ принадлежит $L_{2}^{\mathrm{b}}\left(\mathbb{R}_{+} ; H\right)$. Такое решение иногда называют сильным решением. Случай $r=0$, отвечающей классической 2D-системе Навье-Стокса, хорошо изучен (см. [1], [2], [11], [12], [25], [45]). Добавочный диссипативный член $-r u$ влияет не принципиально на эти результаты (см. [91], [92]). Любое решение $u(x, t)$ задачи (3.1), (3.2) удовлетворяет следующим тождествам:

$$
\begin{aligned}
\frac{1}{2} \frac{d}{d t}|u(t)|^{2}+\nu|\nabla u(t)|^{2}+r|u(t)|^{2} & =(g, u(t)), \\
\frac{1}{2} \frac{d}{d t}|\nabla u(t)|^{2}+\nu|\Delta u(t)|^{2}+r|\nabla u(t)|^{2} & =(\nabla g, \nabla u(t)),
\end{aligned}
$$

которые аналогичны соответствующим тождествам при $r=0$, доказанным в разделе 3 главы II. Из этих соотношений следует неравенство

$$
\|u(t)\|^{2} \leqslant\left\|u_{0}\right\|^{2} \mathrm{e}^{-r t}+r^{-1}\|g\|^{2},
$$

которое подобно (II.3.13). Напомним, что $\|w\|^{2}=|\nabla w|^{2}+|w|^{2}=\|w\|_{H^{1}}^{2}$. 
С помощью интегрирования из (3.3) и (3.4) выводим неравенство

$$
2 \nu \int_{t}^{t+1}|\Delta u(s)|^{2} d s \leqslant\left\|u_{0}\right\|^{2} \mathrm{e}^{-r t}+2 r^{-1}\|g\|^{2} .
$$

Из уравнения (3.1) следует, что

$$
\left\|\partial_{t} u(t)\right\|_{H^{-1}} \leqslant C_{1}\left(\|u(t)\|^{2}+\|g\|^{2}+1\right),
$$

где константа $C_{1}$ такая же, как в неравенстве (II.3.17), и не зависит от $\nu$. Поэтому, в силу (3.5), находим, что

$$
\left\|\partial_{t} u(t)\right\|_{H^{-1}} \leqslant C_{1}\left(\left\|u_{0}\right\|^{2} \mathrm{e}^{-r t}+\left(1+r^{-1}\right)\|g\|^{2}+1\right) .
$$

Приближения к траекторному аттрактору диссипативной 2D-системы Эйлера. Рассмотрим теперь вопрос о сходимости траекторных аттракторов $\mathfrak{A}_{\nu}$ системы $(3.1)$ при $\nu \rightarrow 0+$ к траекторному аттрактору $\mathfrak{A}$ диссипативной 2D-системы Эйлера (3.1).

Аналогично разделу 3 главы II рассматривается пространство траекторий системы (3.1)

$$
\mathscr{K}_{\nu}^{+} \subset C_{\mathrm{b}}\left(\mathbb{R}_{+} ; H^{1}\right) \cap L_{2}^{\mathrm{b}}\left(\mathbb{R}_{+} ; H^{2}\right) \subset \mathscr{F}_{+}^{\infty},
$$

состоящее из всех ее сильных решений при фиксированной вязкости $\nu>0$. По определению, множеству $\mathscr{K}_{\nu}^{+}$принадлежат все решения $u(x, s), s \geqslant 0$, этой системы с любыми начальными данными $u_{0}=u(0) \in H^{1}$. Пространства $\mathscr{F}_{+}^{\infty} \subset \mathscr{F}_{+}^{\text {loc }}$ и топология $\Theta_{+}^{\text {loc }}$ были определены в разделе 3 главы II. Доказано, что пространство траекторий $\mathscr{K}_{\nu}^{+}$замкнуто в топологии $\Theta_{+}^{\text {loc }}$ (см. [62]).

Трансляционная полугруппа $\{T(t)\}$ действует на пространстве $\mathscr{K}_{\nu}^{+}$по формуле $T(t) u(s)=u(t+s)$. Ясно, что $T(t) \mathscr{K}_{\nu}^{+} \subseteq \mathscr{K}_{\nu}^{+}$при всех $t \geqslant 0$. Аналогично разделу 3 главы II доказывается, что полугруппа $\{T(t)\}$ на $\mathscr{K}_{\nu}^{+}$имеет траекторный аттрактор $\mathfrak{A}_{\nu} \subset \mathscr{K}_{\nu}^{+}$, который притягивает ограниченные (в $\left.\mathscr{F}_{+}^{\infty}\right)$ множества траекторий системы (3.1) в топологии $\Theta_{+}^{\text {loc }}$.

Из неравенств $(3.5),(3.6)$ и (3.8) следует, что траекторные аттракторы $\mathfrak{A}_{\nu}$ равномерно (по $0<\nu \leqslant 1$ ) ограничены в $\mathscr{F}_{+}^{\infty}$, т. е. для любого $u_{\nu} \in \mathfrak{A}_{\nu}$

$$
\begin{aligned}
\left\|u_{\nu}(\cdot)\right\|_{L_{\infty}\left(\mathbb{R}_{+} ; H^{1}\right)} & \leqslant r^{-1}\|g\|^{2} \\
\left\|\partial_{t} u_{\nu}(\cdot)\right\|_{L_{\infty}\left(\mathbb{R}_{+} ; H^{-1}\right)} & \leqslant C_{1}\left(\left(1+r^{-1}\right)\|g\|^{2}+1\right) .
\end{aligned}
$$

Сравнивая неравенства (II.3.31), (II.3.32) с неравенствами (3.10), (3.11), замечаем, что траекторный аттрактор $\mathfrak{A}$ диссипативной системы Эйлера (II.3.1) и траекторные аттракторы $\mathfrak{A}_{\nu}$ системы (3.1) принадлежат одному и тому же шару $\mathscr{B}\left(0, R_{0}\right)$ в $\mathscr{F}_{+}^{\infty}$ с радиусом $R_{0}, R_{0}^{2}=\max \left\{r^{-1}\|g\|^{2}, C_{1}\left(\left(1+r^{-1}\right)\|g\|^{2}+1\right)\right\}$ :

$$
\|\mathfrak{A}\|_{\mathscr{F}_{+}^{\mathrm{b}}} \leqslant R_{0} \quad \text { и } \quad\left\|\mathfrak{A}_{\nu}\right\|_{\mathscr{F}_{+}^{\mathrm{b}}} \leqslant R_{0}, \quad \forall \nu, \quad 0<\nu \leqslant 1 .
$$

Далее через $\rho$ обозначается метрика на этом шаре, порождающая $\Theta_{+}^{\text {loc }}$.

Сформулируем основной результат этого раздела. 
ТеОрема 3.1. Траекторные аттракторы $\mathfrak{A}_{\nu}$ системы (3.1) сходятся при $\nu \rightarrow 0+$ в метрике $\rho \kappa$ траекторному аттрактору $\mathfrak{A}$ системы (II.3.1):

$$
\operatorname{dist}_{\rho}\left(\mathfrak{A}_{\nu}, \mathfrak{A}\right) \rightarrow 0+\quad \text { npu } \nu \rightarrow 0+.
$$

Подробное доказательство этой теоремы приведено в [62].

Заметим, что траектории, лежащие на аттракторе $\mathfrak{A}_{\nu}$, являются более гладкими функциями, чем те, что лежат на $\mathfrak{A}$, так как в силу (3.6)

$$
\nu \int_{h}^{h+1}\left|\Delta u_{\nu}(s)\right|^{2} d s \leqslant r^{-1}\|g\|^{2}, \quad h \geqslant 0, \quad \forall u_{\nu} \in \mathfrak{A}_{\nu} .
$$

Изучим теперь минимальный предел $\mathfrak{A}_{\text {min }}$ траекторных аттракторов $\mathfrak{A}_{\nu}$ при $\nu \rightarrow 0+$ аналогично тому, как в разделе 2 мы исследовали минимальный предел траекторных аттракторов $\alpha$-модели Лерэ.

Как уже отмечалось, множества $\mathfrak{A}$ и $\mathfrak{A}_{\nu}$ принадлежат шару $\mathscr{B}_{0}=\mathscr{B}\left(0, R_{0}\right)$ в $\mathscr{F}_{+}^{\mathrm{b}}$ с радиусом $R_{0}$, не зависящим от $\nu,($ см. $(3.12))$ :

$$
\mathfrak{A} \subset \mathscr{B}_{0} \quad \text { и } \quad \mathfrak{A}_{\nu} \subset \mathscr{B}_{0}, \quad \forall \nu \in(0,1]
$$

причем $\mathscr{B}_{0}$ является компактным (в топологии $\Theta_{+}^{\text {loc }}$ ) метрическим пространством с метрикой $\rho(\cdot, \cdot)$. Напомним, что множество $\mathfrak{A} \subset \mathscr{B}_{0}$ замкнуто в пространстве $\mathscr{B}_{0}$ и удовлетворяет свойству притяжения (3.13).

Пусть $\mathfrak{A}_{\text {min }}$ является минимальным замкнутым подмножеством $\mathfrak{A}$, которое удовлетворяет свойству (3.15), т. е.

$$
\lim _{\nu \rightarrow 0+} \operatorname{dist}_{\rho}\left(\mathfrak{A}_{\nu}, \mathfrak{A}_{\min }\right)=0
$$

и $\mathfrak{A}_{\text {min }}$ принадлежит каждому замкнутому подмножеству $\mathfrak{A}^{\prime} \subseteq \mathfrak{A}$, для которого

$$
\lim _{\nu \rightarrow 0+} \operatorname{dist}_{\rho}\left(\mathfrak{A}_{\nu}, \mathfrak{A}^{\prime}\right)=0 .
$$

Мы будем называть такое множество $\mathfrak{A}_{\text {min }}$ минималъным пределом траекторных аттракторов $\mathfrak{A}_{\nu}$ при $\nu \rightarrow 0+$.

Утверждается, что множество $\mathfrak{A}_{\min }$ существует и единственно. Его можно построить по формуле

$$
\mathfrak{A}_{\min }=\bigcap_{0<\delta \leqslant 1}\left[\bigcup_{0<\nu \leqslant \delta} \mathfrak{A}_{\nu}\right]_{\mathscr{B}_{0}} .
$$

Сформулируем важные свойства $\mathfrak{A}_{\text {min }}$ в виде теоремы, доказательство которой приведено в [62].

Теорема 3.2. Минимальный предел $\mathfrak{A}_{\min } \subseteq \mathfrak{A}$ является связным подмножеством в пространстве $\mathscr{B}_{0}$. Множество $\mathfrak{A}_{\min }$ строго инвариантно относительно полугруппи $\{T(t)\}$. 


\section{4. Система реакции-диффузии с малым коэффициентом диффузии}

4.1. Слабые решения системы реакции-диффузии. В ограниченной области $\Omega \Subset \mathbb{R}^{3}$ с гладкой границей $\partial \Omega$ рассматривается система уравнений с малым параметром $\delta>0$ :

$$
\begin{array}{ll}
\partial_{t} u=\Delta u-f(u, v)+g_{1}(x), & \left.u\right|_{\partial \Omega}=0, \\
\partial_{t} v=\delta \Delta v-h(u, v)+g_{2}(x), & \left.v\right|_{\partial \Omega}=0,
\end{array}
$$

относительно неизвестных функций $u=u(x, t)$ и $v=v(x, t), x \in \Omega, t \geqslant 0$. Предполагается, что нелинейные функции $f$ и $h$ являются непрерывными, $f, h: \mathbb{R}^{2} \rightarrow \mathbb{R}$, причем выполнены следующие условия роста по $u$ и $v:$

$$
\begin{gathered}
\sigma_{1}\left(|u|^{p_{1}}+|v|^{p_{2}}\right)-c \leqslant f(u, v) u+h(u, v) v \leqslant C|u|^{p_{1}}+|v|^{p_{2}}+1, \\
|f(u, v)|^{q_{1}}+|h(u, v)|^{q_{2}} \leqslant|u|^{p_{1}}+|v|^{p_{2}}+1, \quad \forall u, v \in \mathbb{R},
\end{gathered}
$$

где $\sigma_{1}, c, C$ - некоторые положительные константы, $p_{1}, p_{2} \geqslant 2$ и $q_{i}=p_{i} /\left(p_{i}-1\right)$, $i=1,2$. Предполагается также, что $h \in C^{1}\left(\mathbb{R}^{2}\right), h(0,0)=0$ и выполнены неравенства

$$
0<\sigma_{2} \leqslant h_{v}(u, v), \quad\left|h_{u}(u, v)\right| \leqslant D, \quad \forall u, v \in \mathbb{R} .
$$

Положительные числа $\sigma_{1}$ и $\sigma_{2}$ в неравенствах (4.3) и (4.5) отражают свойство диссипативности этой системы реакции-диффузии. Обозначим $\sigma=\min \left\{\sigma_{1}, \sigma_{2}\right\}$.

Система (4.1), (4.2) является частным случаем общей системы реакции-диффузии, рассмотренной в разделе 2 главы II. Условия (4.3), (4.4) аналогичны общим условиям (2.2), (2.3), а условие (4.5) отражает специфику данной задачи.

Относительно функций $g_{1}(x)$ и $g_{2}(x)$ в системе (4.1)-(4.2) предполагается, что

$$
g_{1} \in L_{2}(\Omega), \quad g_{2} \in H_{0}^{1}(\Omega) .
$$

Вводятся обозначения: $H:=L_{2}(\Omega), V:=H_{0}^{1}(\Omega)$ и $\|u\|:=\|u\|_{H},\|u\|_{1}:=\|u\|_{V}$.

Примером уравнений (4.1), (4.2) может служить система ФитцХью-Нагумо

$$
\left\{\begin{array}{l}
\partial_{t} u=\Delta u-u(u-\beta)(u-1)-v, \\
\partial_{t} v=\delta \Delta v+\alpha u-\gamma v
\end{array}\right.
$$

в которой $f(u, v)=u(u-\beta)(u-1)+v, h(u, v)=\gamma v-\alpha u$ и $\alpha, \beta, \gamma \geqslant 0$. Для этого примера $\sigma=\min \{1, \gamma\}, p_{1}=4, p_{2}=2$ и $g_{1} \equiv 0, g_{2} \equiv 0$ (см. [2], [93], [94]).

Напомним, что пара функций $(u(x, t), v(x, t))$ называется слабым решением системы (4.1)-(4.2) в области $\Omega \times \mathbb{R}_{+}$, если при любом $M>0$

$$
u \in L_{p_{1}}\left(0, M ; L_{p_{1}}(\Omega)\right) \cap L_{2}(0, M ; V), \quad v \in L_{p_{2}}\left(0, M ; L_{p_{2}}(\Omega)\right) \cap L_{2}(0, M ; V),
$$

причем $u(x, t)$ и $v(x, t)$ удовлетворяют уравнениям $(4.1),(4.2)$ в смысле теории обобщенных функций в $\mathscr{D}^{\prime}\left(0, M ; H^{-r_{1}}(\Omega) \times H^{-r_{2}}(\Omega)\right)$, где обозначено $r_{i}=$ $\max \left\{1,3\left(1 / 2-1 / p_{i}\right)\right\}, i=1,2$ (см. раздел 2 главы II, а также [1], [12], [20]). 
Если $(u(\cdot), v(\cdot))$ - слабое решение $(4.1),(4.2)$, то из этих уравнений выводится, что $u(\cdot) \in L_{\infty}(0, M ; H), v(\cdot) \in L_{\infty}(0, M ; H)$. Кроме того, согласно лемме Лионса-Мадженеса (см. лемму 2.1 в главе II),

$$
u(\cdot) \in C_{\mathrm{w}}([0, M] ; H), \quad v(\cdot) \in C_{\mathrm{w}}([0, M] ; H), \quad \forall M>0 .
$$

Следовательно, при любом $t \geqslant 0$ значения функций $u(x, t)$ и $v(x, t)$ однозначно определены как элементы пространства $H$, и, в частности, корректны начальные условия

$$
\left.u\right|_{t=0}=u_{0},\left.\quad v\right|_{t=0}=v_{0} .
$$

Существование слабого решения системы (4.1)-(4.2) при начальных условиях $u_{0} \in H, v_{0} \in H$ устанавливается с помощью метода Галёркина (см., например, [1], [12]). Доказано, что для любого слабого решения $(u(\cdot), v(\cdot))$ задачи $(4.1),(4.2),(4.7)$ функции $u(\cdot)$ и $v(\cdot)$ принадлежат $C\left(\mathbb{R}_{+} ; H\right)$ и имеют место неравенства

$$
\begin{aligned}
& \|u(t)\|^{2}+\|v(t)\|^{2}+2 \int_{0}^{t}\left(\|u(s)\|_{1}^{2}+\delta\|v(s)\|_{1}^{2}\right) \mathrm{e}^{-\sigma(t-s)} d s \\
& \leqslant\left(\left\|u_{0}\right\|^{2}+\left\|v_{0}\right\|^{2}\right) \mathrm{e}^{-\sigma t}+R_{1}^{2}, \quad \forall t \geqslant 0, \\
& 2 \int_{t}^{t+1}\left(\|u(s)\|_{1}^{2}+\delta\|v(s)\|_{1}^{2}\right) d s+\sigma \int_{t}^{t+1}\left(\|u(s)\|_{L_{p_{1}}}^{p_{1}}+\|v(s)\|_{L_{p_{2}}}^{p_{2}}\right) d s \\
& \leqslant\left(\left\|u_{0}\right\|^{2}+\left\|v_{0}\right\|^{2}\right) \mathrm{e}^{-\sigma t}+R_{2}^{2}, \quad \forall t \geqslant 0,
\end{aligned}
$$

причем величины $R_{1}$ и $R_{2}$ не зависят от $\delta$ (см. раздел 2 главы II).

В том случае, когда $v_{0} \in V$ для слабых решений, которые получаются по методу Галёркина, доказана дополнительная оценка следующего вида:

$$
\|v(t)\|_{1}^{2} \leqslant\left\|v_{0}\right\|_{1}^{2} \mathrm{e}^{-\sigma t}+C\left(\left\|u_{0}\right\|^{2}+\left\|v_{0}\right\|^{2}\right) \mathrm{e}^{-\sigma t}+R^{2}, \quad \forall t \geqslant 0,
$$

где величина $R$ и константа $C$ также не зависят от $\delta$.

Из леммы Лионса-Мадженеса следует, что $v(\cdot) \in C_{\mathrm{w}}\left(\mathbb{R}_{+} ; V\right)$, т. е. значения $v(t) \in V$ однозначно определены при всех $t \geqslant 0$.

Переменная $x$ будет для краткости опускаться. Кроме того, переменную времени в слабых решениях будем обозначать $t=s$, например, $(u(s), v(s))$, $s \geqslant 0$.

Отметим, что при выполнении условий (4.3)-(4.6) задача Коши для системы (4.1)-(4.2) может иметь неединственное решение.

Пространство траекторий и траекторный аттрактор системы реакции-диффузии. Рассматривается пространство $\mathscr{F}_{+}^{\text {loc }}$, состоящее из функций $(y(s), z(s)):=(y(x, s), z(x, s)), x \in \Omega, s \geqslant 0$, где

$$
\begin{aligned}
y(\cdot) & \in L_{\infty}(0, M ; H) \cap L_{2}(0, M ; V) \cap L_{p_{1}}\left(0, M ; L_{p_{1}}(\Omega)\right), \\
z(\cdot) & \in L_{\infty}(0, M ; V) \cap L_{p_{2}}\left(0, M ; L_{p_{2}}(\Omega)\right), \\
\partial_{t} y(\cdot) & \in L_{q_{1}}\left(0, M ; H^{-r_{1}}(\Omega)\right), \\
\partial_{t} z(\cdot) & \in L_{q_{2}}\left(0, M ; H^{-r_{2}}(\Omega)\right)
\end{aligned}
$$


для любого $M>0$. В пространстве таких функций, по общей схеме, изложенной в разделе 4 главы II, строится траекторный аттрактор для системы (4.1)-(4.2).

Как обычно, в пространстве $\mathscr{F}_{+}^{\text {loc }}$ вводится также (слабая) топология $\Theta_{+}^{\text {loc }}:=$ $\Theta_{+}^{\mathrm{w}, \text { loc }}$ с помощью задания слабой или $*$-слабой сходимости последовательностей

$$
\left\{y_{m}(s)\right\}, \quad\left\{z_{m}(s)\right\}, \quad\left\{\partial_{t} y_{m}(s)\right\}, \quad\left\{\partial_{t} z_{m}(s)\right\} \quad \text { при } m \rightarrow \infty
$$

в указанных выше пространствах при каждом $M>0$. Пространство $\mathscr{F}+$ loc с топологией $\Theta_{+}^{\text {loc } я в л я е т с я ~ л и н е и ̆ н ы м ~ х а у с д о р ф о в ы м ~ п р о с т р а н с т в о м ~ Ф р е ш е-У р ы-~}$ сона со счетной базой. В $\mathscr{F}_{+}^{\text {loc }}$ рассматривается линейное подпространство $\mathscr{F}_{+}^{\mathrm{b}}$, состоящее из элементов $(y(\cdot), z(\cdot))$, обладающих конечной нормой

$$
\begin{gathered}
\left.\|(y, z)\|_{\mathscr{F}_{+}^{\mathrm{b}}}:=\|y\|_{L_{\infty}\left(\mathbb{R}_{+} ; H\right)}+\|y\|_{L_{2}^{\mathrm{b}}\left(\mathbb{R}_{+} ; V\right)}\|y\|_{L_{p_{1}}^{\mathrm{b}}\left(\mathbb{R}_{+} ; L_{p_{1}}\right)}+\left\|\partial_{t} y\right\|_{L_{q_{1}}^{\mathrm{b}}\left(\mathbb{R}_{+} ; H^{-r_{1}}\right)}\right) \\
+\|z\|_{L_{\infty}\left(\mathbb{R}_{+} ; V\right)}+\|z\|_{L_{p_{2}}^{\mathrm{b}}\left(\mathbb{R}_{+} ; L_{p_{2}}\right)}+\left\|\partial_{t} z\right\|_{L_{q_{2}}^{\mathrm{b}}\left(\mathbb{R}_{+} ; H^{-r_{2}}\right)} .
\end{gathered}
$$

Отметим, что любой шар $B_{r}=\left\{\|(y, z)\|_{\mathscr{F}_{+}^{\mathrm{b}}} \leqslant r\right\}$ в банаховом пространстве $\mathscr{F}_{+}^{\mathrm{b}}$ компактен в топологии $\mathscr{F}_{+}^{\text {loc }} \cap \Theta_{+}^{\text {loc }}$.

Определим теперь пространство траекторий $\mathscr{K}^{+}(N)$ системы (4.1)-(4.2), которое зависит от числа $N>0$.

ОПРЕДЕЛЕНИЕ 4.1. Пространство $\mathscr{K}^{+}(N)$ состоит из функций $(u(\cdot), v(\cdot)) \in$ $\mathscr{F}_{+}^{\text {loc }}$, которые являются слабыми решениями системы $(4.1)-(4.2)$, причем функция $v(\cdot)$ удовлетворят неравенству

$$
\|v(s)\|_{1}^{2} \leqslant N \mathrm{e}^{-\sigma s}+R^{2}, \quad \forall s \geqslant 0,
$$

где величины $\sigma$ и $R$ такие же, как в неравенстве (4.10).

Из построения слабых решений задачи (4.1), (4.2), (4.7) по методу Галёркина следует, что пространство траекторий $\mathscr{K}^{+}(N)$ не пусто при любом $N>0$.

Рассмотрим трансляционную полугруппу $\{T(t)\}:=\{T(t), t \geqslant 0\}$, действующую в пространстве $\mathscr{F}_{+}^{\text {loc }}$ по формуле $T(t)(y(s), z(s))=(y(t+s), z(t+s))$, $s \geqslant 0$. Отметим, что $T(t): \mathscr{K}^{+}(N) \rightarrow \mathscr{K}^{+}(N)$ при любом $t \geqslant 0$.

УтвеРЖДЕНИЕ 4.1. Пространство траекторий $\mathscr{K}^{+}(N)=\{(u(s), v(s))$, $s \geqslant 0\}$ принадлежит $\mathscr{F}_{+}^{\mathrm{b}}$, причем справедливо неравенство

$$
\|T(t)(u, v)\|_{\mathscr{F}_{+}^{\mathrm{b}}} \leqslant C\left(\|u(0)\|^{2}+N\right) \mathrm{e}^{-\rho t}+R_{3}^{2}, \quad \forall t \geqslant 0,
$$

где $C \geqslant 0$ и $\rho>0$ не зависят от $g_{1}, g_{2} u \delta$, а величина $R_{3}=R_{3}\left(\left\|g_{1}\right\|,\left\|g_{2}\right\|\right)$ не зависит от $\delta$.

УТВЕРЖДЕНИЕ 4.2. Пространство траекторий $\mathscr{K}^{+}(N)$ замкнуто в топологии $\mathscr{F}_{+}^{\text {loc }} \cap \Theta_{+}^{\text {loc }}$ при любом $N \geqslant 0$.

Эти утверждения доказаны в [95].

Построим траекторный аттрактор полугруппы $\{T(t)\}$. Из неравенства (4.12) следует, что множество

$$
P_{0}=\left\{(u, v) \in \mathscr{K}^{+}(N) \mid\|(u, v)\|_{\mathscr{F}_{+}^{\mathrm{b}}} \leqslant 2 R_{3}^{2}\right\}
$$


является поглощающим для полугруппы $\{T(t)\}$ в пространстве $\mathscr{K}^{+}(N)$. Множество $P_{0}$ является ограниченным в $\mathscr{F}_{+}^{\mathrm{b}}$. Рассмотрим на $P_{0}$ топологию, индуцированную из $\Theta_{+}^{\text {loc }}$. Это топологическое пространство является компактным и метризуемым. Легко проверить, что $T(t) P_{0} \subseteq P_{0}$ при $t \geqslant 0$ и полугруппа $\{T(t)\}$ непрерывна на $\mathscr{K}^{+}(N)$ в топологии $\Theta_{+}^{\text {loc }}$. Применима общая теорема о существовании у полугруппы $\{T(t)\}$ глобального аттрактора $\mathfrak{A}(N) \subseteq P_{0}$, который задается формулой

$$
\mathfrak{A}(N)=\bigcap_{t \geqslant 0} T(t) P_{0} .
$$

Множество $\mathfrak{A}(N)$ ограничено в $\mathscr{F}_{+}^{\mathrm{b}}$, компактно в $\Theta_{+}^{\text {loc }}$, строго инвариантно и, очевидно, притягивает любое ограниченное множество $B \subset \mathscr{K}^{+}(N)$. Следовательно, построенное по формуле (4.14) множество $\mathfrak{A}(N)$ является траекторным аттрактором $\{T(\tau)\}$ в $\mathscr{K}^{+}(N)$. В [95] доказано следующее утверждение.

УтВЕРЖДЕНИЕ 4.3. Построенный в пространстве $\mathscr{K}^{+}(N)$ траекторный аттрактор не зависит от числа $N: \mathfrak{A}(N)=\mathfrak{A}$, в частности, $\mathfrak{A}=\mathfrak{A}(0)$, т.е.

$$
\sup \left\{\|v(t)\|_{1}^{2} \mid t \geqslant 0\right\} \leqslant R^{2}, \quad \forall(u, v) \in \mathfrak{A},
$$

где $R$ такое же, как в (4.10).

Далее описывается структура траекторного аттрактора $\mathfrak{A}$ с помощью полных траекторий $\{u(s), v(s)\}, s \in \mathbb{R}$, системы уравнений (4.1)-(4.2). Вводятся пространства $\mathscr{F}^{\mathrm{loc}}, \mathscr{F}^{\mathrm{b}}$ и $\Theta^{\mathrm{loc}}$, которые определяются аналогично $\mathscr{F}_{+}^{\mathrm{loc}}, \mathscr{F}_{+}^{\mathrm{b}}$ и $\Theta_{+}^{\text {loc }}$ с заменой в их определениях полупрямой $\mathbb{R}_{+}(s \geqslant 0)$ на всю прямую $\mathbb{R}$ $(-\infty<s<\infty)$. Рассматриваются слабые решения $\{u(s), v(s)\}, s \in \mathbb{R}$, системы (4.1)-(4.2), которые принадлежат пространству $\mathscr{F}$.

ОПредЕЛЕНИЕ 4.2. Ядром $\mathscr{K}$ системы уравнений (4.1)-(4.2) в пространстве $\mathscr{F}^{\mathrm{b}}$ называется множество всех его слабых решений $\{u(s), v(s)\}, s \in \mathbb{R}$, которые принадлежат $\mathscr{F}^{\mathrm{b}}$ и для которых выполнено неравенство

$$
\sup \left\{\|v(t)\|_{1} \mid t \in \mathbb{R}\right\} \leqslant R,
$$

где величина $R$ такая же, как в (4.10).

Tеорема 4.1. Ядро $\mathscr{K}$ системы (4.1)-(4.2) ограничено в пространстве $\mathscr{F} \mathrm{b}$ и компактно в топологии $\Theta^{\text {loc }}$, причем $\mathfrak{A}=\Pi_{+} \mathscr{K}$, где $\Pi_{+}-$оператор ограничения функииц $\{f(s), s \in \mathbb{R}\}$ на полуосъ $\mathbb{R}_{+}$.

В дальнейшем используются обозначения $\mathfrak{A}_{\delta}:=\mathfrak{A}$ и $\mathscr{K}_{\delta}:=\mathscr{K}$, где $\delta$ - малый коэффициент диффузии в уравнении (4.2). Напомним, что $\mathfrak{A}_{\delta} \subset P_{0}$ при всех $\delta \in(0,1]$ (см. (4.13)), причем множество $P_{0}$ не зависит от $\delta$.

СлеДСтвие 4.1. Семейство траекторных аттракторов $\left\{\mathfrak{A}_{\delta}, 0<\delta \leqslant 1\right\}$ равномерно ограничено по норме $\mathscr{F}_{+}^{\mathrm{b}}$, а семейство ядер $\left\{\mathscr{K}_{\delta}, 0<\delta \leqslant 1\right\}$ равномерно ограничено в пространстве $\mathscr{F} \mathrm{b}$. 
Предельная система реакции-диффузии и ее траекторный аттрактор. Рассматривается предельная относительно (4.1)-(4.2) система уравнений

$$
\begin{array}{ll}
\partial_{t} u=\Delta u-f(u, v)+g_{1}(x), & \left.u\right|_{\partial \Omega}=0, \\
\partial_{t} v=-h(u, v)+g_{2}(x), & \left.v\right|_{\partial \Omega}=0 .
\end{array}
$$

В этом случае уравнение (4.16) фактически становится обыкновенным дифференциальным уравнением относительно неизвестной функции $v(x, t), t \geqslant 0$, при каждом фиксированном $x \in \Omega$. Такие частично диссипативные системы реакции-диффузии рассматривались, например, в [96], где накладывались дополнительные ограничения на нелинейные члены уравнений, которые гарантируют однозначную разрешимость начальной задачи.

Предполагается, что функции $f$ и $h$ в системе (4.15)-(4.16) удовлетворяют неравенствам (4.3)-(4.5), а для функций $g_{1}$ и $g_{2}$ выполнены включения (4.6). Легко установить, что эти условия не обеспечивают однозначную разрешимость соответствующей задачи Коши.

Слабым решением системы (4.15)-(4.16) в области $\Omega \times \mathbb{R}_{+}$называется пара функций $(u(x, t), v(x, t))$, принадлежащих пространствам

$$
L_{p_{1}}\left(0, M ; L_{p_{1}}(\Omega)\right) \cap L_{2}(0, M ; V) \quad \text { и } \quad L_{p_{2}}\left(0, M ; L_{p_{2}}(\Omega)\right) \cap L_{\infty}(0, M ; V)
$$

соответственно и удовлетворяющих уравнениям (4.15), (4.16) в пространстве обобщенных функций $\mathscr{D}^{\prime}\left(0, M ; H^{-r_{1}}(\Omega) \times H^{-r_{2}}(\Omega)\right)$ при каждом $M>0$ (показатели $r_{1}$ и $r_{2}$ были определены в разделе 1$)$. При $t=0$ задаются начальные условия

$$
\left.u\right|_{t=0}=u_{0} \in H,\left.\quad v\right|_{t=0}=v_{0} \in V .
$$

Существование слабых решений задачи (4.15)-(4.17) доказывается с помощью метода Галёркина, который был описан в разделе 1. Для любого слабого решения имеют место оценки (4.8) и (4.9), в которых $\delta=0$. Кроме того, доказано существование слабого решения $(u(x, t), v(x, t))$ с любыми начальными условиями (4.17), которое удовлетворяет (4.10).

Для построения траекторного аттрактора системы (4.15)-(4.16) вводится пространство $\mathscr{K}_{0}^{+}(N)$ аналогично пространству $\mathscr{K}_{\delta}^{+}(N):=\mathscr{K}^{+}(N)$ системы (4.1)-(4.2). Пространство $\mathscr{K}_{0}^{+}(N)$ состоит из функций $(u(\cdot), v(\cdot)) \in \mathscr{F}_{+}^{\text {loc }}$, которые являются слабыми решениями $(4.15),(4.16)$, причем функция $v(\cdot)$ удовлетворят неравенству (4.11) с теми же константами $\sigma$ и $R$.

Как и выше, доказывается, что трансляционная полугруппа $\{T(t)\}$, действующая в пространстве $\mathscr{K}_{0}^{+}(N)$, имеет траекторный аттрактор $\mathfrak{A}_{0}$ в топологии $\Theta_{+}^{\text {loc }}$, который не зависит от $N$, и справедливо неравенство

$$
\sup \left\{\|v(t)\|_{1}^{2} \mid t \geqslant 0\right\} \leqslant R^{2}, \quad \forall(u, v) \in \mathfrak{A}_{0} .
$$

Кроме того, $\mathfrak{A}_{0}=\Pi_{+} \mathscr{K}_{0}$, где $\mathscr{K}_{0}-$ ядро в $\mathscr{F}^{\mathrm{b}}$ системы $(4.15)-(4.16)$.

Сходимость траекторных аттракторов при $\delta \rightarrow \mathbf{0}+$. В работе [95] доказаны следующие основные результаты. 
ТеОрема 4.2. Траекторные аттракторы $\mathfrak{A}_{\delta}$ системы (4.1)-(4.2) сходятся при $\delta \rightarrow 0+$ в топологии $\Theta_{+}^{\text {loc }} \kappa$ траекторному аттрактору $\mathfrak{A}_{0}$ системиц (4.15)-(4.16):

$$
\mathfrak{A}_{\delta} \rightarrow \mathfrak{A}_{0} \quad(\delta \rightarrow 0+) \quad \text { в топологии } \Theta_{+}^{\text {loc }} .
$$

Заметим, что при любом $\theta \in] 0,1]$ топология $\Theta_{+}^{\text {loc }}$ сильнее топологии локальной сходимости в пространстве $C^{\operatorname{loc}}\left(\mathbb{R}_{+} ; H^{-\theta}(\Omega) \times H^{1-\theta}(\Omega)\right)$. Используя это свойство, получаем следующее утверждение.

СЛЕДСТВИЕ 4.2. При любом фиксированном $M>0$ и при любом $\theta \in(0,1]$ имеют место предельные соотношения

$$
\operatorname{dist}_{C\left([0, M] ; H^{-\theta}(\Omega) \times H^{1-\theta}(\Omega)\right)}\left(\mathfrak{A}_{\delta}, \mathfrak{A}_{0}\right) \rightarrow 0 \quad(\delta \rightarrow 0+) .
$$

Имея траекторные аттракторы $\mathfrak{A}_{\delta}$ и $\mathfrak{A}_{0}$ систем реакции-диффузии (4.1)-(4.2) и $(4.15),(4.16)$ соответственно, можно по схеме, изложенной в разделе 4 главы II, построить для этих систем слабые глобальные аттракторы

$$
\mathscr{A}_{\delta}:=\mathfrak{A}_{\delta}(0) \quad \text { и } \quad \mathscr{A}_{0}:=\mathfrak{A}_{0}(0) .
$$

При этом, очевидно, семейство глобальных аттракторов $\left\{\mathscr{A}_{\delta}, 0 \leqslant \delta \leqslant 1\right\}$ равномерно ограничено в пространстве $H^{0} \times H_{0}^{1}(\Omega)=H \times V$ и

$$
\mathscr{A}_{\delta} \rightarrow \mathscr{A}_{0} \text { слабо в } H \times V \text { и сильно в } H^{-\theta}(\Omega) \times H^{1-\theta}(\Omega) \text { при } \delta \rightarrow 0+.
$$

\section{Список литературы}

[1] А.В. Бабин, М.И. Вишик, Аттракторы эволюиионных уравнений, Наука, М., 1989; англ. пер.: A. V. Babin, M. I. Vishik, Attractors of evolution equations, Stud. Math. Appl., 25, North-Holland, Amsterdam, 1992.

[2] R. Temam, Infinite-dimensional dynamical systems in mechanics and physics, Appl. Math. Sci., 68, Springer-Verlag, New York, 1988.

[3] O.A. Ladyzhenskaya, Attractors for semigroups and evolution equations, Lezioni Lincee, Cambridge Univ. Press, Cambridge, New York, 1991, ISBN: 0-521-39030-3, xii+73 pp.

[4] J. K. Hale, Asymptotic behavior of dissipative systems, Math. Surveys Monogr., 25, Amer. Math. Soc., Providence, RI, 1988.

[5] И.Д. Чуешов, Введение в теорию бесконечномерных динамических систем, Акта, Харьков, 1999, ISBN: 966-7021-23-8, 436 с.; англ. пер.: I. D. Chueshov, Introduction to the theory of infinite-dimensional dissipative systems, University Lectures in Contemporary Mathematics, Acta, Kharkiv, 2002, 418 pp.

[6] G.R. Sell, Y. You, Dynamics of evolutionary equations, Appl. Math. Sci., 143, Springer-Verlag, New York, 2002.

[7] V. V. Chepyzhov, M. I. Vishik, "Trajectory attractors for evolution equations", C. $R$. Acad. Sci. Paris Sér. I Math., 321:10 (1995), 1309-1314.

[8] М. И. Вишик, В. В. Чепыжов, "Аттрактор неавтономной системы Навье-Стокса в трехмерном пространстве”, УМH, 50:4 (1995), 151; англ. пер.: V. V. Chepyzhov, M. I. Vishik, "An attractor of a non-autonomous Navier-Stokes system in threedimensional space", Russian Math. Surveys, 50:4 (1995), 807. 
[9] V. V. Chepyzhov, M. I. Vishik, "Trajectory attractors for 2D Navier-Stokes systems and some generalizations", Topol. Methods Nonlinear Anal., 8:2 (1996), 217-243.

[10] М. И. Вишик, В. В. Чепыжов, "Траекторные аттракторы эволюционных уравнений без однозначной разрешимости задачи Коши”, Вестн. МГУ. Сер. матем., мех., 110:6 (1996), 27-29; англ. пер.: M.I. Vishik, V. V. Chepyzhov, "Trajectory attractors of evolution equations without unique solvability of the Cauchy problem", Moscow Univ. Math. Bull., 51:6 (1997), 16-18.

[11] V. V. Chepyzhov, M. I. Vishik, "Evolution equations and their trajectory attractors", J. Math. Pures Appl. (9), 76:10 (1997), 913-964.

[12] V.V. Chepyzhov, M.I. Vishik, Attractors for equations of mathematical physics, Amer. Math. Soc. Colloq. Publ., 49, Amer. Math. Soc., Providence, RI, 2002.

[13] G. R. Sell, "Global attractors for the three-dimensional Navier-Stokes equations", J. Dynam. Differential Equations, 8:1 (1996), 1-33.

[14] О.А. Ладыженская, "О динамической системе, порождаемой уравнениями Навье-Стокса", Записки науч. сем. ЛОМИ, 27 (1972), 91-115; англ. пер.: О. А. Ladyzhenskaya, "A dynamical system generated by the Navier-Stokes equations", J. Sov. Math., 3:4 (1975), 458-479.

[15] О.А. Ладыженская, "О конечномерности ограниченных инвариантных множеств для системы Навье-Стокса и других диссипативных систем", Записки науч. сем. ЛОМИ, 115 (1982), 137-155; англ. пер.: О. A. Ladyzhenskaya, "Finite-dimensionality of bounded invariant sets for Navier-Stokes systems and other dissipative systems", J. Sov. Math., 28:5 (1985), 714-726.

[16] И. Д. Чуешов, "Глобальные аттракторы нелинейных задач математической физики", УМН, 48:3 (1993), 135-162; англ. пер.: I. D. Chueshov, "Global attractors for non-linear problems of mathematical physics", Russian Math. Surveys, 48:3 (1993), $133-161$.

[17] A. V. Babin, "Global attractors in PDE", Handbook of dynamical systems, 1B, Elsevier, Amsterdam, 2006, 983-1085.

[18] G. Raugel, "Global attractors in partial differential equations", Handbook of dynamical systems, 2, ed. B. Fiedler, North-Holland, Amsterdam, 2002, 885-982.

[19] A. Miranville, S. Zelik, "Attractors for dissipative partial differential equations in bounded and unbounded domains", Handbook of differential equations: evolutionary equations, 4, North-Holland, Amsterdam, 2008, 103-200.

[20] Ж.-Л. Лионс, Некоторые методы решения нелинейных краевых задач, Мир, М., 1972; пер. с фр.: J.-L. Lions, Quelques méthodes de résolution des problèmes aux limites non linéaires, Dunod, Graytier-Villars, Paris, 1969.

[21] J. Smoller, Shock waves and reaction-diffusion equations, Grundlehren Math. Wiss., 258, Springer-Verlag, New York-Berlin, 1983, ISBN: 0-387-90752-1, xxi+581 pp.

[22] В.К. Ванаг, Диссипативные структуры в реакционно-диффузионных системах, ИКИ, М.-Ижевск, 2008.

[23] J. C. Robinson, Infinite-dimensional dynamical systems, An introduction to dissipative parabolic PDEs and the theory of global attractors, Cambridge Texts Appl. Math., Cambridge Univ. Press, Cambridge, 2001.

[24] A. V. Babin, "Attractors of Navier-Stokes equations", Handbook of mathematical fluid dynamics, II, North-Holland, Amsterdam, 2003, 169-222.

[25] О.А. Ладыженская, Математические вопросы динамики вязкой несжимаемой жидкости, Физматгиз, М., 1961; 2-е изд.: Наука, М., 1970; англ. пер.: О. А. Ladyzhenskaya, The mathematical theory of viscous incompressible flow, Gordon and Breach, New York-London, 1963.

[26] Р. Темам, Уравнения Навъе-Стокса, Мир, М., 1981; пер. с англ.: R. Tеmam, Navier-Stokes equations, Stud. Math. Appl., 2, North-Holland, Amsterdam-New York-Oxford, 1979. 
[27] P. Constantin, C. Foias, Navier-Stokes equations, Chicago Lectures in Math., Chicago Univ. Press, Chicago, IL, 1988.

[28] A. Haraux, "Two remarks on dissipative hyperbolic problems", Nonlinear partial differential equations and their applications, Collège de France Seminar № 7, Research Notes in Math., 112, eds. H. Brezis, J.-L. Lions, Pitman, Boston, 1985, 161-179.

[29] J. M. Ghidaglia, R. Temam, "Attractors for damped nonlinear hyperbolic equations", J. Math. Pures Appl. (9), 66:3 (1987), 273-319.

[30] О.А. Ладыженская, "О нахождении минимальных глобальных аттракторов для уравнений Навье-Стокса и других уравнений с частными производными”, УМH, 42:6 (1987), 25-60; англ. пер.: O. А. Ladyzhenskaya, "On the determination of minimal global attractors for the Navier-Stokes and other partial differential equations", Russian Math. Surveys, 42:6 (1987), 27-73.

[31] J. Arrieta, A. N. Carvalho, J. K. Hale, "A damped hyperbolic equation with critical exponent", Comm. Partial Differential Equations, 17:5-6 (1992), 841-866.

[32] E. Feireisl, "Attractors for wave equations with nonlinear dissipation and critical exponent", C. R. Acad. Sci. Paris Sér. I Math., 315:5 (1992), 551-555.

[33] M. Grasselli, V. Pata, "On the damped semilinear wave equation with critical exponent", Dynamical systems and differential equations (Wilmington, NC, 2002), Discrete Contin. Dyn. Syst., suppl., 2003, 351-358.

[34] V. Pata, S. Zelik, "A remark on the weakly damped wave equation", Commun. Pure Appl. Anal., 5:3 (2006), 611-616.

[35] N. Kopell, L. N. Howard, "Plane wave solutions to reaction-diffusion equations", Stud. Appl. Math., 52 (1973), 291-328.

[36] Y. Kuramoto, T. Tsuzuki, "On the formation of dissipative structures in reactiondiffusion systems. Reduction perturbation approach", Progr. Theoret. Phys., 54:3 (1975), 687-699.

[37] A. Mielke, "The Ginzburg-Landau equation in its role as a modulation equation", Handbook of dynamical systems, 2, North-Holland, Amsterdam, 2002, 759-834.

[38] A. Mielke, "Bounds for the solutions of the complex Ginzburg-Landau equation in terms of the dispersion parameters", Phys. D, 117:1-4 (1998), 106-116.

[39] S. V. Zelik, "The attractor for a nonlinear reaction-diffusion system with a supercritical nonlinearity and its dimension", Rend. Accad. Naz. Sci. XL Mem. Mat. Appl. (5), 24 (2000), 1-25.

[40] C. R. Doering, J. D. Gibbon, C. D. Levermore, "Weak and strong solutions of the complex Ginzburg-Landau equation", Phys. D, 71 (1994), 285-318.

[41] J. M.Ghidaglia, B. Héron, "Dimension of the attractors associated to the Ginzburg-Landau partial differential equation", Phys. D, 28 (1987), 282-304.

[42] C. R. Doering, J. D. Gibbon, D. D. Holm, B. Nicolaenko, "Low-dimensional behaviour in the complex Ginzburg-Landau equation", Nonlinearity, 1:2 (1988), 279-309.

[43] A. Mielke, "The complex Ginzburg-Landau equation on large and unbounded domains: sharper bounds and attractors", Nonlinearity, 10 (1997), 199-222.

[44] М. И. Вишик, В.В. Чепыжов, "Неавтономное уравнение Гинзбурга-Ландау и его аттракторы”, Матем. сб., 196:6 (2005), 17-42; англ. пер.: M. I. Vishik, V. V. Chepyzhov, "Non-autonomous Ginzburg-Landau equation and its attractors", Sb. Math., 196:6 (2005), 791-815.

[45] V. V. Chepyzhov, M. I. Vishik, "Trajectory attractors for reaction-diffusion systems", Topol. Methods Nonlinear Anal., 7:1 (1996), 49-76; http://www-users.mat. uni.torun.pl/ tmna/files/v07n1-02.pdf.

[46] Ф. Хартман, Обыкновеннъе дифференциальные уравнения, Мир, М., 1970, 720 с.; пер. с англ.: Ph. Hartman, Ordinary differential equations, Wiley, New York-London-Sydney, 1964, xiv+612 pp. 
[47] Ж.-Л. Лионс, Э. Мадженес, Неоднородные граничные задачи и их приложения, I, Мир, М., 1971; пер. с фр.: J.-L. Lions, E. Magenes, Problèmes aux limites non homogènes et applications, 1, Dunod, Paris, 1968.

[48] П. С. Александров, А.Н. Колмогоров, Введение в теорию множеств и теорию функиий. Ч. 1. Введение в общую теорию множеств и функиий, Гостехиздат, M., 1948.

[49] J.-P. Aubin, "Un théorème de compacité", C. R. Acad. Sci. Paris, 256 (1963), $5042-5044$.

[50] Ю.А. Дубинский, "Слабая сходимость в нелинейных эллиптических и параболических уравнениях", Матем. сб., 67(109):4 (1965), 609-642.

[51] М. И. Вишик, В.В. Чепыжов, С.В. Зелик, "Сильный траекторный аттрактор диссипативной системы реакции-диффузии", Докл. РАН, 435:2 (2010), 155-159; англ. пер.: M. I. Vishik, V.V. Chepyzhov, S. V. Zelik, "Strong trajectory attractor for dissipative reaction-diffusion system", Dokl. Math., 82:3 (2010), 869-873.

[52] J. M. Ghidaglia, "A note on the strong convergence towards attractors of damped forced KdV equations", J. Differential Equations, 110:2 (1994), 356-359.

[53] I. Moise, R. Rosa, X. Wang, "Attractors for non-compact semigroups via energy equations", Nonlinearity, 11:5 (1998), 1369-1393.

[54] J. Valero, A. Kapustyan, "On the connectedness and asymptotic behaviour of solutions of reaction-diffusion systems", J. Math. Anal. Appl., 323:1 (2006), 614-633.

[55] V. Barcilon, P. Constantin, E. S. Titi, "Existence of solutions to the Stommel-Charney model of the Gulf stream", SIAM J. Math. Anal., 19:6 (1988), 1355-1364.

[56] J.-C. Saut, "Remarks on the damped stationary Euler equations", Differential Integral Equations, 3:5 (1990), 801-812.

[57] А.А. Ильин, "Уравнения Эйлера с диссипацией”, Матем. сб., 182:12 (1991), 1729-1739; англ. пер.: A. A. Il'in, "The Euler equations with dissipation", Math. USSR-Sb., 74:2 (1993), 475-486.

[58] Дж. Педлоски, Геофизическая гидродинамика, 1, Мир, М., 1984; пер. с англ.: J. Pedlosky, Geophysical fluid dynamics, Springer-Verlag, New York, 1979.

[59] В.И. Юдович, "Нестационарное течение идеальной несжимаемой жидкости", Ж. вычисл. матем. и матем. физ., 3 (1963), 1032-1066; англ. пер.: V. I. Yudovich, "Non stationary flow of an ideal incompressible liquid", USSR Comput. Math. Math. Phys., 3:6 (1963), 1407-1456.

[60] В. И. Юдович, "Некоторые оценки решений эллиптических уравнений", Матем. сб., 59(101) (дополнительный) (1962), 229-244.

[61] C. Bardos, "Existence et unicité de la solution de l'équation d'Euler en dimension deux", J. Math. Anal. Appl., 40:3 (1972), 769-790.

[62] V.V. Chepyzhov, M. I. Vishik, "Trajectory attractors for dissipative 2D Euler and Navier-Stokes equations", Russ. J. Math. Phys., 15:2 (2008), 156-170.

[63] V. V. Chepyzhov, M. I. Vishik, S. V. Zelik, "Strong trajectory attractors for dissipative Euler equations", J. Math. Pures Appl. (в печати).

[64] J. Leray, "Sur le mouvement d'un liquide visqueux emplissant l'espace", Acta Math., 63:1 (1934), 193-248.

[65] E. Hopf, "Über die Anfangswertaufgable für die hydrodynamischen Grundgleichungen", Math. Nachr., 4 (1951), 213-231.

[66] J. M. Ball, "Continuity properties and global attractors of generalized semiflows and the Navier-Stokes equations", J. Nonlinear Sci., 7:5 (1997), 475-502.

[67] А.Н. Колмогоров, И.Г. Петровский, Н. С. Пискунов, "Исследование уравнения диффузии, соединенной с возрастанием количества вещества, и его применение к одной биологической проблеме", Бюлл. Моск. гос. ун-та. Секиия А. Матем., мех., 1:6 (1937), 1-25. 
[68] A.I. Volpert, Vit. A. Volpert, Vl. A. Volpert, Traveling wave solutions of parabolic systems, Transl. Math. Monogr., 140, Amer. Math. Soc., Providence, RI, 1994, ISBN: 0-8218-4609-4, xii+448 pp.

[69] T. Ogiwara, H. Matano, "Stability analysis in order-preserving systems in the presence of symmetry", Proc. Roy. Soc. Edinburgh Sect. A, 129:2 (1999), 395-438.

[70] K. Kirchgässner, "Wave-solutions of reversible systems and applications", J. Differential Equations, 45:1 (1982), 113-127.

[71] A. Mielke, "Reduction of quasilinear elliptic equations in cylindrical domains with applications", Math. Methods Appl. Sci., 10:1 (1988), 51-66.

[72] Á. Calsina, X. Mora, J. Solà-Morales, "The dynamical approach to elliptic problems in cylindrical domains, and a study of their parabolic singular limit", J. Differential Equations, 102:2 (1993), 244-304.

[73] А.В. Бабин, “Аттрактор обобщенной полугруппы, порожденной эллиптическим уравнением в цилиндрической области", Изв. РАН. Сер. матем., 58:2 (1994), 3-18; англ. пер.: A. V. Babin, "The attractor of a generalized semigroup generated by an elliptic equation in a tube domain", Russian Acad. Sci. Izv. Math., 44:2 (1995), 207-223.

[74] B. Fiedler, A. Scheel, M. I. Vishik, "Large patterns of elliptic systems in infinite cylinders", J. Math. Pures Appl. (9), 77:9 (1998), 879-907.

[75] A. Scheel, "Existence of fast traveling waves for some parabolic equations: A dynamical systems approach", J. Dynam. Differential Equations, 8:4 (1996), 469-548.

[76] А. Мильке, С. Зелик, "Бесконечномерные траекторные аттракторы эллиптических краевых задач в цилиндрических областях", УМН, 57:4 (2002), 119-150; англ. пер.: A. Mielke, S. Zelik, "Infinite-dimensional trajectory attractors of elliptic boundary value problems in cylindrical domains", Russian Math. Surveys, 57:4 (2002), 753-784.

[77] М.И. Вишик, С. В. Зелик, “Траекторный аттрактор для нелинейной эллиптической системы в цилиндрической области", Матем. сб., 187:12 (1996), 21-56; англ. пер.: M. I. Vishik, S. V. Zelik, "The trajectory attractor of a non-linear elliptic system in a cylindrical domain", Sb. Math., 187:12 (1996), 1755-1789.

[78] М. И. Вишик, С. В. Зелик, "Регулярный аттрактор для нелинейной эллиптической системы в цилиндрической области", Матем. сб., 190:6 (1999), 23-58; англ. пер.: M. I. Vishik, S. V. Zelik, "Regular attractor for a non-linear elliptic system in a cylindrical domain", Sb. Math., 190:5-6 (1999), 803-834.

[79] С.В. Зелик, "Траекторный аттрактор нелинейной эллиптической системы в неограниченной области", Матем. заметки, 63:1 (1998), 135-138; англ. пер.: S. V. Zelik, "Trajectory attractor of a nonlinear elliptic system in an unbounded domain", Math. Notes, 63:1-2 (1998), 120-123.

[80] С. В. Зелик, "Ограниченность решений нелинейной эллиптической системы в цилиндрической области", Матем. заметки, 61:3 (1997), 447-450; англ. пер.: S. V. Zelik, "Boundedness of solutions of a nonlinear elliptic system in a cylindrical domain", Math. Notes, 61:3-4 (1997), 365-369.

[81] V. V. Chepyzhov, M. I. Vishik, "Perturbation of trajectory attractors for dissipative hyperbolic equations", The Maz'ya anniversary collection, Vol. 2 (Rostock, 1998), Oper. Theory Adv. Appl., 110, Birkhäuser, Basel, 1999, 33-54.

[82] S. Zelik, "Asymptotic regularity of solutions of singularly perturbed damped wave equations with supercritical nonlinearities", Discrete Contin. Dyn. Syst., 11:2-3 (2004), 351-392.

[83] М. И. Вишик, В. В. Чепыжов, "Усреднение траекторных аттракторов эволюционных уравнений с быстро осциллирующими коэффициентами”, Матем. сб., 192:1 (2001), 13-50; англ. пер.: M.I. Vishik, V. V. Chepyzhov, "Averaging of trajectory 
attractors of evolution equations with rapidly oscillating terms", Sb. Math., 192:1 (2001), 11-47.

[84] C. Cao, D. D. Holm, E. S. Titi, "On the Clark- $\alpha$ model of turbulence: global regularity and long-time dynamics", J. Turbul., 6 (2005), 1-11.

[85] S. Chen, C. Foias, D. D. Holm, E. Olson, E. S. Titi, S. Wynne, "The Camassa-Holm equations and turbulence", Phys. D, 133:1-4 (1999), 49-65.

[86] C. Foias, D. D. Holm, E. S. Titi, "The three dimensional viscous Camassa-Holm equations, and their relation to the Navier-Stokes equations and turbulence theory", J. Dynam. Differential Equations, 14:1 (2002), 1-35.

[87] S. Chen, C. Foias, D. D. Holm, E. Olson, E. S. Titi, S. Wynne, "A connection between the Camassa-Holm equations and turbulent flows in channels and pipes", Phys. Fluids, 11:8 (1999), 2343-2353.

[88] K. Mohseni, B. Kosović, S. Shkoller, J. E Marsden, "Numerical simulations of the Lagrangian averaged Navier-Stokes equations for homogeneous isotropic turbulence", Phys. Fluids, 15:2 (2003), 524-544.

[89] М. И. Вишик, Е. С. Тити, В. В. Чепыжов, "О сходимости траекторных аттракторов трехмерной $\alpha$-модели Навье-Стокса при $\alpha \rightarrow 0$ ", Матем. сб., 198:12 (2007), 3-36; англ. пер.: M. I. Vishik, E. S. Titi, V. V. Chepyzhov, "On convergence of trajectory attractors of the 3D Navier-Stokes $\alpha$-model as $\alpha$ approaches 0", Sb. Math., 198:12 (2007), 1703-1736.

[90] V. V. Chepyzhov, E. S. Titi, M.I. Vishik, "On the convergence of solutions of the Leray- $\alpha$ model to the trajectory attractor of the 3D Navier-Stokes system", Discrete Contin. Dyn. Syst., 17:3 (2007), 481-500.

[91] A. A. Ilyin, A. Miranville, E. S. Titi, "Small viscosity sharp estimates for the global attractor of the 2-D damped-driven Navier-Stokes equations", Commun. Math. Sci., 2:3 (2004), 403-426; http://projecteuclid.org/euclid.cms/1109868728.

[92] A. A. Ilyin, E.S. Titi, "Sharp estimates for the number of degrees of freedom for the damped-driven 2-D Navier-Stokes equations", J. Nonlinear Sci., 16:3 (2006), 233-253.

[93] R. FitzHugh, "Impulses and physiological states in theoretical models of nerve membranes", Biophys. J., 1 (1961), 445-446.

[94] J. Nagumo, S. Arimoto, S. Yoshizawa, "An active pulse transmission line simulating nerve axon", Proc. IRE, 50:10 (1962), 2061-2070; "Bistable transmission lines", Trans. IEEE on Circuit Theory, 12 (1965), 400-412.

[95] М.И. Вишик, В.В. Чепыжов, "О траекторных аттракторах систем реакциидиффузии с малой диффузией", Матем. сб., 200:4 (2009), 3-30; англ. пер.: M. I. Vishik, V. V. Chepyzhov, "Trajectory attractors of reaction-diffusion systems with small diffusion", Sb. Math., 200:4 (2009), 471-497.

[96] M. Marion, "Finite-dimensional attractors associated with partly dissipative reaction-diffusion systems", SIAM J. Math. Anal., 20:4 (1989), 816-844.

М. И. Вишик (M. I. Vishik)

Институт проблем передачи информации РАН

E-mail: vishik@iitp.ru

В. В. Чепыжов (V. V. Chepyzhov)

Институт проблем передачи информации РАН

E-mail: chep@iitp.ru
Поступила в редакцию 10.12.2010 